\title{
Preclinical studies on the immune-modulatory effects of dietary oligosaccharides
}

Citation for published version (APA):

Vos, A. (2008). Preclinical studies on the immune-modulatory effects of dietary oligosaccharides.

[Doctoral Thesis, Maastricht University]. Universiteit Maastricht. https://doi.org/10.26481/dis.20080529av

Document status and date:

Published: 01/01/2008

DOI:

10.26481/dis.20080529av

Document Version:

Publisher's PDF, also known as Version of record

\section{Please check the document version of this publication:}

- A submitted manuscript is the version of the article upon submission and before peer-review. There can be important differences between the submitted version and the official published version of record.

People interested in the research are advised to contact the author for the final version of the publication, or visit the DOI to the publisher's website.

- The final author version and the galley proof are versions of the publication after peer review.

- The final published version features the final layout of the paper including the volume, issue and page numbers.

Link to publication

\footnotetext{
General rights rights.

- You may freely distribute the URL identifying the publication in the public portal. please follow below link for the End User Agreement:

www.umlib.nl/taverne-license

Take down policy

If you believe that this document breaches copyright please contact us at:

repository@maastrichtuniversity.nl

providing details and we will investigate your claim.
}

Copyright and moral rights for the publications made accessible in the public portal are retained by the authors and/or other copyright owners and it is a condition of accessing publications that users recognise and abide by the legal requirements associated with these

- Users may download and print one copy of any publication from the public portal for the purpose of private study or research.

- You may not further distribute the material or use it for any profit-making activity or commercial gain

If the publication is distributed under the terms of Article $25 \mathrm{fa}$ of the Dutch Copyright Act, indicated by the "Taverne" license above, 


\section{Preclinical studies on the immune-modulatory effects of dietary oligosaccharides}

Arjan Paul Vos 
Preclinical studies on the immune-modulatory effects of dietary oligosaccharides. (C) 2008 Paul Vos

PhD thesis Maastricht University, the Netherlands.

ISBN/EAN: 9789064642425

The realization of this thesis was financially supported by Numico Research*, the Maastricht University, Friesland Foods Domo, de Jurriaanse Stichting, Beckman Coulter Nederland, Becton Dickinson Biosciences en Harlan Nederland.

Cover programming \& design: Paul Vos.

The cover shows a computer-generated laboratory scene, representing various aspects of the preclinical research described in this thesis. Depicted on the shiny, old-fashioned laboratory tiles are multiple short-chain galactooligosaccharides and one longer fructooligosaccharide molecule, relating to a specific mixture of oligosaccharides that was studied in this thesis. The pipette and ELISA plate were used frequently by the author to generate results, while the infant milk bottle represents an important practical application of the oligosaccharides and findings in this thesis.

Printed by Ponsen \& Looijen BV, Wageningen, The Netherlands.

*Will be called Danone Research - Centre for Specialised Nutrition as of 15 April, 2008.

All rights reserved to the author with the exception of those chapters published by the journals referred to. No part of this publication may be reproduced, stored in a retrieval system, or transmitted, in any form or by any means, without the prior permission in writing from the author or the copyright owning journal. 


\title{
Preclinical studies on the immune-modulatory effects of dietary oligosaccharides
}

\author{
Proefschrift \\ ter verkrijging van de graad van doctor aan de Universiteit Maastricht, \\ op gezag van de Rector Magnificus, prof. mr. G.P.M.F. Mols, \\ volgens het besluit van het College van Decanen, \\ in het openbaar te verdedigen op \\ donderdag 29 mei 2008 om 12.00 uur
}

door

Arjan Paul Vos 


\section{Promotores:}

Prof. dr. H. van Loveren

Prof. dr. J. Garssen (Universiteit Utrecht)

\section{Copromotor:}

Dr. ir. L. M'Rabet

\section{Beoordelingscommissie:}

Prof. dr. J.W. Cohen Tervaert (voorzitter)

Prof. dr. C.A. Bruggeman

Prof. dr. A.M.W.J. Schols

Prof. dr. Y. van Kooyk (Vrije Universiteit Amsterdam)

Dr. H. Verhagen (RIVM) 


\section{Contents}

$\begin{array}{lll}\text { Chapter } 1 \text { Preamble } & 7\end{array}$

Chapter 2 Immune-modulatory effects and potential working mechanisms of orally applied non-digestible carbohydrates; an introductory review

Chapter 3 A specific prebiotic oligosaccharide mixture stimulates delayedtype hypersensitivity in a murine influenza vaccination model

Chapter 4 Dietary supplementation of neutral and acidic oligosaccharides enhances Th1-dependent vaccination responses in mice

Chapter 5 Effects of a specific mixture of oligosaccharides in a murine vaccination model: supplementation around the priming phase enhances cellular immune responses that are correlated with cecal lactobacilli

Chapter 6 No evidence for immune-modulatory effects of specific neutral and acidic oligosaccharides in a limited set of in vitro assays

Chapter 7 Resistance to cytomegalovirus infection in mice is enhanced by a specific mixture of dietary oligosaccharides

Chapter 8 Dietary supplementation with specific oligosaccharide mixtures decreases parameters of allergic asthma in mice

Chapter 9 Specific oligosaccharides, low amounts of polyunsaturated fatty acids and a total concept of infant formula ingredients stimulate murine delayed-type hypersensitivity responses

Chapter 10 General discussion

Summary / Samenvatting

Dankwoord

Curriculum Vitae

List of publications

Author affiliations 



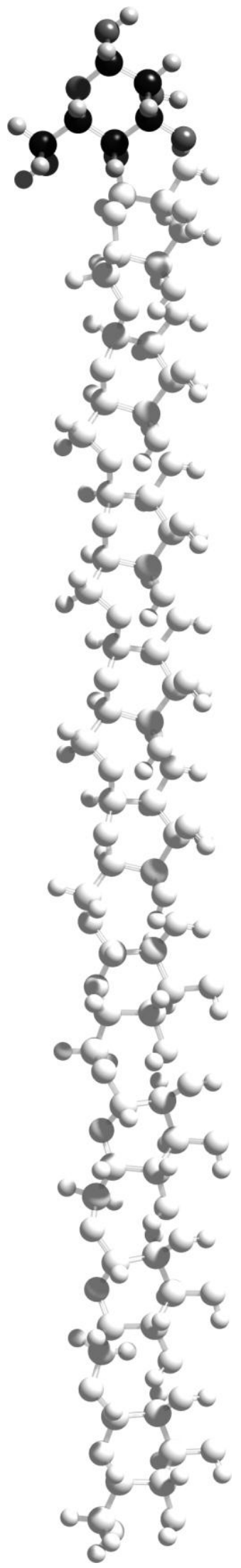

\section{Preamble}




\section{Background}

The health promoting and immune-modulatory effects of pre- and probiotic agents receive much attention nowadays, both in scientific literature and popular media. At the time of writing this thesis, an intensive advertising campaign is run on Dutch television that hints at the fact that $70-80 \%$ of the body's lymphoid tissue is located in or around the visceral organs (" $70 \%$ van je weerstand wordt in je buik bepaald, dat is waar je de voorsprong haalt"; advertisement for Danone Actimel) (1). Indeed, it is known that the intestinal microbiota have a large impact on immune function and development. This effect is most obvious in germ-free mice. Compared to conventional mice, germ-free mice display reduced numbers of systemic $\mathrm{T}$ and $\mathrm{B}$ cells, reductions in specific subsets of mucosal T cells, decreased cell-mediated immune responses, reduced immunoglobulin (Ig) A production, reductions in the number and size of Peyer's patches (PP), reduced size of mesenteric lymph nodes (MLN), and so on and so forth; conditions that can be normalized after conventionalization (reviewed in 2).

Human infants are devoid of bacteria before birth, but are colonized during delivery. Many factors influence this process and the subsequent development of the intestinal microbiota in the neonate. The mode of delivery is an important early determinant (3). After the initial colonization, the type of infant feeding plays an important role in the development of the microbiota. The microbiota in breast-fed infants quickly becomes dominated by bifidobacteria, whereas (standard) formula feeding leads to a relative increase in the number of Bacteroides and decreased amounts of bifidobacteria. In addition, a difference in the composition of the minor bacterial groups has been reported for both groups of infants (4). The 'bifidogenic' effect of breast milk is thought to be a multi-factorial phenomenon, in which the presence of a large amount of highly diverse oligosaccharides in human milk plays a major role (reviewed in 5).

Before the start of the experiments that are described in this thesis, an oligosaccharide concept was devised for application in infant formula, with the original goal to mimic the bifidogenic effects of human milk oligosaccharides (HMOS) (6). This concept consisted of short-chain galactooligosaccharides (scGOS) and long-chain fructooligosaccharides (scFOS) in a 9:1 ratio (Immunofortis; previously described in literature as GOS/FOS). Clinical studies demonstrated a bifidogenic effect of these oligosaccharides in pre-term and term infants, making the microbiota more 'breast-fedlike' $(7,8)$.

It has been hypothesized that the early development of the intestinal microbiota is 
related to allergic sensitization in the host (9). In addition, breast feeding has been associated with a reduction in atopic sensitization and infectious episodes in infants compared to (standard) formula feeding $(10,11)$. Combining these data, it was hypothesized that specific mixtures of oligosaccharides might induce immunemodulatory effects, possibly leading to an improved resistance to infections and/or a reduction in allergic sensitization or symptoms.

\section{Aim and outline of this thesis}

The aim of the work described in this thesis was to investigate the immune-modulatory effects of specific mixtures of oligosaccharides. These mixtures were designed for human food applications, primarily in infant formula. In order to gain insight into the immunological effects of these oligosaccharides, preclinical studies were performed in various experimental mouse models, including disease-specific models. Although the work was mainly focused on functional aspects of the immune-modulatory effects, attempts were made to unravel parts of the underlying mechanism as well, including a limited number of exploratory in vitro experiments.

The following main research questions were addressed in the experiments:

- Does dietary application of specific mixtures of oligosaccharides lead to modulation of systemic immunity?

- Is the immune-modulatory effect correlated to changes in the intestinal microbiota and is there evidence for microbiota-independent effects on the immune system?

- Are the observed effects consistent in multiple animal models, including diseasespecific models?

An introductory review of the literature on immune-modulating effects of dietary oligosaccharides and other non-digestible carbohydrates is presented in chapter 2 . It contains a comprehensive overview of the field including part of the data that is presented in the other chapters, as well as an exploration of the possible mechanisms underlying the immune-modulating effects.

Chapters 3 and 4 deal with the immune-modulatory effects of different oligosaccharides and oligosaccharide mixtures in a murine influenza vaccination model. The effects of the scGOS/lcFOS mixture are described in chapter 3, in comparison to other prebiotic agents. The interaction effects between scGOS/lcFOS and pectin-derived acidic oligosaccharides (pAOS) are described in chapter 4 . In both chapters, the effects of the oligosaccharides on the microbiota were explored as well. 
Aspects of the underlying working mechanism of oligosaccharide-induced immune modulation were studied in the vaccination model and in vitro. A detailed correlation analysis of the immunological and microbiota-related parameters in the vaccination model is presented in chapter 5 , in addition to an analysis of the phase of the immune response at which the modulatory effect appears to occur. Chapter 6 describes a limited selection of in vitro assays that were used to study potential microbiota-independent effects of the oligosaccharide mixtures.

To investigate whether the previously observed immune-modulatory effects translated into beneficial effects in specific disease conditions, the effects of the oligosaccharide mixtures were tested in a murine cytomegalovirus (MCMV) infection model (chapter 7) and a model of experimentally induced allergic asthma (chapter 8).

A novel infant formula concept was devised with the goal to support the infant's immune function and development optimally. It consisted of a mixture of oligosaccharides and multiple other potential immune-modulating ingredients, including n-3 and n-6 polyunsaturated fatty acids (PUFA). The immunological effects of the oligosaccharides in this concept were described in earlier chapters, but the net effect of the total concept is described in chapter 9. In addition, the effects of $n-3$ and $n-6$ PUFA-enriched diets were studied in a separate experiment that is described in chapter 9 as well.

Finally, the pattern of observed immunological effects in this thesis was compared to known immunological mechanisms in the literature. The findings are described in chapter 10, the general discussion, in addition to an account of the implications and the clinical relevance of the totality of findings.

\section{References}

1. Kagnoff, M.F., Immunology of the digestive system, in Physiology of the gastrointestinal tract, L.R. Johnson, Editor. 1987, Raven: New York. p. 1699-1728.

2. Smith, K., K.D. McCoy, and A.J. Macpherson, Use of axenic animals in studying the adaptation of mammals to their commensal intestinal microbiota. Semin Immunol, 2007. 19(2): p. 59-69.

3. Penders, J., C. Thijs, C. Vink, F.F. Stelma, B. Snijders, I. Kummeling, P.A. van den Brandt, and E.E. Stobberingh, Factors influencing the composition of the intestinal microbiota in early infancy. Pediatrics, 2006. 118(2): p. 511-21.

4. Harmsen, H.J., A.C. Wildeboer-Veloo, G.C. Raangs, A.A. Wagendorp, N. Klijn, J.G. Bindels, and G.W. Welling, Analysis of intestinal flora development in breast-fed and formula-fed infants by using molecular identification and detection methods. J Pediatr Gastroenterol Nutr, 2000. 30(1): p. 61-7.

5. Coppa, G.V., L. Zampini, T. Galeazzi, and O. Gabrielli, Prebiotics in human milk: a review. Dig Liver Dis, 2006. 38 Suppl 2: p. S291-4. 
6. Boehm, G., S. Fanaro, J. Jelinek, B. Stahl, and A. Marini, Prebiotic concept for infant nutrition. Acta Paediatr Suppl, 2003. 91(441): p. 64-7.

7. Boehm, G., M. Lidestri, P. Casetta, J. Jelinek, F. Negretti, B. Stahl, and A. Marini, Supplementation of a bovine milk formula with an oligosaccharide mixture increases counts of faecal bifidobacteria in preterm infants. Arch Dis Child Fetal Neonatal Ed, 2002. 86(3): p. F178-81.

8. Moro, G., I. Minoli, M. Mosca, S. Fanaro, J. Jelinek, B. Stahl, and G. Boehm, Dosage-related bifidogenic effects of galacto- and fructooligosaccharides in formula-fed term infants. J Pediatr Gastroenterol Nutr, 2002. 34(3): p. 291-5.

9. Kirjavainen, P.V. and G.R. Gibson, Healthy gut microflora and allergy: factors influencing development of the microbiota. Ann Med, 1999. 31(4): p. 288-92.

10. van Odijk, J., I. Kull, M.P. Borres, P. Brandtzaeg, U. Edberg, L.A. Hanson, A. Host, M. Kuitunen, S.F. Olsen, S. Skerfving, J. Sundell, and S. Wille, Breastfeeding and allergic disease: a multidisciplinary review of the literature (1966-2001) on the mode of early feeding in infancy and its impact on later atopic manifestations. Allergy, 2003. 58(9): p. 833-43.

11. Cushing, A.H., J.M. Samet, W.E. Lambert, B.J. Skipper, W.C. Hunt, S.A. Young, and L.C. McLaren, Breastfeeding reduces risk of respiratory illness in infants. Am J Epidemiol, 1998. 147(9): p. 863-70. 

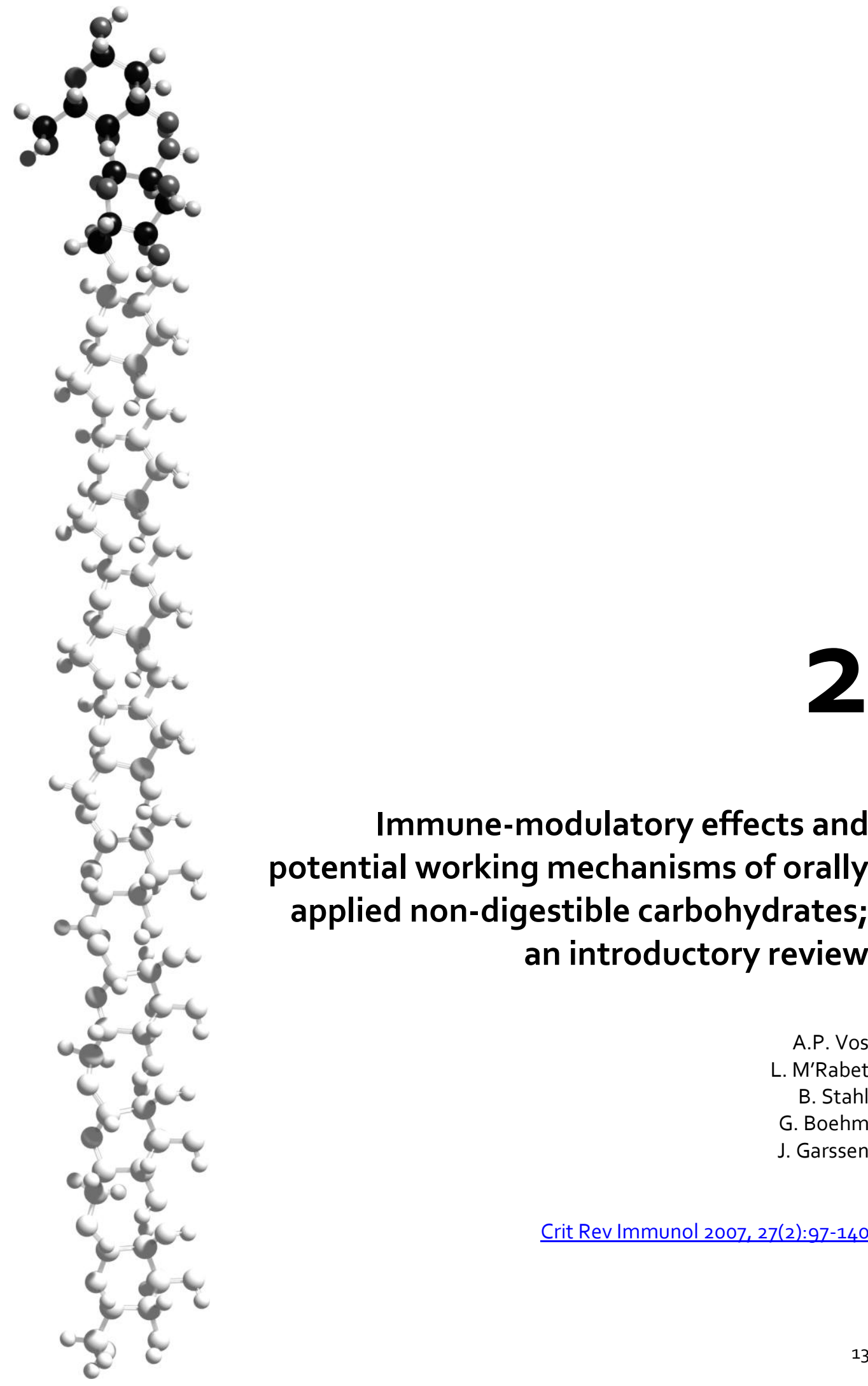

Immune-modulatory effects and potential working mechanisms of orally applied non-digestible carbohydrates; an introductory review

A.P. Vos

L. M'Rabet

B. Stahl

G. Boehm

J. Garssen 


\section{Abstract}

Orally applied non-digestible carbohydrates (NDC) have been associated with immune-modulating effects and other health benefits. The effects of prebiotic carbohydrates have recently received much attention, but other NDCs have been reported to induce immune modulation as well. Many different effects have been shown on parameters of innate and specific immunity, mostly in animal experiments or in vitro. Data from clinical trials are limited, but promising studies have reported beneficial effects on mucosal and systemic immunity in humans.

NDCs are fermented to various degrees by the intestinal microbiota. Therefore, immune-modulatory properties have often been attributed to microbiota-dependent effects, especially in the case of prebiotic NDCs. However, some NDCs have been reported to bind to specific receptors on cells of the immune system, suggesting microbiota-independent, immune-modulatory effects play a role as well.

This review aims to provide an overview of the published immune-modulatory effects in vitro and in vivo induced by NDCs such as fructans, galactooligosaccharides, $\beta$ glucans, pectins, and resistant starch. In addition, issues related to the underlying mechanisms are discussed: interaction between bacteria, their metabolites and the immune system, as well as direct effects of NDCs via lectin receptors.

\section{Table of contents:}

\section{Introduction}

2. Immune-modulatory effects

2.1. Human Milk Oligosaccharides

2.2. Fructans

2.2.1. In Vitro Experiments

2.2.2. Animal studies

2.2.2.1. Intestinal Inflammation

2.2.2.2. Systemic Inflammation and Infection

2.2.2.3. Tumor Formation and Growth

2.2.2.4. Local Intestinal Immune Modulation 
2.3.3. Galactooligosaccharides: Summarizing Remarks

2.4. Combinations of Galactooligosaccharides and Other Oligosaccharides

2.4.1. Animal Studies

2.4.2. Clinical Studies

2.4.3. Combinations of Galactooligosaccharides and Fructooligosaccharides: Summarizing Remarks

2.5.1. In Vitro Experiments

2.5.1.1. Modulation of Macrophage Functions and Immunological Responses

2.5.1.2. The Role of $\beta$-Glucan Receptors

2.5.2. Animal Models

2.5.3. Clinical Studies

2.5.4. $\beta$-Glucans: Summarizing Remarks

2.6.1. In Vitro Experiments

2.6.2. Animal Studies

2.6.3. Pectins: Summarizing Remarks

2.7. Resistant Starch

2.8. Other Nondigestible Carbohydrates

\section{Mechanism-related observations}

\subsection{Prebiotic Effects}

3.1.1. Prebiotic Effects of Oligosaccharides

3.1.2. Prebiotic Effects of Polysaccharides

3.2. Immune-Modulatory Mechanisms Related to Prebiotic Effects

3.2.1. Immune-Modulatory Effects of Probiotic or Commensal Bacteria

3.2.1.1. In Vitro Effects of Interaction Between Immune Cells and Probiotic Bacteria 49

3.2.1.2. Mechanisms of Interaction In Vivo $\quad 50$

$\begin{array}{ll}\text { 3.2.1.3. Animal Studies } & 51\end{array}$

3.2.1.4. Clinical Studies $\quad 52$

3.2.2. Immune-Modulatory Effects of Bacterial Metabolites $\quad 52$

3.3. Direct Interactions: Lectins 55

3.3.1. Brief Overview of Potential Interactions Between Lectins and Nondigestible Carbohydrates 55

$\begin{array}{lr}\text { 3.3.2. Lectins: Summarizing Remarks } & 56\end{array}$

\section{Conclusions}




\section{Introduction}

Non-digestible oligosaccharides and polysaccharides are natural constituents of many foods. They are often referred to as dietary fiber and are associated with many health benefits, including beneficial effects on fermentation, mineral absorption, barrier function, fat metabolism and cholesterol levels, glycemic and insulin responses, bile acid excretion, and the incidence of colon carcinoma (1).

The immune-modulating effects of these components are the topic of the current review, which is restricted to dietary carbohydrates that are not (fully) digestible by human or animal digestive tract endogenous enzymes. The emphasis throughout this review is on host immune modulation (i.e. modulation of cells or cell functions that play a role in immunological responses).

Plant-derived non-digestible carbohydrates (NDC) constitute the majority of dietary fiber in human food. The average daily intake of fiber in adults consuming Western diets is reported to vary from 16 to $44 \mathrm{~g} /$ day. The NDCs in food are very diverse in structure, including cellulose, pectins, inulins, gums, and resistant starch. Most NDCs are at least partially fermented in the large intestine. As a general rule, soluble NDCs such as inulin or pectin are well fermented, whereas insoluble NDCs such as cellulose or some forms of resistant starch are poorly fermented (2).

A major non-plant-derived source of NDCs is human breast milk. Non-digestible human milk oligosaccharides (HMOS) are normally present at approximately $10 \mathrm{~g} / \mathrm{L}$, making it the third major constituent in mature human milk (3). An overview of the main characteristics and food sources of the most studied NDCs in the context of immune modulation are shown in Table 1.

It was recognized long ago that the composition of the intestinal microbiota in breastfed infants was different from the composition of intestinal microbiota in early weaned children (4). It was recognized that carbohydrates cause this beneficial effect to the host (5). Nowadays, the HMOS-fraction is regarded as the first prebiotic agent that newborns encounter in life (6).

Prebiotics can be defined as "non-digestible food ingredients that beneficially affect the host by selectively stimulating the growth and/or activity of one or a limited number of bacteria in the colon" (7). Most NDCs are fermented at least partially in the large intestine, thereby affecting the growth and/or function of the local bacteria. According to the quoted definition, these carbohydrates qualify as prebiotics only if the observed stimulatory effects are specific for a (limited number of) bacterial species and if there is 


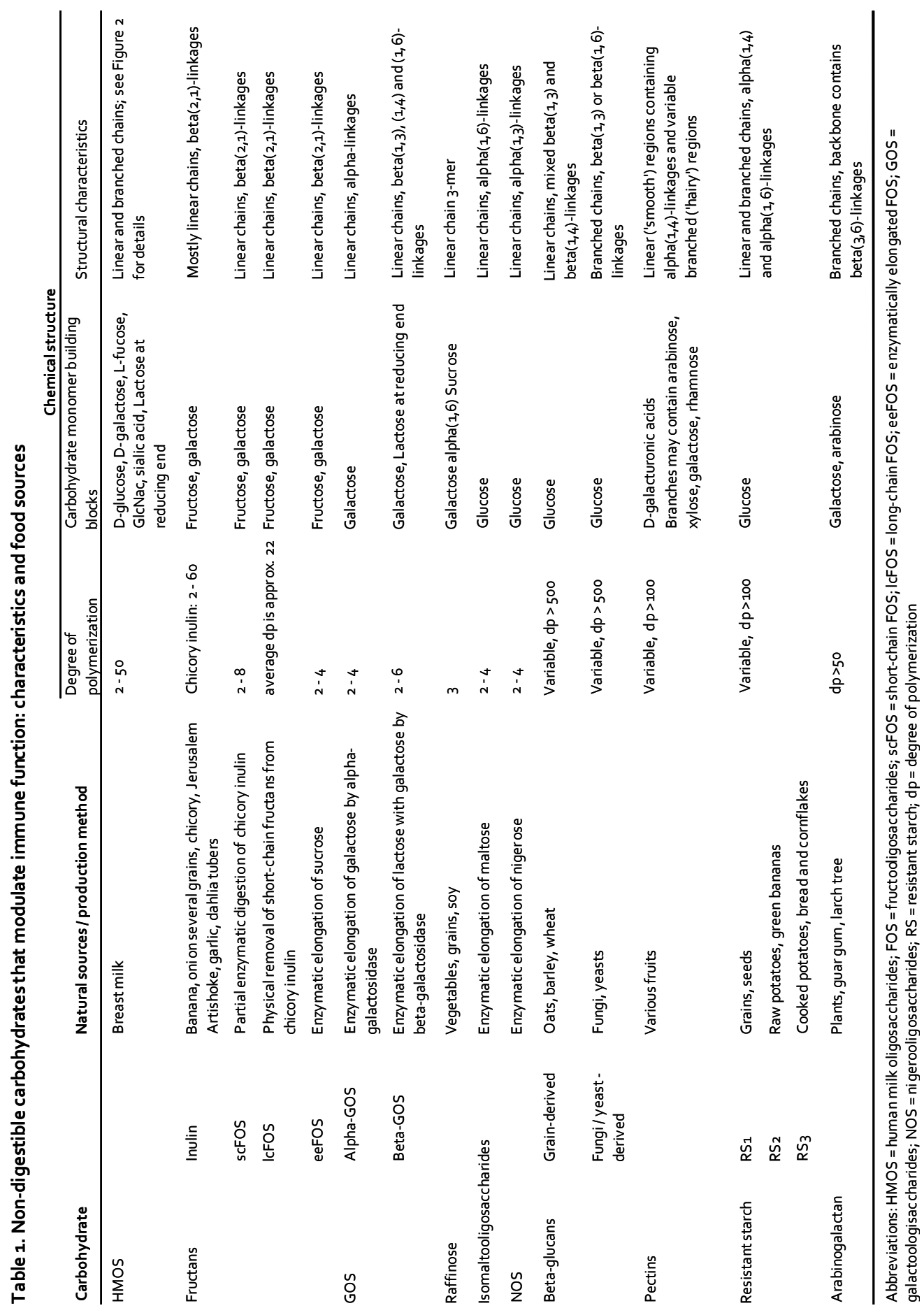


benefit for the host. Whether or not an NDC is a bona fide prebiotic agent according to this definition is not of major concern in this review; the term "prebiotic" is used freely throughout this article to indicate effects on microbiota, such as bifidobacteria and lactobacilli.

Bifidobacteria and lactobacilli belong to the group of lactic acid bacteria (LAB), which are regarded as beneficial when present in the gut. The growth and/or activity of these bacteria can be stimulated by application of prebiotic agents, but another approach is to administer such bacteria as orally applied probiotics. Probiotics can be defined as "live microorganisms administered in adequate amounts that confer a health effect on the host" (8). Because prebiotic and probiotic agents both affect the prevalence and/or activity of $\mathrm{LAB}$ in the intestine, immune-modulatory effects could be caused by microbiota-dependent mechanisms in both cases. However, prebiotic NDCs might also affect the host and/or the microbiota on the basis of their carbohydrate structure, for example, by activating or blocking cellular receptors, independent of the prebiotic effect. Potential mechanisms of NDC-induced immune modulation are discussed in this document; an overview is shown in Figure 1.

The literature on NDC-induced immune modulation is somewhat fragmented. The very active research field in the area of prebiotics focuses mainly on the effects of fructans and galactooligosaccharides (GOS). Immune-modulating effects of these carbohydrates are often studied under the assumption that the prebiotic effect is causative for the observed immune modulation. In contrast, $\beta$-glucans are widely studied immune-modulatory NDCs that are frequently assumed to induce direct receptor-mediated, immune-modulatory effects. The majority of publications dealing with $\beta$-glucans feature systemic application of $\beta$-glucans that are often derived from nonfood sources; specific $\beta$-glucans are even used in Japan for immune therapy in cancer patients $(9,10)$. However, $\beta$-glucans are also found in many food sources including various grains, edible mushrooms, and yeasts, but potential modulation of the microbiota and indirect effects on the immune system receive little attention in studies that focus on oral application of these components. Other groups of dietary NDCs including pectins, resistant starches, and others, have not been studied as elaborately in the field of immune modulation.

The aim of this review is to give a broad overview of the current evidence for immunemodulatory effects of orally applied NDCs in humans and experimental animals. Evidence for indirect, microbiota-dependent mechanisms, as well as direct, microbiotaindependent mechanisms is considered and discussed. To elaborate further on these possible working mechanisms, sections have been included that deal with immune modulation by commensal or probiotic bacteria, by their metabolites, and by 


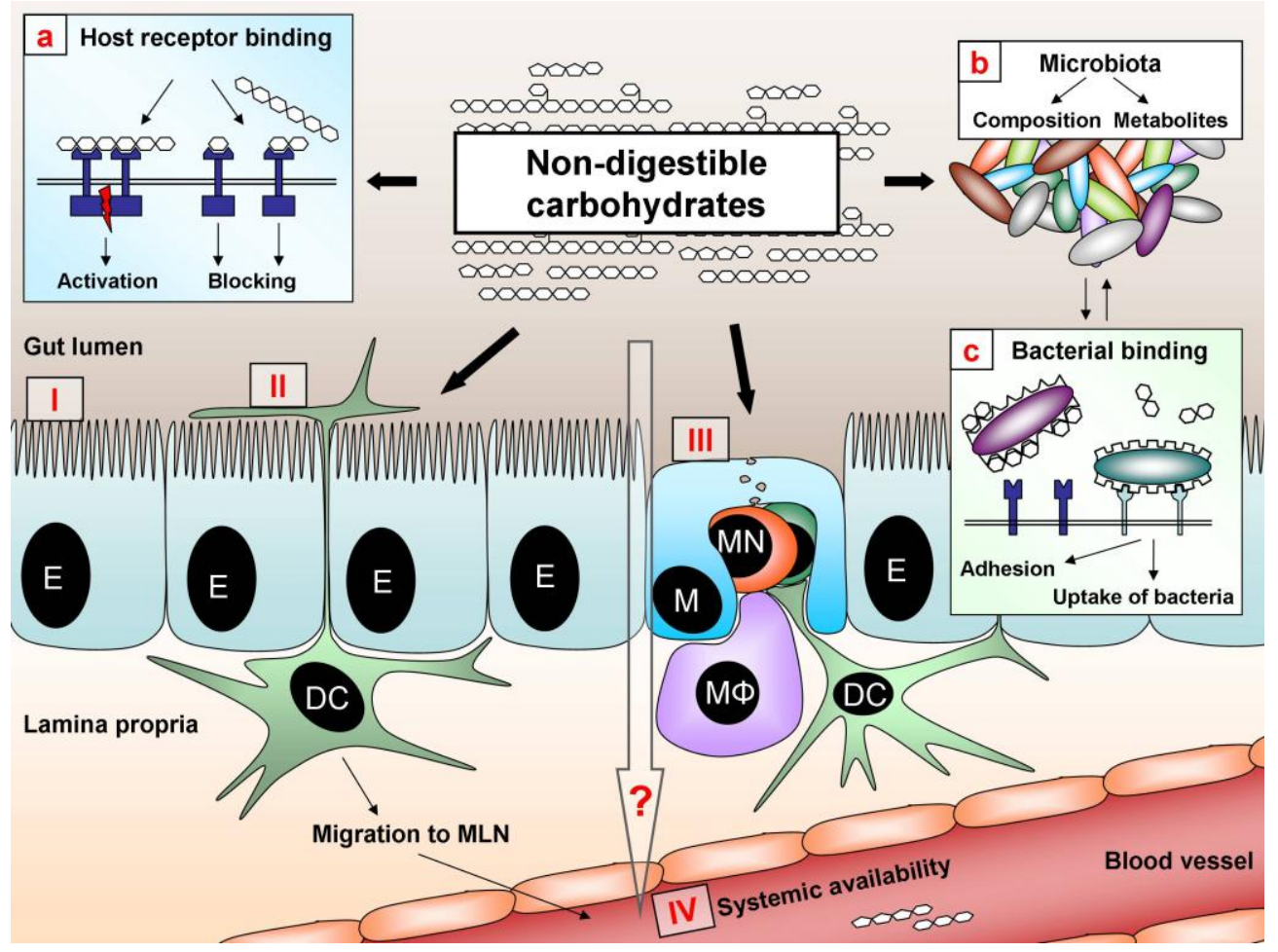

Figure 1. Potential mechanisms of immune modulation by orally applied non-digestible carbohydrates. Potential mechanisms of immune modulation by non-digestible carbohydrates (NDC) in the gastrointestinal tract are depicted schematically. Panel a: The mechanism of NDC-induced activation or blocking of host receptors that are involved in immunological responses by direct binding. Panel b: The mechanism of NDCinduced modulation of the composition of the microbiota and the production of bacterial metabolites. Panel c: The mechanism of direct interaction of NDCs with bacteria, by which adhesion to the mucosa and uptake of bacteria may be modulated. I: Intestinal epithelial cells (E) may play a role in receptor-mediated interaction with NDCs and bacterial adhesion that may be modulated by NDCs. II: Lamina propria dendritic cells (DC) have been described to sample the gut lumen. Receptor activation or blocking and modulation of bacterial uptake by NDCs may modulate the function of DCs. Because these DCs are know to migrate to mesenteric lymph nodes, mucosal and systemic immune-modulating effects might be induced. III: $M$ cells (M) are known to take up bacteria and soluble antigens from the gut. NDCs might modulate these mechanisms or be taken up by M cells, possibly affecting underlying immune cells including mononuclear cells (MN), macrophages (MФ), and DCs. IV: It has been described that dietary applied oligosaccharides are secreted in the urine in infants and adults. The uptake mechanism is unknown, but it may facilitate systemic immune-modulatory effects of NDCs.

carbohydrate-binding proteins called lectins. 


\section{Immune-modulatory effects}

\subsection{Human Milk Oligosaccharides}

HMOS are a mixture of carbohydrates with an enormous structural diversity (see Figure 2), containing a majority of short-chain neutral oligosaccharides that may be linear or branched, as well as smaller amounts of acidic sialylated oligosaccharides and long-chain oligosaccharides with lengths of up to 50 monomer units (11-13). A lactose moiety forms the reducing end, although this may be conjugated to other macromolecules in milk to form glycoproteins or glycolipids (14,15). Fractions of HMOS have been shown to act as decoy pathogen receptors: the host is passively protected by the HMOS, which bind to pathogen structures that are necessary for adhesion and infection. Targeted pathogens comprise bacteria, viruses, and fungi (14). It has been hypothesized that one function of this large variety of HMOS structures is to combat the large variety of pathogens that the infant may encounter. In accordance with this notion, the adhesion of various pathogens is inhibited by different fractions of HMOS (16). Additionally, HMOS may modify pathogen adhesion by modulation of intestinal glycosylation patterns, as an oligosaccharide that is present in human milk was shown to modify glycosylation patterns in a human colonic epithelial cell line (17).

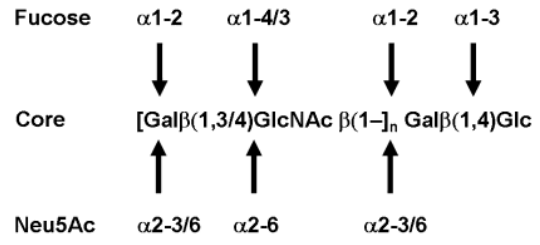

a

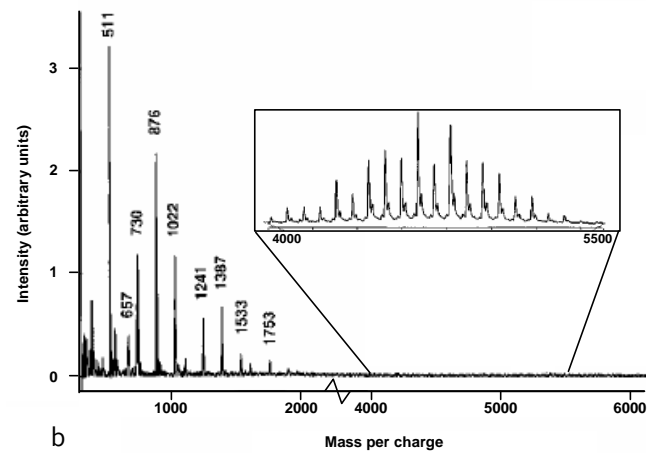

Figure 2. The basic structure and size distribution of human milk oligosaccharides. Human milk oligosaccharides (HMOS) are very diverse in structure and chain length. (a) The majority of HMOS feature a lactose unit (Gal- $\beta-(1,4)-G l c)$ at the reducing end and consist of the following building blocks: D-glucose (Glc), Dgalactose (Gal), $\mathrm{N}$-acetylglucosamine (GlcNac), L-fucose and sialic acid, or $\mathrm{N}$-acetylneuraminic acid (Neu5Ac). The arrows mark the possible glycosidic linkages to fucose or sialic acid residues. (Adapted from Boehm and Stahl (3).) (b) The size distribution of neutral HMOS was measured by MALDI mass spectrometry. HMOS consist of a majority of short chains and a minority of longer chain molecules. The mass peaks below a mass-to-charge ratio of 2000 represent oligosaccharides up to a degree of polymerization (dp) of 8 . The zoomed in section shows peaks representing oligosaccharides with longer chains ( $d p>20$ ). Adapted from Boehm et al (93). 
The concentration and variety of neutral HMOS varies largely between individuals and changes over the course of lactation (18). The expression pattern of HMOS was shown to be related to maternal secretor and Lewis blood group type $(19,20)$. Lewis blood group antigens are associated with helicobacter pylori infections (21), diarrhea incidence in infants (20), and other health effects. Further investigation is needed to investigate the potential role of HMOS in these matters.

HMOS may mediate systemic effects in infants, as they have been found in urine (22, 23). Systemic HMOS might affect pathogen adhesion at sites outside the gastrointestinal tract, and they might affect receptor binding and subsequent complex formation of immune cells as well, thereby affecting the immune response. In support of this idea, direct modulatory effects of HMOS on T cells in vitro have been found (24). In cultures with human cord blood cells, it was shown that acidic HMOS increased production of T-helper1 (Th1) and T-helper2 (Th2) cytokines and expression of CD25, a marker for activated or regulatory T cells. Possible target receptors for HMOS include selectins $(25,26)$, DC-SIGN (27), integrins $(28,29)$, galectins (30), and many other lectins.

There are indications that breast feeding modulates vaccination responses in infants compared with formula-fed children. Measles, mumps, and rubella vaccination induced significant changes in $\mathrm{CD}^{+} \mathrm{T}$ cells, natural killer (NK) cells, and mitogeninduced interferon (IFN)- $\gamma$ production compared with baseline in 1-year-old breast-fed children, but not in formula-fed children. No differences were observed in mitogeninduced interleukin (IL)-4 or IL-10 production, suggesting enhanced development of Th1 responses in breast-fed children (28). Another study demonstrated that breast feeding induced higher serum antibody responses to oral polio vaccine, but not to other systemic vaccines (31). In addition, multiple reports in the literature show that breast feeding lowers incidences of infections (32-34) and atopy-related disorders (35-37). However, it is not clear from these studies to what extent HMOS are responsible for these immunological effects compared with other factors in breast milk.

\subsection{Fructans}

Fructans are widely studied dietary carbohydrates that are used as prebiotic agents. Additionally, they have been described as agents that can modulate glucose and lipid metabolism $(38,39)$, and mineral absorption (40). Definitions of fructans, such as various types of fructooligosaccharides (FOS) and inulin, vary widely in literature. Most experiments that have been described in literature were performed with unprocessed chicory inulin or fructans derived thereof. Unprocessed chicory inulin is 
mainly composed of fructans with a degree of polymerization (dp) ranging from 2 to 60 , ending with a terminal glucose monomer. Throughout this article the term "inulin" refers to unprocessed chicory inulin unless stated otherwise. Partially hydrolyzed inulin has a typical dp range of 2 to 8 , and more molecules end without a terminal glucose monomer compared with inulin; it is referred to as short-chain FOS (scFOS) in this article. Physical removal of short-chain fructans from chicory inulin leads to a mixture of fructans with terminal glucose monomers and an approximate average dp of 22, referred to as long-chain FOS (lcFOS) in this article $(41,42)$. FOS can also be produced by enzymatic elongation of sucrose (eeFOS); it typically consists of very short fructan molecules of dp 2-4 (43). Illustrations of the basic molecular structures of fructans are shown in Figure 3.

\subsubsection{In Vitro Experiments}

Immune-modulating effects of fructans are mainly studied in vivo. However, in one report, inulin has been described to activate murine macrophage-like RAW264.7 cells in vitro. Endotoxin-free inulin induced nitric oxide and tumor necrosis factor (TNF)- $\alpha$ excretion when the cells were pretreated with IFN- $\gamma$. This effect was shown to be associated with activation of protein kinase $C-\alpha$ and the transcription factor nuclear factor- $\kappa B(N F-\kappa B)$. Given that the effects were measured in a culture system in vitro, no prebiotic mechanisms could have played a role in the observed effects. The working mechanism was not elucidated; it is therefore not known whether the observed effects are mechanistically related to the reported effects of $\gamma$-inulin (see section 2.2.4.) (44).

a

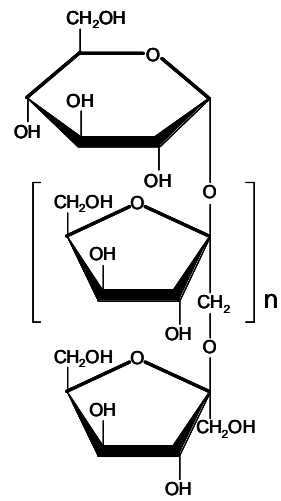

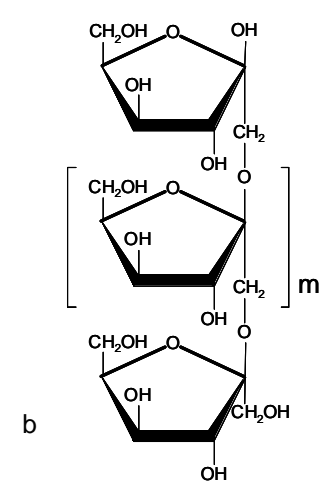

Figure 3. The basic structures of carbohydrate chains in fructans. Most fructose chains in fructan preparations end with a glucose residue at the reducing end (a). Chains that do not end with a glucose residue (b) are found in small amounts in unprocessed chicory inulin and in larger amounts in short-chain fructooligosaccharides (scFOS) derived from enzymatic digestion of inulin. Unprocessed chicory inulin mainly contains chains of $n=1-$ 59. scFOS preparations are mainly composed of very small chains $(n$ or $m<6)$ 


\subsubsection{Animal studies}

\subsubsection{Intestinal Inflammation}

Anti-inflammatory effects of fructans have been observed in several rat models for inflammatory bowel disease (IBD). In a trinitrobenzene sulfonate (TNBS)-induced colitis model, supplementation with an unspecified FOS preparation reduced disease scores and lowered several inflammatory cytokines and markers (45). Similar results were found in a transgenic spontaneous colitis model after supplementation with a combination of scFOS/lcFOS (Synergy 1). In addition, an up-regulation of transforming growth factor (TGF)- $\beta$ was found (46).

Mechanistic aspects of fructan-induced effects have been investigated in an experimentally induced colitis model. Oral application of an unspecified FOS preparation or inulin reduced disease scores in these experiments, parallel to increases in LAB and short-chain fatty acids (SCFA) in the colon. Oral application of LAB, as well as colonic application of LAB+SCFAs, resulted in similar reductions of disease symptoms. Colonic application of SCFAs resulted in partial reduction of symptoms compared with oral fructans, whereas colonic application of inulin did not have any effect $(47,48)$. These data indicate that fructans can ameliorate IBD symptoms in rat models by microbiota-dependent mechanisms. This fits well with the current idea that the microbiota play an important role in disease onset and perpetuation in IBD (49).

\subsubsection{Systemic Inflammation and Infection}

Apart from the anti-inflammatory effects observed in IBD-related animal models, stimulatory effects have been described on several immune parameters. In an endotoxemic shock model, $100 \mathrm{~g} / \mathrm{kg}$ dietary scFOS enhanced TNF- $\alpha$ and almost doubled prostaglandin E2 (PGE2) serum responses after a lipopolysaccharide (LPS) challenge. It increased the hepatic phagocytic Kupffer cell number and decreased serum alanine aminotransferase, a parameter for liver damage, suggesting altogether improved hepatic LPS clearance and reduced hepatoxicity (50). In apparent contrast, eeFOS supplementation of $7.5 \mathrm{~g} / \mathrm{L}$ in piglet milk resulted in decreased IL-6 induction 2 hours after ex vivo LPS stimulation of whole blood, but this effect was not measured at later time-points (51).

In systemic Listeria and Salmonella infection experiments in mice, high dietary doses $(100 \mathrm{~g} / \mathrm{kg})$ of scFOS or lcFOS increased survival compared with controls. Interestingly, lcFOS was more effective than scFOS and these effects were most striking in the Listeria model. Unfortunately, the effects of both fructans on the microbiota were not analyzed; the cause of the difference in effectivity remains unknown (52). 


\subsubsection{Tumor Formation and Growth}

In the same set of experiments in which effects on systemic infection were found, it was shown that scFOS and lcFOS inhibited carcinogen-induced formation of aberrant crypt foci. In addition, it was mentioned that these fructan supplemented diets enhanced NK activity, heightened "sensitivity" of lymphocytes and increased phagocytic activity of non-activated macrophages, but these data were not shown (52). Anti-carcinogenic effects have been reported for other fructans as well. In a spontaneous colon tumor model in transgenic mice, eeFOS reduced tumor incidence, whereas it enhanced the number of lymphoid nodules in the small intestine (53). In a follow-up experiment, it was shown that depletion of $\mathrm{T}$ cells increased the tumor incidence in supplemented mice, but the setup of the experiment did not allow for solid conclusions related to the underlying working mechanism (54). In contrast, low-dose inulin supplementation did not induce protective effects in the same transgenic tumor model (55). In a chemically induced rat tumor model, tumor-preventive effects were observed in mice treated with scFOS/lcFOS. Similar effects were observed in rats that received a synbiotic combination (combinations of probiotic bacteria and prebiotic substrates) of scFOS/lcFOS and probiotic bacteria, but probiotics alone were not as effective (56). In the prebiotic and synbiotic treated rats, production of IL-10 was elevated in Peyer's patch (PP) cell cultures, indicating a parallel effect of treatment on tumor- and immunerelated parameters (57).

Overall, there are multiple studies showing anti-carcinogenic effects of fructans in animal models for colon carcinoma. However, it is unclear whether these effects are mediated via immune modulation or other mechanisms, such as local effects of SCFAs on colonocytes. Beneficial effects have been reported in animal models of systemic tumors as well $(58,59)$. The working mechanisms were not elucidated, but in addition to the prebiotic effects, it was speculated that lipid or glucose metabolism-related mechanisms may have been involved as well.

\subsubsection{Local Intestinal Immune Modulation}

In one of the tumor models mentioned earlier, dietary supplementation with scFOS/lcFOS in healthy control rats resulted in enhanced IL-10 production in spleen cell cultures, enhanced spleen cell NK-activity, and a decreased ratio of $\mathrm{CD}^{+} / \mathrm{CD}^{+} \mathrm{T}$ cells (57). In another study from the same research group, scFOS/lcFOS was shown to enhance rat PP IL-10 production after mitogen stimulation and cecal secretory immunoglobulin A (sIgA) secretion. In addition, a combination of 2 probiotic strains was tested, as well as a synbiotic combination. The effects of the different treatments were not simply similar or additive: synbiotic treatment enhanced sIgA excretion in the 
ileum but not the cecum, whereas probiotics showed no effect at all. Probiotic and synbiotic treatment, in contrast to scFOS/lcFOS, did not enhance PP cytokine production (60).

Modulation of PP cytokine production has been observed in Balb/c mice as well. After 4 to 6 weeks of supplementation with eeFOS, Th1 and Th2 cytokines were produced at higher levels in PP cell cultures. PP IgA production was increased, in addition to elevated levels of IgA in the feces, although this latter effect appeared to be transient (61). Dietary eeFOS supplementation increased PP cellularity in healthy mice; the total cell number was increased, mainly due to an increase in B-cell numbers. In endotoxemic mice, both T- and B-cell counts were higher in PPs of supplemented animals than in controls (62). Supplementation of $5 \%$ eeFOS in infant mice, as well as their dams before weaning, increased PP IgA-positive, but not total or immunoglobulin M (IgM)-positive B cells. In addition, enhanced sIgA levels in gut and fecal samples, polymeric Ig receptor expression, and cecal acetate and butyrate concentrations were described (63).

\subsubsection{Clinical Studies}

Numerous clinical studies have been performed to investigate the effects of dietary fructan supplementation, but the number of studies that focused on immunemodulating effects is more limited.

Some beneficial effects have been observed in IBD patients. In patients with moderately active Crohn's disease, supplementation with a combination of scFOS/inulin (Prebio), up to $15 \mathrm{~g} /$ day, decreased disease scores. It tended to increase the IL-10 producing percentage of lamina propria dendritic cells (DC) from rectal biopsies as well, and increased their Toll-like receptor (TLR)2 and TLR4 expression. It should be noted that this was a very small open-label trial, so the data should be interpreted with caution (64). Supplementation of $24 \mathrm{~g} /$ day of inulin decreased histological inflammation scores in potential pouchitis patients without overt symptoms. This observation was associated with increased butyrate concentrations, a lowered $\mathrm{pH}$, decreased secondary bile acids, and decreased numbers of Bacteroides fragilis. Whether modulatory effects on the immune system caused the anti-inflammatory effect is not clear; changes in the gut content might have led to a decrease in disease-causing factors (65).

Synbiotic treatment of $12 \mathrm{~g} /$ day scFOS/lcFOS, combined with probiotics in colitis patients, resulted in a reduction of clinical inflammation symptoms, as well as a reduction in excreted $\beta$-defensins and inflammatory cytokines, which are both inflammatory markers. Because prebiotics were not tested without simultaneous probiotics in this study, it is unclear whether the observed effects are due to the 
prebiotics, the probiotics, or their simultaneous application (66).

Supplementation of healthy, free-living, elderly human subjects with $6 \mathrm{~g} / \mathrm{d}$ scFOS/inulin did not affect responses against pneumococcal and influenza vaccines, the number of infectious episodes in a half-year follow-up, or ex vivo cellular responses in comparison to the placebo group. The supplementation period prior to vaccination was only 1 or 2 weeks (this was not clearly described). If prebiotic effects would have been necessary for immune-modulatory effects to occur, this period might have been too short to induce modulation of the vaccination responses. Unfortunately, no effects of supplementation on the microbiota were analyzed; therefore, it is unknown whether and when prebiotic effects occurred in the study subjects (67).

Although not necessarily related to modulation of the host immune system, fructans have been tested for the prevention and treatment of diarrhea. Studies dealing with antibiotic-induced diarrhea are not within the focus of this review, but other studies may be relevant. A study on travelers' diarrhea showed some benefits of $10 \mathrm{~g} /$ day FOS (unspecified preparation) on self-reported well-being and some parameters of diarrhea scores. However, no significant effect on diarrhea prevalence was observed (68). In patients with Clostridium difficile-associated diarrhea, scFOS supplementation reduced the relapse rate compared with placebo, parallel to an increase in the number of bifidobacteria (69). It is unknown whether modulation of the host immune system played a role in the observed effects, or whether these were mediated solely by effects on the microbiota or direct effects on pathogen growth and/or adhesion.

It was reported that scFOS supplementation in combination with unphysiologically low calcium diets could negatively affect barrier function, bacterial translocation, and a diarrhea-associated parameter in rat studies (70-72). These data are in conflict with effects on diarrhea in other studies and prompted a double-blind, placebo-controlled crossover study in healthy volunteers. The effects of $20 \mathrm{~g}$ scFOS per day in combination with a well-controlled but calcium-restricted diet were not similar to the effects found in the animal models; no effect on fecal water cytotoxicity or permeability was observed, but mucin production was increased. Disputably, this was speculated to be indicative of irritation (73). However, 25-30 g/d scFOS did not negatively affect fecal water cytotoxicity or mucin excretion in a recent double-blind placebo-controlled study using healthy volunteers on a regular diet (74).

There are little data on fructan supplementation in infants with regard to immunemodulating effects. Supplementation with scFOS or scFOS+zinc did not enhance vaccination responses to Haemophilus influenzae and did not decrease the number of visits to clinics, the use of antibiotics or other indirect parameters related to immune function. However, many subjects in that study population received additional breast 
feeding along with solid foods, which could possibly explain the lack of prebioticrelated effect of scFOS (75). A scFOS-containing weaning food was reported to reduce symptoms of diarrhea, including fever episodes and medical consultation, but not diarrhea incidence. Additionally, cold-related symptoms and antibiotic use during cold episodes were reduced. All data were gathered by parental report (76). In a recent report, scFOS was studied in daycare-attending children. Although it was not the main focus of the study, significantly fewer fever episodes were reported in the scFOS groups compared with controls. A reduction in a combined parameter for intestinal discomfort and disease was also reported (77). Additionally, the enhancing effect of scFOS/inulin supplementation on measles vaccination-induced IgG titers in infants (7to 9-months old) was reported in an abstract, but the experimental data are not accessible (78).

Overall, there are indications that fructan supplementation increases parameters of immune competence in infants or young children, but more work is needed to substantiate these findings.

\subsubsection{Adjuvant Effects of $\gamma$-Inulin}

Interesting observations have been made decades ago regarding a specific insoluble form of inulin ( $\gamma$-inulin: a crystallized form of dahlia-tuber derived inulin of $\mathrm{dp}>50$ ) that activates the alternative complement pathway and shows adjuvant activity when injected intraperitoneally (79-81). Similar alternative complement activation effects have also been described for other insoluble glycans (82). In a series of experiments, the complement-activating activity of $\gamma$-inulin was shown to enhance the function of murine antigen-presenting cells (APC); it enhanced complement factor C3 production by macrophages as well as the stimulatory capacity of macrophages for antigen-specific $\mathrm{T}$ cells (83). Anti-tumor effects of intraperitoneally injected $\gamma$-inulin were observed, parallel to the complement activation mechanism both in dose and its dependence on insolubility. Soluble inulin showed no effects, or even opposite effects, when injected intraperitoneally (84). As stated before (see section 2.2.2), dietary application of fructans has been reported to reduce the incidence, growth, and metastatic capacity of tumors in animal models (not restricted to gut-related tumors), as well as being able to potentiate the cytotoxic effect of chemotherapeutic drugs $(58,59)$. Although these dietary effects are routinely attributed to prebiotic or metabolic effects of fructans, it is not clear from the current literature whether there is a common immune-modulating mechanism between $\gamma$-inulin and dietary fructan effects that does not involve the microbiota or its metabolites. 


\subsubsection{Fructans: Summarizing Remarks}

A wide range of effects has been reported from fructan supplementation in animal models, including anti-inflammatory effects in IBD-models, increased survival in infection models, anti-carcinogenic effects, enhanced mucosal antibody responses, and modulation of mucosal cytokine patterns and cell populations. Clinical studies have shown similar results in IBD patients, but other effects are not as clear. The data on infectious diarrhea and immune-modulating effects in infants and young children are interesting, but more data from well-controlled studies are needed to corroborate these findings.

Not many in vitro experiments have been performed with fructans. Because the prebiotic effects of fructans have been well established (see also section 3.1.1.), most experiments were carried out in vivo under the assumption that these microbiotadependent effects are causative of any immune-modulating effects that were measured. For example, multiple groups have reported effects of fructans on PP cells (57, 60-63). No attempts were made to correlate the observed immunological changes to changes in the microbiota in any of these studies. In addition, the levels of intestinal SCFAs were not measured in the majority of papers, making it impossible to draw conclusions related to the underlying working mechanisms. However, PPs are located in the small intestine, whereas fructans are fermented mainly in the large intestine. Therefore, it could be speculated that microbiota-independent mechanisms may play a role in the observed effects, but effects of low-level fermentation in the small intestine cannot be excluded. There is one study showing in vitro effects of inulin independent of effects on the microbiota (44); therefore, it is important that more research is done to confirm, or rule out, microbiota-independent effects. Additionally, because there are considerable differences in molecular structure among different fructan preparations, comparisons among different fructans are needed to gain insight into the structure-function relationship.

\subsection{Galactooligosaccharides}

Several types of GOS with different chemical characteristics have been used in research with a focus on immune-modulatory effects. $\beta$-Linked GOS produced by elongation of lactose using $\beta$-galactosidase enzymes is mostly referred to as trans-GOS or TOS in literature (see Figure 4 for an illustration of the basic molecular structure). In this review, it is referred to as $\beta$-GOS. $\alpha$-Linked GOS produced by elongation of galactose by $\alpha$-galactosidases is referred to as $\alpha$-GOS in this article $(85,86)$. 


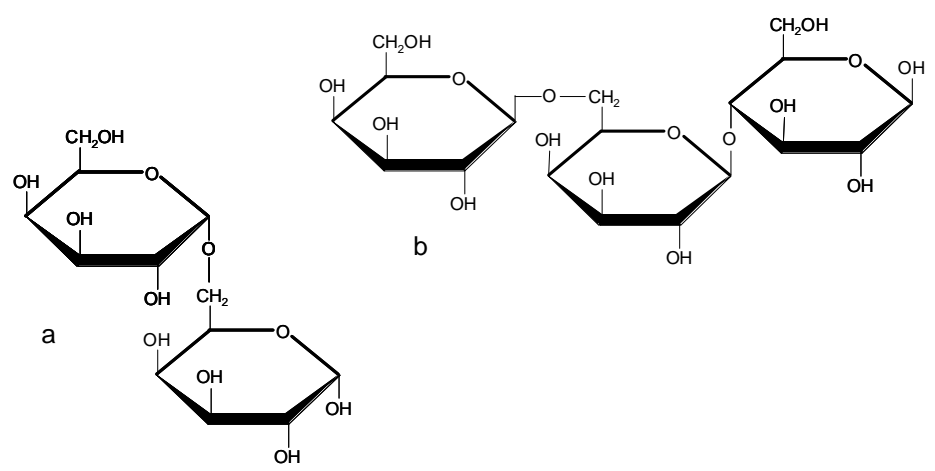

Figure 4. Examples of the chemical structure of $\alpha$ - and $\beta$-galactooligosaccharides (GOS). Enzymatic elongation of galactose or lactose is used to produce $\alpha$ - or $\beta$-GOS, respectively. A disaccharide with $\alpha-(1,6)$ linkage (a) is an abundantly produced component in the production of $\alpha-G O S$, but various other linkages and trisaccharides and tetrasaccharides occur as well (85). $\beta$-GOS may contain a variety of chain lengths (majority is $\mathrm{dp}<6)$ and linkages. An example is shown with a $\beta$ - $(1,6)$-linkage $(b)$.

$\alpha$-Galactosyl derivates of sucrose occur widely in nature; the trisaccharide raffinose and the tetrasaccharide stachyose are present in soy beans and many other plants. Although these oligosaccharides can be classified as $\alpha$-GOS, the trivial names are used throughout this article to avoid confusion with enzymatically produced $\alpha$ - and $\beta$-GOS.

\subsubsection{In Vitro Experiments}

It was recently reported that $\beta$-GOS inhibited adhesion of pathogenic E. coli to Hep-2 and Caco-2 epithelial cell lines more effectively than several fructans, raffinose, or lactulose (87). Inhibition of pathogen adhesion has also been described for HMOS (see section 2.1) and can be effective in prevention of infection. However, this effect is probably due to direct binding of NDCs to pathogens and thus does not involve modulation of the host immune system.

\subsubsection{Animal Studies}

$\beta$-GOS (4 g/kg/day) did not reduce inflammation in a TNBS-induced colitis model in rats, although it did increase fecal counts of bifidobacteria, as well as other bacterial counts on a nonspecific growth medium (88). It was speculated that TNBS-induced colitis is too severe to pick up beneficial effects of a prebiotic; however, such beneficial effects have been found after FOS supplementation (45).

Oral $\alpha$-GOS supplementation resulted in inhibition of lung eosinophilia and Th2related cytokine expression in a rat model for ovalbumin (OVA)-specific allergic 
asthma. In follow-up experiments, intra-peritoneal injection of $\alpha$-GOS yielded similar results, even when it was applied in animals that had undergone cecectomy and treatment with antibiotics to prevent any effect of treatment on the microbiota (89). These results strongly suggest that the immune-modulatory effects are not due to a prebiotic effect, but to a direct effect of $\alpha$-GOS on the immune system.

Immune-modulatory effects of orally applied $\alpha$-linked GOS were also shown in a collagen-induced arthritis model. Orally administered $\alpha$-GOS reduced clinical signs and nitrite/nitrate concentrations in serum, suggesting a down-regulation of inflammation. In apparent contrast, $\alpha$-GOS supplementation enhanced spontaneous and LPS-induced IL-1 $\alpha$ production by peritoneal macrophages ex vivo. Unfortunately, no further experiments were performed to unravel the underlying mechanism that caused the effects in vivo (90).

Dietary, as well as systemically applied raffinose, was shown to inhibit eosinophilia in the same allergic asthma model as mentioned for $\alpha$-GOS above. In addition, raffinose was shown to inhibit parameters of allergic inflammation $(89,91)$. Dietary raffinose increased IL-12 secretion in isolated PP cells of Balb/c mice. When used as APC, PP cells induced higher IFN- $\gamma$ in OVA-transgenic $\mathrm{T}$ cells when these were derived from raffinose-fed animals than from controls. Additionally, CD4+ T cells from mesenteric lymph nodes (MLN) from OVA-transgenic mice secreted less IL-4 when fed raffinose, which also decreased IgE levels in serum (92). Overall, raffinose appears to suppress Th2-related responses and stimulate Th1-related cytokine production.

\subsubsection{Galactooligosaccharides: Summarizing Remarks}

GOS has not been studied widely with respect to immune-modulating effects as a single dietary agent. Although anti-allergic and anti-inflammatory effects were described for $\alpha$-GOS and raffinose, more work is needed to study immune-modulating effects of GOS and compare these with immune-modulating effects of other oligosaccharides and NDCs. $\beta$-GOS has been studied more extensively in combination with lcFOS, which is described in section 2.4 .

\subsection{Combinations of Galactooligosaccharides and Other Oligosaccharides}

Combinations of $\beta$-GOS and other oligosaccharides have been used in research related to infant nutrition. A concept was devised (Immunofortis, previously referred to in literature as GOS/FOS) that aimed to mimic functional and general chemical properties of the neutral fraction of HMOS. It features a specific mixture of short-chain $\beta$-GOS and long-chain FOS in a 9:1 ratio (scGOS/lcFOS) (93), resembling the distribution of 
oligosaccharide chain lengths in human milk (see Figure $2 b)(11,12)$. This concept was recently extended by the addition of specific pectin-derived, partially unsaturated acidic oligosaccharides to mimic the acidic fraction of HMOS as described (94).

\subsubsection{Animal Studies}

In animal studies performed by the authors, the combination of scGOS/lcFOS was tested in an influenza vaccination model in C57BL/6 mice. A sub-optimal dose of trivalent influenza vaccine was used and emulsified with an oil-based adjuvant in order to have a sensitive model system to detect immune modulation. The animals were prefed with NDCs in their diets for 2 weeks before the first of 2 vaccinations. Low-dietary concentrations $(1 \%-2.5 \% \mathrm{w} / \mathrm{w}$ net oligosaccharides in the diets) enhanced systemic Th1-dependent, delayed-type hypersensitivity (DTH) responses after vaccination in several independent experiments and increased the proportions of fecal and colonic bifidobacteria and lactobacilli. No effects were observed on antigen-specific IgG antibody titers. Supplementation with similar doses of 3 different fructan preparations did not result in enhanced DTH responses. However, one of these increased the proportions of bifidobacteria and lactobacilli similar to the scGOS/lcFOS mixture. This suggests that a prebiotic effect alone is not sufficient for the immuneenhancing effect in this model (chapter 3).

The extended oligosaccharide concept was tested in the same vaccination model. The effects of the total combination of scGOS/lcFOS and the specific pectin-derived oligosaccharides on the DTH response were more pronounced than the separate components at comparable total dosages, indicating a form of synergy. The total combination induced prebiotic effects in fecal and gut samples as well. In contrast, supplementation with the acidic oligosaccharides alone enhanced the DTH response, without detectable changes in the relative amounts of bifidobacteria and lactobacilli in feces (chapter 4). Therefore, it is possible that the DTH-enhancing effect was (partially) microbiota-independent, similar to the effects of $\alpha$-GOS described above (89). Research into the mechanism of DTH and related contact hypersensitivity responses has shown that complement factor $\mathrm{C} 5 \mathrm{a}$ is involved in the initiation phase of these responses (95, 96). It could thus be speculated that effects on the complement system may have been involved in the observed enhanced DTH responses. However, the complementactivating effect that was described for $\gamma$-inulin and other glycans (see Section 2.2.4.) depends on a particulate, insoluble form. Because the tested NDCs were mainly soluble, it is not evident that this mechanism played a role in the observed effects.

In subsequent experiments the timing of NDC supplementation was varied. It was found that supplementation for 2 weeks prior to vaccination until 1 week afterwards 
resulted in a stimulation of the DTH response at the end of the experiment, similar to continuous supplementation. In contrast, when supplementation started at the day of the first vaccination until the end of the experiment (30 days in total), the DTH response was not significantly increased. This suggests that the modulation of the immune response occurred during the priming phase of the immune response. In addition, weak, but significant, correlations were found in control and supplemented groups between prebiotic and immunological parameters measured in individual mice (chapter 5).

Overall, these experiments are not conclusive with respect to the mechanism underlying the enhanced vaccine-specific DTH responses. Although microbiotaindependent effects can be speculated about, unmeasured or undetected effects on the microbiota could have played a role in all experiments. However, the experiments do suggest that more, or other, parameters than the proportions of bifidobacteria and lactobacilli play a role. More research is warranted in the area of prebiotic NDCs and immune modulation to further explore these issues.

\subsubsection{Clinical Studies}

Recently, the scGOS/lcFOS mixture was shown to reduce the incidence of atopic dermatitis in a population of infants at risk in a large double-blind, placebo-controlled trial. The mixture was administered in an infant formula; feeding started before 2 weeks of age in infants that did not receive any breast milk after 6 weeks of age. After 6 months, the cumulative incidence of atopic dermatitis was significantly improved (97). These results are in agreement with a recent report, in which it is suggested that the development of allergies is related to colonization with bifidobacteria and other microbiota (98).

In another clinical study, scGOS/lcFOS-supplemented formula increased fecal total sIgA compared with control formula. The sIgA excretion was measured every 4 weeks. A steady trend of increased sIgA concentrations was observed at all time-points and it was significant at 16 weeks of age. As expected, fecal sIgA levels in breast-fed subjects were much higher, especially very early in life due to the maternal antibodies in breast milk (99). In a small double-blind, placebo-controlled study, a "fermented infant formula" was used that was described to contain bifidogenic factors but not active bacteria. Dietary supplementation enhanced anti-polio fecal IgA responses after repeated vaccinations, and this effect was significantly correlated to an increase of specific bifidobacteria, relative to all cultivable flora $(100,101)$. The conclusions that can be drawn from this study are limited because of the small study population, as well as the lack of detail in the specification of the intervention. However, the results point in 
the same direction as the data from the previously mentioned paper (99), suggesting increased mucosal sIgA production after intervention with a bifidogenic factor.

\subsubsection{Combinations of Galactooligosaccharides and Fructooligosaccharides: Summarizing Remarks}

Multiple reports in literature have shown that the incidence of atopy-related disorders is lower in breast-fed infants than in those that are formula fed (35-37). It is remarkable that the addition of the scGOS/lcFOS mixture of oligosaccharides (Immunofortis) to infant formula has similar results in infants at risk for atopy (97). Atopy and allergy are related to Th2-immune responses, which are thought to inhibit Th1 responses and vice versa. Therefore, the Th1-stimulating effects found in animal studies fit well with the results of the clinical trial. More clinical trials are being planned and performed currently to further investigate the immune-modulating effects of these oligosaccharides on parameters that are relevant for allergies and infectious diseases.

The immune-modulating effects of scGOS/lcFOS in the clinical and animal studies are correlated with increases in bifidobacteria, similar to the effects of breast milk. Therefore, it is plausible that changes in the microbiota are causatively related to effects on the immune system. However, data from the animal studies suggest that other microbiota-related parameters or microbiota-independent effects might play a role as well. In addition, the results of the vaccination experiments indicate that various types of oligosaccharides have differential effects on the microbiota and the immune system.

\section{5. $\beta$-Glucans}

$\beta$-Glucans are polymeric glucose molecules linked together with $\beta$-glycosidic linkages. $\beta$-Glucans are present in many food sources, including various grains, edible mushrooms, and yeasts. Although yeast and fungi-derived $\beta$-glucans are branched or linear $\beta$-(1,3)-glucans or $\beta$-(1,6)-glucans (102-104), cereal $\beta$-glucans are mostly mixedlinkage linear $\beta$-(1,3)(1,4)-glucans (see Figure 5 for an illustration of the basic molecular structures) (105). The effects of $\beta$-glucans on lipid and carbohydrate metabolism have been studied widely in relation to cholesterol levels, diabetes, and weight management issues (106).

$\beta$-Glucans have been described as biological response modifiers as well: agents that modify the host's biological response by modulation of the immune system. Immunemodulating effects have been described in more than 1000 publications (107), dealing mostly with systemically applied $\beta$-glucans. Not all available data are reviewed here; the focus is on effects of oral and in vitro application of $\beta$-glucans from edible sources 


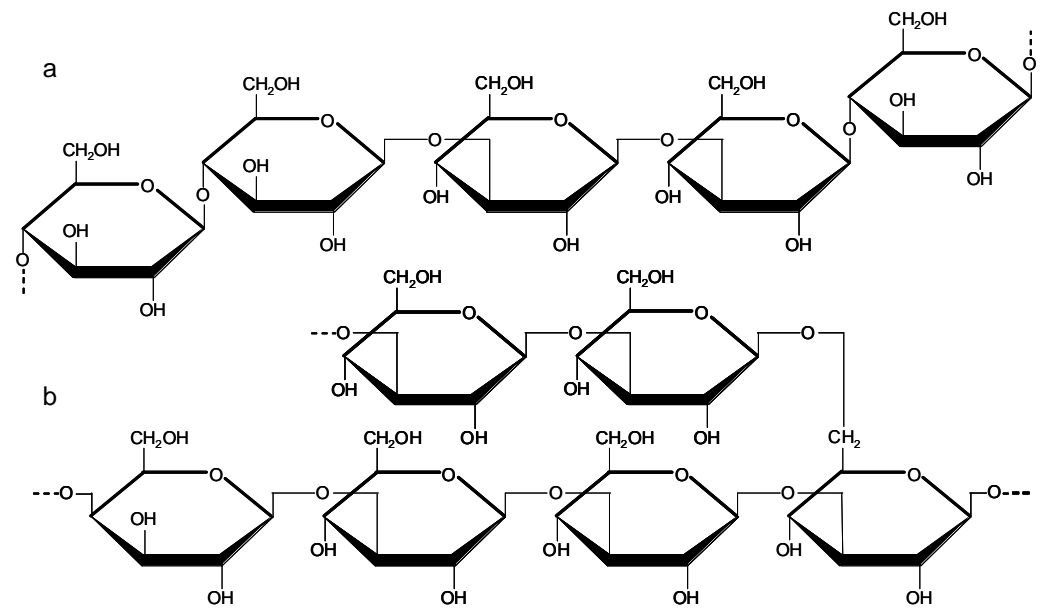

Figure 5. Examples of the chemical structure of $\beta$-glucans. B-glucans from grains such as oats or barley are mostly unbranched polysaccharides with semi-random alternating $\beta$ - $(1,3)$ and $(1,4)$-linkages (a). $\beta$-glucans from yeast and fungi are polysaccharides with a regular sugar backbone and a higher degree of branching. An example is shown with a $\beta-(1,3)$ backbone and a $\beta-(1,6)$ branch point $(b)$.

and on the role of $\beta$-glucan receptors.

The immune-modulatory effects of orally applied $\beta$-glucans have been reported in a wide variety of organisms such as shrimp $(108,109)$, fish $(110,111)$, poultry $(112)$, pigs $(113,114)$, mice $(115-118)$, and humans $(119,120)$. As these organisms belong to several unrelated biological taxa, these data might suggest the involvement of an evolutionary conserved pathway or mechanism leading to the observed immune modulation.

\subsubsection{In Vitro Experiments}

\subsubsection{Modulation of Macrophage Functions and Immunological Responses}

$\beta$-Glucans from various sources have been shown to modulate immune function in vitro. $\beta$-Glucan from Saccharomyces serevisiae, Baker's yeast, was reported to modulate inflammatory responses in human peripheral blood mononuclear cells (PBMC), or whole blood. An insoluble form of yeast $\beta$-glucan was shown to stimulate IL-1 receptor antagonist production in human PBMC and whole blood, which could be inhibited by soluble $\beta$-glucan (121). Additionally, insoluble yeast $\beta$-glucan increased expression of costimulatory molecules on murine macrophages (122).

It was shown recently that a soluble form of yeast $\beta$-glucan could increase IL-1 receptor 
antagonist production in parallel to increased IL-8 production. Interestingly, activation of NF- $\kappa \mathrm{B}$ and other transcription factors was shown, which did not lead to production of high levels of IL-1 $\beta$, IL-6, TNF- $\alpha$, or IFN- $\gamma$. LPS-induced TNF- $\alpha$ production was not affected either (123). In contrast, others reported that soluble $\beta$-glucan potentiated LPSinduced TNF- $\alpha$ and IL- 6 production, whereas $\beta$-glucan by itself stimulated IL- 8 production and low levels of IL-6 and IL-10 in human whole blood (124). In murine macrophage-like RAW264.7 cells, phagocytosis and TNF- $\alpha$ production were stimulated after in vitro incubation with soluble yeast $\beta$-glucan (125). Similar results were shown in murine peritoneal macrophages (126). In addition, it was shown that yeast $\beta$-glucans enhanced the production of inflammatory cytokines in IFN- $\gamma$-primed murine macrophages $(126,127)$.

Similar to yeast-derived $\beta$-glucan, $\beta$-glucans from edible mushrooms(128) and oats(129) have been reported to stimulate inflammatory cytokine production in murine macrophages or macrophage-like cells. In addition, production of T-cell-derived cytokines IL-2, IL-4, and IFN- $\gamma$ was stimulated in murine splenocytes (129). It was demonstrated that insoluble, but not soluble, barley $\beta$-glucan stimulated the alternative complement pathway (82). These data resemble the effects of inulin on complement activation; insoluble $\gamma$-inulin, but not soluble inulin, was shown to activate complement (81).

Overall, soluble and insoluble $\beta$-glucans from various sources have been shown to induce immune-modulatory effects in vitro, resulting generally in activation of macrophages and inflammatory responses. Different effects have been reported for soluble and insoluble forms of $\beta$-glucans, but also for different preparations with the same characteristics, making it impossible to form detailed hypotheses about structurefunction relationships on the basis of these data. Moreover, the $\beta$-glucan preparations were not always stringently tested for endotoxin contamination, devaluating the scientific value of the studies $(125,128)$.

\subsubsection{The Role of $\beta$-Glucan Receptors}

It has been suggested for many years that $\beta$-glucans induce immune-modulatory effects via specific receptors. Nowadays, multiple receptors have been described to bind $\beta$-glucans. IFN- $\gamma$ pretreatment of murine alveolar macrophages enhanced expression of $\beta$-glucan receptors, which was shown functionally by binding of a fluorescently labeled $\beta$-glucan to the cells. This priming effect by IFN- $\gamma$ was necessary for the activation of macrophages by the soluble $\beta$-glucans in these experiments, resulting in the production of inflammatory mediators (127). Inulin was found to induce similar effects on IFN- $\gamma$ treated macrophage-like RAW264.7 cells (44). This 
similarity is surprising because $\beta$-glucans and inulin differ in structure and are not known to share a common receptor.

Studies employing the human monocytic cell line U937 and its adherence to a glucan surface showed that a heptasaccharide was the smallest functional ligand for the $\beta$ glucan receptor on U937 cells. However, this heptasaccharide did not induce NF- $\kappa$ B activation or increase survival in a sepsis animal model, in contrast to high molecular weight $\beta$-glucan. These data suggest that a heptasaccharide is the smallest binding unit for the $\beta$-glucan receptor on these cells, but receptor activation occurs with large $\beta$ glucan molecules only, possibly indicating that receptor cross-linking is essential for activation. Unfortunately, the receptor was not characterized in this study (130).

The complement receptor CR3 has been implicated in $\beta$-glucan-mediated immunemodulating effects. A $\beta$-glucan-supplemented matrix enhanced polymorphonuclear leukocyte (PMN) motility in a CR3-dependent manner (131). Using human NK cells, $\beta$ glucan was shown to enhance NK activity in vitro in a CR3-dependent manner; blocking CR3 with a monoclonal antibody abrogated the observed effect $(132,133)$.

Recently, Dectin-1 has been identified as a major $\beta$-glucan receptor on macrophages (134). Human Dectin-1 is a C-type lectin that is expressed on many cells of the immune system including monocytes, macrophages, DCs, and granulocytes, as well as B cells and a subset of T cells (135). Dectin-1 can play an important role in cytokine production by DCs and has recently been shown to signal via 2 independent pathways in murine DCs. A Syk kinase-dependent Dectin-1 pathway was necessary for zymosan-induced IL-2 and IL-10 production, but a Dectin-1 and TLR2-dependent pathway could induce IL-12 production in Syk kinase knockout mice (136). In the current literature, many reports deal with $\beta$-glucan-induced modulation of macrophage functions. A few publications are available today on the effects of $\beta$-glucans on DCs $(137,138)$. As DCs play a central role in immunity - and it has been shown that DCs express Dectin-1 and many other pattern recognition receptors (PRR)-more research is needed to investigate the effects of $\beta$-glucans on DC function and maturation.

\subsubsection{Animal Models}

As mentioned, most immunological studies with $\beta$-glucans deal with systemic application of the agents. However, immune-modulatory effects of $\beta$-glucans have also been described after oral application. Yeast $\beta$-glucan was described to modulate several immune functions in pigs. Lung pathology in swine influenza virus-infected pigs was less severe in supplemented animals compared with controls, and viral replication in the lungs was reduced (114). In OVA-vaccinated pigs, oral yeast $\beta$-glucan enhanced antigen-specific humoral responses at one time-point, but not consistently during the 
experiment. In addition, LPS responses were measured in vitro and in vivo. In both cases, inflammatory cytokines were inhibited in the $\beta$-glucan supplemented group, whereas IL-10 production was increased (113). In mice, yeast $\beta$-glucan supplementation changed the intraepithelial T-cell populations in the gut. After 1 week of oral administration, the absolute number of $\alpha \beta$ and $\gamma \delta$ CD8+ T cells was increased. In liver lymphocytes, the orally applied $\beta$-glucans skewed spontaneous T-cell-derived cytokine production toward the Th1 type (117).

Intra-gastric oat $\beta$-glucan was reported to decrease fecal oocyst shedding in a murine Eimeria vermiformis infection model. Anti-parasitic humoral responses in serum and intestinal IgA responses were significantly increased as well, compared with controls $(118,139)$. Oral application of oat-derived $\beta$-glucan was shown to enhance viral resistance in peritoneal macrophages, both in normal and exercise stress-exposed mice (115).

Edible mushroom-derived $\beta$-glucan, lentinan, was reported to increase the number of peripheral T-helper cells in rats; however, the effect was only transient (140). In mice inoculated with human colon carcinoma cell lines, lentinan prefeeding for 1 week inhibited tumor growth (141). Oral barley $\beta$-glucans were shown to enhance anti-tumor effects of complement-activating monoclonal antibodies in systemic xenografted tumors in mice. The anti-tumor effect of intravenously injected CR3-specific antibodies was enhanced by dietary $\beta$-glucans, irrespective of the transplanted tumor type. Mixed $(1,3)(1,4)$-linked $\beta$-glucans from barley were more efficient than fungal $(1,3)(1,6)$-linked $\beta$-glucans $(142,143)$.

\subsubsection{Clinical Studies}

In a small placebo-controlled clinical study, 12 weeks of oral $\beta$-glucan supplementation resulted in modulation of cytokine levels in nasal lavage fluid after a provocation test in allergic subjects. In the supplementation group, Th2-related cytokines and the number of eosinophils in the lavage fluid decreased during the treatment period, whereas IL-12 production increased. In contrast, no significant changes were observed in the control group (120). In an early phase clinical trial with yeast $\beta$-glucan, no adverse effects were noted in subjects receiving oral supplementation of up to 400 $\mathrm{mg} /$ day, and no systemic uptake of $\beta$-glucans was measured in serum, but IgA was increased in saliva (119).

\subsection{4. $\beta$-Glucans: Summarizing Remarks}

$\beta$-Glucans are a diverse group of NDCs that induce a wide variety of immunological effects. In vitro experiments clearly show that $\beta$-glucans have direct, receptor-mediated 
effects on various cells of the immune system, most notably on macrophages. However, the effects are not all consistent; for example, macrophages have been described to produce anti-inflammatory IL-1 receptor antagonist production after $\beta$-glucan exposure $(121,123)$, as well as inflammatory cytokines such as TNF- $\alpha$ and IL-6 (124-126). In pigs, the production of the regulatory cytokine IL-10 was enhanced whereas inflammatory cytokines were decreased (113). It is not clear from the literature whether these differences are due to differences among different $\beta$-glucan preparations or to variation among experimental models and laboratories.

Immune-stimulating effects have been described, including increased resistance to various infections and anti-tumor effects. Animal models have shown systemic immune-modulatory effects, even though $\beta$-glucans are not expected to cross the intestinal barrier because of their molecular size, unless specific uptake takes place. Indeed, $\beta$-glucans were not detected in serum of supplemented individuals in a clinical study (119). However, several animal studies have shown systemic effects, including interaction of $\beta$-glucans with systemically applied antibodies and effects on viral parameters in the lungs $(114,142,143)$. Although multiple receptors for $\beta$-glucans have been identified on various immune cells, it is currently unknown how orally applied $\beta$ glucans modulate systemic immune parameters. The potential role of $\beta$-glucan fermentation, including the effects on the microbiota and their metabolites (see section 3.1.2.), is not currently addressed in the literature with regard to immune-modulatory effects.

\subsection{Pectin}

Pectins are components of plant cell walls that are abundant in various fruits and consist mainly of long, linear $(1,4)$-linked polymers of D-galacturonic acid, as illustrated in Figure 6. Additionally, cell-wall pectins consist of complex branched regions of highly variable composition and order of monosaccharide units. Pectins are capable of forming gels and holding water and are fermented up to $90 \%$ in the colon (144). Pectin molecules are cleaved into smaller fragments during in vivo fermentation and food processing, as well as by enzymatic hydrolysis and $\mathrm{pH}$ modification to create acidic pectin-derived oligosaccharides with beneficial properties $(145,146)$. 


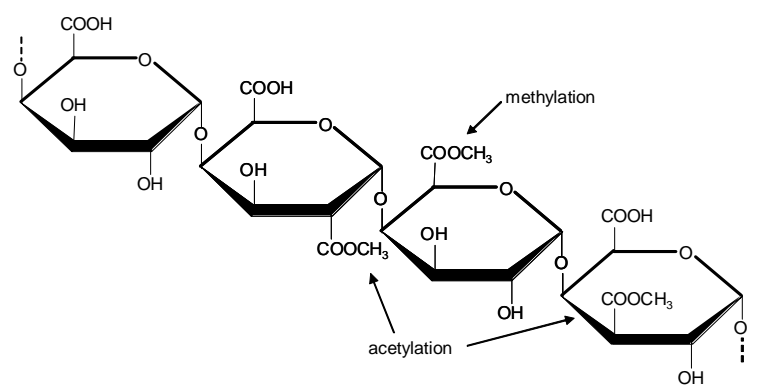

Figure 6. The basic chemical structure of pectins. Pectins are heterogeneous molecules consisting of "smooth" linear and "hairy" branched regions that are highly variable (not depicted). The linear regions consist of $\alpha-(1,4)$-linked galacturonic acid residues that may be methylated or acetylated to various degrees. Pectins show various degrees of branching and may contain various other carbohydrate residues.

\subsubsection{In Vitro Experiments}

A few studies have been published regarding immunological effects in vitro induced by pectins or pectic substances. It was reported that a pectic substance from a Brazilian fruit tree activated murine peritoneal macrophages. However, this effect was measured only morphologically; no cytokines or other mediators were measured (147). Pectic polysaccharides from almond cell-wall material activated B cells and, to a lesser extent, T cells, as measured by flow cytometric analysis of the activation markers CD25 and CD69 after 3 days in vitro incubation. Macrophage cytokine production was not affected (148). Citrus pectins with varying degrees of esterification inhibited LPSinduced activation of murine macrophage-like RAW264.7 cells. The inhibition was positively correlated to the degree of esterification. In addition, it was shown that the most effective pectin was able to bind directly to LPS. Therefore, the observed effects could have been related to the inactivation of LPS by pectin, instead of antiinflammatory effects on the macrophage-like cells (149).

Pectin-derived acidic oligosaccharides from carrots were described to inhibit pathogen adherence to uro-epithelial cells and the human intestinal mucosa in vitro (150, 151). Pectin and pectic oligosaccharides were shown to neutralize the activity of Shiga-like toxins from Escherichia coli, thereby increasing cell survival in a culture of human colonic cells. Short pectic oligosaccharides were found to be more effective than longer molecules in these experiments $(150,152)$. These results suggest that pectic oligosaccharides may passively protect the host in vivo by inhibiting pathogen adherence and toxin activity. 


\subsubsection{Animal Studies}

Asian pear pectin and an unspecified commercial pectin preparation were studied in an OVA-specific allergy model in Balb/c mice. The animals received oral doses of pectin or saline daily for 1 week before sensitization. After sensitization and subsequent OVAprovocation, both pectin treatments decreased airway smooth-muscle sensitivity and microscopically scored lung tissue damage. Different effects of pectin treatment were observed on IFN- $\gamma$ and IL-5 levels in bronchoalveolar lavage and phorbol myristate acetate/ionomycin stimulated splenocytes: a reduction in IFN- $\gamma$ and stimulation of IL-5 was found in the lavage fluid, whereas the opposite effects were observed in supernatants from stimulated splenocytes. Unfortunately, not all data were shown and the quality of data analysis was unclear in this publication (153).

As a replacement for the standard source of dietary fiber, an unspecified pectin preparation induced effects on MLN cell antibody production and CD4 $4^{+} \mathrm{CD}^{+}$ratios of $\mathrm{T}$ cells. In pooled MLN cultures, spontaneous and Concanavalin A (ConA)-induced IgA production appeared to be stimulated and IgE production reduced. In serum, total titers of IgG and IgA were significantly increased, whereas IgE was significantly decreased. In spleens, the $\mathrm{CD}^{+} / \mathrm{CD}^{+} \mathrm{T}$-cell ratio was significantly increased (154). In a similar experiment using a dextran sodium sulfate (DSS)-induced colitis model, fecal IgA secretion was stimulated highly in pectin-treated mice. Additionally, MLN-derived ConA-stimulated lymphocytes produced significantly more Th1 cytokines, but less TNF- $\alpha$ in DSS-treated mice. Th2 cytokines were decreased in DSS-treated mice but unaffected in DSS-untreated mice (155).

A number of animal studies have been carried out with modified citrus pectin ( $\mathrm{pH}-$ modified citrus pectin to produce shorter pectin fragments) in the field of oncology. Binding to galectin-3 was suggested. Galectin-3 is a lectin molecule that plays a role in cell adhesion and the formation of metastases. Application of modified citrus pectin in the drinking water inhibited metastasis formation and tumor growth in several systemic rat tumor models; it was suggested that binding to galectin-3 played a role in the observed effects (156-158). A small-scale, nonrandomized clinical study in prostate cancer patients yielded results similar to the animal studies, suggesting a reduction in tumor growth (159). It is unclear from these studies whether modulation of immune responses played a role in the observed effects; however, the results do suggest that modified citrus pectin is able to induce systemic effects.

Specific pectin-derived, partially unsaturated acidic oligosaccharides induced immunemodulatory effects in our own experiments; we used the murine vaccination model that was described earlier (see section 2.4.1.). Supplementation of $1 \%$ to $5 \%(\mathrm{w} / \mathrm{w})$ in the 
diet dose dependently enhanced vaccine-specific DTH responses, a Th1-related parameter. In ex vivo splenocyte cultures stimulated with the vaccine antigens, Th2related cytokine production (IL-4, IL-5, and IL-10) was reduced in a dose-dependent fashion, whereas production of IL- 2 and IFN- $\gamma$ remained constant. These data suggest a shift toward Th1-related immunity in a systemic vaccination response. In a follow-up experiment, it was found that supplementation of $2 \%$ did not affect the proportion of bifidobacteria and lactobacilli in fecal samples, whereas the DTH-response was enhanced significantly (chapter 4, 160). These results suggest that prebiotic effects are not necessary for modulation of systemic immune responses by dietary application of these specific NDCs. However, other, or low-level effects on the microbiota cannot be excluded.

Immune-modulatory effects of orally applied pectic substances from herbal or other non-food plants have been shown in the literature as well; for example, increased antibody responses and B-cell proliferation $(161,162)$, complement fixing, mitogen and chemoattractant activity $(163,164)$, oral adjuvant activity (165), increased DTH responses (166), increased phagocytosis, and oxygen radical production(166) were described.

\subsubsection{Pectins: Summarizing Remarks}

The available data on the immune-modulating effects of orally applied pectins are limited mostly to animal experiments. Pectins are highly variable polysaccharides and it was shown that different degrees of methylation and different chain lengths induce different biological effects.

Several publications have reported results of pectin or pectin-derivates that indicate Th2 $\rightarrow$ Th1-skewing effects. The working mechanism underlying these immunemodulating responses was not clarified, and the effects need to be confirmed in the human situation. A galectin-3-dependent mechanism was suggested to play a role in the observed anti-tumor effects of modified citrus pectin. It remains to be established whether modulation of immune responses occurs as well.

\subsection{Resistant Starch}

Starch that is not digested in the small intestine and enters the hindgut is defined as resistant starch (RS). Three main types of resistant starch have been identified: $\mathrm{RS}_{1}$ is physically inaccessible starch that is present in partly milled grains and seeds; $\mathrm{RS}_{2}$ is starch in the form of resistant granules and can be found in raw potato and green bananas; $\mathrm{RS}_{3}$ is retrograded starch that is formed after cooking or baking and is found 
in cooked and cooled potatoes, bread, and cornflakes (167).

The relationship between RS and immune modulation is not documented elaborately in literature. However, some reports have shown the effects of dietary RS in rat models for colitis. $\mathrm{RS}_{3}$ alleviated symptoms in DSS-induced colitis, based mainly on macroscopic and histological parameters, whereas eeFOS did not show significant effects in the same experiment. As both agents stimulated SCFA and butyrate production, it is unclear what caused the different results. Differences in the localization of SCFA production might have played a role: RS induced more butyrate in the cecum and eeFOS induced more butyrate in the distal colon (168). In the same experimental model, it was shown that butyrate uptake by colonic cells was decreased in animals suffering from colitis. RS feeding stimulated butyrate production and at a later time-point also increased its uptake in DSS-treated animals (169). Similar results on SCFA uptake were observed in a TNBS-induced colitis model, showing reduced uptake after colitis uptake without RS supplementation, whereas RS counteracted this effect in the proximal and distal colon (170).

Overall, RS has been shown to reduce intestinal inflammatory parameters that appear to be mediated via microbiota-dependent mechanisms involving SCFAs. At present, there are no data available on systemic immune-modulating effects.

\subsection{Other Non-digestible Carbohydrates}

\subsubsection{Other Oligosaccharides}

Nigerooligosaccharides (short, enzymatically produced (1,3)-linked oligosaccharides consisting of glucose-derived monomers) have been shown to induce immunemodulating effects in various murine models. Increased proliferative capacity was observed after in vitro incubation of lymphocytes with nigerooligosaccharides, as well as increased L. plantarum induced IL-12 production, indicating direct, microbiotaindependent immune-modulatory properties (171). In vitro incubation of murine hepatic mononuclear cells increased NK activity in several mice strains. Application of $1 \%$ nigerooligosaccharides in the drinking water induced similar NK-stimulating results in vivo and improved survival of mice with systemically inoculated tumor cells (172). One article reports effects of nigerooligosaccharides in healthy human volunteers: mitogen responses were enhanced, whereas activation-induced apoptosis was inhibited in PBMC (173).

Isomaltooligosaccharides (short, enzymatically produced (1,6)-linked oligosaccharides consisting of glucose-derived monomers) have shown to induce immune-modulatory effects in mice at a high dietary supplementation dose $(200 \mathrm{~g} / \mathrm{kg})$ for 4 weeks. The 
concentration of $\operatorname{sIg} \mathrm{A}$ in feces was increased, as well as IFN- $\gamma$ production by intraepithelial lymphocytes and liver mononuclear cells. Higher percentages of NKcells were found in the spleen and the liver, and supplementation protected the mice from irradiation-induced lethality (174).

\subsubsection{Other Polysaccharides}

Arabinogalactan from the larch tree is a large branched polysaccharide with a $(3,6)$ linked galactose backbone. It has a food-grade status and is used as a food additive for emulsification, thickening, and so forth. Arabinogalactan structures are also found in many edible and inedible plants, often as a glycoprotein (175). NK activity of different human leukocyte subsets was increased by in vitro incubation with arabinogalactan. In addition, production of IFN- $\gamma$, IL-1 $\beta$, IL-6, and TNF- $\alpha$ was induced in human PBMC in vitro. Preincubation with blocking antibodies to IFN- $\gamma$ and IL-2 inhibited the arabinogalactan-induced NK-boost, suggesting a role for these cytokines (176).

Partially hydrolyzed guar gum alleviated DSS-induced colitis in mice as shown by clinical symptoms, histological analysis, and the fact that mucosal TNF- $\alpha$ production and mRNA expression were partially inhibited (177). In another study, dietary application of guar gum increased MLN IgA, IgG, and $\operatorname{IgM}$ production in aged Sprague-Dawley rats (178).

\section{Mechanism-related observations}

\subsection{Prebiotic Effects}

\subsubsection{Prebiotic Effects of Oligosaccharides}

As mentioned before, HMOS are regarded as the first prebiotic agent that newborns encounter in life that cause the bifidogenic effect of breast milk $(6,14)$. In vitro studies using human and porcine pancreatic enzymes and brush border membranes support the view that HMOS resist hydrolysis in the upper-gastrointestinal tract. Although a small proportion may be absorbed, the majority is thought to reach the large intestine to be fermented by the microbiota (179). HMOS have been shown to be selectively fermented in vitro, supporting the idea that HMOS enhance bacterial growth selectively in vivo (180).

The effects of fructans on the microbiota are well established and have already been demonstrated in human studies in the 1980s (43). Almost $90 \%$ of dietary fructans reach the ileocecal junction, although fecal recovery of these substances is negligible, 
indicating that fermentation mainly takes place in the cecum and colon (181). The induced changes in microbiota are transient; the effects are reversed within a short period after cessation of intake (182). A dose-response relationship between dietary eeFOS and fecal bifidobacteria was observed in a large study (183). Besides effects on the microbiota that are often measured in feces or gut content, a combination of scFOS/lcFOS was shown to increase the amount of bifidobacteria in the layer of microbiota that is associated to the mucosa, a layer of bacteria that is expected to play an important role in host modulation. To a lesser extent, increases in the numbers of lactobacilli and eubacteria were found in the mucosa-associated microbiota (184).

The effects of fructans on the microbiota have been studied in infants as well. Low-dose scFOS supplementation resulted in a significant increase in the number of fecal bifidobacteria, as well as in the percentage of colonized subjects compared with placebo (185). In a study featuring a crossover design and short-term supplementations, scFOS supplementation was shown to increase the numbers of bifidobacteria, parallel to an increase in other bacteria including clostridia (186). Adding scFOS to an infant formula failed to show a significant effect on the total number of bifidobacteria compared with control formula. However, the proportion of bifidobacteria expressed as the percentage of bacteria that were detected by fluorescent in situ hybridization was increased significantly (187). In young children treated with antibiotics, prebiotic treatment with a combination of scFOS/inulin normalized the number of bifidobacteria and lactobacilli (188).

It was concluded in a recent review that different types of fructans induced similar prebiotic effects in humans (189). However, differences in fermentation of scFOS and lcFOS by different strains of bifidobacteria have been reported: all strains of bifidobacteria fermented scFOS, but only a few fermented lcFOS. The different fermentation modes of scFOS and lcFOS resulted in different SCFA profiles in human fecal cultures, with lcFOS resulting mostly in butyrate and scFOS mostly in acetate and lactate production (190). Prebiotic effects and modulation of SCFA profiles or amounts by fructans have been described in many experimental animal models, including mice, rats, and pigs $(191,192)$.

The prebiotic effects of GOS have not been studied as much as fructans and the results vary among different studies. In healthy volunteers with low initial bifido counts, $\beta$ GOS specifically enhanced bifidobacteria counts in fecal samples (193). $\beta$-GOS disaccharides yielded similar results: increasing fecal numbers of lactobacilli and bifidobacteria and decreasing Bacteroidaceae and Candida spp (194). In a non-placebocontrolled study using $10 \mathrm{~g} / \mathrm{d} \beta$-GOS in 8 healthy volunteers, a significant increase in fecal bifidobacteria, but not enterobacteria, was measured (195). In contrast, 
supplementation of $\beta$-GOS in a high-protein diet in healthy volunteers did not result in enhanced fecal numbers of bifidobacteria. However, $\beta$-GOS was not found in fecal samples, indicating that $\beta$-GOS was fermented in the intestine; the authors suggested that this happens in the upper colon. An unexplained result in this study was a significant increase in the number of bifidobacteria in all groups including the placebo group (196).

In rats associated with human fecal microbiota, $\beta$-GOS increased the amount of bifidobacteria, and lactobacilli, as well as total anaerobic bacteria, whereas the number of enterobacteria was decreased, indicating some extent of specificity (197). In a similar experiment, $5 \%$ and $10 \% \beta$-GOS in the diet resulted in increased cecal SCFA production; acetate and propionate, but not butyrate, were increased significantly (198). In pigs, different effects of dietary $\beta$-GOS supplementation have been observed. Supplementation with $\beta$-GOS or scFOS did not affect the number of bifidobacteria or lactobacilli, but trends in SCFA production and glycolytic activities suggested that saccharolytic activity was stimulated in the porcine colon (191). Another report showed that $\beta$-GOS increased colonic numbers of bifidobacteria and SCFA concentrations, mainly caused by increases in acetic acid and consistent with bifidobacterial fermentation (199).

In the same study, $\beta$-GOS inhibited the attachment of intestinal pathogens in vitro, presumably by acting as adhesion receptor decoys (199). Others showed that $\beta$-GOS, as well as a soy oligosaccharide product containing raffinose and stachyose, increased the amounts of bifidobacteria, lactobacilli, and the production of SCFAs in vitro using pig fecal inocula (200). $\alpha$-GOS have been shown in vitro to have a more efficient bifidogenic and lactogenic effect than scFOS and other commercial prebiotics in canine fecal biota cultures (201).

The prebiotic effect of the combination of scGOS/lcFOS (Immunofortis) was tested in infants in multiple placebo-controlled clinical trials. A bifidogenic effect was consistently shown, as well as softer, slightly more frequent stools, and lower fecal $\mathrm{pH}$ in infants (202-205).

In an additional trial, scGOS/lcFOS-supplemented and control formulas were compared with breast feeding with respect to the numbers of fecal bifidobacterial and bacterial metabolites. In subjects fed with the prebiotic mixture, fecal acetate concentrations were higher, whereas propionate and $\mathrm{pH}$ were lower than in controls. Overall, the proportion of bifidobacteria and the metabolic profile in scGOS/lcFOS-fed subjects were more similar to breast-fed subjects than controls (206). In older, formulafed infants that had not received prebiotic treatment before, solid weaning foods containing scGOS/lcFOS enhanced fecal bifidobacterial proportions compared with a 
maltodextrin-containing control diet after weaning (207).

New prebiotic agents are being actively investigated, including enzymatically produced oligosaccharides (200, 208-213), oligosaccharides from natural sources (214), partially hydrolyzed gums (182), fermentations (215), and arabinooligosaccharides (216). It will be interesting to test whether these novel prebiotic agents have immunemodulatory effects.

In conclusion, prebiotic effects of fructans in humans and in animal models have been consistently shown, including stimulation of bifidobacteria and lactobacilli, as well as modulation of bacterial metabolites. This does not mean that all fructans induce the same results; differences in the profiles of produced SCFAs were reported (190). Applied in infant formula, fructan supplementation appears to stimulate bifidobacteria and possibly other bacteria as well. The 9:1 mixture of scGOS/lcFOS has consistently shown bifidogenic effects in infants in multiple clinical trials, whereas experiments with GOS alone showed inconsistent prebiotic and SCFA-modulating effects. Overall, the effects on the intestinal microbiota and their metabolites appear to be different for the various kinds of non-digestible oligosaccharides.

In most studies dealing with immune-modulatory effects of NDCs, effects on the microbiota were not analyzed. Given that modulatory effects on the microbiota have been described in separate experiments, as shown in this section, it is possible that these effects have been causative in the immune-modulating effects that were described in section 2. Issues regarding interaction of microbiota or their metabolites with the host immune system are described in more detail in section 3.2.

\subsubsection{Prebiotic Effects of Polysaccharides}

Immune-modulating effects of $\beta$-glucans are mostly attributed to direct, receptormediated effects on the immune system, probably due to the fact that multiple $\beta$-glucan receptors have been described. However, barley-derived $\beta$-glucan showed clear prebiotic properties in rats, as it increased the proportion of lactobacilli (217). In addition, other authors have described that $\beta$-glucans reach the cecum and colon where they are rapidly fermented (218-220).

Dietary pectin has been shown to modulate the intestinal microbiota. As recently as the 1980s, it was shown that total aerobic and anaerobic bacteria increased in the large intestine after pectin supplementation $(221,222)$. SCFA levels in the cecum of pectin-fed rats were increased, although in this study no effects were observed on cecal flora (223). Pectic oligosaccharides showed prebiotic properties in human in vitro fecal fermentations, showing increases in bifidobacteria as well as Eubacteria. Butyrate production was stimulated as well (224). In rat experiments as well as in in vitro 
fermentation experiments with human fecal flora, it was shown that the degree of esterification influenced SCFA production and speed of fermentation; low esterification resulted in faster fermentation and higher SCFA production $(218,225)$.

Resistant starch is known to modify SCFA production, whereas varying effects on the composition of the microbiota have been reported. Potato RS highly increased butyrate production over a longer period of time, suggesting a slow adaptation process to a diet high in indigestible fibers (226). RS 3 from bananas was shown to be fermented by human fecal flora in vitro resulting in high production of butyrate (227). RS supplementation increased SCFA production in rats, in addition to increased fecal and cecal counts of several bacterial groups including bifidobacteria and lactobacilli. This effect was dependent on the type of RS that was applied (228). Similar effects, showing specific microbiota effects of different RS-containing diets, were observed by other investigators(229) (225), who found that butyrate production in rats was only efficiently stimulated by well-fermentable, but not poorly fermentable, $\mathrm{RS}_{3}$. In rats associated with human flora from donors from the United Kingdom or Italy, dietary RS enhanced butyrate production and increased cecal bifidobacteria, lactobacilli, and enterobacteria (230). Overall, RS have been shown to modulate SCFA production and to have prebiotic effects, depending on the type of RS, and the dietary matrix it is supplied in.

Several other polysaccharides have been shown to affect the microbiota. In humans, larch arabinogalactan was shown to increase fecal lactobacilli regardless of dose, as well as a dose-dependent increase in total anaerobes (231). Guar gum was shown to increase bacterial counts as well and stimulate butyrate production, but not propionate, suggesting fermentative or even prebiotic properties $(232,233)$.

Overall, polysaccharides can clearly modify the microbiota and SCFA production in the large intestine. Although these polysaccharides do not necessarily qualify as prebiotic agents according to the definition of Gibson and Roberfroid (7), it is possible that these microbiota-dependent effects play a role in the immune-modulating effects of orally applied polysaccharides. Given that there are very little data on this subject, more research is needed.

\subsection{Immune-Modulatory Mechanisms Related to Prebiotic Effects}

Many NDCs are fermented in the large intestine, leading to changes in the composition of the microbiota, including stimulation of beneficial bacteria such as bifidobacteria and lactobacilli. These and other groups of bacteria and yeasts have been used as probiotic agents, leading also to changes in the microbiota (234). In addition, NDCs and probiotic agents can both change the production of bacterial metabolites in the intestine. 
Therefore, an indirect mechanism of NDCs or probiotic-induced immune modulation could involve the effects of bacterial metabolites on epithelial and immune cells.

To understand how NDCs potentially modulate the immune system indirectly via "beneficial" bacteria or their metabolites, a condensed review of the relevant literature is included in this section. An overview of the mechanisms leading to microbiotadependent, NDC-induced immune modulation is shown in Figure 7.

\subsubsection{Immune-Modulatory Effects of Probiotic or Commensal Bacteria}

Bacteria obviously have to interact with the immune system in order to modulate it. Janeway proposed in 1989 that pathogens carry pathogen-associated molecular patterns, which are recognized by cells from the innate immune system by means of

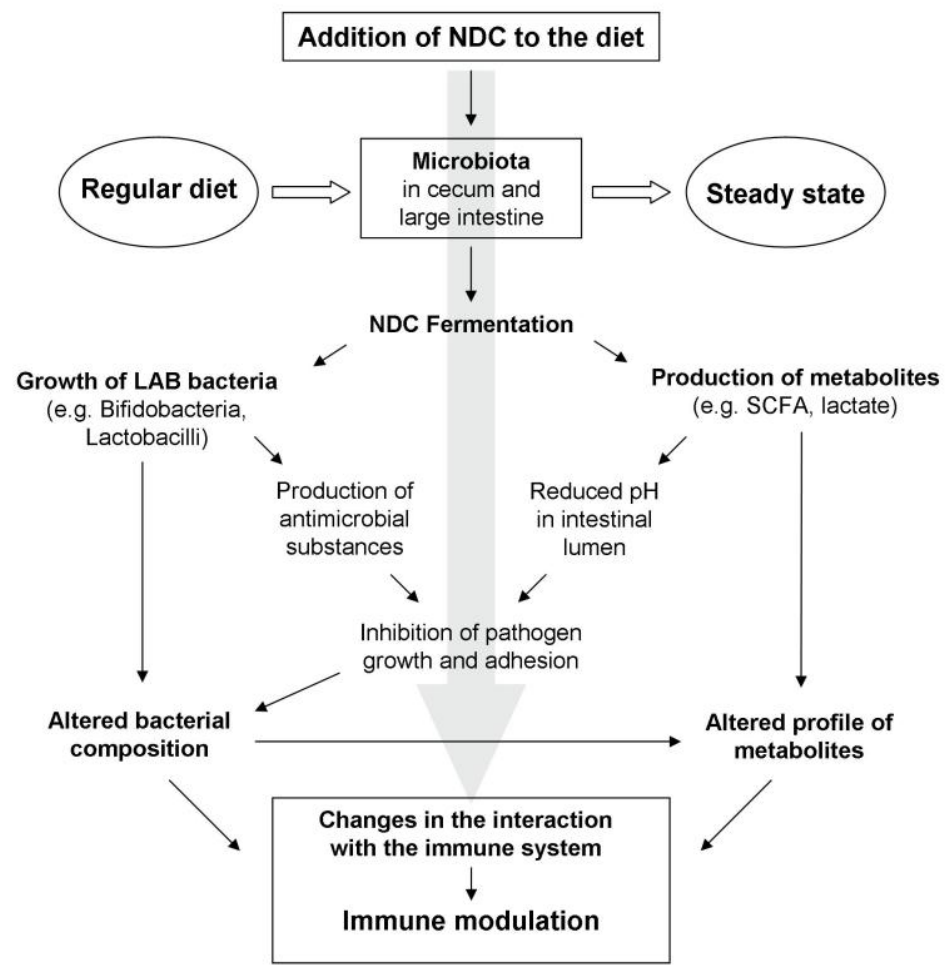

Figure 7. Microbiota-dependent mechanisms of dietary non-digestible carbohydrates (NDC)-induced immune modulation. The horizontal, open arrows represent the situation of a regular diet with a fixed composition, resulting in a steady state regarding substrate fermentation and the composition of the microbiota. The closed, black arrows indicate the effects of the addition of NDCs to the regular diet, leading to immune-modulating effects due to alterations in the bacterial composition (described in section 3.2.1) or to alterations in the profile of bacterial metabolites in the intestine (described in section 3.2.2). 
PRRs (235). TLR and C-type lectins are examples of PRRs that have been identified that are studied widely today. A number of TLRs have been implicated in the interplay between microbiota and the immune system: TLR4 recognizes LPS from Gram-negative bacteria as well as other bacterial and host factors; TLR2 recognizes bacterial substances such as lipoteichoic acid and peptidoglycan from Gram-positive bacteria; and TLR9 recognizes specific bacterial DNA sequences known as CpG-motifs (236). TLRs are expressed by many cells including DCs, monocytes, macrophages, and mucosal epithelial cells (237). Examples of TLR-mediated immune-modulating effects by probiotics have been described. In epithelial cells, probiotic-induced IL-6 production involved TLR2 and TLR4 activation (238). A DNA sequence from a probiotic bacterium was shown to induce systemic immune modulation, skewing the allergic Th2 response more toward a Th1 response. The effect was suggested to be mediated through TLR9 signaling (239). In an elegant study using knockout mice, probiotic DNA was used to ameliorate DSS-induced colitis. The effect was shown to be TLR9-dependent (240).

\subsubsection{In Vitro Effects of Interaction Between Immune Cells and Probiotic Bacteria}

Probiotic bacteria have been shown to modulate the function of immune cells upon direct interaction in vitro. Several studies have shown effects that suggest stimulation of Th1-related responses and/or inhibition of Th2-related responses. Stimulation of IFN- $\gamma$, IL-12, or IL-18 production by probiotics or commensals has been reported in human PBMC and monocytes (241, 242), in human monocyte-derived DCs (243), and in murine macrophages (244). LAB strains, but not E. coli, inhibited Th2-related cytokine production in antigen-specific cultures of human PBMC; the effect was shown to be dependent on the APC in the cultures (245). However, it was also reported that in vivo treatment with probiotics induced the simultaneous stimulation of Th1- and Th2related cytokines ex vivo after sensitization to OVA (246). These data indicate that there are differences between the induction of Th1- and Th2-related cytokines in various cells by probiotics, indicating strain-specific or model-dependent differences in the immunemodulating effect.

Commensal and pathogenic bacteria induced different cytokine signals in cells from mucosal and systemic compartments. Pathogens stimulated Th1-inducing cytokines in both compartments, but commensal bacteria induced Th1-related responses only in systemic immune cells. Commensals stimulated IL-10 production in MLN cells, indicating that commensals induce regulatory signals in the mucosal compartment (247). In other experiments using different Lactobacillus and Bifidobacterium strains, certain strains induced strong IL-10 production in human PBMC (248). Selected probiotic bacteria affected human moDCs to stimulate regulatory $\mathrm{T}$-cell induction, 
stimulating IL-10 production. The IL-10-stimulating effect coincided with DC-SIGN binding of the probiotics, and blocking of DC-SIGN inhibited it. Therefore, DC-SIGN is suggested to play an important role in the induction of regulatory responses, possibly playing an important role in the beneficial effects of (prebiotic-induced) probiotic bacteria in IBD (249). Indeed, protective effects of probiotic lactobacilli were observed in a murine colitis model; however, a non-probiotic lactobacillus strain exacerbated the inflammation, showing strain specificity of the immune-modulating effects of probiotics (250).

\subsubsection{Mechanisms of Interaction In Vivo}

Various mechanisms have been described that can facilitate bacterial modulation of the immune system. Intestinal epithelial cells directly interact with bacteria in the gut and express many PRRs (251). Although these cells are not of immunological origin, they are able to produce inflammatory cytokines and other mediators in response to pathogens $(252,253)$. Several studies have shown that probiotic bacteria do not induce inflammatory responses in intestinal epithelial cells, but are able to inhibit inflammatory responses of these cells to pathogens (254-256). Intestinal epithelial cells may play a role as well, directing the Th1/Th2 balance through production of thymic stromal lymphopoietin. This signaling molecule has been recognized to play a role in the activation of DCs, leading to the attraction of Th2 cells and the polarization of naïve $\mathrm{CD}^{+} \mathrm{T}$ cells into allergic inflammatory cells producing IL-4, $-5,-13$, and TNF- $\alpha$, but not IL-10 (257). Thymic stromal lymphopoietin was shown to be expressed constituently by intestinal epithelial cells, leading to a non-inflammatory state in which mucosal DCs direct $T$ cells toward a non-inflammatory Th2 profile producing IL-4, -5, -13, and IL-10, but not TNF- $\alpha$ (258). In airway epithelial cells, thymic stromal lymphopoietin production was shown to be induced by several bacterial and viral substances (258), suggesting that intestinal expression of the molecule might be a target for modulation by probiotic bacteria.

DCs have been described to penetrate the mucosal barrier to sample bacteria from the lumen. This mechanism was demonstrated in vitro, whereas in vivo experiments yielded consistent results $(259,260)$. In mice, gut sampling of pathogenic and nonpathogenic bacteria by DCs was shown recently to be dependent on the chemokine receptor $\mathrm{CX}_{3} \mathrm{CR} 1$. This gut sampling mechanism was shown to aid in the resistance against invading Salmonella typhimurium, underlining the relevance of the mechanism for immunity in vivo $(261,262)$. In other studies, DCs from PPs and MLNs were shown to have sampled commensal bacteria. Commensal-loaded DCs were shown to be compartmentalized within the mucosal part of the immune system; they were present 
in PPs and MLNs, but were not found in systemic immunological organs like the spleen. This mechanism allows controlled low-level penetration of living commensal bacteria in mucosal immunological tissues and plays a role in protective mucosal IgA responses $(263,264)$. Results found with a zwitterionic polysaccharide from the ubiquitous commensal Bacteroides fragilis may demonstrate how this mechanism can result in profound modulation of systemic immunity. This polysaccharide directed the maturation of the immune system in germ-free mice, restoring systemic $\mathrm{T}$-cell deficiencies and correcting the Th1/Th2 imbalance in these animals. DCs were shown to present the polysaccharide to $\mathrm{T}$ cells in vitro, resulting in signaling, cell activation, and cytokine production. In vivo, the polysaccharide was detectable in MLNs but not in the spleen, resembling the distribution of DCs that have sampled commensal bacteria. It was hypothesized that migrating $\mathrm{T}$ cells relayed the immune-modulating effects from the MLNs to other systemic immunological organs (265).

Microfold (M) cells, overlying PPs in the small intestine, have been known to take up luminal antigens, as well as whole bacteria, in order to present these to the underlying leukocytes. Recently, data were published that suggested that the M-cell bacterial uptake mechanism is mediated by PRRs, including TLR2 and 4. The interplay between PRRs and pathogen-associated molecular patterns is suggested to be decisive for the uptake and subsequent immune response induction, depending on the pathogenicity of the bacterium (266). Villous $M$ cells have been identified that can develop independently of PPs or follicle-associated epithelium. These M cells are also capable of taking up bacteria from the gut, independent of PP tissue (267).

Although there are no specific data about this issue available at present, it can be hypothesized that bacterial uptake mechanisms by DCs and M cells plays a role in systemic and/or mucosal immune modulation by (prebiotic-induced) probiotic bacteria.

\subsubsection{Animal Studies}

Many immune-modulating effects of orally applied probiotics have been reported in animal models, including local as well as systemic effects. Gut-confined protective effects of oral probiotics against enteropathogens have been described for a long time $(268,269)$. In addition, 4 strains of lactobacilli isolated from breast milk were recently shown to have protective properties against oral Salmonella infections (270). However, these protective effects are not necessarily mediated by modulatory effects on the host immune system.

Systemic modulation of immune responses was shown in Listeria monocytogenesinfected rats. DTH responses against the pathogen and resistance to reinfection were enhanced by supplementation with $L$. casei shirota. Proof for the systemic immune- 
modulating effect of the probiotic agent was given by the fact that the stimulation of DTH responses was transferable to untreated mice by adoptive transfer of the splenocytes $(271,272)$.

\subsubsection{Clinical Studies}

There is also evidence that probiotic treatment can modulate immune responses in humans. Beneficial effects of probiotic treatment have been observed on diarrhearelated parameters. Meta-analysis showed that probiotic treatment reduced the incidence of 3-day episodes and the duration in infants and adults (273-277). Mixed results have been obtained in irritable bowel syndrome and IBD patients. Antiinflammatory effects and improved length of remission has been reported; however, the results vary among different disease stages and among studies (278-280).

Probiotic therapy has been tested in a large number of studies for preventing or reducing symptoms of allergy or atopy. Several studies showed improvements of atopic dermatitis disease scores, especially in subjects with high IgE titers (281-285), and partial prevention of atopic disease has been reported as well in a high-risk group of subjects (286). However, this topic is not without controversy and a recent report showed no effects in atopic dermatitis patients (287). In children and young adults suffering from allergic rhinitis, Bacillus clausii was reported to induce a nasal Th $2 \rightarrow$ Th1 cytokine shift and improve other allergy-related parameters in several small clinical trials (288-290). However, probiotics do not always skew the Th1/Th2 balance toward Th1-related responses. A mixture of probiotics was shown to stimulate Th2-related IL-4 production in cow's milk allergic infants ex vivo, whereas L. rhamnosus GG alone stimulated Th1-related IFN- $\gamma$ production (291). These data indicate that different probiotic strains can vary in their immune-modulating effect in humans.

A combination of 2 probiotic strains enhanced the number of cow's milk-specific IgAsecreting cells in formula-fed infants, showing an effect on humoral immunity (292). There are also studies indicating that probiotic treatment may enhance the resistance to infectious disease. A combination of 3 probiotic strains reduced the total symptom score in a common-cold study, as well as duration of episodes and days with fever (293). In healthy volunteers, B. lactis supplementation enhanced phagocytosis of PMN and NK activity, suggesting a stimulation of the nonspecific immune system. Although the study was not placebo-controlled, pretesting with the carrier product did not enhance these parameters (294).

\subsubsection{Immune-Modulatory Effects of Bacterial Metabolites}

NDCs are the predominant substrates for intestinal fermentation, yielding SCFA 
production in non-ruminant species mostly in the cecum and colon. In all species, acetate is the predominant SCFAs found in the large intestine, followed by butyrate and propionate. Much smaller quantities of other metabolites are found as well, including lactate and protein fermentation-derived branched SCFAs such as formate and valerate (295). In humans, the SCFA distribution in the colon was shown to be acetate : butyrate : propionate with a ratio of $60: 19: 21$, respectively (296). Butyrate is an important energy source for enterocytes, whereas the butyrate and propionate that reach portal vein blood are almost completely taken up by the liver. Therefore, butyrate and propionate are suggested to play a significant role in the intestine and liver, but not in the periphery. Hepatic uptake of acetate is around 50\%, allowing significant levels to reach the systemic circulation where it is used as an energy source for host cells and might cause vasodilatory effects $(297,298)$. SCFAs were reported to directly influence gene expression patterns, causing enhanced cell differentiation and reduced proliferation rates in colonocytes, and resulting in suppression or reversal of neoplastic progression. Butyrate was found to be more effective in this respect than propionate or acetate (299).

Of the different SCFAs that are produced by intestinal microbiota, butyrate is most studied and appears to have the most potent modulatory effects on immune cells. Acetate, butyrate, propionate and combinations thereof induced differential effects on mitogen-stimulated rat lymph node cells in vitro. Butyrate was most effective in the inhibition of proliferative responses and stimulation of the regulatory cytokine IL-10, whereas acetate and propionate partially counteracted the effects of butyrate when incubated simultaneously (300). Inhibitory effects on immune parameters have also been shown using human cells in vitro. Butyrate and propionate inhibited proliferation in human PBMC, but it is not clear whether this was caused by inhibition of T cell (301) or APC function (302). In a more recent study, dendritic cell differentiation and maturation were shown to be inhibited by nontoxic levels of butyrate, suggesting immune-suppressive effects of butyrate on APC in a different in vitro test system as well (303).

Inhibitory effects on phagocytosis by PMN and macrophages in vitro have been reported widely. Chemotactic migration to casein, fMLP peptide, and complement component $\mathrm{C} 5 \mathrm{a}$ were found to be inhibited, and PMN phagocytic killing was decreased at concentrations that are readily available in the large intestine (304). Paradoxically, stimulating effects of SCFAs on PMN have been described as well. Propionate and acetate, but not butyrate, induce intracellular calcium influxes in human neutrophils, indicative of activation. Other activating effects, such as a $\mathrm{pH}$ transient and actin alterations, were observed with butyrate as well as the other SCFAs, indicating that 
differential effects occur on different activation parameters $(305,306)$. The literature on these paradoxical effects of SCFAs on PMN is more thoroughly reviewed elsewhere (307). More recently, G-protein-coupled receptors have been described that can be activated by SCFAs and play a role in PMN activation at physiologically relevant SCFA concentrations: G-protein-coupled receptor (GPR)-43, which is expressed in hematopoietic tissues, monocytes, and PMN, and GPR41, which is more broadly expressed in various tissues. These receptors are thought to mediate SCFA-induced effects, whereas high concentrations of SCFAs may induce effects independent of these receptors (308-310).

SCFAs have also been shown to affect immunological properties of epithelial cells. Anti-inflammatory effects were reported in HT-29 and Caco-2 epithelial cell lines, including inhibition of LPS-induced IL-8, monocyte chemoattractant protein-1 production, modulation of TLR4 expression, and altering gene expression by modulation of histone acetylation $(311,312)$. Anti-inflammatory effects of butyrate on epithelial cell lines have been related to the inhibition of NF- $\kappa$ B activity, a mechanism that may play an important role as well in vivo $(313,314)$.

Mucosal epithelial cells produce anti-microbial peptides that are part of the first line of defense against pathogens. Butyrate stimulated anti-microbial cathelicidin expression and stimulated microbicidal activity against S. aureus $(315,316)$. The functional relevance of the effects on anti-microbial peptides is not yet proven in vivo. It was shown that SCFAs increased the PGE1/PGE2 ratio produced by subepithelial myofibroblasts (317). This resulted in an increase in mucus production, which might affect populations of adherent bacteria in the gut. Additionally, modulation of prostaglandin production might affect inflammatory and other immune responses locally.

It is difficult to test SCFAs in vivo because orally applied SCFAs will not reach the physiologically relevant location: the large intestine. Therefore, there are not much data available from animal or clinical studies. Intra-colonic infusions of high concentrations of butyrate and lactate reduced parameters of inflammation in a TNBS-induced colitis model in rats (47). In rats that received various fiber-rich diets, cecal concentrations of SCFAs were shown to be significantly correlated to intraepithelial CD4 ${ }^{+}$and $\mathrm{CD}^{+} \mathrm{T}$ cells, and CD161 ${ }^{+} \mathrm{NK}$ cells; the effects differed among various SCFAs, but acetate and butyrate were both positively correlated to intraepithelial CD8+ T cells (318).

Clinically, butyrate metabolism was shown to be affected in active ulcerative colitis; oxidation of butyrate in colonocytes was reduced (319), whereas administration of butyrate enemas improved the clinical course in ulcerative colitis patients (320-322).

Besides SCFAs, it has been suggested that other metabolites from the microbiota might 
modulate the host immune system as well. Small peptides with immune-modulating capacity have been shown to be produced during anaerobe culture of several commensal bacteria, including lactobacilli. It was described that oral application of these bacterial peptides reversed antibiotic-induced immune suppression and proliferation of lymphatic tissue in mice. Although the peptides were not characterized, and it is not known whether the production of these peptides is affected by fermentation of NDCs, it is possible that they play a role in bacterially-induced immune modulation (323-325).

\subsection{Direct Interactions: Lectins}

Apart from microbiota-dependent mechanisms, NDCs may modulate the immune system through direct interactions with receptors or other molecules that relay immunological messages.

Lectins are carbohydrate-binding proteins (excluding enzymes and immunoglobulins) with a wide variety of functions, including PRR activity. Ten families of animal lectins are distinguished on the basis of structure homology, including calcium-dependent Ctype lectins, S-type lectins (galectins), P-type lectins, and others, featuring a huge variety in sugar-binding activities in total. The affinity for individual saccharide ligands is usually low, but higher affinity binding is observed with multiple binding sitecontaining ligands, a phenomenon called the glycoside cluster effect $(326,327)$. Galectins are $\beta$-galactoside or $\mathrm{N}$-acetyllactosamine binding proteins that are mostly non-membrane bound; therefore, they cannot be classified as PRRs, but they do appear to modulate immune function $(328,329)$. As was described above, it has been suggested in the literature that pectin-derivates can modulate galectin-3 function, resulting in anti-tumor effects in vivo (156-158).

It is interesting to note that polysaccharide binding and activation of TLR4, a non-lectin receptor, are reported as well $(330,331)$.

\subsubsection{Brief Overview of Potential Interactions Between Lectins and Non-digestible Carbohydrates}

Although glycobiology is a highly active research discipline nowadays, there are large gaps in the knowledge about the functions of the wide variety of lectins and their respective sugar specificities. A full review of this large and rapid-growing research area is not included here, but it should be noted that the various types of lectins known today are potential targets for NDC-induced immune modulation. Such modulation could be induced by dietary carbohydrates that bind lectins in a way that involves 
receptor activation. However, if carbohydrates bind lectins without activating them, immune modulation could also occur by effectively blocking the lectin from its normal ligand. If such mechanisms are indeed relevant in vivo, dietary carbohydrates must be able to interact with lectins. Several cell types and mechanisms that could potentially play a role are described briefly.

DCs play a central role in immunity and tolerance. Together with other APC they express high levels of C-type lectins, which are differentially expressed in different various subsets (332). C-type lectins on DCs participate in cell-cell adhesion, pattern recognition, and antigen uptake for MHC-presentation (333-335). Because DCs are known to sample gut contents (259-262), NDC-DC interactions may occur in the gut.

Epithelial cells also express a wide variety of PRRs, including lectins (251, 336). Therefore, they might also play a role in NDC-induced immune modulation.

$\mathrm{M}$ cells take up soluble antigens as well as (micro) particles from the gut lumen. NDCs could be taken up by $\mathrm{M}$ cells and subsequently come into contact with DCs, T, or B cells in PPs, or homologue tissues. Alternatively, luminal carbohydrates could modulate binding and/or uptake of other molecules or particles by M cells (337).

There are indications in literature that small fractions of ingested oligosaccharides enter the circulation. In eeFOS-supplemented healthy volunteers, approximately $0.1 \%$ of the ingested oligosaccharides were recovered in urine. The distribution of fructan chainlengths in urine closely resembled the distribution of chain-lengths in the ingested material, which suggests that the systemic availability of small oligosaccharides (range of $\mathrm{dp} 2-4$ ) does not depend on the size of the molecule (338). In addition, HMOS have been found in the urine of breast-fed infants (23). These data indicate that the gut barrier is not absolute for all NDCs; minute fractions of ingested carbohydrates may enter the systemic circulation and potentially modulate immune cells that express lectins. As mentioned before, HMOS have been described to bind or affect expression of selectins $(25,26)$, DC-SIGN (27), integrins (28, 29), and galectins (30). These molecules are also potential targets for binding to other NDCs in the intestine or systemically. In addition, cytokine functions might be modulated by NDCs, as many cytokines possess carbohydrate-binding domains that are suggested to be relevant for their immunological functions $(327,339)$.

\subsubsection{Lectins: Summarizing Remarks}

In conclusion, carbohydrates could exert immune-modulatory properties locally in the gut through interaction with PRRs on many cell types that can directly come into contact with gut content. In addition, small fractions of multimeric sugar molecules might pass the gut barrier and exert immune-modulatory effects systemically. 
Although these mechanisms are highly speculative, there is some evidence for systemic immune-modulating effects of orally applied NDCs that are microbiota-independent $(89,91)$.

\section{Conclusions}

A wide variety of dietary NDCs have been reported to modulate immune functions in experimental animals and man. These NDCs range from small, enzymatically produced oligosaccharides to large polysaccharides that occur naturally in the diet. Local effects of NDC supplementation have been observed in the intestine in many different models, including effects on intestinal inflammation, tumor formation, mucosal immune responses, and mucosal cell populations. Systemic modulation of immune responses has been reported as well after dietary administration of NDCs, including enhanced resistance to systemic infections, enhanced responses to systemic vaccination, and a reduced incidence of atopy. NDCs have been studied mostly in animals or in vitro. Although beneficial immune-modulating effects have been demonstrated in a limited number of clinical studies, more trials are needed to confirm the preclinical results in humans.

The reported effects on the immune system include modulation of specific and nonspecific responses, cellular and humoral responses, and mucosal and systemic responses. Some NDCs induce immune-modulating activities that might appear contradictory at first glance; for example, fructans were described to induce antiinflammatory effects in colitis models (45-48), whereas immune-stimulating effects were shown in enteric or systemic infection models (52). However, these data are not in direct conflict because the immunological mechanisms that play a role in the different models are different. In contrast, conflicting evidence is present in a few other cases. For example, contradictory effects of $\beta$-glucans were reported on inflammatory responses and TNF- $\alpha$ production in macrophages $(121,123-126)$. Because many NDCs contain a mixture of different carbohydrate molecules that may vary among different preparations from the same source, it is unclear whether these discrepancies are due to variation between experiments and experimenters or to variation in NDC preparations. Given that orally applied NDCs co-locate in the gastrointestinal tract with the rest of the diet, potential interactions between NDCs and the nutritional matrix need to be considered as well.

Many potential mechanisms of NDC-induced immune modulation have been described in this review, but in most cases the available data are not conclusive about 
the relevance of these mechanisms in vivo. Results from animal experiments with orally applied $\beta$-GOS and raffinose strongly suggested that these systemic immunemodulation effects were induced in a microbiota-independent way $(89,91)$. In experimentally induced colitis, the results indicated that the amelioration of disease symptoms by fructans was caused by a microbiota-dependent mechanism $(47,48)$. The underlying mechanisms were not clarified in other in vivo studies; in most cases, no attempt was made to do so. Various authors have shown immune-modulatory effects simultaneously to effects on the microbiota; however, this does not provide conclusive proof of a causative relation between both effects. To gain more insight into the mechanisms of NDC-induced immune modulation, it is important that the effects of NDCs are investigated in vitro, in vivo, and in clinical studies, an approach that is advocated by the International Life Sciences Institute (ILSI) expert-group as well (340). Nonetheless, interesting parallels have been observed between immune-modulatory effects of different NDCs, probiotics, and SCFAs. Breast feeding has been reported to lower the incidence of atopy-related disorders (35-37), an effect that was also shown for the scGOS/lcFOS mixture of oligosaccharides (Immunofortis) designed to mimic the function of HMOS in infant formula (97). In addition, similar results were reported in several studies with probiotics on atopy- and allergy-related parameters (281-286). These data might suggest that the anti-atopic effects were caused by microbiotadependent mechanisms, as the growth and/or colonization of beneficial bacteria was stimulated by breast feeding, and scGOS/lcFOS, as well as probiotic treatment.

Oral application of several types of NDCs, including fructans (45-48) and RS (168), as well as several probiotics $(239,240)$, and local application of SCFAs (320-322), have been shown to reduce inflammation or clinical symptoms in IBD-related studies. Seeing that the effects appeared to be mediated by the presence of specific intestinal bacteria as well as their fermentation metabolites, a synbiotic approach combining specific strains of beneficial bacteria and their substrate may be promising in this line of research.

Fructans are very different from $\beta$-glucans with respect to their carbohydrate backbones and chain lengths. However, both groups of NDCs have shown similar effects in vitro on IFN- $\gamma$ - primed macrophages $(44,127)$ and on activation of the alternative complement pathway, which is only induced by insoluble forms of these NDCs (79-82). More research is needed to determine whether both types of glucans share a common microbiota-independent pathway of immune modulation that explains these results. The role of microbiota-dependent mechanisms in $\beta$-glucaninduced immune modulation has not been investigated, but there are indications that $\beta$-glucans have prebiotic properties (217-220). Moreover, an orally applied $\beta$-glucan induced a similar shift toward Th1-related cytokine production in nasal lavage fluid of 
allergic individuals (120), as was reported for treatment with a probiotic bacterium (288-290). These data might suggest a role for a microbiota-dependent mechanism of immune modulation by $\beta$-glucans, but it remains to be determined whether such a mechanism is functionally relevant.

Protective effects of various NDCs have been observed in intestinal tumor models, including the effects of fructans $(52,53,56,57)$ and $\beta$-glucans $(141)$. These effects may be caused by NDC-induced SCFA production, as SCFAs have been shown to inhibit proliferation and stimulate differentiation of colonic epithelial cells (299). However, anti-carcinogenic effects of fructans $(58,59,84)$, $\beta$-glucans $(142,143)$, pectins $(157,158)$, and nigerooligosaccharides (172) have been reported in systemic tumor models as well. It is unknown what mechanisms cause these effects, but it is tempting to speculate that the immune system is involved.

The previous general observations offer some insight into similarities and possible mechanisms regarding effects of various NDCs and probiotics on the immune system; however, caution should be taken in making general statements. Various structurally related NDCs, such as different fructans, were shown to induce differential effects on functional immune parameters in vivo and on microbiota-related parameters $(52,190$, chapter 3). For probiotic bacteria, strain-specific effects have been reported in many studies, including effects on functional immune parameters in vitro and in vivo (248, 250). This indicates that there is a specificity in the effects of particular agents, which is observed both in the field of NDCs and prebiotics as in the fields of probiotics and synbiotics.

In conclusion, many reports have demonstrated mucosal and systemic immunemodulatory effects induced by orally applied NDCs, but more research is needed to identify and understand the underlying working mechanisms and to confirm the results in clinical studies. It is important that experiments with NDCs, whether these are regarded to be prebiotic or not, are performed with an open mind for potential microbiota-dependent and -independent effects on the immune system.

\section{References}

1. Tungland, B.C. and D. Meyer, Nondigestible oligo- and polysaccharides (dietary fiber): their physiology and role in human health and food. Comp Rev Food Sci Food Safety, 2002. 1(3): p. 73-92.

2. Green, C.J., Fiber in enteral nutrition. S A J Clin Nutr, 2000. 13(4): p. 150-160.

3. Boehm, G. and B. Stahl, Oligosaccharides, in Functional dairy products, T. Mattila-Sandholm, Editor. 2003, Woodhead: Cambridge. p. 203-43. 
4. Tissier, H., Repartition des microbes dans l'intestin du nourisson. Ann de l'Institut de Pasteur, 1905. 19: p. 109.

5. Gyorgy, P., R.W. Jeanloz, H. von Nicolai, and F. Zilliken, Undialyzable growth factors for Lactobacillus bifidus var. pennsylvanicus. Protective effect of sialic acid bound to glycoproteins and oligosaccharides against bacterial degradation. Eur J Biochem, 1974. 43(1): p. 29-33.

6. Coppa, G.V., S. Bruni, L. Morelli, S. Soldi, and O. Gabrielli, The first prebiotics in humans: human milk oligosaccharides. J Clin Gastroenterol, 2004. 38(6 Suppl): p. S80-3.

7. Gibson, G.R. and M.B. Roberfroid, Dietary modulation of the human colonic microbiota: introducing the concept of prebiotics. J Nutr, 1995. 125(6): p. 1401-12.

8. $\quad \mathrm{FAO} / \mathrm{WHO}$, Evaluation of health and nutritional properties of probiotics in food including powder milk with live lactic acid bacteria., in Report of a Joint FAO/WHO Expert Consultation. 2001: Available at http://www.who.int/foodsafety/publications/fs_management/en/probiotics.pdf.

9. Lull, C., H.J. Wichers, and H.F. Savelkoul, Antiinflammatory and immunomodulating properties of fungal metabolites. Mediators Inflamm, 2005. 2005(2): p. 63-80.

10. Bohn, J.A. and J.N. BeMiller, (1-->3)-beta-D-Glucans as biological response modifiers: a review of structure-functional activity relationships. Carbohydr Polym, 1995. 28: p. 3-14.

11.

Stahl, B., S. Thurl, J. Zeng, M. Karas, F. Hillenkamp, M. Steup, and G. Sawatzki, Oligosaccharides from human milk as revealed by matrix-assisted laser desorption/ionization mass spectrometry. Anal Biochem, 1994. 223(2): p. 218-26.

12.

Finke, B., B. Stahl, A. Pfenninger, M. Karas, H. Daniel, and G. Sawatzki, Analysis of high-molecularweight oligosaccharides from human milk by liquid chromatography and MALDI-MS. Anal Chem, 1999. 71(17): p. 3755-62.

13. Pfenninger, A., M. Karas, B. Finke, B. Stahl, and G. Sawatzki, Mass spectrometric investigations of human milk oligosaccharides. Adv Exp Med Biol, 2001. 501: p. 279-84.

14. Newburg, D.S., G.M. Ruiz-Palacios, and A.L. Morrow, Human milk glycans protect infants against enteric pathogens. Annu Rev Nutr, 2005. 25: p. 37-58.

15. Bode, L., Recent advances on structure, metabolism, and function of human milk oligosaccharides. J Nutr, 2006. 136(8): p. 2127-30.

16. Coppa, G.V., L. Zampini, T. Galeazzi, B. Facinelli, L. Ferrante, R. Capretti, and G. Orazio, Human milk oligosaccharides inhibit the adhesion to Caco-2 cells of diarrheal pathogens: Escherichia coli, Vibrio cholerae, and Salmonella fyris. Pediatr Res, 2006. 59(3): p. 377-82.

17. Angeloni, S., J.L. Ridet, N. Kusy, H. Gao, F. Crevoisier, S. Guinchard, S. Kochhar, H. Sigrist, and N. Sprenger, Glycoprofiling with micro-arrays of glycoconjugates and lectins. Glycobiology, 2005. 15(1): p. 31-41.

18. Chaturvedi, P., C.D. Warren, M. Altaye, A.L. Morrow, G. Ruiz-Palacios, L.K. Pickering, and D.S. Newburg, Fucosylated human milk oligosaccharides vary between individuals and over the course of lactation. Glycobiology, 2001. 11(5): p. 365-72.

19. Thurl, S., J. Henker, M. Siegel, K. Tovar, and G. Sawatzki, Detection of four human milk groups with respect to Lewis blood group dependent oligosaccharides. Glycoconj J, 1997. 14(7): p. 795-9.

20. Newburg, D.S., G.M. Ruiz-Palacios, M. Altaye, P. Chaturvedi, J. Meinzen-Derr, L. Guerrero Mde, and A.L. Morrow, Innate protection conferred by fucosylated oligosaccharides of human milk against diarrhea in breastfed infants. Glycobiology, 2004. 14(3): p. 253-63.

21. Rothenbacher, D., M. Weyermann, G. Bode, M. Kulaksiz, B. Stahl, and H. Brenner, Role of Lewis A and Lewis B blood group antigens in Helicobacter pylori infection. Helicobacter, 2004. 9(4): p. 324-9.

22. Chaturvedi, P., C.D. Warren, C.R. Buescher, L.K. Pickering, and D.S. Newburg, Survival of human milk oligosaccharides in the intestine of infants. Adv Exp Med Biol, 2001. 501: p. 315-23. 
23. Obermeier, S., S. Rudloff, G. Pohlentz, M.J. Lentze, and C. Kunz, Secretion of 13 C-labelled oligosaccharides into human milk and infant's urine after an oral [13C]galactose load. Isotopes Environ Health Stud, 1999. 35(1-2): p. 119-25.

24. Eiwegger, T., B. Stahl, J. Schmitt, G. Boehm, M. Gerstmayr, J. Pichler, E. Dehlink, C. Loibichler, R. Urbanek, and Z. Szepfalusi, Human milk--derived oligosaccharides and plant-derived oligosaccharides stimulate cytokine production of cord blood T-cells in vitro. Pediatr Res, 2004.56(4): p. 536-40.

25. Schumacher, G., G. Bendas, B. Stahl, and C. Beermann, Human milk oligosaccharides affect Pselectin binding capacities: in vitro investigation. Nutrition, 2006. 22(6): p. 620-7.

26. Rudloff, S., C. Stefan, G. Pohlentz, and C. Kunz, Detection of ligands for selectins in the oligosaccharide fraction of human milk. Eur J Nutr, 2002. 41(2): p. 85-92.

27. Naarding, M.A., I.S. Ludwig, F. Groot, B. Berkhout, T.B. Geijtenbeek, G. Pollakis, and W.A. Paxton, Lewis X component in human milk binds DC-SIGN and inhibits HIV-1 transfer to CD4+ T lymphocytes. J Clin Invest, 2005. 115(11): p. 3256-64.

28. Pabst, H.F., D.W. Spady, L.M. Pilarski, M.M. Carson, J.A. Beeler, and M.P. Krezolek, Differential modulation of the immune response by breast- or formula-feeding of infants. Acta Paediatr, 1997. 86(12): p. 1291-7.

29. Bode, L., S. Rudloff, C. Kunz, S. Strobel, and N. Klein, Human milk oligosaccharides reduce plateletneutrophil complex formation leading to a decrease in neutrophil beta 2 integrin expression. J Leukoc Biol, 2004. 76(4): p. 820-6.

30. Di Virgilio, S., J. Glushka, K. Moremen, and M. Pierce, Enzymatic synthesis of natural and $13 \mathrm{C}$ enriched linear poly- $\mathrm{N}$-acetyllactosamines as ligands for galectin-1. Glycobiology, 1999. 9(4): p. 35364 .

31. Pickering, L.K., D.M. Granoff, J.R. Erickson, M.L. Masor, C.T. Cordle, J.P. Schaller, T.R. Winship, C.L. Paule, and M.D. Hilty, Modulation of the immune system by human milk and infant formula containing nucleotides. Pediatrics, 1998. 101(2): p. 242-9.

32. Blaymore Bier, J.A., T. Oliver, A. Ferguson, and B.R. Vohr, Human milk reduces outpatient upper respiratory symptoms in premature infants during their first year of life. J Perinatol, 2002. 22(5): $p$. 354-9.

33. Cushing, A.H., J.M. Samet, W.E. Lambert, B.J. Skipper, W.C. Hunt, S.A. Young, and L.C. McLaren, Breastfeeding reduces risk of respiratory illness in infants. Am J Epidemiol, 1998. 147(9): p. 863-70.

34. Sinha, A., J. Madden, D. Ross-Degnan, S. Soumerai, and R. Platt, Reduced risk of neonatal respiratory infections among breastfed girls but not boys. Pediatrics, 2003. 112(4): p. e303.

35. Gdalevich, M., D. Mimouni, M. David, and M. Mimouni, Breast-feeding and the onset of atopic dermatitis in childhood: a systematic review and meta-analysis of prospective studies. J Am Acad Dermatol, 2001. 45(4): p. 520-7.

36. Gdalevich, M., D. Mimouni, and M. Mimouni, Breast-feeding and the risk of bronchial asthma in childhood: a systematic review with meta-analysis of prospective studies. J Pediatr, 2001. 139(2): p. 261-6.

37. van Odijk, J., I. Kull, M.P. Borres, P. Brandtzaeg, U. Edberg, L.A. Hanson, A. Host, M. Kuitunen, S.F. Olsen, S. Skerfving, J. Sundell, and S. Wille, Breastfeeding and allergic disease: a multidisciplinary review of the literature (1966-2001) on the mode of early feeding in infancy and its impact on later atopic manifestations. Allergy, 2003. 58(9): p. 833-43.

38. Cani, P.D., C. Knauf, M.A. Iglesias, D.J. Drucker, N.M. Delzenne, and R. Burcelin, Improvement of glucose tolerance and hepatic insulin sensitivity by oligofructose requires a functional glucagon-like peptide 1 receptor. Diabetes, 2006. 55(5): p. 1484-90.

39. Beylot, M., Effects of inulin-type fructans on lipid metabolism in man and in animal models. Br J Nutr, 2005. 93 Suppl 1: p. S163-8. 
40. Weaver, C.M., Inulin, oligofructose and bone health: experimental approaches and mechanisms. Br J Nutr, 2005. 93 Suppl 1: p. S99-103.

41. Coussement, P.A., Inulin and oligofructose: safe intakes and legal status. J Nutr, 1999. 129(7 Suppl): p. $1412 \mathrm{~S}-7 \mathrm{~S}$.

42. van Loo, J., P. Coussement, L. de Leenheer, H. Hoebregs, and G. Smits, On the presence of inulin and oligofructose as natural ingredients in the western diet. Crit Rev Food Sci Nutr, 1995. 35(6): p. 525-52.

43. Mitsuoka, T., H. Hidaka, and T. Eida, Effect of fructo-oligosaccharides on intestinal microflora. Nahrung, 1987. 31(5-6): p. 427-36.

44. Koo, H.N., S.H. Hong, H.G. Seo, T.S. Yoo, K.N. Lee, N.S. Kim, C.H. Kim, and H.M. Kim, Inulin stimulates NO synthesis via activation of PKC-alpha and protein tyrosine kinase, resulting in the activation of NF-kappaB by IFN-gamma-primed RAW 264.7 cells. J Nutr Biochem, 2003. 14(10): p. 598-605.

45. Lara-Villoslada, F., O. de Haro, D. Camuesco, M. Comalada, J. Velasco, A. Zarzuelo, J. Xaus, and J. Galvez, Short-chain fructooligosaccharides, in spite of being fermented in the upper part of the large intestine, have anti-inflammatory activity in the TNBS model of colitis. Eur J Nutr, 2006.

46. Hoentjen, F., G.W. Welling, H.J. Harmsen, X. Zhang, J. Snart, G.W. Tannock, K. Lien, T.A. Churchill, M. Lupicki, and L.A. Dieleman, Reduction of colitis by prebiotics in HLA-B27 transgenic rats is associated with microflora changes and immunomodulation. Inflamm Bowel Dis, 2005. 11(11): p. 977 85 .

47. Cherbut, C., C. Michel, and G. Lecannu, The prebiotic characteristics of fructooligosaccharides are necessary for reduction of TNBS-induced colitis in rats. J Nutr, 2003. 133(1): p. 21-7.

48. Videla, S., J. Vilaseca, M. Antolin, A. Garcia-Lafuente, F. Guarner, E. Crespo, J. Casalots, A. Salas, and J.R. Malagelada, Dietary inulin improves distal colitis induced by dextran sodium sulfate in the rat. Am J Gastroenterol, 2001. 96(5): p. 1486-93.

49. Seksik, P., H. Sokol, P. Lepage, N. Vasquez, C. Manichanh, I. Mangin, P. Pochart, J. Dore, and P. Marteau, Review article: the role of bacteria in onset and perpetuation of inflammatory bowel disease. Aliment Pharmacol Ther, 2006. 24 Suppl 3: p. 11-8.

50. Neyrinck, A.M., H. Alexiou, and N.M. Delzenne, Kupffer cell activity is involved in the hepatoprotective effect of dietary oligofructose in rats with endotoxic shock. J Nutr, 2004. 134(5): p. 1124-9.

51. Milo, L.A., N.J. Correa-Matos, S.M. Donovan, and K.A. Tappenden, Neutrophil and small intestinal lymphocyte migration after Salmonella typhimurium infection: impact of fermentable fiber. J Pediatr Gastroenterol Nutr, 2004.39(1): p. 73-9.

52. Buddington, K.K., J.B. Donahoo, and R.K. Buddington, Dietary oligofructose and inulin protect mice from enteric and systemic pathogens and tumor inducers. J Nutr, 2002. 132(3): p. 472-7.

53. Pierre, F., P. Perrin, M. Champ, F. Bornet, K. Meflah, and J. Menanteau, Short-chain fructooligosaccharides reduce the occurrence of colon tumors and develop gut-associated lymphoid tissue in Min mice. Cancer Res, 1997. 57(2): p. 225-8.

54. Pierre, F., P. Perrin, E. Bassonga, F. Bornet, K. Meflah, and J. Menanteau, T cell status influences colon tumor occurrence in min mice fed short chain fructo-oligosaccharides as a diet supplement. Carcinogenesis, 1999. 20(10): p. 1953-6.

55. Mutanen, M., A.M. Pajari, and S.I. Oikarinen, Beef induces and rye bran prevents the formation of intestinal polyps in Apc(Min) mice: relation to beta-catenin and PKC isozymes. Carcinogenesis, 2000. 21(6): p. 1167-73.

56. Femia, A.P., C. Luceri, P. Dolara, A. Giannini, A. Biggeri, M. Salvadori, Y. Clune, K.J. Collins, M. Paglierani, and G. Caderni, Antitumorigenic activity of the prebiotic inulin enriched with oligofructose in combination with the probiotics Lactobacillus rhamnosus and Bifidobacterium lactis on azoxymethane-induced colon carcinogenesis in rats. Carcinogenesis, 2002. 23(11): p. 1953-60. 
57. Roller, M., A. Pietro Femia, G. Caderni, G. Rechkemmer, and B. Watzl, Intestinal immunity of rats with colon cancer is modulated by oligofructose-enriched inulin combined with Lactobacillus rhamnosus and Bifidobacterium lactis. Br J Nutr, 2004. 92(6): p. 931-8.

58. Taper, H.S. and M.B. Roberfroid, Inulin/oligofructose and anticancer therapy. Br J Nutr, 2002. 87 Suppl 2: p. S283-6.

59. Taper, H.S. and M.B. Roberfroid, Possible adjuvant cancer therapy by two prebiotics--inulin or oligofructose. In Vivo, 2005. 19(1): p. 201-4.

6o. Roller, M., G. Rechkemmer, and B. Watzl, Prebiotic inulin enriched with oligofructose in combination with the probiotics Lactobacillus rhamnosus and Bifidobacterium lactis modulates intestinal immune functions in rats. J Nutr, 2004. 134(1): p. 153-6.

61. Hosono, A., A. Ozawa, R. Kato, Y. Ohnishi, Y. Nakanishi, T. Kimura, and R. Nakamura, Dietary fructooligosaccharides induce immunoregulation of intestinal IgA secretion by murine Peyer's patch cells. Biosci Biotechnol Biochem, 2003. 67(4): p. 758-64.

62. Manhart, N., A. Spittler, H. Bergmeister, M. Mittlbock, and E. Roth, Influence of fructooligosaccharides on Peyer's patch lymphocyte numbers in healthy and endotoxemic mice. Nutrition, 2003. 19(7-8): p. 657-6o.

63. Nakamura, Y., S. Nosaka, M. Suzuki, S. Nagafuchi, T. Takahashi, T. Yajima, N. Takenouchi-Ohkubo, T. Iwase, and I. Moro, Dietary fructooligosaccharides up-regulate immunoglobulin A response and polymeric immunoglobulin receptor expression in intestines of infant mice. Clin Exp Immunol, 2004. 137(1): p. 52-8.

64. Lindsay, J.O., K. Whelan, A.J. Stagg, P. Gobin, H.O. Al-Hassi, N. Rayment, M.A. Kamm, S.C. Knight, and A. Forbes, Clinical, microbiological, and immunological effects of fructo-oligosaccharide in patients with Crohn's disease. Gut, 2006. 55(3): p. 348-55.

65. Welters, C.F., E. Heineman, F.B. Thunnissen, A.E. van den Bogaard, P.B. Soeters, and C.G. Baeten, Effect of dietary inulin supplementation on inflammation of pouch mucosa in patients with an ileal pouch-anal anastomosis. Dis Colon Rectum, 2002. 45(5): p. 621-7.

66. Furrie, E., S. Macfarlane, A. Kennedy, J.H. Cummings, S.V. Walsh, A. O'Neil D, and G.T. Macfarlane, Synbiotic therapy (Bifidobacterium longum/Synergy 1) initiates resolution of inflammation in patients with active ulcerative colitis: a randomised controlled pilot trial. Gut, 2005. 54(2): p. 242-9.

67. Bunout, D., S. Hirsch, M. Pia de la Maza, C. Munoz, F. Haschke, P. Steenhout, P. Klassen, G. Barrera, V. Gattas, and M. Petermann, Effects of prebiotics on the immune response to vaccination in the elderly. JPEN J Parenter Enteral Nutr, 2002. 26(6): p. 372-6.

68. Cummings, J.H., S. Christie, and T.J. Cole, A study of fructo oligosaccharides in the prevention of travellers' diarrhoea. Aliment Pharmacol Ther, 2001. 15(8): p. 1139-45.

69. Lewis, S., S. Burmeister, and J. Brazier, Effect of the prebiotic oligofructose on relapse of Clostridium difficile-associated diarrhea: a randomized, controlled study. Clin Gastroenterol Hepatol, 2005. 3(5): p. 442-8.

70. Ten Bruggencate, S.J., I.M. Bovee-Oudenhoven, M.L. Lettink-Wissink, and R. Van der Meer, Dietary fructo-oligosaccharides dose-dependently increase translocation of salmonella in rats. J Nutr, 2003. 133(7): p. 2313-8.

71. Ten Bruggencate, S.J., I.M. Bovee-Oudenhoven, M.L. Lettink-Wissink, M.B. Katan, and R. Van Der Meer, Dietary fructo-oligosaccharides and inulin decrease resistance of rats to salmonella: protective role of calcium. Gut, 2004. 53(4): p. 530-5.

72.

Ten Bruggencate, S.J., I.M. Bovee-Oudenhoven, M.L. Lettink-Wissink, and R. Van der Meer, Dietary fructooligosaccharides increase intestinal permeability in rats. J Nutr, 2005. 135(4): p. 837-42.

73. Ten Bruggencate, S.J., I.M. Bovee-Oudenhoven, M.L. Lettink-Wissink, M.B. Katan, and R. van der Meer, Dietary fructooligosaccharides affect intestinal barrier function in healthy men. J Nutr, 2006. 136(1): p. 70-4. 
74. Scholtens, P.A., M.S. Alles, L.E. Willemsen, C. van den Braak, J.G. Bindels, G. Boehm, and M.J. Govers, Dietary fructo-oligosaccharides in healthy adults do not negatively affect faecal cytotoxicity: a randomised, double-blind, placebo-controlled crossover trial. Br J Nutr, 2006. 95(6): p. 1143-9.

75. Duggan, C., M.E. Penny, P. Hibberd, A. Gil, A. Huapaya, A. Cooper, F. Coletta, C. Emenhiser, and R.E. Kleinman, Oligofructose-supplemented infant cereal: 2 randomized, blinded, community-based trials in Peruvian infants. Am J Clin Nutr, 2003. 77(4): p. 937-42.

76. Saavedra, J.M. and A. Tschernia, Human studies with probiotics and prebiotics: clinical implications. Br J Nutr, 2002. 87 Suppl 2: p. S241-6.

77. Waligora-Dupriet, A.J., F. Campeotto, I. Nicolis, A. Bonet, P. Soulaines, C. Dupont, and M.J. Butel, Effect of oligofructose supplementation on gut microflora and well-being in young children attending a day care centre. Int J Food Microbiol, 2006.

78. Firmansyah, A., G. Pramita, A. Carrie Fassler, F. Haschke, and H. Link-Amster, Improved humoral immune response to measles vaccine in infants receiving infant cereal with fructooligosaccharides. J Pediatr Gastroenterol Nutr, 2001. 31: p. A521.

79. Silva, D.G., P.D. Cooper, and N. Petrovsky, Inulin-derived adjuvants efficiently promote both Th1 and Th2 immune responses. Immunol Cell Biol, 2004. 82(6): p. 611-6.

8o. Cooper, P.D., Solid phase activators of the alternative pathway of complement and their use in vivo, in Activators and inhibitors of complement, R.B. Sim, Editor. 1993, Kluwer Academic Publishers: Dordrecht. p. 99-106.

81. Cooper, P.D. and M. Carter, Anti-complementary action of polymorphic "solubility forms" of particulate inulin. Mol Immunol, 1986. 23(8): p. 895-901.

82. Czop, J.K. and K.F. Austen, Properties of glycans that activate the human alternative complement pathway and interact with the human monocyte beta-glucan receptor. J Immunol, 1985. 135(5): p. 3388-93.

83. Kerekes, K., P.D. Cooper, J. Prechl, M. Jozsi, Z. Bajtay, and A. Erdei, Adjuvant effect of gamma-inulin is mediated by $C_{3}$ fragments deposited on antigen-presenting cells. J Leukoc Biol, 2001. 69(1): p. 6974 .

84. Cooper, P.D. and M. Carter, The anti-melanoma activity of inulin in mice. Mol Immunol, 1986. 23(8): p. $903-8$.

85. Yamashita, A., H. Hashimoto, K. Fujita, M. Okada, S. Mori, and S. Kitahata, Reverse reaction of Aspergillus niger APC-9319 alpha-galactosidase in a supersaturated substrate solution: production of alpha-linked galactooligosaccharide (alpha-GOS). Biosci Biotechnol Biochem, 2005. 69(7): p. 1381-8.

86. Kan, T., Y. Kobayashi, and K. Matsumoto, Character of galacto-oligosaccharides and application for food. New Food Industry, 1989. 31: p. 25-30.

87. Shoaf, K., G.L. Mulvey, G.D. Armstrong, and R.W. Hutkins, Prebiotic galactooligosaccharides reduce adherence of Enteropathogenic Escherichia coli to tissue culture cells. Infect Immun, 2006.

88. Holma, R., P. Juvonen, M.Z. Asmawi, H. Vapaatalo, and R. Korpela, Galacto-oligosaccharides stimulate the growth of bifidobacteria but fail to attenuate inflammation in experimental colitis in rats. Scand J Gastroenterol, 2002. 37(9): p. 1042-7.

89. Sonoyama, K., H. Watanabe, J. Watanabe, N. Yamaguchi, A. Yamashita, H. Hashimoto, E. Kishino, K. Fujita, M. Okada, S. Mori, S. Kitahata, and J. Kawabata, Allergic airway eosinophilia is suppressed in ovalbumin-sensitized Brown Norway rats fed raffinose and alpha-linked galactooligosaccharide. J Nutr, 2005. 135(3): p. 538-43.

9o. Abe, C., K. Fujita, E. Kikuchi, S. Hirano, H. Kuboki, A. Yamashita, H. Hashimoto, S. Mori, and M. Okada, Effects of alpha-linked galactooligosaccharide on adjuvant-induced arthritis in Wistar rats and type II collagen-induced arthritis in DBA/1 J mice. Int J Tissue React, 2004. 26(3-4): p. 65-73. 
91. Watanabe, H., K. Sonoyama, J. Watanabe, N. Yamaguchi, H. Kikuchi, T. Nagura, T. Aritsuka, K. Fukumoto, and T. Kasai, Reduction of allergic airway eosinophilia by dietary raffinose in Brown Norway rats. Br J Nutr, 2004. 92(2): p. 247-55.

92. Nagura, T., S. Hachimura, M. Hashiguchi, Y. Ueda, T. Kanno, H. Kikuchi, K. Sayama, and S. Kaminogawa, Suppressive effect of dietary raffinose on T-helper 2 cell-mediated immunity. Br J Nutr, 2002. 88(4): p. 421-6.

93. Boehm, G., S. Fanaro, J. Jelinek, B. Stahl, and A. Marini, Prebiotic concept for infant nutrition. Acta Paediatr Suppl, 2003. 91(441): p. 64-7.

94. Fanaro, S., J. Jelinek, B. Stahl, G. Boehm, R. Kock, and V. Vigi, Acidic oligosaccharides from pectin hydrolysate as new component for infant formulae: effect on intestinal flora, stool characteristics, and pH. J Pediatr Gastroenterol Nutr, 2005. 41(2): p. 186-90.

95. Tsuji, R.F., G.P. Geba, Y. Wang, K. Kawamoto, L.A. Matis, and P.W. Askenase, Required early complement activation in contact sensitivity with generation of local $C_{5}$-dependent chemotactic activity, and late T cell interferon gamma: a possible initiating role of B cells. J Exp Med, 1997. 186(7): p. 1015-26.

96. Szczepanik, M., M. Akahira-Azuma, K. Bryniarski, R.F. Tsuji, I. Kawikova, W. Ptak, C. Kiener, R.A. Campos, and P.W. Askenase, B-1 B cells mediate required early $T$ cell recruitment to elicit proteininduced delayed-type hypersensitivity. J Immunol, 2003. 171(11): p. 6225-35.

97. Moro, G., S. Arslanoglu, B. Stahl, J. Jelinek, U. Wahn, and G. Boehm, A mixture of prebiotic oligosaccharides reduces the incidence of atopic dermatitis during the first six months of age. Arch Dis Child, 2006. 91(10): p. 814-9.

98. Bjorksten, B., E. Sepp, K. Julge, T. Voor, and M. Mikelsaar, Allergy development and the intestinal microflora during the first year of life. J Allergy Clin Immunol, 2001. 108(4): p. 516-20.

99. Bakker-Zierikzee, A.M., E.A. Tol, H. Kroes, M.S. Alles, F.J. Kok, and J.G. Bindels, Faecal SlgA secretion in infants fed on pre- or probiotic infant formula. Pediatr Allergy Immunol, 2006. 17(2): p. 134-40.

100. Mullie, C., A. Yazourh, H. Thibault, M.F. Odou, E. Singer, N. Kalach, O. Kremp, and M.B. Romond, Increased poliovirus-specific intestinal antibody response coincides with promotion of Bifidobacterium longum-infantis and Bifidobacterium breve in infants: a randomized, double-blind, placebo-controlled trial. Pediatr Res, 2004. 56(5): p. 791-5.

101. Corkins, M.R., Prebiotics: do they really change infant intestinal immunity? J Pediatr Gastroenterol Nutr, 2005. 40(3): p. 384-5.

102. Manners, D.J., A.J. Masson, J.C. Patterson, H. Bjorndal, and B. Lindberg, The structure of a beta-(1-6)-D-glucan from yeast cell walls. Biochem J, 1973. 135(1): p. 31-6.

103. Manners, D.J., A.J. Masson, and J.C. Patterson, The structure of a beta-(1 leads to 3)-D-glucan from yeast cell walls. Biochem J, 1973. 135(1): p. 19-30.

104. Ensley, H.E., B. Tobias, H.A. Pretus, R.B. McNamee, E.L. Jones, I.W. Browder, and D.L. Williams, NMR spectral analysis of a water-insoluble (1-->3)-beta-D-glucan isolated from Saccharomyces cerevisiae. Carbohydr Res, 1994. 258: p. 307-11.

105. Wood, P.J., J. Weisz, and B.A. Blackwell, Molecular characterization of cereal beta-D-glucans. Structural analysis of oat beta-D-glucan and rapid structural evaluation of beta-D-glucans from different sources by high-performance liquid chromatography of oligosaccharides released by lichenase. Cereal Chem, 1991. 68(1): p. 31-39.

106. Kim, S.Y., H.J. Song, Y.Y. Lee, K.H. Cho, and Y.K. Roh, Biomedical issues of dietary fiber beta-glucan. J Korean Med Sci, 2006. 21(5): p. 781-9.

107. Zekovic, D.B., S. Kwiatkowski, M.M. Vrvic, D. Jakovljevic, and C.A. Moran, Natural and modified (1->3)-beta-D-glucans in health promotion and disease alleviation. Crit Rev Biotechnol, 2005. 25(4): p. 205-30. 
108. Campa-Cordova, A.I., N.Y. Hernandez-Saavedra, R. De Philippis, and F. Ascencio, Generation of superoxide anion and SOD activity in haemocytes and muscle of American white shrimp (Litopenaeus vannamei) as a response to beta-glucan and sulphated polysaccharide. Fish Shellfish Immunol, 2002. 12(4): p. 353-66.

109. Chang, C.F., M.S. Su, H.Y. Chen, and I.C. Liao, Dietary beta-1,3-glucan effectively improves immunity and survival of Penaeus monodon challenged with white spot syndrome virus. Fish Shellfish Immunol, 2003. 15(4): p. 297-310.

110. Bagni, M., N. Romano, M.G. Finoia, L. Abelli, G. Scapigliati, P.G. Tiscar, M. Sarti, and G. Marino, Short- and long-term effects of a dietary yeast beta-glucan (Macrogard) and alginic acid (Ergosan) preparation on immune response in sea bass (Dicentrarchus labrax). Fish Shellfish Immunol, 2005. 18(4): p. 311-25.

111. Kumari, J. and P.K. Sahoo, Dietary beta-1,3 glucan potentiates innate immunity and disease resistance of Asian catfish, Clarias batrachus (L.). J Fish Dis, 2006. 29(2): p. 95-101.

112. Lowry, V.K., M.B. Farnell, P.J. Ferro, C.L. Swaggerty, A. Bahl, and M.H. Kogut, Purified beta-glucan as an abiotic feed additive up-regulates the innate immune response in immature chickens against Salmonella enterica serovar Enteritidis. Int J Food Microbiol, 2005. 98(3): p. 309-18.

113. Li, J., J. Xing, D. Li, X. Wang, L. Zhao, S. Lv, and D. Huang, Effects of beta-glucan extracted from Saccharomyces cerevisiae on humoral and cellular immunity in weaned piglets. Arch Anim Nutr, 2005. 59(5): p. 303-12.

114. Jung, K., Y. Ha, S.K. Ha, D.U. Han, D.W. Kim, W.K. Moon, and C. Chae, Antiviral effect of Saccharomyces cerevisiae beta-glucan to swine influenza virus by increased production of interferongamma and nitric oxide. J Vet Med B Infect Dis Vet Public Health, 2004. 51(2): p. $72-6$.

115. Davis, J.M., E.A. Murphy, A.S. Brown, M.D. Carmichael, A. Ghaffar, and E.P. Mayer, Effects of oat beta-glucan on innate immunity and infection after exercise stress. Med Sci Sports Exerc, 2004. 36(8): p. 1321-7.

116. Suzuki, I., K. Hashimoto, N. Ohno, H. Tanaka, and T. Yadomae, Immunomodulation by orally administered beta-glucan in mice. Int J Immunopharmacol, 1989. 11(7): p. 761-9.

117. Tsukada, C., H. Yokoyama, C. Miyaji, Y. Ishimoto, H. Kawamura, and T. Abo, Immunopotentiation of intraepithelial lymphocytes in the intestine by oral administrations of beta-glucan. Cell Immunol, 2003. 221(1): p. 1-5.

118. Yun, C.H., A. Estrada, A. Van Kessel, B.C. Park, and B. Laarveld, Beta-glucan, extracted from oat, enhances disease resistance against bacterial and parasitic infections. FEMS Immunol Med Microbiol, 2003. 35(1): p. 67-75.

119. Lehne, G., B. Haneberg, P. Gaustad, P.W. Johansen, H. Preus, and T.G. Abrahamsen, Oral administration of a new soluble branched beta-1,3-D-glucan is well tolerated and can lead to increased salivary concentrations of immunoglobulin A in healthy volunteers. Clin Exp Immunol, 2006. 143(1): p. 65-9.

120. Kirmaz, C., P. Bayrak, O. Yilmaz, and H. Yuksel, Effects of glucan treatment on the Th1/Th2 balance in patients with allergic rhinitis: a double-blind placebo-controlled study. Eur Cytokine Netw, 2005. 16(2): p. 128-34.

121. Poutsiaka, D.D., M. Mengozzi, E. Vannier, B. Sinha, and C.A. Dinarello, Cross-linking of the betaglucan receptor on human monocytes results in interleukin-1 receptor antagonist but not interleukin1 production. Blood, 1993. 82(12): p. 3695-700.

122. Hunter, K.W., Jr., S. DuPre, and D. Redelman, Microparticulate beta-glucan upregulates the expression of $B_{7.1}, B_{7} .2, B 7-H_{1}$, but not $B 7-D C$ on cultured murine peritoneal macrophages. Immunol Lett, 2004. 93(1): p. 71-8. 
123. Luhm, J., U. Langenkamp, J. Hensel, C. Frohn, J.M. Brand, H. Hennig, L. Rink, P. Koritke, N. Wittkopf, D.L. Williams, and A. Mueller, Beta-(1-->3)-D-glucan modulates DNA binding of nuclear factors kappaB, AT and IL-6 leading to an anti-inflammatory shift of the IL-1beta/IL-1 receptor antagonist ratio. BMC Immunol, 2006. 7: p. 5.

124. Engstad, C.S., R.E. Engstad, J.O. Olsen, and B. Osterud, The effect of soluble beta-1,3-glucan and lipopolysaccharide on cytokine production and coagulation activation in whole blood. Int Immunopharmacol, 2002. 2(11): p. 1585-97.

125. Lee, J.N., D.Y. Lee, I.H. Ji, G.E. Kim, H.N. Kim, J. Sohn, S. Kim, and C.W. Kim, Purification of soluble beta-glucan with immune-enhancing activity from the cell wall of yeast. Biosci Biotechnol Biochem, 2001. 65(4): p. 837-41.

126. Berner, M.D., M.E. Sura, B.N. Alves, and K.W. Hunter, Jr., IFN-gamma primes macrophages for enhanced TNF-alpha expression in response to stimulatory and non-stimulatory amounts of microparticulate beta-glucan. Immunol Lett, 2005. 98(1): p. 115-22.

127. Sakurai, T., N. Ohno, and T. Yadomae, Effects of fungal beta-glucan and interferon-gamma on the secretory functions of murine alveolar macrophages. J Leukoc Biol, 1996. 6o(1): p. 118-24.

128. Adachi, Y., M. Okazaki, N. Ohno, and T. Yadomae, Enhancement of cytokine production by macrophages stimulated with (1-->3)-beta-D-glucan, grifolan (GRN), isolated from Grifola frondosa. Biol Pharm Bull, 1994. 17(12): p. 1554-60.

129. Estrada, A., C.H. Yun, A. Van Kessel, B. Li, S. Hauta, and B. Laarveld, Immunomodulatory activities of oat beta-glucan in vitro and in vivo. Microbiol Immunol, 1997. 41(12): p. 991-8.

130. Lowe, E., P. Rice, T. Ha, C. Li, J. Kelley, H. Ensley, J. Lopez-Perez, J. Kalbfleisch, D. Lowman, P. Margl, W. Browder, and D. Williams, A (1-->3)-beta-D-linked heptasaccharide is the unit ligand for glucan pattern recognition receptors on human monocytes. Microbes Infect, 2001. 3(10): p. 789-97.

131. Harler, M.B. and J. Reichner, Increased neutrophil motility by beta-glucan in the absence of chemoattractant. Shock, 2001. 16(6): p. 419-24.

132. Duan, X., M. Ackerly, E. Vivier, and P. Anderson, Evidence for involvement of beta-glucan-binding cell surface lectins in human natural killer cell function. Cell Immunol, 1994. 157(2): p. 393-402.

133. Di Renzo, L., E. Yefenof, and E. Klein, The function of human NK cells is enhanced by beta-glucan, a ligand of $C_{3}$ (CD11b/CD18). Eur J Immunol, 1991. 21(7): p. 1755-8.

134. Brown, G.D., P.R. Taylor, D.M. Reid, J.A. Willment, D.L. Williams, L. Martinez-Pomares, S.Y. Wong, and S. Gordon, Dectin-1 is a major beta-glucan receptor on macrophages. J Exp Med, 2002. 196(3): p. 407-12.

135. Willment, J.A., A.S. Marshall, D.M. Reid, D.L. Williams, S.Y. Wong, S. Gordon, and G.D. Brown, The human beta-glucan receptor is widely expressed and functionally equivalent to murine Dectin-1 on primary cells. Eur J Immunol, 2005. 35(5): p. 1539-47.

136. Rogers, N.C., E.C. Slack, A.D. Edwards, M.A. Nolte, O. Schulz, E. Schweighoffer, D.L. Williams, S. Gordon, V.L. Tybulewicz, G.D. Brown, and C. Reis e Sousa, Syk-dependent cytokine induction by Dectin-1 reveals a novel pattern recognition pathway for $C$ type lectins. Immunity, 2005. 22(4): p. 50717.

137. Omarsdottir, S., E.S. Olafsdottir, and J. Freysdottir, Immunomodulating effects of lichen-derived polysaccharides on monocyte-derived dendritic cells. Int Immunopharmacol, 2006. 6(11): p. 1642-50.

138. Kikuchi, T., N. Ohno, and T. Ohno, Maturation of dendritic cells induced by Candida beta-D-glucan. Int Immunopharmacol, 2002. 2(10): p. 1503-8.

139. Yun, C.H., A. Estrada, A. Van Kessel, A.A. Gajadhar, M.J. Redmond, and B. Laarveld, beta-(1-->3, 1-$>4$ ) oat glucan enhances resistance to Eimeria vermiformis infection in immunosuppressed mice. Int J Parasitol, 1997. 27(3): p. 329-37. 
140. Hanaue, H., Y. Tokuda, T. Machimura, A. Kamijoh, Y. Kondo, K. Ogoshi, H. Makuuchi, H. Nakasaki, T. Tajima, T. Mitomi, and et al., Effects of oral lentinan on T-cell subsets in peripheral venous blood. Clin Ther, 1989. 11(5): p. 614-22.

141. Ng, M.L. and A.T. Yap, Inhibition of human colon carcinoma development by lentinan from shiitake mushrooms (Lentinus edodes). J Altern Complement Med, 2002. 8(5): p. 581-9.

142. Cheung, N.K., S. Modak, A. Vickers, and B. Knuckles, Orally administered beta-glucans enhance antitumor effects of monoclonal antibodies. Cancer Immunol Immunother, 2002. 51(10): p. 557-64.

143. Modak, S., G. Koehne, A. Vickers, R.J. O'Reilly, and N.K. Cheung, Rituximab therapy of lymphoma is enhanced by orally administered (1-->3),(1-->4)-D-beta-glucan. Leuk Res, 2005. 29(6): p. 679-83.

144. Drochner, W., A. Kerler, and B. Zacharias, Pectin in pig nutrition, a comparative review. J Anim Physiol Anim Nutr (Berl), 2004. 88(11-12): p. 367-80.

145. Platt, D. and A. Raz, Modulation of the lung colonization of B16-F1 melanoma cells by citrus pectin. J Natl Cancer Inst, 1992. 84: p. 438-442.

146. Dongowski, G., A. Lorenz, and J. Proll, The degree of methylation influences the degradation of pectin in the intestinal tract of rats and in vitro. J Nutr, 2002. 132(7): p. 1935-44.

147. lacomini, M., R.V. Serrato, G.L. Sassaki, L. Lopes, D.F. Buchi, and P.A. Gorin, Isolation and partial characterization of a pectic polysaccharide from the fruit pulp of Spondias cytherea and its effect on peritoneal macrophage activation. Fitoterapia, 2005. 76(7-8): p. 676-83.

148. Dourado, F., P. Madureira, V. Carvalho, R. Coelho, M.A. Coimbra, M. Vilanova, M. Mota, and F.M. Gama, Purification, structure and immunobiological activity of an arabinan-rich pectic polysaccharide from the cell walls of Prunus dulcis seeds. Carbohydr Res, 2004. 339(15): p. 2555-66.

149. Chen, C.H., M.T. Sheu, T.F. Chen, Y.C. Wang, W.C. Hou, D.Z. Liu, T.C. Chung, and Y.C. Liang, Suppression of endotoxin-induced proinflammatory responses by citrus pectin through blocking LPS signaling pathways. Biochem Pharmacol, 2006. 72(8): p. 1001-9.

150. Guggenbichler, J.P., A. De Bettignies-Dutz, P. Meissner, S. Schellmoser, and J. Jurenitsch, Acidic oligosaccharides from natural sources block adherence of Escherichia coli on uroepithelial cells. Pharm Pharmacol Lett, 1997. 7(1): p. 35-38.

151. Kastner, U., S. Glasl, B. Follrich, J.P. Guggenbichler, and J. Jurenitsch, [Acid oligosaccharides as the active principle of aqueous carrot extracts for prevention and therapy of gastrointestinal infections]. Wien Med Wochenschr, 2002. 152(15-16): p. 379-81.

152. Olano-Martin, E., M.R. Williams, G.R. Gibson, and R.A. Rastall, Pectins and pectic-oligosaccharides inhibit Escherichia coli $\mathrm{O}_{157}: \mathrm{H}_{7}$ Shiga toxin as directed towards the human colonic cell line $\mathrm{HT}_{29}$. FEMS Microbiol Lett, 2003. 218(1): p. 101-5.

153. Lee, J.C., S.C. Pak, S.H. Lee, C.S. Na, S.C. Lim, C.H. Song, Y.H. Bai, and C.H. Jang, Asian pear pectin administration during presensitization inhibits allergic response to ovalbumin in BALB/C mice. J Altern Complement Med, 2004. 10(3): p. 527-34.

154. Lim, B.O., K. Yamada, M. Nonaka, Y. Kuramoto, P. Hung, and M. Sugano, Dietary fibers modulate indices of intestinal immune function in rats. J Nutr, 1997. 127(5): p. 663-7.

155. Lim, B.O., S.H. Lee, D.K. Park, and R.W. Chove, Effect of dietary pectin on the production of immunoglobulins and cytokines by mesenteric lymph node lymphocytes in mouse colitis induced with dextran sulfate sodium. Biosci Biotechnol Biochem, 2003. 67(8): p. 1706-12.

156. Nangia-Makker, P., V. Hogan, Y. Honjo, S. Baccarini, L. Tait, R. Bresalier, and A. Raz, Inhibition of human cancer cell growth and metastasis in nude mice by oral intake of modified citrus pectin. J Natl Cancer Inst, 2002. 94(24): p. 1854-62.

157. Pienta, K.J., H. Naik, A. Akhtar, K. Yamazaki, T.S. Replogle, J. Lehr, T.L. Donat, L. Tait, V. Hogan, and A. Raz, Inhibition of spontaneous metastasis in a rat prostate cancer model by oral administration of modified citrus pectin. J Natl Cancer Inst, 1995. 87(5): p. 348-53. 
158. Inohara, H. and A. Raz, Effects of natural complex carbohydrate (citrus pectin) on murine melanoma cell properties related to galectin-3 functions. Glycoconj J, 1994. 11(6): p. 527-32.

159. Guess, B.W., M.C. Scholz, S.B. Strum, R.Y. Lam, H.J. Johnson, and R.I. Jennrich, Modified citrus pectin (MCP) increases the prostate-specific antigen doubling time in men with prostate cancer: a phase II pilot study. Prostate Cancer Prostatic Dis, 2003. 6(4): p. 301-4.

160. Vos, A.P., M. Haarman, J.W.H. van Ginkel, J. Knol, J. Garssen, B. Stahl, G. Boehm, and L. M'Rabet, Dietary supplementation of neutral and acidic oligosaccharides enhances Th1-dependent vaccination responses in mice. Pediatr Allergy Immunol, In Press.

161. Sakurai, M.H., T. Matsumoto, H. Kiyohara, and H. Yamada, B-cell proliferation activity of pectic polysaccharide from a medicinal herb, the roots of Bupleurum falcatum $L$. and its structural requirement. Immunology, 1999. 97(3): p. 540-7.

162. Kiyohara, H., T. Matsumoto, N. Takemoto, H. Kawamura, Y. Komatsu, and H. Yamada, Effect of oral administration of a pectic polysaccharide fraction from a kampo (Japanese herbal) medicine "juzentaiho-to" on antibody response of mice. Planta Med, 1995. 61(5): p. 429-34.

163. Nergard, C.S., T. Matsumoto, M. Inngjerdingen, K. Inngjerdingen, S. Hokputsa, S.E. Harding, T.E. Michaelsen, D. Diallo, H. Kiyohara, B.S. Paulsen, and H. Yamada, Structural and immunological studies of a pectin and a pectic arabinogalactan from Vernonia kotschyana Sch. Bip. ex Walp. (Asteraceae). Carbohydr Res, 2005. 340(1): p. 115-30.

164. Michaelsen, T.E., A. Gilje, A.B. Samuelsen, K. Hogasen, and B.S. Paulsen, Interaction between human complement and a pectin type polysaccharide fraction, PMII, from the leaves of Plantago major $\mathrm{L}$. Scand J Immunol, 2000. 52(5): p. 483-90.

165. Popov, S.V., V.V. Golovchenko, R.G. Ovodova, V.V. Smirnov, D.S. Khramova, G.Y. Popova, and Y.S. Ovodov, Characterisation of the oral adjuvant effect of lemnan, a pectic polysaccharide of Lemna minor L. Vaccine, 2006. 24(26): p. 5413-9.

166. Popov, S.V., G.Y. Popova, S.Y. Nikolaeva, V.V. Golovchenko, and R.G. Ovodova, Immunostimulating activity of pectic polysaccharide from Bergenia crassifolia (L.) Fritsch. Phytother Res, 2005. 19(12): p. 1052-6.

167. Topping, D.L. and P.M. Clifton, Short-chain fatty acids and human colonic function: roles of resistant starch and nonstarch polysaccharides. Physiol Rev, 2001. 81(3): p. 1031-64.

168. Moreau, N.M., L.J. Martin, C.S. Toquet, C.L. Laboisse, P.G. Nguyen, B.S. Siliart, H.J. Dumon, and M.M. Champ, Restoration of the integrity of rat caeco-colonic mucosa by resistant starch, but not by fructo-oligosaccharides, in dextran sulfate sodium-induced experimental colitis. Br J Nutr, 2003. 9o(1): p. $75-85$.

169. Moreau, N.M., M.M. Champ, S.M. Goupry, B.J. Le Bizec, M. Krempf, P.G. Nguyen, H.J. Dumon, and L.J. Martin, Resistant starch modulates in vivo colonic butyrate uptake and its oxidation in rats with dextran sulfate sodium-induced colitis. J Nutr, 2004. 134(3): p. 493-500.

170. Jacobasch, G., D. Schmiedl, M. Kruschewski, and K. Schmehl, Dietary resistant starch and chronic inflammatory bowel diseases. Int J Colorectal Dis, 1999. 14(4-5): p. 201-11.

171. Murosaki, S., K. Muroyama, Y. Yamamoto, H. Kusaka, T. Liu, and Y. Yoshikai, Immunopotentiating activity of nigerooligosaccharides for the $T$ helper 1-like immune response in mice. Biosci Biotechnol Biochem, 1999. 63(2): p. 373-8.

172. Murosak, S., K. Muroyama, Y. Yamamoto, T. Liu, and Y. Yoshikai, Nigerooligosaccharides augments natural killer activity of hepatic mononuclear cells in mice. Int Immunopharmacol, 2002. 2(1): p. 1519 .

173. Hirose, Y., S. Murosaki, Y. Yamamoto, H. Ikematsu, and K. Nomoto, Nigerooligosaccharides augments mitogen-induced proliferation and suppresses activation-induced apoptosis of human peripheral blood mononuclear cells. Immunopharmacol Immunotoxicol, 2004. 26(3): p. 387-99.

174. Mizubuchi, H., T. Yajima, N. Aoi, T. Tomita, and Y. Yoshikai, Isomalto-oligosaccharides polarize Th1like responses in intestinal and systemic immunity in mice. J Nutr, 2005. 135(12): p. 2857-61. 
175. Kelly, G.S., Larch arabinogalactan: clinical relevance of a novel immune-enhancing polysaccharide. Altern Med Rev, 1999. 4(2): p. 96-103.

176. Haver, J. and F.A. Anderer, Mechanism of stimulation of human natural killer cytotoxicity by arabinogalactan from Larix occidentalis. Cancer Immunol Immunother, 1993. 36(4): p. 237-44.

177. Naito, Y., T. Takagi, K. Katada, K. Uchiyama, M. Kuroda, S. Kokura, H. Ichikawa, J. Watabe, N. Yoshida, T. Okanoue, and T. Yoshikawa, Partially hydrolyzed guar gum down-regulates colonic inflammatory response in dextran sulfate sodium-induced colitis in mice. J Nutr Biochem, 2006. 17(6): p. 402-9.

178. Yamada, K., Y. Tokunaga, A. Ikeda, K. Ohkura, S. Kaku-Ohkura, S. Mamiya, B.O. Lim, and H. Tachibana, Effect of dietary fiber on the lipid metabolism and immune function of aged SpragueDawley rats. Biosci Biotechnol Biochem, 2003. 67(2): p. 429-33.

179. Engfer, M.B., B. Stahl, B. Finke, G. Sawatzki, and H. Daniel, Human milk oligosaccharides are resistant to enzymatic hydrolysis in the upper gastrointestinal tract. Am J Clin Nutr, 2000. 71(6): p. 1589-96.

180. Ward, R.E., M. Ninonuevo, D.A. Mills, C.B. Lebrilla, and J.B. German, In vitro fermentation of breast milk oligosaccharides by Bifidobacterium infantis and Lactobacillus gasseri. Appl Environ Microbiol, 2006. 72(6): p. 4497-9.

181. Cummings, J.H. and G.T. Macfarlane, Gastrointestinal effects of prebiotics. Br J Nutr, 2002. 87 Suppl 2: p. S145-51.

182. Tuohy, K.M., S. Kolida, A.M. Lustenberger, and G.R. Gibson, The prebiotic effects of biscuits containing partially hydrolysed guar gum and fructo-oligosaccharides--a human volunteer study. Br J Nutr, 2001. 86(3): p. 341-8.

183. Bouhnik, Y., L. Raskine, G. Simoneau, D. Paineau, and F. Bornet, The capacity of short-chain fructooligosaccharides to stimulate faecal bifidobacteria: a dose-response relationship study in healthy humans. Nutr J, 2006. 5: p. 8.

184. Langlands, S.J., M.J. Hopkins, N. Coleman, and J.H. Cummings, Prebiotic carbohydrates modify the mucosa associated microflora of the human large bowel. Gut, 2004. 53(11): p. 1610-6.

185. Kapiki, A., C. Costalos, C. Oikonomidou, A. Triantafyllidou, E. Loukatou, and V. Pertrohilou, The effect of a fructo-oligosaccharide supplemented formula on gut flora of preterm infants. Early Hum Dev, 2006.

186. Euler, A.R., D.K. Mitchell, R. Kline, and L.K. Pickering, Prebiotic effect of fructo-oligosaccharide supplemented term infant formula at two concentrations compared with unsupplemented formula and human milk. J Pediatr Gastroenterol Nutr, 2005. 40(2): p. 157-64.

187. Brunser, O., G. Figueroa, M. Gotteland, E. Haschke-Becher, C. Magliola, F. Rochat, S. Cruchet, R. Palframan, G. Gibson, F. Chauffard, and F. Haschke, Effects of probiotic or prebiotic supplemented milk formulas on fecal microbiota composition of infants. Asia Pac J Clin Nutr, 2006. 15(3): p. 368-76.

188. Brunser, O., M. Gotteland, S. Cruchet, G. Figueroa, D. Garrido, and P. Steenhout, Effect of a milk formula with prebiotics on the intestinal microbiota of infants after an antibiotic treatment. Pediatr Res, 2006. 59(3): p. 451-6.

189. Roberfroid, M.B., Introducing inulin-type fructans. Br J Nutr, 2005. 93 Suppl 1: p. S13-25.

190. Rossi, M., C. Corradini, A. Amaretti, M. Nicolini, A. Pompei, S. Zanoni, and D. Matteuzzi, Fermentation of fructooligosaccharides and inulin by bifidobacteria: a comparative study of pure and fecal cultures. Appl Environ Microbiol, 2005. 71(10): p. 6150-8.

191. Mountzouris, K.C., C. Balaskas, F. Fava, K.M. Tuohy, G.R. Gibson, and K. Fegeros, Profiling of composition and metabolic activities of the colonic microflora of growing pigs fed diets supplemented with prebiotic oligosaccharides. Anaerobe, 2006. 
192. Howard, M.D., D.T. Gordon, K.A. Garleb, and M.S. Kerley, Dietary fructooligosaccharide, xylooligosaccharide and gum arabic have variable effects on cecal and colonic microbiota and epithelial cell proliferation in mice and rats. J Nutr, 1995. 125(10): p. 2604-9.

193. Ito, M., Y. Deguchi, K. Matsumoto, M. Kimura, N. Onodera, and T. Yajima, Influence of galactooligosaccharides on the human fecal microflora. J Nutr Sci Vitaminol (Tokyo), 1993. 39(6): p. 635-40.

194. Ito, M., M. Kimura, Y. Deguchi, A. Miyamori-Watabe, T. Yajima, and T. Kan, Effects of transgalactosylated disaccharides on the human intestinal microflora and their metabolism. J Nutr Sci Vitaminol (Tokyo), 1993. 39(3): p. 279-88.

195. Bouhnik, Y., B. Flourie, L. D'Agay-Abensour, P. Pochart, G. Gramet, M. Durand, and J.C. Rambaud, Administration of transgalacto-oligosaccharides increases fecal bifidobacteria and modifies colonic fermentation metabolism in healthy humans. J Nutr, 1997. 127(3): p. 444-8.

196. Alles, M.S., R. Hartemink, S. Meyboom, J.L. Harryvan, K.M. Van Laere, F.M. Nagengast, and J.G. Hautvast, Effect of transgalactooligosaccharides on the composition of the human intestinal microflora and on putative risk markers for colon cancer. Am J Clin Nutr, 1999. 69(5): p. 980-91.

197. Rowland, I.R. and R. Tanaka, The effects of transgalactosylated oligosaccharides on gut flora metabolism in rats associated with a human faecal microflora. J Appl Bacteriol, 1993. 74(6): p. 667-74.

198. Kikuchi, H., C. Andrieux, M. Riottot, M. Bensaada, F. Popot, P. Beaumatin, and O. Szylit, Effect of two levels of transgalactosylated oligosaccharide intake in rats associated with human faecal microflora on bacterial glycolytic activity, end-products of fermentation and bacterial steroid transformation. J Appl Bacteriol, 1996. 8o(4): p. 439-46.

199. Tzortzis, G., A.K. Goulas, J.M. Gee, and G.R. Gibson, A novel galactooligosaccharide mixture increases the bifidobacterial population numbers in a continuous in vitro fermentation system and in the proximal colonic contents of pigs in vivo. J Nutr, 2005. 135(7): p. 1726-31.

200. Smiricky-Tjardes, M.R., E.A. Flickinger, C.M. Grieshop, L.L. Bauer, M.R. Murphy, and G.C. Fahey, Jr., In vitro fermentation characteristics of selected oligosaccharides by swine fecal microflora. J Anim Sci, 2003. 81(10): p. 2505-14.

201. Tzortzis, G., A.K. Goulas, M.L. Baillon, G.R. Gibson, and R.A. Rastall, In vitro evaluation of the fermentation properties of galactooligosaccharides synthesised by alpha-galactosidase from Lactobacillus reuteri. Appl Microbiol Biotechnol, 2004. 64(1): p. 106-11.

202. Moro, G., I. Minoli, M. Mosca, S. Fanaro, J. Jelinek, B. Stahl, and G. Boehm, Dosage-related bifidogenic effects of galacto- and fructooligosaccharides in formula-fed term infants. J Pediatr Gastroenterol Nutr, 2002. 34(3): p. 291-5.

203. Moro, G.E., F. Mosca, V. Miniello, S. Fanaro, J. Jelinek, B. Stahl, and G. Boehm, Effects of a new mixture of prebiotics on faecal flora and stools in term infants. Acta Paediatr Suppl, 2003. 91(441): p. 77-9.

204. Moro, G.E., B. Stahl, S. Fanaro, J. Jelinek, G. Boehm, and G.V. Coppa, Dietary prebiotic oligosaccharides are detectable in the faeces of formula-fed infants. Acta Paediatr Suppl, 2005. 94(449): p. 27-30.

205. Bakker-Zierikzee, A.M., M.S. Alles, J. Knol, F.J. Kok, J.J. Tolboom, and J.G. Bindels, Effects of infant formula containing a mixture of galacto- and fructo-oligosaccharides or viable Bifidobacterium animalis on the intestinal microflora during the first 4 months of life. Br J Nutr, 2005. 94(5): p. 783-90.

206. Knol, J., P. Scholtens, C. Kafka, J. Steenbakkers, S. Gro, K. Helm, M. Klarczyk, H. Schopfer, H.M. Bockler, and J. Wells, Colon microflora in infants fed formula with galacto- and fructooligosaccharides: more like breast-fed infants. J Pediatr Gastroenterol Nutr, 2005. 40(1): p. 36-42.

207. Scholtens, P.A., M.S. Alles, J.G. Bindels, E.G. van der Linde, J.J. Tolboom, and J. Knol, Bifidogenic Effects of Solid Weaning Foods With Added Prebiotic Oligosaccharides: A Randomised Controlled Clinical Trial. J Pediatr Gastroenterol Nutr, 2006. 42(5): p. 553-559. 
208. Hsu, C.K., J.W. Liao, Y.C. Chung, C.P. Hsieh, and Y.C. Chan, Xylooligosaccharides and fructooligosaccharides affect the intestinal microbiota and precancerous colonic lesion development in rats. J Nutr, 2004. 134(6): p. 1523-8.

209. Olano-Martin, E., K.C. Mountzouris, G.R. Gibson, and R.A. Rastall, In vitro fermentability of dextran, oligodextran and maltodextrin by human gut bacteria. Br J Nutr, 2000. 83(3): p. 247-55.

210. Rabelo, M.C., T.L. Honorato, L.R. Goncalves, G.A. Pinto, and S. Rodrigues, Enzymatic synthesis of prebiotic oligosaccharides. Appl Biochem Biotechnol, 2006. 133(1): p. 31-40.

211. Sanz, M.L., G.L. Cote, G.R. Gibson, and R.A. Rastall, Prebiotic properties of alternansucrase maltoseacceptor oligosaccharides. J Agric Food Chem, 2005. 53(15): p. 5911-6.

212. Sanz, M.L., G.R. Gibson, and R.A. Rastall, Influence of disaccharide structure on prebiotic selectivity in vitro. J Agric Food Chem, 2005. 53(13): p. 5192-9.

213. Sanz, M.L., G.L. Cote, G.R. Gibson, and R.A. Rastall, Selective fermentation of gentiobiose-derived oligosaccharides by human gut bacteria and influence of molecular weight. FEMS Microbiol Ecol, 2006. 56(3): p. 383-8.

214. Sanz, M.L., N. Polemis, V. Morales, N. Corzo, A. Drakoularakou, G.R. Gibson, and R.A. Rastall, In vitro investigation into the potential prebiotic activity of honey oligosaccharides. J Agric Food Chem, 2005. 53(8): p. 2914-21.

215. Chung, C.H. and D.F. Day, Glucooligosaccharides from Leuconostoc mesenteroides B-742 (ATCC 13146): a potential prebiotic. J Ind Microbiol Biotechnol, 2002. 29(4): p. 196-9.

216. Al-Tamimi, M.A., R.J. Palframan, J.M. Cooper, G.R. Gibson, and R.A. Rastall, In vitro fermentation of sugar beet arabinan and arabino-oligosaccharides by the human gut microflora. J Appl Microbiol, 2006. 100(2): p. 407-14.

217. Snart, J., R. Bibiloni, T. Grayson, C. Lay, H. Zhang, G.E. Allison, J.K. Laverdiere, F. Temelli, T. Vasanthan, R. Bell, and G.W. Tannock, Supplementation of the diet with high-viscosity beta-glucan results in enrichment for lactobacilli in the rat cecum. Appl Environ Microbiol, 2006. 72(3): p. 1925-31.

218. Dongowski, G., M. Huth, E. Gebhardt, and W. Flamme, Dietary fiber-rich barley products beneficially affect the intestinal tract of rats. J Nutr, 2002. 132(12): p. 3704-14.

219. Malkki, Y., Oat fiber. Production, composition, physicochemical properties, physiological effects, safety, and food applications, in Handbook of dietary fiber, S.S. Cho and M.L. Dreher, Editors. 2001, Marcel Dekker: New York.

220. Lund, E.K. and I.T. Johnson, Fermentable carbohydrate reaching the colon after ingestion of oats in humans. J Nutr, 1991. 121(3): p. 311-7.

221. Vargo, D., R. Doyle, and M.H. Floch, Colonic bacterial flora and serum cholesterol: alterations induced by dietary citrus pectin. Am J Gastroenterol, 1985. 80(5): p. 361-4.

222. Goldstein, R.S., J.P. Chism, J.M. Sherrill, and T.E. Hamm, Jr., Influence of dietary pectin on intestinal microfloral metabolism and toxicity of nitrobenzene. Toxicol Appl Pharmacol, 1984. 75(3): p. 547-53.

223. Fukunaga, T., M. Sasaki, Y. Araki, T. Okamoto, T. Yasuoka, T. Tsujikawa, Y. Fujiyama, and T. Bamba, Effects of the soluble fibre pectin on intestinal cell proliferation, fecal short chain fatty acid production and microbial population. Digestion, 2003. 67(1-2): p. 42-9.

224. Manderson, K., M. Pinart, K.M. Tuohy, W.E. Grace, A.T. Hotchkiss, W. Widmer, M.P. Yadhav, G.R. Gibson, and R.A. Rastall, In vitro determination of prebiotic properties of oligosaccharides derived from an orange juice manufacturing by-product stream. Appl Environ Microbiol, 2005. 71(12): p. 83839.

225. Dongowski, G., G. Jacobasch, and D. Schmiedl, Structural stability and prebiotic properties of resistant starch type 3 increase bile acid turnover and lower secondary bile acid formation. J Agric Food Chem, 2005. 53(23): p. 9257-67.

226. Le Blay, G., C. Michel, H.M. Blottiere, and C. Cherbut, Enhancement of butyrate production in the rat caecocolonic tract by long-term ingestion of resistant potato starch. Br J Nutr, 1999. 82(5): p. 419-26. 
227. Lehmann, U., G. Jacobasch, and D. Schmiedl, Characterization of resistant starch type III from banana (Musa acuminata). J Agric Food Chem, 2002. 50(18): p. 5236-40.

228. Kleessen, B., G. Stoof, J. Proll, D. Schmiedl, J. Noack, and M. Blaut, Feeding resistant starch affects fecal and cecal microflora and short-chain fatty acids in rats. J Anim Sci, 1997. 75(9): p. 2453-62.

229. Wang, X., I.L. Brown, D. Khaled, M.C. Mahoney, A.J. Evans, and P.L. Conway, Manipulation of colonic bacteria and volatile fatty acid production by dietary high amylose maize (amylomaize) starch granules. J Appl Microbiol, 2002. 93(3): p. 390-7.

230. Silvi, S., C.J. Rumney, A. Cresci, and I.R. Rowland, Resistant starch modifies gut microflora and microbial metabolism in human flora-associated rats inoculated with faeces from Italian and UK donors. J Appl Microbiol, 1999. 86(3): p. 521-30.

231. Robinson, R.R., J. Feirtag, and J.L. Slavin, Effects of dietary arabinogalactan on gastrointestinal and blood parameters in healthy human subjects. J Am Coll Nutr, 2001. 20(4): p. 279-85.

232. Weaver, G.A., C. Tangel, J.A. Krause, H.D. Alpern, P.L. Jenkins, M.M. Parfitt, and J.J. Stragand, Dietary guar gum alters colonic microbial fermentation in azoxymethane-treated rats. J Nutr, 1996. 126(8): p. 1979-91.

233. Noack, J., B. Kleessen, J. Proll, G. Dongowski, and M. Blaut, Dietary guar gum and pectin stimulate intestinal microbial polyamine synthesis in rats. J Nutr, 1998. 128(8): p. 1385-91.

234. Santosa, S., E. Farnworth, and P.J. Jones, Probiotics and their potential health claims. Nutr Rev, 2006. 64(6): p. 265-74.

235. Janeway, C.A., Jr., Approaching the asymptote? Evolution and revolution in immunology. Cold Spring Harb Symp Quant Biol, 1989. 54(Pt 1): p. 1-13.

236. Takeda, K., T. Kaisho, and S. Akira, Toll-like receptors. Annu Rev Immunol, 2003. 21: p. 335-76.

237. Zarember, K.A. and P.J. Godowski, Tissue expression of human Toll-like receptors and differential regulation of Toll-like receptor mRNAs in leukocytes in response to microbes, their products, and cytokines. J Immunol, 2002. 168(2): p. 554-61.

238. Vinderola, G., C. Matar, and G. Perdigon, Role of intestinal epithelial cells in immune effects mediated by gram-positive probiotic bacteria: involvement of toll-like receptors. Clin Diagn Lab Immunol, 2005. 12(9): p. 1075-84.

239. Takahashi, N., H. Kitazawa, N. Iwabuchi, J.Z. Xiao, K. Miyaji, K. Iwatsuki, and T. Saito, Oral Administration of an Immunostimulatory DNA Sequence from Bifidobacterium longum Improves Th1/Th2 Balance in a Murine Model. Biosci Biotechnol Biochem, 2006. 70(8): p. 2013-7.

240. Rachmilewitz, D., K. Katakura, F. Karmeli, T. Hayashi, C. Reinus, B. Rudensky, S. Akira, K. Takeda, J. Lee, K. Takabayashi, and E. Raz, Toll-like receptor 9 signaling mediates the anti-inflammatory effects of probiotics in murine experimental colitis. Gastroenterology, 2004. 126(2): p. 520-8.

241. Hessle, C., L.A. Hanson, and A.E. Wold, Lactobacilli from human gastrointestinal mucosa are strong stimulators of IL-12 production. Clin Exp Immunol, 1999. 116(2): p. 276-82.

242. Miettinen, M., S. Matikainen, J. Vuopio-Varkila, J. Pirhonen, K. Varkila, M. Kurimoto, and I. Julkunen, Lactobacilli and streptococci induce interleukin-12 (IL-12), IL-18, and gamma interferon production in human peripheral blood mononuclear cells. Infect Immun, 1998. 66(12): p. 6058-62.

243. Mohamadzadeh, M., S. Olson, W.V. Kalina, G. Ruthel, G.L. Demmin, K.L. Warfield, S. Bavari, and T.R. Klaenhammer, Lactobacilli activate human dendritic cells that skew $\mathrm{T}$ cells toward $\mathrm{T}$ helper 1 polarization. Proc Natl Acad Sci U S A, 2005. 102(8): p. 2880-5.

244. Shida, K., J. Kiyoshima-Shibata, M. Nagaoka, K. Watanabe, and M. Nanno, Induction of interleukin12 by lactobacillus strains having a rigid cell wall resistant to intracellular digestion. J Dairy Sci, 2006. 89(9): p. 3306-17.

245. Pochard, P., P. Gosset, C. Grangette, C. Andre, A.B. Tonnel, J. Pestel, and A. Mercenier, Lactic acid bacteria inhibit $\mathrm{TH}_{2}$ cytokine production by mononuclear cells from allergic patients. J Allergy Clin Immunol, 2002. 110(4): p. 617-23. 
246. Cross, M.L., R.R. Mortensen, J. Kudsk, and H.S. Gill, Dietary intake of Lactobacillus rhamnosus $\mathrm{HNOO}_{1}$ enhances production of both $\mathrm{Th}_{1}$ and Th2 cytokines in antigen-primed mice. Med Microbiol Immunol (Berl), 2002. 191(1): p. 49-53.

247. O'Mahony, L., L. O'Callaghan, J. McCarthy, D. Shilling, P. Scully, S. Sibartie, E. Kavanagh, W.O. Kirwan, H.P. Redmond, J.K. Collins, and F. Shanahan, Differential cytokine response from dendritic cells to commensal and pathogenic bacteria in different lymphoid compartments in humans. Am J Physiol Gastrointest Liver Physiol, 2006. 290(4): p. G839-45.

248. Niers, L.E., H.M. Timmerman, G.T. Rijkers, G.M. van Bleek, N.O. van Uden, E.F. Knol, M.L. Kapsenberg, J.L. Kimpen, and M.O. Hoekstra, Identification of strong interleukin-10 inducing lactic acid bacteria which down-regulate T helper type 2 cytokines. Clin Exp Allergy, 2005. 35(11): p. 1481-9.

249. Smits, H.H., A. Engering, D. van der Kleij, E.C. de Jong, K. Schipper, T.M. van Capel, B.A. Zaat, M. Yazdanbakhsh, E.A. Wierenga, Y. van Kooyk, and M.L. Kapsenberg, Selective probiotic bacteria induce IL-10-producing regulatory $\mathrm{T}$ cells in vitro by modulating dendritic cell function through dendritic cell-specific intercellular adhesion molecule 3-grabbing nonintegrin. J Allergy Clin Immunol, 2005. 115(6): p. 1260-7.

250. Daniel, C., S. Poiret, D. Goudercourt, V. Dennin, G. Leyer, and B. Pot, Selecting lactic acid bacteria for their safety and functionality by use of a mouse colitis model. Appl Environ Microbiol, 2006. 72(9): p. 5799-805.

251. Haller, D., Intestinal epithelial cell signalling and host-derived negative regulators under chronic inflammation: to be or not to be activated determines the balance towards commensal bacteria. Neurogastroenterol Motil, 2006. 18(3): p. 184-99.

252. Stadnyk, A.W., Intestinal epithelial cells as a source of inflammatory cytokines and chemokines. Can J Gastroenterol, 2002. 16(4): p. 241-6.

253. Kagnoff, M.F. and L. Eckmann, Epithelial cells as sensors for microbial infection. J Clin Invest, 1997. 100(1): p. 6-10.

254. Tien, M.T., S.E. Girardin, B. Regnault, L. Le Bourhis, M.A. Dillies, J.Y. Coppee, R. Bourdet-Sicard, P.J. Sansonetti, and T. Pedron, Anti-inflammatory effect of Lactobacillus casei on Shigella-infected human intestinal epithelial cells. J Immunol, 2006. 176(2): p. 1228-37.

255. Riedel, C.U., F. Foata, D.R. Goldstein, S. Blum, and B.J. Eikmanns, Interaction of bifidobacteria with Caco-2 cells-adhesion and impact on expression profiles. Int J Food Microbiol, 2006. 110(1): p. 62-8.

256. Frick, J.S., K. Schenk, M. Quitadamo, F. Kahl, M. Koberle, E. Bohn, M. Aepfelbacher, and I.B. Autenrieth, Lactobacillus fermentum attenuates the proinflammatory effect of Yersinia enterocolitica on human epithelial cells. Inflamm Bowel Dis, 2007. 13(1): p. 83-90.

257. Soumelis, V., P.A. Reche, H. Kanzler, W. Yuan, G. Edward, B. Homey, M. Gilliet, S. Ho, S. Antonenko, A. Lauerma, K. Smith, D. Gorman, S. Zurawski, J. Abrams, S. Menon, T. McClanahan, R. de WaalMalefyt Rd, F. Bazan, R.A. Kastelein, and Y.J. Liu, Human epithelial cells trigger dendritic cell mediated allergic inflammation by producing TSLP. Nat Immunol, 2002. 3(7): p. 673-80.

258. Rimoldi, M., M. Chieppa, V. Salucci, F. Avogadri, A. Sonzogni, G.M. Sampietro, A. Nespoli, G. Viale, P. Allavena, and $\mathrm{M}$. Rescigno, Intestinal immune homeostasis is regulated by the crosstalk between epithelial cells and dendritic cells. Nat Immunol, 2005. 6(5): p. 507-14.

259. Rescigno, M., G. Rotta, B. Valzasina, and P. Ricciardi-Castagnoli, Dendritic cells shuttle microbes across gut epithelial monolayers. Immunobiology, 2001. 204(5): p. 572-81.

26o. Rescigno, M., M. Urbano, B. Valzasina, M. Francolini, G. Rotta, R. Bonasio, F. Granucci, J.P. Kraehenbuhl, and P. Ricciardi-Castagnoli, Dendritic cells express tight junction proteins and penetrate gut epithelial monolayers to sample bacteria. Nat Immunol, 2001. 2(4): p. 361-7.

261. Niess, J.H. and H.C. Reinecker, Lamina propria dendritic cells in the physiology and pathology of the gastrointestinal tract. Curr Opin Gastroenterol, 2005. 21(6): p. 687-91. 
262. Niess, J.H., S. Brand, X. Gu, L. Landsman, S. Jung, B.A. McCormick, J.M. Vyas, M. Boes, H.L. Ploegh, J.G. Fox, D.R. Littman, and H.C. Reinecker, $\mathrm{CX}_{3} \mathrm{CR}_{1}$-mediated dendritic cell access to the intestinal lumen and bacterial clearance. Science, 2005. 307(5707): p. 254-8.

263. Macpherson, A.J. and T. Uhr, Compartmentalization of the mucosal immune responses to commensal intestinal bacteria. Ann N Y Acad Sci, 2004. 1029: p. 36-43.

264. Macpherson, A.J. and T. Uhr, Induction of protective IgA by intestinal dendritic cells carrying commensal bacteria. Science, 2004. 303(5664): p. 1662-5.

265. Mazmanian, S.K., C.H. Liu, A.O. Tzianabos, and D.L. Kasper, An immunomodulatory molecule of symbiotic bacteria directs maturation of the host immune system. Cell, 2005. 122(1): p. 107-18.

266. Tyrer, P., A.R. Foxwell, A.W. Cripps, M.A. Apicella, and J.M. Kyd, Microbial pattern recognition receptors mediate $M$-cell uptake of a gram-negative bacterium. Infect Immun, 2006. 74(1): p. 625-31.

267. Jang, M.H., M.N. Kweon, K. Iwatani, M. Yamamoto, K. Terahara, C. Sasakawa, T. Suzuki, T. Nochi, Y. Yokota, P.D. Rennert, T. Hiroi, H. Tamagawa, H. lijima, J. Kunisawa, Y. Yuki, and H. Kiyono, Intestinal villous $M$ cells: an antigen entry site in the mucosal epithelium. Proc Natl Acad Sci U S A, 2004. 101(16): p. 6110-5.

268. Alexander, J.G., Lactobacillus casei tablets in the treatment of intestinal infection. J R Coll Gen Pract, 1971. 21(111): p. 623-4.

269. Perdigon, G., S. Alvarez, M.E. Nader de Macias, M.E. Roux, and A. Pesce de Ruiz Holgado, The oral administration of lactic acid bacteria increase the mucosal intestinal immunity in response to enteropathogens. J Food Prot, 1990. 53(5): p. 404-410.

270. Olivares, M., M.P. Diaz-Ropero, R. Martin, J.M. Rodriguez, and J. Xaus, Antimicrobial potential of four Lactobacillus strains isolated from breast milk. J Appl Microbiol, 2006. 101(1): p. 72-9.

de Waard, R., J. Garssen, J.G. Vos, and E. Claassen, Modulation of delayed-type hypersensitivity and acquired cellular resistance by orally administered viable indigenous lactobacilli in Listeria monocytogenes infected Wistar rats. Lett Appl Microbiol, 2002. 35(3): p. 256-6o.

272. de Waard, R., E. Claassen, G.C. Bokken, B. Buiting, J. Garssen, and J.G. Vos, Enhanced immunological memory responses to Listeria monocytogenes in rodents, as measured by delayed-type hypersensitivity (DTH), adoptive transfer of DTH, and protective immunity, following Lactobacillus casei Shirota ingestion. Clin Diagn Lab Immunol, 2003. 10(1): p. 59-65.

273. Guandalini, S., Probiotics for children: use in diarrhea. J Clin Gastroenterol, 2006. 40(3): p. 244-8.

274. Nomoto, K., Prevention of infections by probiotics. J Biosci Bioeng, 2005. 100(6): p. 583-92.

275. Gill, H.S., Probiotics to enhance anti-infective defences in the gastrointestinal tract. Best Pract Res Clin Gastroenterol, 2003. 17(5): p. 755-73.

276. Katz, J.A., Probiotics for the prevention of antibiotic-associated diarrhea and Clostridium difficile diarrhea. J Clin Gastroenterol, 2006. 40(3): p. 249-55.

277. Van Niel, C.W., C. Feudtner, M.M. Garrison, and D.A. Christakis, Lactobacillus therapy for acute infectious diarrhea in children: a meta-analysis. Pediatrics, 2002. 109(4): p. 678-84.

278. Camilleri, M., Probiotics and irritable bowel syndrome: rationale, putative mechanisms, and evidence of clinical efficacy. J Clin Gastroenterol, 2006. 40(3): p. 264-9.

279. Gionchetti, P., F. Rizzello, K.M. Lammers, C. Morselli, L. Sollazzi, S. Davies, R. Tambasco, C. Calabrese, and M. Campieri, Antibiotics and probiotics in treatment of inflammatory bowel disease. World J Gastroenterol, 2006. 12(21): p. 3306-13.

280. Rioux, K.P. and R.N. Fedorak, Probiotics in the treatment of inflammatory bowel disease. J Clin Gastroenterol, 2006. 40(3): p. 260-3.

281. Isolauri, E., T. Arvola, Y. Sutas, E. Moilanen, and S. Salminen, Probiotics in the management of atopic eczema. Clin Exp Allergy, 2000. 30(11): p. 1604-10. 
282. Rosenfeldt, V., E. Benfeldt, S.D. Nielsen, K.F. Michaelsen, D.L. Jeppesen, N.H. Valerius, and A. Paerregaard, Effect of probiotic Lactobacillus strains in children with atopic dermatitis. J Allergy Clin Immunol, 2003. 111(2): p. 389-95.

283. Viljanen, M., M. Kuitunen, T. Haahtela, K. Juntunen-Backman, R. Korpela, and E. Savilahti, Probiotic effects on faecal inflammatory markers and on faecal IgA in food allergic atopic eczema/dermatitis syndrome infants. Pediatr Allergy Immunol, 2005. 16(1): p. 65-71.

284. Viljanen, M., E. Savilahti, T. Haahtela, K. Juntunen-Backman, R. Korpela, T. Poussa, T. Tuure, and M. Kuitunen, Probiotics in the treatment of atopic eczema/dermatitis syndrome in infants: a doubleblind placebo-controlled trial. Allergy, 2005. 60(4): p. 494-500.

285. Weston, S., A. Halbert, P. Richmond, and S.L. Prescott, Effects of probiotics on atopic dermatitis: a randomised controlled trial. Arch Dis Child, 2005. 90(9): p. 892-7.

286. Kalliomaki, M., S. Salminen, H. Arvilommi, P. Kero, P. Koskinen, and E. Isolauri, Probiotics in primary prevention of atopic disease: a randomised placebo-controlled trial. Lancet, 2001. 357(9262): p. 10769 .

287. Brouwer, M.L., S.A. Wolt-Plompen, A.E. Dubois, S. van der Heide, D.F. Jansen, M.A. Hoijer, H.F. Kauffman, and E.J. Duiverman, No effects of probiotics on atopic dermatitis in infancy: a randomized placebo-controlled trial. Clin Exp Allergy, 2006. 36(7): p. 899-906.

288. Ciprandi, G., M.A. Tosca, M. Milanese, G. Caligo, and V. Ricca, Cytokines evaluation in nasal lavage of allergic children after Bacillus clausii administration: a pilot study. Pediatr Allergy Immunol, 2004. 15(2): p. 148-51.

289. Ciprandi, G., A. Vizzaccaro, I. Cirillo, and M.A. Tosca, Bacillus clausii effects in children with allergic rhinitis. Allergy, 2005. 60(5): p. 702-3.

290. Ciprandi, G., A. Vizzaccaro, I. Cirillo, and M.A. Tosca, Bacillus clausii exerts immuno-modulatory activity in allergic subjects: a pilot study. Allerg Immunol (Paris), 2005. 37(4): p. 129-34.

291. Pohjavuori, E., M. Viljanen, R. Korpela, M. Kuitunen, M. Tiittanen, O. Vaarala, and E. Savilahti, Lactobacillus GG effect in increasing IFN-gamma production in infants with cow's milk allergy. J Allergy Clin Immunol, 2004. 114(1): p. 131-6.

292. Rautava, S., H. Arvilommi, and E. Isolauri, Specific probiotics in enhancing maturation of IgA responses in formula-fed infants. Pediatr Res, 2006. 60(2): p. 221-4.

293. de Vrese, M., P. Winkler, P. Rautenberg, T. Harder, C. Noah, C. Lave, S. Ott, J. Hampe, S. Schreiber, K. Heller, and J. Schrezenmeir, Effect of Lactobacillus gasseri PA 16/8, Bifidobacterium longum SP $07 / 3$, B. bifidum MF 20/5 on common cold episodes: a double blind, randomized, controlled trial. Clin Nutr, 2005. 24(4): p. 481-91.

294. Chiang, B.L., Y.H. Sheih, L.H. Wang, C.K. Liao, and H.S. Gill, Enhancing immunity by dietary consumption of a probiotic lactic acid bacterium (Bifidobacterium lactis HNo19): optimization and definition of cellular immune responses. Eur J Clin Nutr, 2000. 54(11): p. 849-55.

295. Breves, G. and K. Stueck, Short-chain fatty acids in the hindgut, in Physiological and clinical aspects of short-chain fatty acids, J.H. Cummings, J.L. Rombeau, and T. Sakata, Editors. 1995, Cambridge University Press: Cambridge. p. 73-86.

296. Cummings, J.H., Short chain fatty acids in the human colon. Gut, 1981. 22(9): p. 763-79.

297. Rémésy, C., C. Demigné, and C. Morand, Metabolism of short-chain fatty acids in the liver, in Physiological and clinical aspects of short-chain fatty acids, J.H. Cummings, J.L. Rombeau, and T. Sakata, Editors. 1995, Cambridge University Press: Cambridge. p. 171-190.

298. Cummings, J.H., E.W. Pomare, W.J. Branch, C.P. Naylor, and G.T. Macfarlane, Short chain fatty acids in human large intestine, portal, hepatic and venous blood. Gut, 1987. 28(10): p. 1221-7.

299. Basson, M.D., Y.W. Liu, A.M. Hanly, N.J. Emenaker, S.G. Shenoy, and B.E. Gould Rothberg, Identification and comparative analysis of human colonocyte short-chain fatty acid response genes. J Gastrointest Surg, 2000. 4(5): p. 501-12. 
300. Cavaglieri, C.R., A. Nishiyama, L.C. Fernandes, R. Curi, E.A. Miles, and P.C. Calder, Differential effects of short-chain fatty acids on proliferation and production of pro- and anti-inflammatory cytokines by cultured lymphocytes. Life Sci, 2003. 73(13): p. 1683-90.

301. Eftimiadi, C., P. Stashenko, M. Tonetti, P.E. Mangiante, R. Massara, S. Zupo, and M. Ferrarini, Divergent effect of the anaerobic bacteria by-product butyric acid on the immune response: suppression of T-lymphocyte proliferation and stimulation of interleukin-1 beta production. Oral Microbiol Immunol, 1991. 6(1): p. 17-23.

302. Bohmig, G.A., P.M. Krieger, M.D. Saemann, C. Wenhardt, E. Pohanka, and G.J. Zlabinger, n-butyrate downregulates the stimulatory function of peripheral blood-derived antigen-presenting cells: a potential mechanism for modulating T-cell responses by short-chain fatty acids. Immunology, 1997. 92(2): p. 234-43.

303. Millard, A.L., P.M. Mertes, D. Ittelet, F. Villard, P. Jeannesson, and J. Bernard, Butyrate affects differentiation, maturation and function of human monocyte-derived dendritic cells and macrophages. Clin Exp Immunol, 2002. 130(2): p. 245-55.

304. Brisseau, G.F. and O.D. Rotstein, The effects of short-chain fatty acids on phagocytic cell function, in Physiological and clinical aspects of short-chain fatty acids, J.H. Cummings, J.L. Rombeau, and T. Sakata, Editors. 1995, Cambridge University Press: Cambridge. p. 361-372.

305. Nakao, S., A. Fujii, and R. Niederman, Alteration of cytoplasmic Ca2+ in resting and stimulated human neutrophils by short-chain carboxylic acids at neutral pH. Infect Immun, 1992. 60(12): p. 530711.

306. Naccache, P.H., N. Faucher, A.C. Caon, and S.R. McColl, Propionic acid-induced calcium mobilization in human neutrophils. J Cell Physiol, 1988. 136(1): p. 118-24.

307. Niederman, R., J. Zhang, and S. Kashket, Short-chain carboxylic-acid-stimulated, PMN-mediated gingival inflammation. Crit Rev Oral Biol Med, 1997. 8(3): p. 269-9o.

308. Brown, A.J., S.M. Goldsworthy, A.A. Barnes, M.M. Eilert, L. Tcheang, D. Daniels, A.I. Muir, M.J. Wigglesworth, I. Kinghorn, N.J. Fraser, N.B. Pike, J.C. Strum, K.M. Steplewski, P.R. Murdock, J.C. Holder, F.H. Marshall, P.G. Szekeres, S. Wilson, D.M. Ignar, S.M. Foord, A. Wise, and S.J. Dowell, The Orphan $G$ protein-coupled receptors GPR41 and GPR43 are activated by propionate and other short chain carboxylic acids. J Biol Chem, 2003. 278(13): p. 11312-9.

309. Le Poul, E., C. Loison, S. Struyf, J.Y. Springael, V. Lannoy, M.E. Decobecq, S. Brezillon, V. Dupriez, G. Vassart, J. Van Damme, M. Parmentier, and M. Detheux, Functional characterization of human receptors for short chain fatty acids and their role in polymorphonuclear cell activation. J Biol Chem, 2003. 278(28): p. 25481-9.

310. Nilsson, N.E., K. Kotarsky, C. Owman, and B. Olde, Identification of a free fatty acid receptor, FFA2R, expressed on leukocytes and activated by short-chain fatty acids. Biochem Biophys Res Commun, 2003. 303(4): p. 1047-52.

311. Lee, S.K., T. II Kim, Y.K. Kim, C.H. Choi, K.M. Yang, B. Chae, and W.H. Kim, Cellular differentiationinduced attenuation of LPS response in HT-29 cells is related to the down-regulation of TLR4 expression. Biochem Biophys Res Commun, 2005. 337(2): p. 457-63.

312. Sanderson, I.R., Short chain fatty acid regulation of signaling genes expressed by the intestinal epithelium. J Nutr, 2004. 134(9): p. 2450S-2454S.

313. Inan, M.S., R.J. Rasoulpour, L. Yin, A.K. Hubbard, D.W. Rosenberg, and C. Giardina, The luminal short-chain fatty acid butyrate modulates NF-kappaB activity in a human colonic epithelial cell line. Gastroenterology, 2000. 118(4): p. 724-34.

314. Yin, L., G. Laevsky, and C. Giardina, Butyrate suppression of colonocyte NF-kappa B activation and cellular proteasome activity. J Biol Chem, 2001. 276(48): p. 44641-6.

315. Schauber, J., C. Svanholm, S. Termen, K. Iffland, T. Menzel, W. Scheppach, R. Melcher, B. Agerberth, H. Luhrs, and G.H. Gudmundsson, Expression of the cathelicidin LL-37 is modulated by short chain fatty acids in colonocytes: relevance of signalling pathways. Gut, 2003. 52(5): p. 735-41. 
316. Schauber, J., R.A. Dorschner, K. Yamasaki, B. Brouha, and R.L. Gallo, Control of the innate epithelial antimicrobial response is cell-type specific and dependent on relevant microenvironmental stimuli. Immunology, 2006. 118(4): p. 509-19.

317. Willemsen, L.E., M.A. Koetsier, S.J. van Deventer, and E.A. van Tol, Short chain fatty acids stimulate epithelial mucin 2 expression through differential effects on prostaglandin $E(1)$ and $E(2)$ production by intestinal myofibroblasts. Gut, 2003. 52(10): p. 1442-7.

318. Ishizuka, S., S. Tanaka, H. Xu, and H. Hara, Fermentable dietary fiber potentiates the localization of immune cells in the rat large intestinal crypts. Exp Biol Med (Maywood), 2004. 229(9): p. 876-84.

319. Den Hond, E., M. Hiele, P. Evenepoel, M. Peeters, Y. Ghoos, and P. Rutgeerts, In vivo butyrate metabolism and colonic permeability in extensive ulcerative colitis. Gastroenterology, 1998. 115(3): p. 584-90.

320. Scheppach, W., H. Sommer, T. Kirchner, G.M. Paganelli, P. Bartram, S. Christl, F. Richter, G. Dusel, and $\mathrm{H}$. Kasper, Effect of butyrate enemas on the colonic mucosa in distal ulcerative colitis. Gastroenterology, 1992. 103(1): p. 51-6.

321. Harig, J.M., K.H. Soergel, R.A. Komorowski, and C.M. Wood, Treatment of diversion colitis with short-chain-fatty acid irrigation. N Engl J Med, 1989. 320(1): p. 23-8.

322. Breuer, R.I., S.K. Buto, M.L. Christ, J. Bean, P. Vernia, P. Paoluzi, M.C. Di Paolo, and R. Caprilli, Rectal irrigation with short-chain fatty acids for distal ulcerative colitis. Preliminary report. Dig Dis Sci, 1991. 36(2): p. 185-7.

323. Pulverer, G., J. Beuth, W. Roszkowski, H. Burrichter, K. Roszkowski, A. Yassin, H.L. Ko, and J. Jeljaszewicz, Bacteria of human physiological microflora liberate immunomodulating peptides. Zentralbl Bakteriol, 1990. 272(4): p. 467-76.

324. Pulverer, G., H.L. Ko, W. Roszkowski, J. Beuth, A. Yassin, and J. Jeljaszewicz, Digestive tract microflora liberates low molecular weight peptides with immunotriggering activity. Zentralbl Bakteriol, 1990. 272(3): p. 318-27.

325. Pulverer, G., W. Roszkowski, H.J. Beuth, H.L. Ko, and P. Quie, Granulocyte activating factor released from Propionibacterium acnes. A possible mediator of inflammation in acne vulgaris. Zentralbl Bakteriol Mikrobiol Hyg [A], 1988. 270(1-2): p. 246-51.

326. Lee, Y.C. and R.T. Lee, Carbohydrate-protein interactions: basis of glycobiology. Acc Chem Res, 1995. 28(8): p. 321-27.

327. Kilpatrick, D.C., Animal lectins: a historical introduction and overview. Biochim Biophys Acta, 2002. 1572(2-3): p. 187-97.

328. Cooper, D.N. and S.H. Barondes, God must love galectins; he made so many of them. Glycobiology, 1999. 9(10): p. 979-84.

329. Barondes, S.H., D.N. Cooper, M.A. Gitt, and H. Leffler, Galectins. Structure and function of a large family of animal lectins. J Biol Chem, 1994. 269(33): p. 20807-10.

330. Shao, B.M., H. Dai, W. XU, Z.B. Lin, and X.M. Gao, Immune receptors for polysaccharides from Ganoderma lucidum. Biochem Biophys Res Commun, 2004. 323(1): p. 133-41.

331. Xu, Q., T. Yajima, W. Li, K. Saito, Y. Ohshima, and Y. Yoshikai, Levan (beta-2, 6-fructan), a major fraction of fermented soybean mucilage, displays immunostimulating properties via Toll-like receptor 4 signalling: induction of interleukin-12 production and suppression of T-helper type 2 response and immunoglobulin E production. Clin Exp Allergy, 2006. 36(1): p. 94-101.

332. Figdor, C.G., Y. van Kooyk, and G.J. Adema, C-type lectin receptors on dendritic cells and Langerhans cells. Nat Rev Immunol, 2002. 2(2): p. 77-84.

333. Gordon, S., Pattern recognition receptors: doubling up for the innate immune response. Cell, 2002. 111(7): p. 927-30. 
334. Geijtenbeek, T.B., R. Torensma, S.J. van Vliet, G.C. van Duijnhoven, G.J. Adema, Y. van Kooyk, and C.G. Figdor, Identification of DC-SIGN, a novel dendritic cell-specific ICAM-3 receptor that supports primary immune responses. Cell, 2000. 100(5): p. 575-85.

335. Engering, A., T.B. Geijtenbeek, S.J. van Vliet, M. Wijers, E. van Liempt, N. Demaurex, A. Lanzavecchia, J. Fransen, C.G. Figdor, V. Piguet, and Y. van Kooyk, The dendritic cell-specific adhesion receptor DC-SIGN internalizes antigen for presentation to T cells. J Immunol, 2002. 168(5): p. 2118-26.

336. Jensen-Jarolim, E., R. Gscheidlinger, G. Oberhuber, C. Neuchrist, T. Lucas, G. Bises, C. Radauer, M. Willheim, O. Scheiner, F.T. Liu, and G. Boltz-Nitulescu, The constitutive expression of galectin-3 is downregulated in the intestinal epithelia of Crohn's disease patients, and tumour necrosis factor alpha decreases the level of galectin-3-specific mRNA in HCT-8 cells. Eur J Gastroenterol Hepatol, 2002. 14(2): p. 145-52.

337. Gebert, A., The role of M cells in the protection of mucosal membranes. Histochem Cell Biol, 1997. 108(6): p. 455-70.

338. Molis, C., B. Flourie, F. Ouarne, M.F. Gailing, S. Lartigue, A. Guibert, F. Bornet, and J.P. Galmiche, Digestion, excretion, and energy value of fructooligosaccharides in healthy humans. Am J Clin Nutr, 1996. 64(3): p. 324-8.

339. Cebo, C., G. Vergoten, and J.P. Zanetta, Lectin activities of cytokines: functions and putative carbohydrate-recognition domains. Biochim Biophys Acta, 2002. 1572(2-3): p. 422-34.

340. Albers, R., J.M. Antoine, R. Bourdet-Sicard, P.C. Calder, M. Gleeson, B. Lesourd, S. Samartin, I.R. Sanderson, J. Van Loo, F.W. Vas Dias, and B. Watzl, Markers to measure immunomodulation in human nutrition intervention studies. Br J Nutr, 2005. 94(3): p. 452-81. 


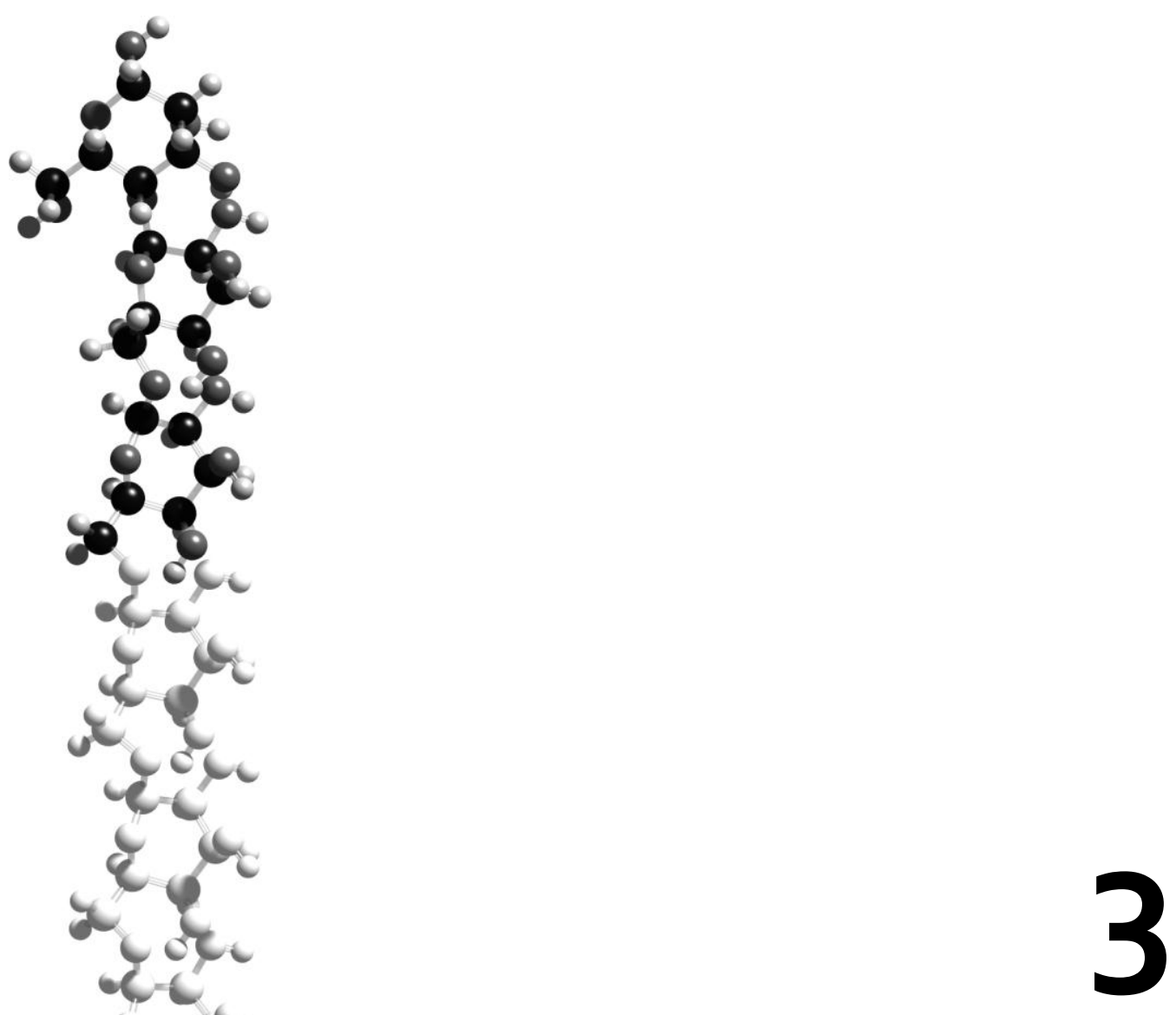

\section{A specific prebiotic oligosaccharide mixture stimulates delayed-type hypersensitivity in a murine influenza vaccination model}

A.P. Vos

M. Haarman

A. Buco

M. Govers

J. Knol

J. Garssen

B. Stahl

G. Boehm

L. M'Rabet

$\underline{\text { Int Immunopharmacol 2006, 6(8):1277-86 }}$ 


\section{Abstract}

Analogous to reported immune-modulatory effects of probiotics, this study was performed to analyze the immune-modulatory properties of prebiotic oligosaccharides that share chemical characteristics with human milk oligosaccharides.

A mixture containing short-chain galactooligosaccharides and long-chain fructooligosaccharides (scGOS/lcFOS; ratio 9:1) was tested at dietary doses between 1 and $10 \%(\mathrm{w} / \mathrm{w}$ of total diet) in an influenza vaccination model, using 10 C56BL/6JolaHsd mice per group. The modulation of vaccine specific delayed-type hypersensitivity (DTH) responses was studied as a marker of T-helper 1 (Th1) immunity, as well as other immune parameters. scGOS/lcFOS enhanced DTH responses dose-dependently (optimum at 5\% w/w of total diet; $41.4 \pm 14.1 \%$ increased compared to controls, $\mathrm{p}<0.05)$. No significant changes were detected on splenocyte proliferation or vaccine-specific antibody concentrations. Simultaneously, scGOS/lcFOS dose-dependently increased the proportion of fecal bifidobacteria and lactobacilli (maximal effect at $10 \% \mathrm{w} / \mathrm{w}$ of total diet; $16.8 \pm 2.4 \%$ and $5.8 \pm 1.3 \%$ increased compared to controls respectively, $\mathrm{p}<0.01$ for both parameters)

In a comparative experiment, scGOS/lcFOS and scFOS/inulin (both at $2 \% \mathrm{w} / \mathrm{w}$ of total diet) induced similar significant effects on the gut microbiota. In contrast to scGOS/lcFOS, scFOS/inulin did not enhance DTH responses, indicating that an increase in the proportions of bifidobacteria and lactobacilli is not sufficient for an immunemodulatory effect in this model.

The use of scGOS/lcFOS in dietary products might provide an opportunity to stimulate the adaptive immune response in a Th1-direction and subsequently inhibit infections and Th2-related immune disorders in humans, for instance allergies. Clinical studies are being performed to confirm this.

\section{Introduction}

Oligosaccharides are a major constituent of human milk and are thought to play an important role in its growth-promoting effect on bifidobacteria and lactobacilli in the infant's gut $(1,2)$. Prebiotic substances have a similar effect on the microbiota of the gut, since they are defined as "non-digestible food ingredients that beneficially affect the host by selectively stimulating the growth and/or activity of one or a limited number of 
bacteria in the colon (3). Examples of well-studied prebiotics are inulin, fructooligosaccharides (FOS) and galactooligosaccharides (GOS). These have been shown to increase the number of bifidobacteria and lactobacilli in the intestinal microbiota of animals and man (4-8).

Living bifidobacteria and lactobacilli applied as probiotic agents have immunemodulatory effects on innate and mucosal immune parameters (9), as well as on systemic immune responses (10). It is assumed that prebiotics have effects on the immune system similar to probiotics, because both enhance the number of 'beneficial' bacteria in the gut. However, the relation between prebiotic agents and immunity has not been documented in detail. Literature reports have focused mainly on the mucosal part of the immune system, and actual prebiotic effects on the intestinal microbiota were not analyzed simultaneously in most studies (11-15).

Therefore, we tested whether a specific prebiotic mixture (scGOS/lcFOS in a ratio 9:1; Immunofortis) modulates systemic immune responses in a murine vaccination model, analogous to published effects of probiotics (10). The use of vaccination response parameters for studying immune modulation in nutritional intervention studies was recently advocated by an ILSI/WHO expert group $(16,17)$. We established an influenza vaccination model in young adult C57BL/6JOlaHsd mice. Because dietary intervention could potentially lead to either down-regulation or up-regulation of the immune responses, conditions had to be selected in such a way that both types of modulation could be detected. Therefore, the vaccine dose was titrated in an initial experiment to induce a sub-maximal immune response. Modulation of cellular immune responses to vaccination was studied by delayed-type hypersensitivity (DTH) responses, as a parameter for cellular T-helper1 (Th1) dependent immunity, and by vaccine-induced splenocyte proliferation as a parameter for T-cell activation. Modulation of the humoral immune response to vaccination was measured by vaccine-specific serum antibody concentrations.

To be able to correlate the immune related parameters with changes in the microbiota, the intestinal microbiota were measured simultaneously with the immune related parameters in each experiment. Prebiotic effects were studied by measuring bacterial composition and biochemical parameters in fecal or colon samples.

scGOS/lcFOS is a mixture of short-chain GOS and long-chain FOS in a 9:1 ratio, that was designed to resemble the neutral fraction of human milk oligosaccharides (HMOS) with regard to its molecular size distribution and high galactose residue content (1820). When applied in an infant milk formula (IMF), this mixture has been shown to functionally mimic the effects of breast-feeding on the fecal flora in term and preterm infants (20-23). The dose range that was tested in this experiment (1 to $10 \% \mathrm{w} / \mathrm{w}$ of the 
scGOS/lcFOS product in total diet) related to oligosaccharide doses in human milk and infant milk formulas (IMF). Human milk contains around $1.2 \mathrm{~g}$ oligosaccharides per $100 \mathrm{~mL}$ (corresponding to approximately $10 \%$ of the dry weight) $(24,25)$. Doses of scGOS/lcFOS in infant studies were 0.4 and $0.8 \mathrm{~g}$ oligosaccharides per $100 \mathrm{~mL}$ IMF (corresponding to 3 and $6 \%$ of the dry weight). To study whether the observed effects were specific to scGOS/lcFOS supplementation, three other commercially available FOS or inulin products were tested in comparison, which have also been described to induce prebiotic effects $(26,27)$.

\section{Materials and methods}

\section{Mice}

Female 6- and 8-week old C57Bl//6JOlaHsd mice were obtained from Harlan (Horst, The Netherlands) and kept under normal conditions with a $12 \mathrm{~h}$ dark and light cycle and free access to food and water. All experiments were approved by an independent animal experiments committee (DEC Consult, Bilthoven, The Netherlands).

\section{Diets and oligosaccharide preparations}

All animals received semi-purified AIN-93G-based diets (Research Diet Services, Wijk bij Duurstede, The Netherlands). All supplemented oligosaccharide products were exchanged for the same amount of total carbohydrates, to keep this parameter equal. In addition, this approach resulted in a comparable overall carbohydrate composition in different diets, to ensure that the gut flora was minimally influenced by differences between control and test diets in parameters such as gut passage time and fluid retention. The oligosaccharides were mixed into the AIN-93G diet and pressed into pellets. Deviations from the AIN-93G specification are specified in Table 1 for all supplemented diets.

scGOS/lcFOS (Immunofortis) is a spray-dried powder of short-chain GOS (Vivinal GOS, Borculo Domo, Zwolle, The Netherlands) with a degree of polymerization (dp) of 3-8, and long-chain FOS (Raftiline HP, Orafti, Wijchen, The Netherlands; average dp $>23$ ) in a 9:1 ratio. It consists of approximately 50\% (w/w of product) scGOS and lcFOS. Non-oligosaccharide carrier materials in scGOS/lcFOS are approximately $19 \%$ (w/w of product) maltodextrin (Glucidex 2, Roquette, France), 16\% (w/w of product) lactose, $14 \%$ (w/w of product) glucose and $1 \%(\mathrm{w} / \mathrm{w}$ of product) galactose. scFOS/inulin (Raftilose Synergy 1,Orafti) consists for approximately $92 \%$ (w/w of product) of a 
Table 1. Composition of the diets

\begin{tabular}{|c|c|c|c|c|c|c|c|}
\hline \multirow[b]{2}{*}{ Ingredients (g/kg) } & \multirow{2}{*}{$\begin{array}{c}\text { Control } \\
\text { diets (AIN- } \\
93 \mathrm{G})\end{array}$} & \multicolumn{4}{|c|}{ scGOS/lcFOS dose-response diets } & \multirow{2}{*}{$\begin{array}{l}\text { Diets containing } \\
\qquad 2 \% \\
\text { oligosaccharide } \\
\text { preparations }\end{array}$} & \multirow{2}{*}{$\begin{array}{c}\text { Diet } \\
\text { containing } \\
\text { carrier } \\
\text { material }\end{array}$} \\
\hline & & $1 \%$ & $2.5 \%$ & $5 \%$ & $10 \%$ & & \\
\hline Cornstarch & 397.5 & 391.6 & 382.9 & 368.2 & 339.0 & 385.8 & 391.6 \\
\hline Dextrinized cornstarch & 132.0 & 130.1 & 127.1 & 122.3 & 112.6 & 128.1 & 130.1 \\
\hline Sucrose & 100.0 & 98.5 & 96.3 & 92.6 & 85.3 & 97.1 & 98.5 \\
\hline Cellulose & 50.0 & $49 \cdot 3$ & 48.2 & 46.3 & 42.6 & 48.5 & $49 \cdot 3$ \\
\hline Added carbohydrates & & $10^{a}$ & $25^{a}$ & $50^{a}$ & $100^{a}$ & $20^{b}$ & $10^{c}$ \\
\hline
\end{tabular}

${ }^{a}$ scGOS/lcFOS; ${ }^{b}$ one of the following ingredients: scGOS/lcFOS, scFOS/inulin, scFOS or inulin; 'a combination of $3.8 \mathrm{~g} / \mathrm{kg}$ maltodextrin, $3.2 \mathrm{~g} / \mathrm{kg}$ lactose, $2.8 \mathrm{~g} / \mathrm{kg}$ glucose and $0.2 \mathrm{~g} / \mathrm{kg}$ galactose. Please refer to the text for details on the added carbohydrates.

combination of inulin and FOS. Short-chain FOS (scFOS; Raftilose P95, Orafti) contains 95\% (w/w of product) scFOS (dp 2-7). Inulin (Raftiline ST, Orafti) contains around 92\% $(w / w$ of product) FOS with a broad range of chain-lengths (average $d p=10$ ). The remainder of the latter three products consists of glucose, fructose and sucrose.

The dosages mentioned in this chapter reflect the percentages of the total products in the diets.

\section{Vaccination protocol and DTH response}

Vaccination experiments were performed using Influvac (Solvay Pharmaceuticals, Weesp, the Netherlands) from season 2002/2003. It is an inactivated influenza virus vaccine based on isolated haemagglutinin (HA) and neuraminidase antigens of three strains of myxovirus influenza, in a dose equivalent to $30 \mu \mathrm{g} / \mathrm{ml} \mathrm{HA}$ per strain (90 $\mu \mathrm{g} / \mathrm{ml} \mathrm{HA}$ in total). An oil-adjuvant was used in all vaccinations (Stimune, previously known as Specol; Cedi-diagnostics, Lelystad, The Netherlands).

The mice received a primary vaccination and a booster vaccination, consisting of a subcutaneous (sc) injection of a 1:1 mix of vaccine and adjuvant in a total volume of 100 $\mu \mathrm{L}$. The booster vaccination was given 21 days after the primary vaccination. The experiments ended 10 days after booster vaccination. Blood samples (taken by retroorbital puncture) were taken before primary and secondary vaccination and at the end of the experiment. Negative control groups that were included in all experiments (indicated with 'sham group') received injections with a 1:1 mix of PBS and adjuvant in a total volume of $100 \mu \mathrm{L}$. Sham groups were never used for statistical comparisons to supplemented groups, but served solely to demonstrate the specificity of vaccine- 
induced responses.

DTH reactions were induced 9 days after booster vaccination, by sc injection of $25 \mu \mathrm{L}$ Influvac into the ear pinnae of both ears. Ear thickness was measured in duplicate before vaccine challenge and 24 hours thereafter, using a digital micrometer (Mitutoyo Digimatic 293561, Veenendaal, The Netherlands). The DTH response was calculated by subtracting the basal ear thickness from the value at 24 hours after challenge.

\section{Vaccine titration experiment}

In the initial experiment, 33 mice were randomly assigned to 3 experimental groups (8 weeks of age; $n=10)$ and a sham group $(n=3)$. Three dilutions of the vaccine were tested, with the objective to find a dose that induces sub-maximal DTH and antibody responses. Vaccine concentrations of $0.9,9$ and $90 \mu \mathrm{g} / \mathrm{ml}$ HA (corresponding to 100x, 10x and $1 \mathrm{x}$ diluted vaccine) were tested in PBS, keeping the injection volume and amount of adjuvant constant. Vaccine concentration rather than the amount of adjuvant was varied because it allowed the use of a constant injection volume.

\section{Dietary intervention experiments}

The first dietary intervention experiment was performed to test the effect of different doses of the scGOS/lcFOS mixture on vaccine-induced immune responses. Fifty-three mice were randomly assigned to 4 experimental groups and 1 control group (6 weeks of age; $n=10)$, as well as a sham group $(n=3)$. Mice in control and sham groups received the AIN-93G control diet without supplements.

Subsequent experiments were performed to compare the observed effects of the scGOS/lcFOS mixture with effects of other oligosaccharide preparations. Supplementation of $2 \%$ (w/w of total diet) scGOS/lcFOS was compared with inulin/FOS, scFOS and inulin, each at $2 \%$ (w/w of total diet). In addition, scGOS/lcFOS supplementation $(2 \% \mathrm{w} / \mathrm{w}$ of total diet) was compared with supplementation of the same amount carrier material that is present in $2 \%(\mathrm{w} / \mathrm{w}$ of total diet) scGOS/lcFOS mixture. Both groups were also compared to the unsupplemented control diet.

In all experiments, the $0.9 \mu \mathrm{g} / \mathrm{ml}$ Influvac dose was used (100x diluted vaccine). All animals received the control diet for a minimum of 5 days before the start of the dietary interventions. Dietary supplementation started 20 days (scGOS/lcFOS dose response experiment) or 14 days (other experiments) before the first vaccination and lasted until the end of the experiment, 31 days after the first vaccination. 


\section{Antigen-specific antibody measurements}

Serum antibody concentrations were measured by ELISA. In short, 96-well plates (Costar EIA/RIA plate, Alphen a/d Rijn, The Netherlands) were coated with 1:100 diluted Influvac in PBS. Blocking reagent was 2\% BSA (Sigma, Zwijndrecht, The Netherlands) in PBS. A dilution series of pooled serum that contained anti-vaccine antibodies was added for standard curve calculation. Anti-IgG-HRP (Santa Cruz Biotechnology, Heerhugowaard, The Netherlands), anti-IgG1-biotin and anti-IgG2abiotin (Becton Dickinson, Heerhugowaard, The Netherlands) antibodies were diluted 1:1000 in dilution buffer. For the biotin-conjugated antibodies, the plates were subsequently incubated with a 1:20,000 dilution of streptavidin-HRP (Biosource, EttenLeur, The Netherlands). Plates were incubated with ready-to-use TMB substrate (Perbio Science, Etten-Leur, The Netherlands) and were measured in a Bio-Rad Ultramark plate reader. Concentrations in test sera were calculated in arbitrary units (AU), relative to the standard curve of the diluted pooled serum. The concentration of the pooled serum was defined as 1,000 AU/ml.

\section{Splenocyte proliferation assay}

Splenocytes were isolated by gently pressing spleen tissue through nylon mesh filters (Falcon cell strainer, Becton Dickinson, Alphen a/d Rijn, the Netherlands). After erythrocyte lysis by osmotic shock, cells were counted and diluted to the appropriate concentrations. Cultures were incubated with Influvac vaccine at a predetermined optimal concentration, and proliferation was measured by tritiated thymidine incorporation. In short, $1^{*} 10^{6}$ cells were distributed in 96-well plates in triplicate in RMPI-1640 medium (Invitrogen, Merelbeke, Belgium) supplemented with $10 \%$ fetal calf serum, $100 \mathrm{U} / \mathrm{ml}$ penicillin and $100 \mu \mathrm{g} / \mathrm{mL}$ streptomycin. Cells were stimulated with control medium or with $0.1 \mu \mathrm{g} / \mathrm{mL}$ Influvac vaccine solution. After four days of culture, $0.4 \mu \mathrm{Ci} /$ well tritiated thymidine was added, and after 24 hours cells were harvested (Filtermate, Perkin Elmer) and counted in a scintillation counter (Wallac Microbeta, Perkin Elmer). Data are presented as the difference in counts per minute (cpm) between control medium and Influvac-stimulated wells.

\section{Feces collection, $\mathrm{pH}$ measurement and analysis of SCFAs and lactate}

In the first experiment (scGOS/lcFOS dose-response study), fecal samples were collected from the cages from the last two days of the study. In the second experiment (comparison of scGOS/lcFOS with other oligosaccharide preparations), samples were collected freshly from the colon during dissection. All samples were stored at $-20{ }^{\circ} \mathrm{C}$. 
After thawing, the samples were pooled per experimental group (using a fixed weight of sample per animal) and 10x diluted in saline (w/v). The $\mathrm{pH}$ was measured in the diluted samples at room temperature.

For short-chain fatty acid (SCFA) analysis, $350 \mu \mathrm{L}$ of fecal suspension was mixed with $200 \mu \mathrm{L} 5 \%(\mathrm{v} / \mathrm{v})$ formic acid, $100 \mu \mathrm{L} 1.25 \mathrm{~g} / \mathrm{L}$ 2-ethylbutyric acid (as internal standard, Sigma-Aldrich, Zwijndrecht, The Netherlands) and $350 \mu \mathrm{L}$ MilliQ water. The samples were centrifuged for $5 \mathrm{~min}$ at 16,000 xg to remove large particles and the supernatants were stored at $-20^{\circ} \mathrm{C}$.

SCFAs were quantitatively determined by a Varian 3800 gas chromatograph (GC) (Varian, Inc., Walnut Creek, U.S.A.) equipped with a flame ionization detector. $0.5 \mu \mathrm{L}$ of the sample was injected at $80{ }^{\circ} \mathrm{C}$ in the column (Stabilwax, $15 \mathrm{~m} \times 0.53 \mathrm{~mm}$, film thickness $1.00 \mu \mathrm{m}$, Restek Co., USA) using helium as carrier gas (3.0 psi). After injection of the sample, the oven was heated to $160{ }^{\circ} \mathrm{C}$ at a speed of $16{ }^{\circ} \mathrm{C} / \mathrm{min}$, followed by heating to $220{ }^{\circ} \mathrm{C}$ at a speed of $20{ }^{\circ} \mathrm{C} / \mathrm{min}$ and finally maintained at a temperature of 220 ${ }^{\circ} \mathrm{C}$ for 1.5 minutes. The temperature of the injector and the detector was $200{ }^{\circ} \mathrm{C}$. Data are expressed in $\mathrm{mmol} / \mathrm{g}$ of fecal weight.

For lactate analysis, the fecal suspension was centrifuged for 5 minutes at 16,000 xg. 100 $\mu \mathrm{L}$ supernatant was heated for 10 minutes at $100{ }^{\circ} \mathrm{C}$ to inactivate all enzymes. Concentrations of L-lactic acid and D-lactic acid were determined enzymatically using a L-lactic acid detection kit and D-lactate-dehydrogenase (Scil Diagnostics GmbH, Viernheim Germany). Data are expressed in $\mathrm{mmol} / \mathrm{g}$ of fecal weight.

\section{Feces preparation and DNA isolation for QPCR analysis of microbiota}

Frozen fecal or colon samples were thawed by adding $1 \mathrm{~mL}$ of MilliQ water and heating at $90{ }^{\circ} \mathrm{C}$. The suspensions were subsequently homogenized and frozen overnight at $-20{ }^{\circ} \mathrm{C}$. The homogenized samples were thawed at room temperature, followed by DNA isolations using the NucliSense Isolation Extraction Kit (BioMerieux, Boxtel, The Netherlands).

\section{Quantitative real-time PCR of the genus Bifidobacterium and Lactobacillus}

For the relative quantification of the genera Bifidobacterium (28) and Lactobacillus (29) in relation to the total bacterial load, a duplex $5^{\prime}$ nuclease quantitative real-time PCR (qPCR) assay was used.

Briefly, different primers and probes for the genus Bifidobacterium or Lactobacillus in combination with primers and probes for the total bacterial load (30) were used in a temperature profile consisting of $2 \mathrm{~min}$ at $50^{\circ} \mathrm{C}, 10 \mathrm{~min}$ at $95^{\circ} \mathrm{C}$, followed by 45 cycles 
of $15 \mathrm{~s}$ at $95^{\circ} \mathrm{C}$ and $60^{\circ} \mathrm{C}$ for $1 \mathrm{~min}$, run on ABI Prism 7700 PCR equipment (Applied Biosystems, Nieuwerkerk a/d IJssel, The Netherlands).

Thereafter, the relative amounts of the genus Bifidobacterium or Lactobacillus in the samples were calculated with respect to the total bacterial load according to Liu et al. (31), and expressed in percentages. All samples were measured in triplicate.

\section{Statistical analysis}

Statistical calculations were performed using SPSS version 12.0.1 software. Antibody concentration data were log-transformed to normalize the distribution, before statistical calculations were performed. Statistical differences between test and control groups were analyzed by ANOVA and post-hoc Dunnett's test if multiple groups were compared to a single (control) group. Bonferroni post-hoc tests were performed after ANOVA if comparisons were made to multiple groups. Dose-response relationships were analyzed by linear and quadratic regression, to analyze linear or curved relationships. Based on residual squares analysis, the quadratic model provided the best fit in all cases. Regression curves with 95\% confidence intervals (CI) are plotted in the graphs. The significance of differences between individual dose-groups and the control group in the dose-response experiment was tested using post-hoc Dunnett's tests. P-values $<0.05$ were considered significant in all experiments. All statistical tests in this study were performed two-sided.

\section{Results}

\section{Dose-dependent vaccine responses}

In the dose range of 0.9 to $90 \mu \mathrm{g} / \mathrm{mL}$, there was a clear dose-response relationship between the vaccine dose and both DTH and antibody responses (Fig. 1). Already at the lowest dose of $0.9 \mu \mathrm{g} / \mathrm{mL}$, a response was detectable in DTH and IgG-class antivaccine antibodies. These responses were significantly lower than those at higher vaccine doses. This was also observed in vaccine-specific IgG1- and IgG2a-subclass antibody concentrations, but differences between groups were not statistically significant, due to higher variation (data not shown). In addition, vaccine-specific splenocyte proliferation was measured ex vivo. Proliferation responses in the $90 \mu \mathrm{g} / \mathrm{mL}$ vaccine group were $1.1^{*} 10^{4} \pm 1.6^{*} 10^{3} \mathrm{cpm}$, compared to $-202 \pm 85 \mathrm{cpm}$ (mean \pm SEM) in the sham group. No significant differences were found between the different vaccination doses. 


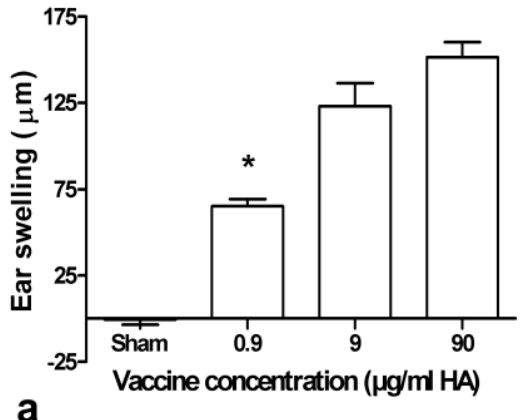

a

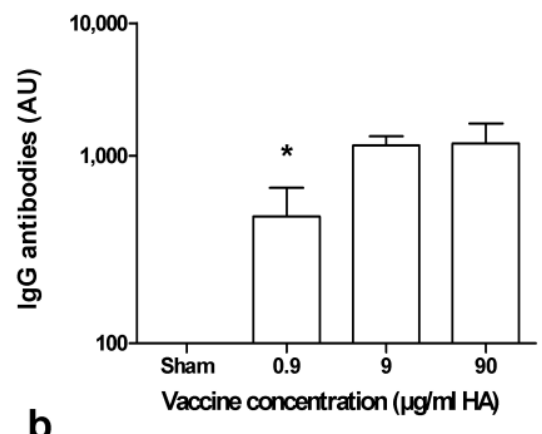

b

Figure 1. DTH responses and serum antibody concentrations after vaccination with 3 ditterent vaccine concentrations. (a) DTH responses measured by ear swelling. (b) Vaccine-specific total IgG concentration in arbitrary units $(\mathrm{AU})$. The concentrations were calculated relative to a pooled serum from vaccinated animals, with a defined concentration of 1,000 AU. * Indicates $p<0.05$ compared to the higher dose group, tested using ANOVA and post hoc Dunnett's test; error bars indicate $S E M ; n=3$ in sham groups, $n=10$ in all other groups.

Low background responses were shown for all parameters in the sham groups, which indicates that the responses were vaccine-specific. This was also the case in subsequent dietary intervention experiments (data not shown). Thus, the $0.9 \mu \mathrm{g} / \mathrm{mL}$ vaccine dose induced sub-maximal and specific DTH and IgG-class antibody responses. Therefore, this dose was used in all subsequent experiments, to enable the detection of increased as well as decreased vaccination responses in dietary intervention studies.

\section{Animal weights and feed intake}

Animal weights and feed intake were monitored weekly during all dietary intervention experiments. No significant differences were measured between experimental groups within the same experiment for both parameters, indicating that all diets were well tolerated. Animal weight at the end of the experiment and average feed intake per week are presented in Table 2.

\section{DTH responses}

A dose-dependent effect on the DTH response was found in the dietary supplementation range between 1 and 10\% (w/w of total diet) scGOS/lcFOS (Fig. 2a). Regression analysis of the experiment suggested an optimum at the $5 \%(\mathrm{w} / \mathrm{w}$ of total diet) scGOS/lcFOS dose. This dose significantly increased the DTH response when 
Table 2. Animal weights and feed intake

\begin{tabular}{|c|c|c|c|c|c|}
\hline \multirow[b]{2}{*}{ Experiment } & \multirow[b]{2}{*}{ Group } & \multicolumn{2}{|c|}{$\begin{array}{c}\text { Animal weight at the end } \\
\text { of the experiment }\end{array}$} & \multicolumn{2}{|c|}{$\begin{array}{l}\text { Weekly feed intake per } \\
\text { animal }\end{array}$} \\
\hline & & Mean (g) & SEM & Mean (g) & SEM \\
\hline \multirow{5}{*}{$\begin{array}{l}\text { scGOS/lcFOS dose } \\
\text { response experiment }\end{array}$} & Control & 19.62 & 0.33 & 17.91 & 0.60 \\
\hline & $1 \%$ & $19 \cdot 44$ & 0.37 & 17.82 & 0.62 \\
\hline & $2.5 \%$ & 19.90 & 0.32 & 17.83 & 0.50 \\
\hline & $5 \%$ & 20.14 & 0.48 & 18.21 & 0.51 \\
\hline & $10 \%$ & 20.27 & 0.46 & 18.38 & $0.5^{8}$ \\
\hline \multirow{5}{*}{$\begin{array}{l}\text { Comparison of different } \\
\text { oligosaccharide } \\
\text { preparations }\end{array}$} & Control & 19.71 & 0.59 & 17.42 & 0.44 \\
\hline & scGOS/lcFOS & 19.68 & 0.27 & 17.10 & 0.45 \\
\hline & scFOS/inulin & $19 \cdot 36$ & 0.43 & 17.08 & 0.39 \\
\hline & scFOS & 19.87 & 0.28 & 17.74 & 0.41 \\
\hline & Inulin & 19.04 & 0.41 & 17.02 & 0.41 \\
\hline \multirow{3}{*}{$\begin{array}{l}\text { Comparison of } \\
\text { scGOS/lcFOS and carrier } \\
\text { material }\end{array}$} & Control & 18.98 & 0.39 & 16.47 & 0.30 \\
\hline & scGOS/lcFOS & 19.23 & 0.41 & 16.30 & 0.33 \\
\hline & Carrier & 18.52 & 0.35 & 16.51 & 0.38 \\
\hline
\end{tabular}

Feed intake was measured weekly per animal cage; the data are shown as the mean intake per animal per week. No significant differences in animal weight or feed intake were observed between experimental groups within an experiment, as analysed by ANOVA.

compared to the control group. Mice that were fed with lower doses of scGOS/lcFOS also showed higher DTH responses than mice that received the control diet, but the differences were not significant. In subsequent experiments with a similar experimental design and identical procedure, doses between $1 \%$ and $2.5 \%(\mathrm{w} / \mathrm{w}$ of total diet) scGOS/lcFOS also showed significantly increased DTH responses compared to control mice (data not shown; see also Fig. 2b).

The immune-modulatory effect of scGOS/lcFOS in the vaccination model was compared with other commercially available oligosaccharides. Three different FOS and inulin preparations were compared with scGOS/lcFOS at a fixed supplementation dose of $2 \%$ (w/w of total diet). Only scGOS/lcFOS supplementation resulted in a significant increase of the DTH response (Fig. $2 b$ ). In addition, $2 \%$ (w/w of total diet) scGOS/lcFOS supplementation was compared to the same amount of carrier material present in the scGOS/lcFOS mixture. Both groups were also compared to the unsupplemented control diet. The carrier material did not induce a significant increase in the DTH response compared to the unsupplemented diet. In contrast, the DTH responses in mice that 
received $2 \%(\mathrm{w} / \mathrm{w}$ of total diet) scGOS/lcFOS were significantly higher than the responses in both other groups (Fig. 2c).

\section{Splenocyte proliferation}

Ex-vivo vaccine-induced proliferation was measured after 5 days of culture. Low background proliferation was measured in sham-vaccinated mice (179 \pm 470 cpm; mean \pm SEM). Clear proliferative responses were measured in the Influvac-vaccinated control mice $\left(5.0^{*} 10^{4} \pm 823 \mathrm{cpm}\right.$; mean \pm SEM). No significant changes in proliferative responses were measured in scGOS/lcFOS supplemented mice compared to controls.

\section{Serum antibody concentrations}

In the scGOS/lcFOS dose-response experiment, vaccine-specific antibody concentrations were measured in serum after primary vaccination and at the end the intervention period. Total IgG concentrations were $116 \pm 12$ and $1176 \pm 506$ AU (mean \pm SEM) in the control groups after primary and secondary vaccination, respectively. Corresponding concentrations were $115 \pm 21$ and $1245 \pm 545$ AU (mean \pm SEM) for the $\mathrm{IgG}_{1}$ subclass and $140 \pm 66$ and $1058 \pm 482 \mathrm{AU}$ (mean \pm SEM) for the IgG2a subclass. High intra-group variation was observed in all experimental groups and no significant differences between groups were found for any antibody subclass (data not shown). IgG2a antibodies are generally associated with Th1 activity, while IgG1 antibody responses are associated with Th2 activity, based on the ability of IFN- $\gamma$ and IL-4 to stimulate and cross-regulate the production of these $\operatorname{IgG}$ subclasses $(32,33)$. Therefore, IgG1/IgG2a ratios were calculated for each individual animal in the current experiment. The ratio was $1.12 \pm 0.64$ (mean \pm SEM) in the control group; no significant differences were found between groups (data not shown). 

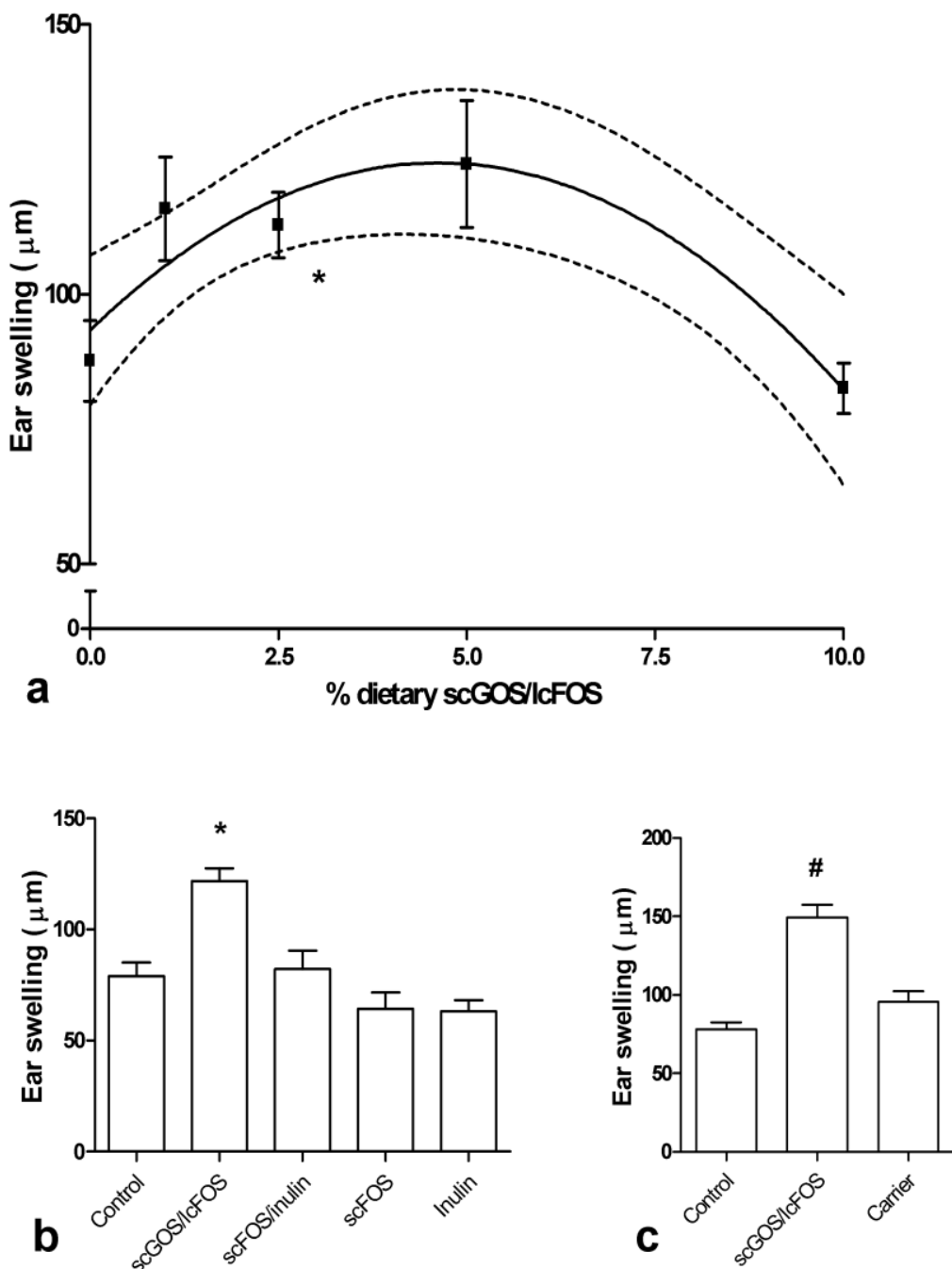

Figure 2. Effect of dietary intervention with oligosaccharide preparations on DTH responses. (a) Dose-reponse of scGOS/lcFOS (\% w/w of total diet). Quadratic regression was performed to analyse dose-response relationship and the optimal fitting curve is plotted in the graph together with the $95 \% \mathrm{Cl}$ s. (b) Comparison of scGOS/lcFOS with 3 other oligosaccharide preparations, at $2 \%$ ( $w / w$ of total diet) in the diet. (c) Comparison of $2 \%$ (w/w of total diet) scGOS/lcFOS supplementation with a corresponding amount of non-oligosaccharide carrier materials that is present in $2 \%$ scGOS/lcFOS (consisting mainly of maltodextrin, lactose, glucose). Both diets were compared to unsupplemented AIN-93G control diet. In (a) and (b), * indicates $p<0.05$ compared to the control group; tested with ANOVA and post hoc Dunnett's test. In (c), \# indicates $p<0.05$ compared to all other groups, tested with ANOVA and post hoc Bonferroni's test. In all graphs, error bars indicate SEM and $n=10$ in all groups. 


\section{Microbiota-related parameters}

The proportion of bifidobacteria in pooled fecal samples from the control group in the scGOS/lcFOS dose-response study was high (27.6 $\pm 3.0 \%$; mean \pm SEM) compared with colon samples from control mice in the comparison experiment of scGOS/lcFOS with other oligosaccharides $(3.2 \pm 0.6 \%$; mean $\pm S E M)$. This aside, dietary intervention with increasing doses of scGOS/lcFOS elevated the number of bifidobacteria in the pooled fecal samples in a dose-dependent manner (Fig. 3a). Post-hoc analysis showed that all dose groups contained significantly higher proportions of fecal bifidobacteria than the control group. scGOS/lcFOS also elevated the proportion of lactobacilli in the pooled samples in a dose-dependent manner; the effect was statistically significant in the $10 \%$ group (Fig. 3b).

Sugar-fermenting bacteria, including lactobacilli and bifidobacteria, are known to produce lactate and SCFAs in the gut, which results in a decreased $\mathrm{pH}$ of the gut content. The fecal pH decreased from 8.4 in the control group to 7.3 in the $10 \%$ scGOS/lcFOS group (Fig. 3c). SCFA concentrations (acetic acid, propionic acid and butyric acid) were mainly increased in the $10 \%$ scGOS/lcFOS group (Fig. 3d). Finally, lactate concentrations were increased in the 2.5, 5 and $10 \%$ groups compared to controls (Fig. 3e). Due to a limited amount of sample material, no repeated measurements and therefore no statistics were performed on these biochemical parameters.

scGOS/lcFOS was compared with three other oligosaccharide preparations at a supplementation dose of $2 \%$ (w/w of total diet), regarding the colon microbiota content. Both scGOS/lcFOS and scFOS/inulin significantly increased the relative numbers of bifidobacteria (Fig. 4a) and lactobacilli (Fig. 4b), compared to the control diet. scFOS and inulin showed no significant effects on both groups of bacteria at this dose. 

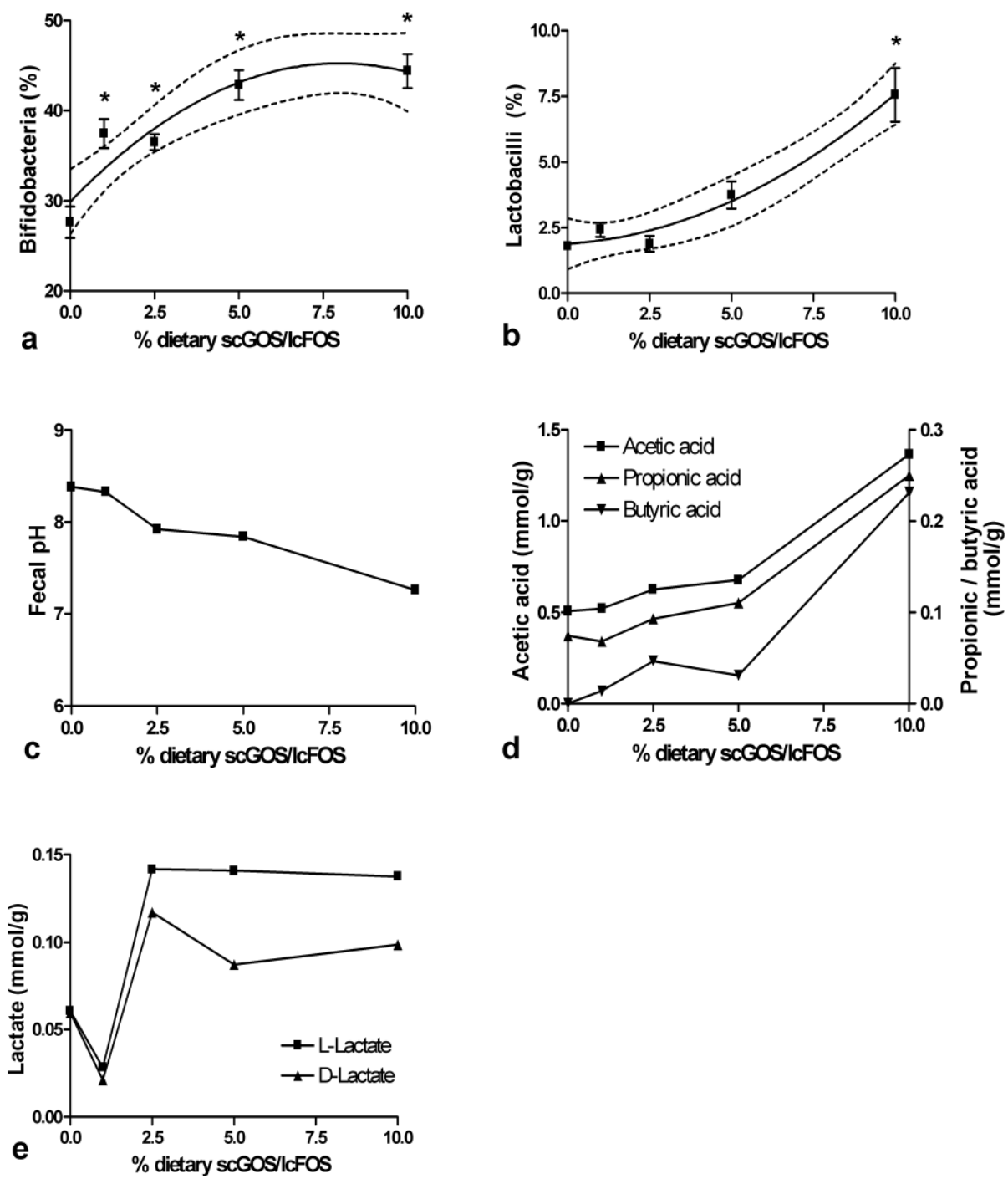

Figure 3. Prebiotic effects of different doses of scGOS/lcFOS. The percentage of (a) bifidobacteria and (b) lactobacilli relative to total bacterial counts in pooled samples after scGOS/lcFOS supplementation, measured in triplicate. Quadratic regression was performed and the optimal fitting curves are plotted in the graphs including $95 \% \mathrm{Cl}$ 's. * Indicates $\mathrm{p}<0.05$ compared to the control group, ANOVA and post hoc Dunnett's test; error bars indicate SEM. (c) pH of the fecal samples. (d) SCFA content of the fecal samples. (e) Lactate content of the fecal samples. Data in (c), (d) and (e) were measured once due to shortage of sample, therefore no statistics were performed. 

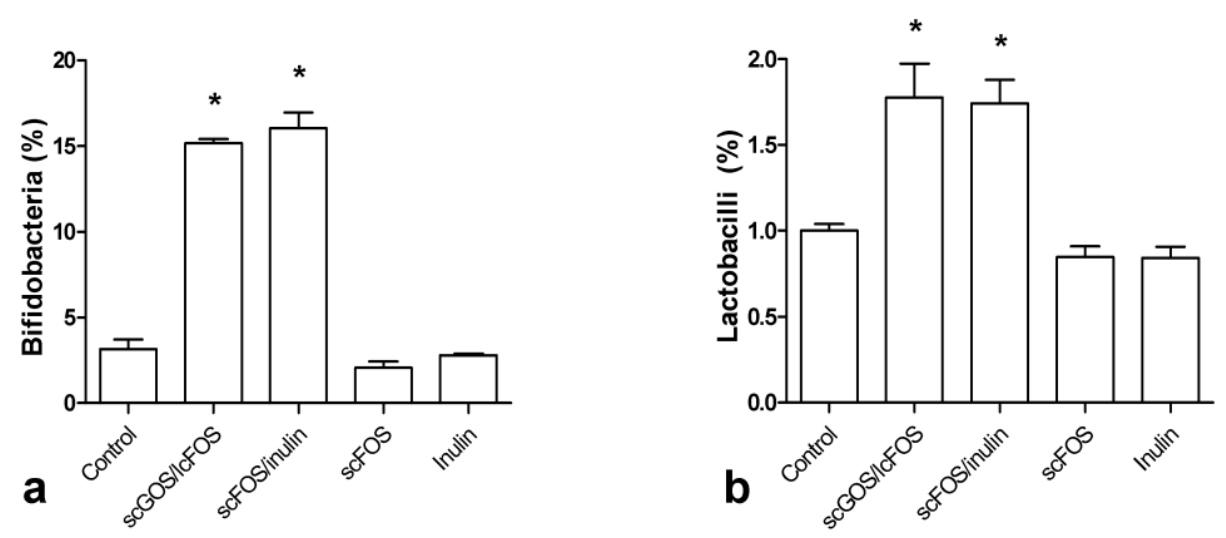

Figure 4. Effects of different oligosaccharide preparations on the proportions of bifidobacteria and lactobacilli in the intestinal microbiota. The proportion of (a) bifidobacteria and (b) lactobacilli in pooled colon samples relative to the total bacterial count are presented, measured in triplicate. All oligosaccharide preparations were supplemented at $2 \%$ (w/w of total diet) in the diets. * Indicates $\mathrm{p}<0.05$ compared to the control group; error bars indicate SEM.

\section{Discussion}

This study shows that dietary intervention with the prebiotic scGOS/lcFOS mixture enhances systemic adaptive immune responses in a murine vaccination model. This effect is reported for the first time, in combination with simultaneous measurements of parameters of the intestinal microbiota. The increased DTH responses after vaccination indicate that dietary supplementation with scGOS/lcFOS enhances parameters of Th1dependent immunity in vivo at relatively low doses. In our experience the magnitude of the immune-modulatory effect of scGOS/lcFOS at doses of 1 to $2.5 \%$ (w/w of total diet) varied between separate experiments, but the stimulatory effect on DTH responses after vaccination was consistently observed. In addition, this effect appears to be specific to scGOS/lcFOS, because three other oligosaccharide preparations did not modulate DTH responses.

scGOS/lcFOS supplementation had no effect on vaccine induced splenocyte proliferation, suggesting that the increase in DTH response is not caused by an increase in the general activation status of splenic vaccine-specific T-cells. Additionally, scGOS/lcFOS supplementation had no effects on vaccine-specific IgG, IgG1 and IgG2a antibody responses. The current model might not be sufficiently sensitive to detect changes in antibody concentrations, related to the high intrinsic biological variation in 
this parameter, or the immune-modulatory mechanism of scGOS/lcFOS supplementation might specifically affect cellular but not humoral immune responses.

Immune-modulatory effects of prebiotics have been shown before by others, but most studies have focused on the mucosal part of the immune system. Animal studies have shown effects on Peyer's patch (PP) lymphocyte populations and cytokine production $(11,12)$, increased PP and intestinal IgA production (13), as well as shifts in cytokine production in mucosal lymphocyte cultures (14). Systemic effects have been reported concerning total IgE serum concentrations (14), natural killer cell activity and phagocytic activity (34), tumor occurrence (35-39) and murine mortality rates after systemic infections with Listeria monocytogenes and Salmonella typhimurium (38). However, unlike the present study, these reports on systemic effects do not provide direct evidence for modulation of systemic adaptive immune responses.

It has been described that specific dietary probiotics enhance DTH responses in mice and rats (10). In this study, dietary scGOS/lcFOS is shown to cause a similar effect on DTH responses and to modulate the intestinal microbiota and related biochemical parameters (i.e. SCFAs, lactate and $\mathrm{pH}$ ), suggesting a common mechanism that involves intestinal bacteria. However, scFOS/inulin supplementation at $2 \%(\mathrm{w} / \mathrm{w}$ of total diet) resulted in a prebiotic effect, but did not affect immune function. In addition, the scGOS/lcFOS dose-response relationships were not identical for the DTH and the microbiological parameters. Therefore, the hypothesis that oligosaccharides modulate the adaptive immune system due to general bifidogenic and/or lactogenic effects and/or to changes in related biochemical parameters, should be reconsidered. It is possible that the stimulation of a specific bacterial (sub)strain by scGOS/lcFOS, but not by scFOS/inulin, causes the increase in DTH responses. Alternatively, oligosaccharides may also cause direct effects on the immune system: a small proportion of FOS is absorbed and secreted in the urine (40), intraperitoneally administered short-chain oligosaccharides were shown to affect airway eosinophilia in rats (41), and specific forms of inulin have been described to activate the alternative complement pathway and act as adjuvants upon injection or co-injection with antigen (42-45).

In conclusion, this report shows that dietary scGOS/lcFOS (ratio 9:1) supplementation induced systemic immune modulation of Th1-dependent adaptive responses in mice. Other FOS and inulin preparations that were tested in this study did not show immune-modulatory effects, which suggests that these effects are specific for the scGOS/lcFOS mixture. scGOS/lcFOS supplementation increased proportions of intestinal bifidobacteria and lactobacilli in a dose-dependent manner, similar to effects 
that have been described in human infants (20-23). However, as the results indicate from this study, such general prebiotic effects are not sufficient in all cases to induce modulation of Th1-related responses. Therefore, immune-modulatory effects of scGOS/lcFOS and other oligosaccharides need to be analyzed in humans. Enhancing systemic Th1 dependent adaptive immune responses would lead in theory to better immune responses against infections and prevention and/or inhibition of excessive Th2 responses, which occur for instance in allergies. Whether dietary scGOS/lcFOS prevents and/or inhibits infections and allergies in humans is currently being investigated in clinical studies.

\section{References}

1. Engfer, M.B., B. Stahl, B. Finke, G. Sawatzki, and H. Daniel, Human milk oligosaccharides are resistant to enzymatic hydrolysis in the upper gastrointestinal tract. Am J Clin Nutr, 2000. 71(6): p. 1589-96.

2. Harmsen, H.J., A.C. Wildeboer-Veloo, G.C. Raangs, A.A. Wagendorp, N. Klijn, J.G. Bindels, and G.W. Welling, Analysis of intestinal flora development in breast-fed and formula-fed infants by using molecular identification and detection methods. J Pediatr Gastroenterol Nutr, 2000. 30(1): p. 61-7.

3. Gibson, G.R. and M.B. Roberfroid, Dietary modulation of the human colonic microbiota: introducing the concept of prebiotics. J Nutr, 1995. 125(6): p. 1401-12.

4. Djouzi, Z. and C. Andrieux, Compared effects of three oligosaccharides on metabolism of intestinal microflora in rats inoculated with a human faecal flora. Br J Nutr, 1997. 78(2): p. 313-24.

5. Buddington, R.K., C.H. Williams, S.C. Chen, and S.A. Witherly, Dietary supplement of neosugar alters the fecal flora and decreases activities of some reductive enzymes in human subjects. Am J Clin Nutr, 1996. 63(5): p. 709-16.

6. Kruse, H.P., B. Kleessen, and M. Blaut, Effects of inulin on faecal bifidobacteria in human subjects. Br J Nutr, 1999. 82(5): p. 375-82.

7. Videla, S., J. Vilaseca, M. Antolin, A. Garcia-Lafuente, F. Guarner, E. Crespo, J. Casalots, A. Salas, and J.R. Malagelada, Dietary inulin improves distal colitis induced by dextran sodium sulfate in the rat. Am J Gastroenterol, 2001. 96(5): p. 1486-93.

8. Sghir, A., J.M. Chow, and R.I. Mackie, Continuous culture selection of bifidobacteria and lactobacilli from human faecal samples using fructooligosaccharide as selective substrate. J Appl Microbiol, 1998. 85(4): p. 769-77.

9. Macfarlane, G.T. and J.H. Cummings, Probiotics, infection and immunity. Curr Opin Infect Dis, 2002. 15(5): p. 501-6.

10. de Waard, R., E. Claassen, G.C. Bokken, B. Buiting, J. Garssen, and J.G. Vos, Enhanced immunological memory responses to Listeria monocytogenes in rodents, as measured by delayed-type hypersensitivity (DTH), adoptive transfer of DTH, and protective immunity, following Lactobacillus casei Shirota ingestion. Clin Diagn Lab Immunol, 2003. 10(1): p. 59-65.

11. Manhart, N., A. Spittler, H. Bergmeister, M. Mittlbock, and E. Roth, Influence of fructooligosaccharides on Peyer's patch lymphocyte numbers in healthy and endotoxemic mice. Nutrition, 2003. 19(7-8): p. 657-6o. 
12. Roller, M., G. Rechkemmer, and B. Watzl, Prebiotic inulin enriched with oligofructose in combination with the probiotics Lactobacillus rhamnosus and Bifidobacterium lactis modulates intestinal immune functions in rats. J Nutr, 2004. 134(1): p. 153-6.

13. Nakamura, Y., S. Nosaka, M. Suzuki, S. Nagafuchi, T. Takahashi, T. Yajima, N. Takenouchi-Ohkubo, T. Iwase, and I. Moro, Dietary fructooligosaccharides up-regulate immunoglobulin A response and polymeric immunoglobulin receptor expression in intestines of infant mice. Clin Exp Immunol, 2004. 137(1): p. 52-8.

14. Nagura, T., S. Hachimura, M. Hashiguchi, Y. Ueda, T. Kanno, H. Kikuchi, K. Sayama, and S. Kaminogawa, Suppressive effect of dietary raffinose on T-helper 2 cell-mediated immunity. Br J Nutr, 2002. 88(4): p. 421-6.

15. Swanson, K.S., C.M. Grieshop, E.A. Flickinger, N.R. Merchen, and G.C. Fahey, Jr., Effects of supplemental fructooligosaccharides and mannanoligosaccharides on colonic microbial populations, immune function and fecal odor components in the canine. J Nutr, 2002. 132(6 Suppl 2): p. 1717S-9S.

16. Albers, R., J.M. Antoine, R. Bourdet-Sicard, P.C. Calder, M. Gleeson, B. Lesourd, S. Samartin, I.R. Sanderson, J. Van Loo, F.W. Vas Dias, and B. Watzl, Markers to measure immunomodulation in human nutrition intervention studies. Br J Nutr, 2005. 94(3): p. 452-81.

17. Cummings, J.H., J.M. Antoine, F. Azpiroz, R. Bourdet-Sicard, P. Brandtzaeg, P.C. Calder, G.R. Gibson, F. Guarner, E. Isolauri, D. Pannemans, C. Shortt, S. Tuijtelaars, and B. Watzl, PASSCLAIM(1)-Gut health and immunity. Eur J Nutr, 2004. 43 Suppl 2: p. Il118-Il173.

18. Stahl, B., S. Thurl, J. Zeng, M. Karas, F. Hillenkamp, M. Steup, and G. Sawatzki, Oligosaccharides from human milk as revealed by matrix-assisted laser desorption/ionization mass spectrometry. Anal Biochem, 1994. 223(2): p. 218-26.

19. Finke, B., B. Stahl, A. Pfenninger, M. Karas, H. Daniel, and G. Sawatzki, Analysis of high-molecularweight oligosaccharides from human milk by liquid chromatography and MALDI-MS. Anal Chem, 1999. 71(17): p. 3755-62.

20. Boehm, G., S. Fanaro, J. Jelinek, B. Stahl, and A. Marini, Prebiotic concept for infant nutrition. Acta Paediatr Suppl, 2003. 91(441): p. 64-7.

21. Moro, G.E., F. Mosca, V. Miniello, S. Fanaro, J. Jelinek, B. Stahl, and G. Boehm, Effects of a new mixture of prebiotics on faecal flora and stools in term infants. Acta Paediatr Suppl, 2003. 91(441): p. 77-9.

22. Schmelzle, H., S. Wirth, H. Skopnik, M. Radke, J. Knol, H.M. Bockler, A. Bronstrup, J. Wells, and C. Fusch, Randomized double-blind study of the nutritional efficacy and bifidogenicity of a new infant formula containing partially hydrolyzed protein, a high beta-palmitic acid level, and nondigestible oligosaccharides. J Pediatr Gastroenterol Nutr, 2003. 36(3): p. 343-51.

23. Boehm, G., J. Jelinek, J. Knol, L. M'Rabet, B. Stahl, P. Vos, and J. Garssen, Prebiotics and Immune Responses. J Pediatr Gastroenterol Nutr, 2004 - 39: p. S772-S773.

24. Newburg, D.S. and S.H. Neubauer, Carbohydrates in Milks: Analysis, Quantities, and Significance, in Handbook of Milk Composition, R.G. Jensen, Editor. 1995, Academic Press: San Diego. p. 273-349.

25. Foda, M.I., T. Kawashima, S. Nakamura, M. Kobayashi, and T. Oku, Composition of milk obtained from unmassaged versus massaged breasts of lactating mothers. J Pediatr Gastroenterol Nutr, 2004. 38(5): p. 484-7.

26. Langlands, S.J., M.J. Hopkins, N. Coleman, and J.H. Cummings, Prebiotic carbohydrates modify the mucosa associated microflora of the human large bowel. Gut, 2004. 53(11): p. 1610-6.

27. Gibson, G.R., E.R. Beatty, X. Wang, and J.H. Cummings, Selective stimulation of bifidobacteria in the human colon by oligofructose and inulin. Gastroenterology, 1995. 108(4): p. 975-82.

28. Haarman, M. and J. Knol, Quantitative real-time PCR assays to identify and quantify fecal Bifidobacterium species in infants receiving a prebiotic infant formula. Appl Environ Microbiol, 2005. 71(5): p. 2318-24. 
29. Haarman, M. and J. Knol, Quantitative real-time PCR analysis of fecal Lactobacillus species in infants receiving a prebiotic infant formula. Appl Environ Microbiol, 2006. 72(4): p. 2359-65.

30. Nadkarni, M.A., F.E. Martin, N.A. Jacques, and N. Hunter, Determination of bacterial load by realtime PCR using a broad-range (universal) probe and primers set. Microbiology, 2002. 148(Pt 1): $\mathrm{p}$. 257-66.

31. Liu, W. and D.A. Saint, A new quantitative method of real time reverse transcription polymerase chain reaction assay based on simulation of polymerase chain reaction kinetics. Anal Biochem, 2002. 302(1): p. 52-9.

32. Snapper, C.M. and W.E. Paul, B cell stimulatory factor-1 (interleukin 4) prepares resting murine B cells to secrete IgG1 upon subsequent stimulation with bacterial lipopolysaccharide. J Immunol, 1987. 139(1): p. 10-7.

33. Berton, M.T., J.W. Uhr, and E.S. Vitetta, Synthesis of germ-line gamma 1 immunoglobulin heavychain transcripts in resting $B$ cells: induction by interleukin 4 and inhibition by interferon gamma. Proc Natl Acad Sci U S A, 1989. 86(8): p. 2829-33.

34. Kelly-Quagliana, K.A., P.D. Nelson, and R.K. Buddington, Dietary oligofructose and inulin modulate immune functions in mice. Nutr Res, 2003. 23: p. 257-267.

35. Taper, H.S., C. Lemort, and M.B. Roberfroid, Inhibition effect of dietary inulin and oligofructose on the growth of transplantable mouse tumor. Anticancer Res, 1998. 18(6A): p. 4123-6.

36. Pierre, F., P. Perrin, E. Bassonga, F. Bornet, K. Meflah, and J. Menanteau, T cell status influences colon tumor occurrence in min mice fed short chain fructo-oligosaccharides as a diet supplement. Carcinogenesis, 1999. 20(10): p. 1953-6.

37. Pierre, F., P. Perrin, M. Champ, F. Bornet, K. Meflah, and J. Menanteau, Short-chain fructooligosaccharides reduce the occurrence of colon tumors and develop gut-associated lymphoid tissue in Min mice. Cancer Res, 1997. 57(2): p. 225-8.

38. Buddington, K.K., J.B. Donahoo, and R.K. Buddington, Dietary oligofructose and inulin protect mice from enteric and systemic pathogens and tumor inducers. J Nutr, 2002. 132(3): p. 472-7.

39. Reddy, B.S., Possible mechanisms by which pro- and prebiotics influence colon carcinogenesis and tumor growth. J Nutr, 1999. 129(7 Suppl): p. 1478S-82S.

40. Molis, C., B. Flourie, F. Ouarne, M.F. Gailing, S. Lartigue, A. Guibert, F. Bornet, and J.P. Galmiche, Digestion, excretion, and energy value of fructooligosaccharides in healthy humans. Am J Clin Nutr, 1996. 64(3): p. 324-8.

41. Sonoyama, K., H. Watanabe, J. Watanabe, N. Yamaguchi, A. Yamashita, H. Hashimoto, E. Kishino, K. Fujita, M. Okada, S. Mori, S. Kitahata, and J. Kawabata, Allergic airway eosinophilia is suppressed in ovalbumin-sensitized Brown Norway rats fed raffinose and alpha-linked galactooligosaccharide. J Nutr, 2005. 135(3): p. 538-43.

42. Snyderman, R. and M.C. Pike, Interaction of complex polysaccharides with the complement system: effect of calcium depletion on terminal component consumption. Infect Immun, 1975. 11(2): p. 273-9.

43. Cooper, P.D., Vaccine adjuvants based on gamma inulin. Pharm Biotechnol, 1995. 6: p. 559-80.

44. Cooper, P.D. and E.J. Steele, The adjuvanticity of gamma inulin. Immunol Cell Biol, 1988. 66 ( Pt 5-6): p. 345-52.

45. Chien, C.C., Anti-inulin [beta-(2 leads to 1)-linked polyfructose] and anti-grass levan [beta-(2 leads to 6)-linked polyfructose] antibody response in mice. Infect Immun, 1980. 27(3): p. 746-55. 


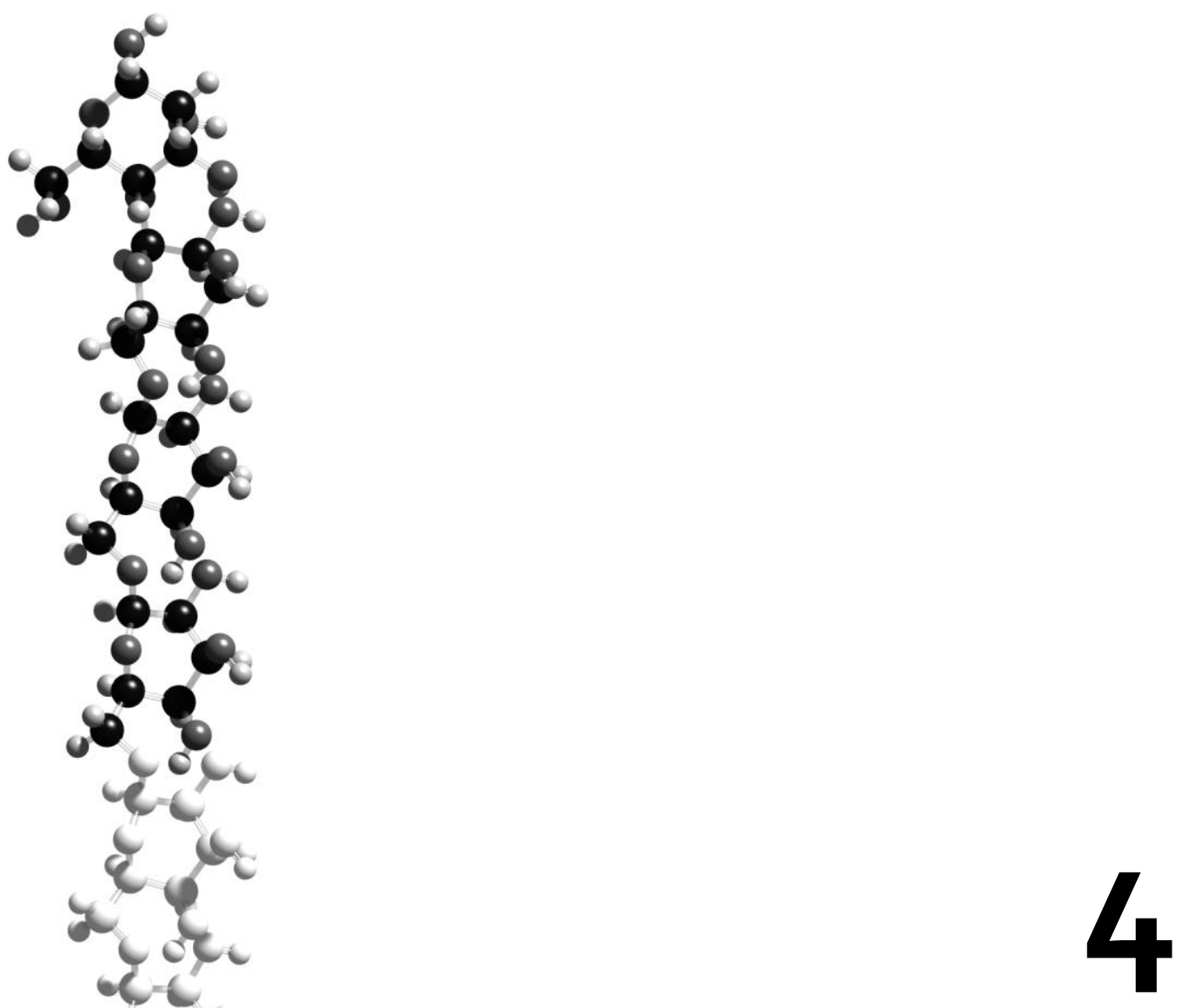

\section{Dietary supplementation of neutral and acidic oligosaccharides enhances} Th1-dependent vaccination responses in mice

A.P. Vos

M. Haarman J.W.H. van Ginkel

J. Knol

J. Garssen

B. Stahl

G. Boehm

L. M'Rabet 


\section{Abstract}

Immune-modulatory effects of oligosaccharide preparations that resemble chemical and functional aspects of human milk oligosaccharides (HMOS) were studied for the development of new concepts in infant nutrition. A dose range of 1-5\% (w/w) dietary pectin-derived acidic oligosaccharides (pAOS) was tested in a murine influenza vaccination model. In addition, combinations of pAOS and a 9:1 mixture of short-chain galactooligosaccharides and long-chain fructooligosaccharides (scGOS/lcFOS; Immunofortis) were tested at a fixed total dietary dose of $2 \%(w / w)$.

It was found that pAOS significantly enhanced vaccine-specific delayed-type hypersensitivity (DTH) responses in a dose-dependent manner. This was accompanied by a reduction in T-helper2 (Th2) cytokine production by splenocytes in vitro. Overall, this indicates that the systemic immune response to the vaccine was Th1-skewed by the dietary intervention. Combinations of scGOS/lcFOS and pAOS were more effective in enhancing DTH responses than either of the oligosaccharides alone, suggesting interaction effects between these agents. Similar to effects in infants, supplementation of the murine diets with scGOS/lcFOS and combinations of scGOS/lcFOS and pAOS for six weeks enhanced the proportion of fecal bifidobacteria and lactobacilli, but pAOS alone did not.

In conclusion, these data indicate that scGOS/lcFOS and pAOS enhance systemic Th1dependent immune responses in a murine vaccination model. As Th1-responses are weak in early life in humans, this might suggest that application of these oligosaccharides in infant formulas will be beneficial for the development of the infant's immune system.

\section{Introduction}

Human milk provides an infant with more than just nutritional value. Among other functions, it also provides immunological protection through constituents such as antibodies, complement, lysozyme, lactoferrin and maternal leukocytes (1). Human milk oligosaccharides (HMOS) have been shown to stimulate specific intestinal microbiota (2), to block pathogen adhesion sites in the gut and/or to act as soluble pathogen receptor analogs (3-6). This provides the infant with a non-specific mode of protection against invading pathogens in the gastrointestinal tract. Recently, Eiwegger 
et al. (7) have shown that HMOS, in particular the acidic fraction, affects the expression of $\mathrm{T}$ cell activation markers as well as cytokine production in cord blood cells in vitro. These findings suggest a direct immune-modulatory effect of HMOS and might implicate an additional mechanism of immunological protection provided by human milk.

For situations in which breast-feeding is not possible, infant nutrition should provide the best alternative care. Human milk contains a mixture of neutral and acidic oligosaccharides that features a complex structural diversity (8). As this diversity of complex structures is not available, a functional analogue was developed for infant nutrition. In a first step, a mixture of neutral short-chain galactooligosaccharides (scGOS) and long-chain fructooligosaccharides (lcFOS) in a 9:1 ratio (scGOS/lcFOS; Immunofortis) was designed to resemble the molecular size distribution and functional aspects of the neutral fraction of HMOS (9-11). Recently, this concept has been extended by the addition of acidic oligosaccharides derived from pectin (pAOS), to mimic the balance between neutral and acidic HMOS (12). Similar to the described antiadhesive properties of HMOS (3-5), Kastner et al. (13) have shown inhibitory effects in vitro of acidic, galacturonic acid containing oligosaccharides from carrot soup on the adhesion of various pathogens to Hep-2 cells and human intestinal mucosa.

In previous experiments (chapter 3), stimulatory effects of scGOS/lcFOS were shown on systemic immune responses in a murine vaccination model. This information, combined with the reported in vitro immune-modulatory effects of acidic HMOS (7), has led us to hypothesize that pAOS could induce immune-modulatory effects in vivo. This hypothesis was tested in a murine vaccination model, using galacturonic acid containing pAOS characterized by a degree of methyl esterification of $>40 \%$ and an amount of $\Delta 4,5$-unsaturated galacturonic acid at the non-reducing terminus of $>35 \%$. Additionally, pAOS were applied in combination with scGOS/lcFOS, to test for potential interaction of the respective immune-modulatory effects. In vivo and ex vivo immunological parameters were analyzed, as well as effects on the intestinal microbiota by measuring the percentage of fecal bifidobacteria and lactobacilli relative to the total bacterial counts.

\section{Materials and Methods}

\section{Mice}

Female, 6-week-old C57Bl/6JOlaHsd mice were obtained from Harlan (Horst, The Netherlands) and kept under normal conditions, with a 12-h dark and light cycle and 
access to food and water ad libitum. All experiments were approved by an independent animal experiments committee (DEC Consult, Bilthoven, The Netherlands).

\section{Diets}

All animals received semi-purified AIN-93G-based diets (Research Diet Services, Wijk

bij Duurstede, The Netherlands). All supplemented oligosaccharide products were exchanged for the same amount of total carbohydrates. This approach resulted in a comparable overall carbohydrate composition in different diets, to ensure that the gut flora was minimally influenced by differences between control and test diets in parameters such as gut passage time and fluid retention. The oligosaccharides were mixed into the AIN-93G diet and pressed into pellets. Deviations from the AIN-93G specification are specified in Table 1 for all supplemented diets. pAOS are produced from pectin (Südzucker AG, Mannheim, Germany), with a degree of polymerization (dp) of 1-20. It consists of approximately 75\% galacturonic acid oligomers, $10 \%$ monomers and $15 \%$ of moisture and ash. scGOS/lcFOS is a spray-dried powder of scGOS (Vivinal GOS, Borculo Domo, Zwolle, The Netherlands; mainly dp 2-6) and lcFOS with a high degree of polymerization (Raftiline HP, Orafti, Wijchen, The Netherlands; $d p>10$ ) in a 9:1 ratio. Maltodextrin was used as carrier for the spraydrying process. scGOS/lcFOS consists of approximately 51\% scGOS and lcFOS oligosaccharides, 19\% maltodextrin, $16 \%$ lactose, and $14 \%$ glucose.

Table 1. Composition of the diets

\begin{tabular}{|c|c|c|c|c|c|}
\hline \multirow[b]{2}{*}{ Ingredients (g/kg) } & \multirow{2}{*}{$\begin{array}{l}\text { Control diets } \\
\text { (AIN93G) }\end{array}$} & \multicolumn{3}{|c|}{ pAOS dose-response diets } & \multirow{2}{*}{$\begin{array}{c}\text { Diets containing } 2 \% \\
\text { oligosaccharide } \\
\text { preparations }\end{array}$} \\
\hline & & $1 \%$ & $2.5 \%$ & $5 \%$ & \\
\hline Cornstarch & $397 \cdot 5$ & 391.6 & 382.9 & 368.2 & 385.8 \\
\hline Dextrinized cornstarch & 132.0 & 130.1 & 127.1 & 122.3 & 128.1 \\
\hline Sucrose & 100.0 & 98.5 & 96.3 & 92.6 & 97.1 \\
\hline Cellulose & 50.0 & $49 \cdot 3$ & 48.2 & 46.3 & 48.5 \\
\hline Added carbohydrates & & $10^{a}$ & $25^{a}$ & $50^{a}$ & $20^{b}$ \\
\hline
\end{tabular}

${ }^{\mathrm{a}} \mathrm{pAOS} ;{ }^{\mathrm{b}} \mathrm{scGOS/lcFOS,} \mathrm{pAOS}$ or a combination of both; see text for details on the added carbohydrates 


\section{Vaccination protocol and DTH response}

The vaccination protocol has been described previously (chapter 3), and was shown to induce sub-maximal immune responses that allow the detection of stimulatory as well as inhibitory immune-modulatory effects. In short, the mice received primary and booster vaccinations, consisting of a subcutaneous (sc) injection of a 1:1 mix of vaccine solution (100x diluted Influvac 2002/2003 in PBS, Solvay Pharmaceuticals, Weesp, The Netherlands) and adjuvant (Stimune; Cedi-diagnostics, Lelystad, The Netherlands). Sham vaccination groups received injections of a 1:1 mix of PBS and adjuvant. Booster vaccinations were given 21 days after the primary vaccination. Delayed-type hypersensitivity responses (DTH) reactions were induced 9 days after booster vaccination, by sc injection of $25 \mu \mathrm{L}$ Influvac into the ear pinnae of both ears. Ear thickness was measured in duplicate before antigen challenge, as well as 24 hours afterwards, with a digital micrometer (Mitutoyo Digimatic 293561, Veenendaal, The Netherlands). The DTH response was calculated by subtracting the basal ear thickness from the value at $24 \mathrm{~h}$ after challenge. The experiments ended 10 days after booster vaccination.

\section{Dietary intervention experiments}

A dose-response experiment with pAOS supplementation was performed (experiment 1 ), using doses of $1 \%, 2.5 \%$ and $5 \%(w / w)$ in the diets ( $n=10$ in all groups). In addition, a combination experiment was carried out by using scGOS/lcFOS, pAOS and combinations thereof (experiment 2). All test groups in experiment 2 featured a total supplementation dose of $2 \%(\mathrm{w} / \mathrm{w})$ of the oligosaccharide preparations; (I) $2 \%$ scGOS/lcFOS ( $\mathrm{n}=10)$, (II) $2 \%$ pAOS (n=10), (III) $1 \%$ scGOS/lcFOS + 1\% pAOS ( $\mathrm{n}=8)$ and (IV) $1.8 \%$ scGOS/lcFOS $+0.2 \%$ pAOS $(n=10)$ were tested. Expressed as the net oligosaccharide ratios between the scGOS/lcFOS and pAOS oligosaccharides, group III contains scGOS/lcFOS:pAOS in a 2:3 mix and group IV contains scGOS/lcFOS:pAOS in a 6:1 mix, because of the differences in specific oligosaccharide content of the different oligosaccharide preparations.

All mice were given control AIN-93G diets upon arrival at the animal facility for a minimum of 5 days. The dietary interventions in supplementation groups started 14 days before the primary vaccination and lasted until the last day of the experiment, 31 days after the primary vaccination. In both experiments, a control group was included $(n=10)$ that was vaccinated normally and received the control diet (unsupplemented AIN-93G). Sham vaccination groups $(n=3)$ were also included in both experiments. These groups were not used for statistical calculations or comparisons, but served 
solely to demonstrate the specificity of vaccine-induced responses.

\section{Splenocyte proliferation assay}

Splenocytes were isolated by gently pressing spleen tissue through nylon mesh filters (Falcon cell strainer; Becton Dickinson, Alphen a/d Rijn, the Netherlands). After erythrocyte lysis using an ammonium-chloride buffer, cells were counted and diluted to the appropriate concentrations. Cultures were stimulated with Influvac vaccine at a predetermined optimal concentration, and proliferation was measured by tritiated thymidine incorporation. In short, $1^{*} 10^{6}$ cells were distributed in 96-well plates in triplicate in RMPI-1640 medium (Invitrogen, Merelbeke, Belgium) supplemented with $10 \%$ fetal calf serum (FCS), $100 \mathrm{U} / \mathrm{ml}$ penicillin and $100 \mu \mathrm{g} / \mathrm{mL}$ streptomycin. Cells were stimulated with control medium or with $0.1 \mu \mathrm{g} / \mathrm{mL}$ Influvac vaccine solution. After four days of culture, $0.4 \mu \mathrm{Ci} /$ well tritiated thymidine was added, and 24 hours later cells were harvested (Filtermate, Perkin Elmer) and counted in a scintillation counter (Wallac Microbeta, Perkin Elmer). Data are presented as the difference in counts per minute (cpm) between control medium and Influvac-stimulated wells.

Splenocytes that were not used for initiating cell cultures were kept on ice and taken up in RPMI-1640 medium with $25 \%$ FCS and $12.5 \%$ DMSO at $1^{*} 10^{7}$ cells $/ \mathrm{ml}$. Cell suspensions were dispensed in cryovials (Greiner, Alphen aan de Rijn, The Netherlands), which were placed in cryocontainers (Nalgene, Omnilabo, Breda, The Netherlands) and stored overnight at $-80{ }^{\circ} \mathrm{C}$. Samples were stored in liquid nitrogen until further analysis.

\section{Ex vivo cytokine production assay}

Splenocytes cultures were set up for the measurement of ex vivo cytokine production, in parallel with the cultures for proliferation (described above), using the identical procedure. Supernatants were harvested after 5 days of culture and stored at $-80{ }^{\circ} \mathrm{C}$ until further analysis. The cytokines interferon (IFN) $\gamma$, interleukin (IL) 2, IL-4, IL-5 and IL-10 were measured by using a multiplex bead array according to the manufacturer's instructions (x-plex reagents, BioRad, Veenendaal, The Netherlands) on a Bio-Plex workstation (Bio-Rad). Results are shown as the difference in cytokine concentrations between stimulated and unstimulated wells, with the exception of the IL- 4 values, as all unstimulated wells showed IL-4 levels that were below the detection limit. The value of the detection limit was used in the calculation of group statistics in the case of undetectable cytokine concentrations. 


\section{Flow cytometric analysis of splenocyte populations}

For flow cytometric analysis, splenocyte suspensions were thawed, washed and taken up in cold PBS with 1\% FCS. Cells were incubated with fluorescently labeled antibodies for $30 \mathrm{~min}$ on ice. After washing, cells were analyzed on an Epics XL flow cytometer (Beckman Coulter, Mijdrecht, The Netherlands). Propidium iodide staining and forward- and side-scatter profile was used to identify intact living cells. The following monoclonal rat-anti mouse antibodies were used: CD3-FITC (Cat. Nr. IM2768), CD8-PE (Cat. Nr. IM2780), CD19-FITC (Cat. Nr. IM3233), CD25-PE (Cat. Nr. IM 2795), NK1.1PE (Cat. Nr. IM2904; all from Beckman Coulter) and CD4-PE-CY5 (Cat. Nr. 553654, Becton Dickinson).

\section{Fecal and colon content sample isolation}

In experiment 2, fecal samples were collected from the cages from a 12-h period at the end of study and were stored at $-20{ }^{\circ} \mathrm{C}$. Samples of the colon content were isolated directly after the animals were sacrificed and stored at $-20^{\circ} \mathrm{C}$.

\section{Quantitative real time PCR of the genus Bifidobacterium and Lactobacillus}

For the relative quantification of the genera Bifidobacterium and Lactobacillus in relation to the total bacterial load, a duplex 5' nuclease quantitative real-time PCR (qPCR) assay was used as previously published $(14,15)$.

Briefly, thawed samples were pooled per experimental group (using a fixed weight of sample per animal) and 10x diluted in saline (w/v). Different primers and probes for the genus Bifidobacterium or Lactobacillus in combination with primers and probes for the total bacterial load (16) were used in a temperature profile consisting of $2 \mathrm{~min}$ at $50^{\circ} \mathrm{C}, 10 \mathrm{~min}$ at $95^{\circ} \mathrm{C}$, followed by 45 cycles of $15 \mathrm{~s}$ at $95^{\circ} \mathrm{C}$ and $60^{\circ} \mathrm{C}$ for $1 \mathrm{~min}$, run on ABI Prism 7700 PCR equipment (Applied Biosystems, Nieuwerkerk a/d IJssel, The Netherlands).

Thereafter, the relative amounts of the genus Bifidobacterium or Lactobacillus in the samples were calculated with respect to the total bacterial load according to Liu et al. (17), and expressed in percentages. All samples were measured in triplicate.

\section{Statistical methods}

Statistical calculations were performed in SPSS version 12.0.1 (SPSS inc., Chicago, IL, USA); regression analysis and graph plotting was performed in Graphpad Prism 4.03 software (San Diego, CA, USA). Dose-response relationships in experiment 1 were 
analyzed using linear regression, testing the slopes of the regression lines for a deviation from zero. Statistical differences between groups in experiment 2 were analyzed by ANOVA and post hoc Bonferroni's test. Differences in microbiological parameters at different time points were analyzed by using Student's T-test, using Bonferroni-adapted p-values to correct for multiple comparisons. p-values $<0.05$ were considered to be statistically significant.

\section{Results}

\section{Experiment 1}

pAOS supplementation at $1-5 \%$ in the diet was well tolerated by the mice; no adverse effects were noted during the experiment. Feed uptake and body weights were slightly higher in pAOS supplemented mice compared with control mice (Table 2). Regression analysis showed a significant dose-response relationship for both parameters.

Mice that received pAOS supplementation showed significantly enhanced, dosedependent DTH responses after vaccination in comparison with control mice (Fig. 1a). Background responses after antigen challenge were low in the sham group $(4.8 \pm 8.2$

Table 2. Animal weights and feed intake

\begin{tabular}{|c|c|c|c|c|c|c|c|}
\hline \multirow[b]{2}{*}{ Experiment } & \multirow[b]{2}{*}{ Group } & \multicolumn{3}{|c|}{$\begin{array}{l}\text { Animal weight at the } \\
\text { end of the experiment }\end{array}$} & \multicolumn{3}{|c|}{$\begin{array}{l}\text { Weekly feed intake per } \\
\text { animal }\end{array}$} \\
\hline & & Mean & & SEM & Mean & & SEM \\
\hline \multirow{4}{*}{$\begin{array}{l}\text { pAOS dose-response } \\
\text { experiment }^{a}\end{array}$} & Control & 19.67 & & 0.25 & 16.99 & \multirow{4}{*}{ * } & 0.70 \\
\hline & $1 \%$ & 21.50 & & 0.43 & 17.90 & & 0.48 \\
\hline & $2.5 \%$ & 20.70 & * & 0.56 & 17.66 & & 0.72 \\
\hline & $5 \%$ & 22.36 & & 0.43 & 18.41 & & 0.67 \\
\hline \multirow{5}{*}{$\begin{array}{l}\text { Combination } \\
\text { experiment }^{\text {b }}\end{array}$} & Control & 19.67 & & 0.36 & 16.34 & & 0.40 \\
\hline & scGOS/lcFOS & 20.05 & & 0.35 & 16.58 & & 0.42 \\
\hline & pAOS & 19.89 & & 0.28 & 17.05 & & 0.49 \\
\hline & $1: 1$ & 19.43 & & 0.31 & 16.95 & & 0.41 \\
\hline & $1.8: 0.2$ & 20.48 & & 0.43 & 17.81 & & 0.49 \\
\hline
\end{tabular}

Animals were weighed weekly; data are shown from the last measurement. Feed intake was measured weekly per animal cage; the data are shown as the mean intake per animal per week. a Data were analyzed by linear regression analysis; * indicates a significant dose-response relationship $(p<0.05)$. bData were analyzed by ANOVA; no significant differences were observed. 

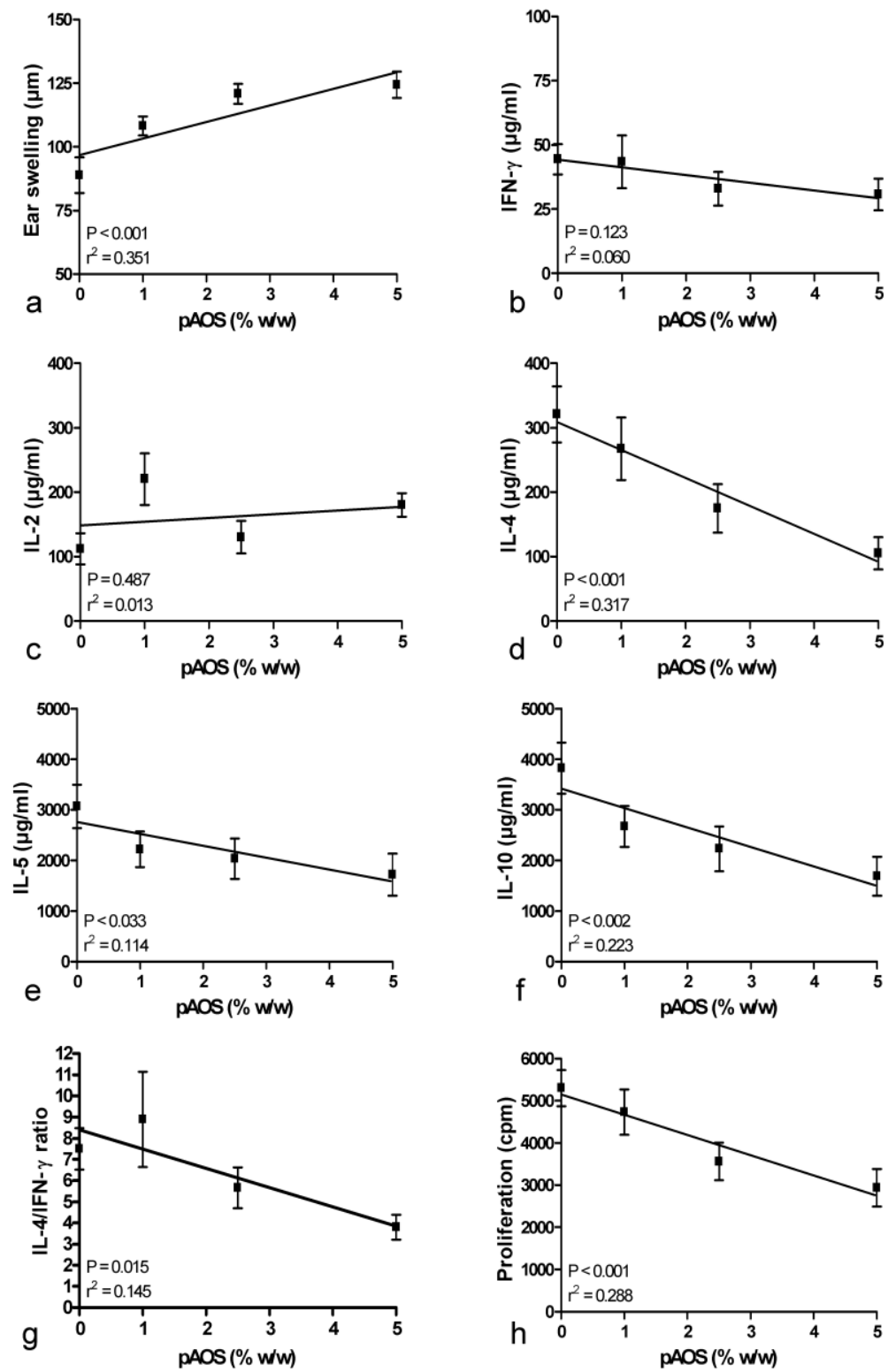

Figure 1. Dietary pAOS affect parameters of immune function in vivo and ex vivo. Supplementation doses of $1-5 \%$ pAOS were tested in a murine vaccination model as indicated on the $x$-axes. (a) At the end of the experiment, in vivo vaccine-specific DTH responses were analyzed by measuring ear swelling $24 \mathrm{~h}$ after antigen injection. (b-g) Splenocytes were isolated and cultured in vitro. After 5 days of antigen-specific stimulation, IFN$Y$ (b), IL-2 (c), IL-4 (d), IL-5 (e) and IL-10 (f) concentrations were measured in culture supernatants, the IL-4/IFN- $\gamma$ ratio in culture supernatants was calculated per animal and averaged per experimental group $(\mathrm{g})$ and $\mathrm{T}$ cell proliferation was measured using $3 \mathrm{H}$-thymidine incorporation (h). Data are represented as group means and error bars indicate SEM. 
$\mu \mathrm{m}$; mean \pm SEM) compared with the control group $(88.7 \pm 7.3 \mu \mathrm{m})$, demonstrating that the responses were vaccination-dependent secondary responses. DTH responses were $40.1 \%$ higher in the group that received the highest pAOS supplementation dose (124.3 $\pm 5.5 \mu \mathrm{m})$ than in control animals.

In addition, ex vivo cytokine production and proliferation by vaccine-stimulated splenocyte cultures were affected by dietary intervention with pAOS (Fig. 1b-h). No significant effects were observed on the production of Th1-type cytokines; IFN- $\gamma$ production showed a decreasing trend and IL-2 production was elevated in the $1 \%$ dose-group but not in other dose-groups, showing no clear dose-effect relationship. pAOS supplementation decreased the production of Th2-type cytokines significantly in a dose-dependent manner. IL-4 and IL-5 production were significantly reduced; the effect was most pronounced on IL-4 production, ranging from $320.9 \pm 43.8 \mathrm{pg} / \mathrm{ml}$ in the control group to $105.2 \pm 25.1 \mathrm{pg} / \mathrm{ml}$ in the highest dose group. The production of IL-10, a cytokine produced by Th2 cells as well as other cell types, was also dose dependently reduced. To further analyze the Th1/Th2 balance, the ratio of IL-4 and IFN- $\gamma$ production was calculated per animal and averaged per experimental group. Fig. $1 \mathrm{~g}$ shows that the IL-4/IFN- $\gamma$ ratio decreased dose-dependently with pAOS supplementation. In parallel to Th2-type cytokine production, antigen-specific proliferation was dose-dependently decreased as well (Fig. 1h).

\section{Experiment 2}

Supplementation with combinations of pAOS and scGOS/lcFOS strongly enhanced vaccine-specific DTH responses compared with control-fed animals; $1 \%$ scGOS/lcFOS + $1 \%$ pAOS (group III) showed a response of $201 \pm 11 \mu \mathrm{m}$, compared with $95 \pm 9 \mu \mathrm{m}$ in control animals. As in experiment 1, background responses were low in the sham group $(6.8 \pm 3.9 \mu \mathrm{m})$. Animals in group III exhibited significantly higher DTH responses than animals that received either scGOS/lcFOS or pAOS (groups I and II). In addition, a combination of scGOS/lcFOS with a small amount of pAOS in group IV (net scGOS/lcFOS:pAOS ratio 6:1) resulted in significantly higher DTH responses than supplementation with only scGOS/lcFOS in group I (Fig. 2a). There were no differences in feed uptake or animal weight in experiment 2 (Table 2).

Ex vivo proliferation responses in the control group from experiment 2 (5025 $\pm 823 \mathrm{cpm})$ were similar to those in experiment $1(5302 \pm 430 \mathrm{cpm})$. IL-4 production in the control group of experiment 2 was low $(38 \pm 22 \mathrm{pg} / \mathrm{ml})$ and IFN- $\gamma$ production was high $(847 \pm$ $396 \mathrm{pg} / \mathrm{ml}$ ) compared with experiment 1 . The intra-group variation in cytokine concentrations was high in experiment 2, and proliferation responses or cytokine 

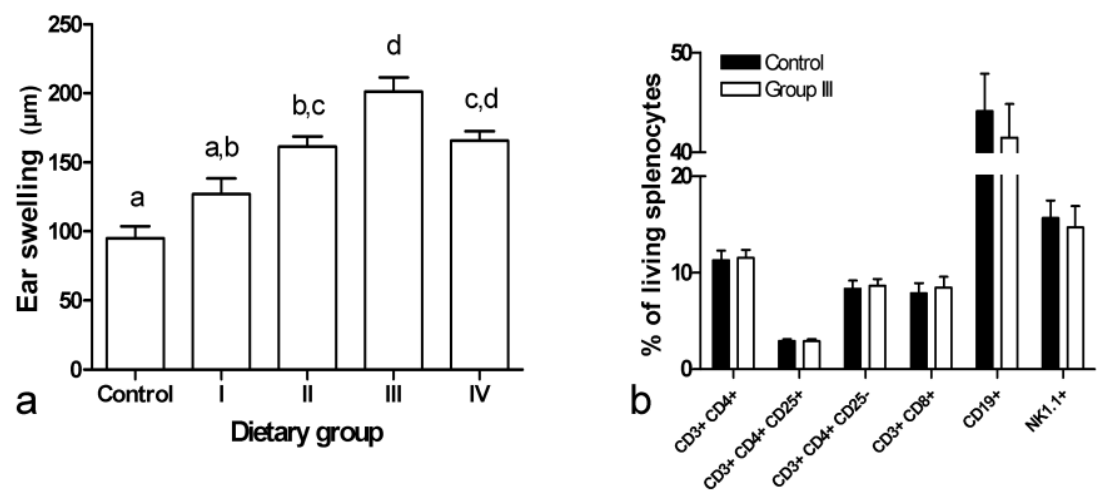

\section{Population}
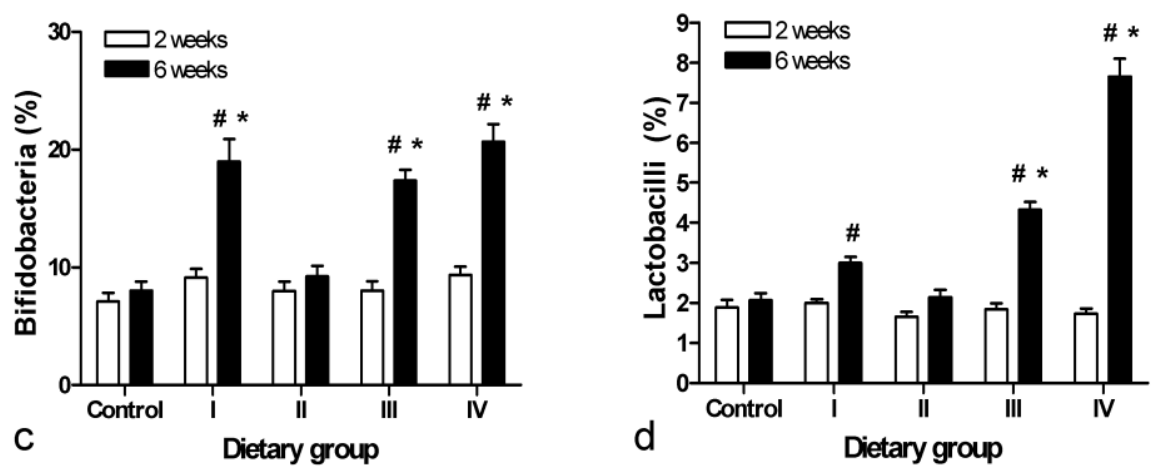

Figure 2. The effects of combinations dietary of scGOS/lcFOS, pAOS and combinations thereof on DTH responses and the intestinal microflora. Dietary supplementation of (I) $2 \%(\mathrm{w} / \mathrm{w}) \mathrm{scGOS} / \mathrm{lcFOS}$, (II) $2 \% \mathrm{pAOS}$, (III) $1 \% \mathrm{scGOS} / \mathrm{lcFOS}+1 \% \mathrm{pAOS}$ and (IV) $1.8 \% \mathrm{scGOS} / \mathrm{lcFOS}+0.2 \%$ pAOS (IV) was tested versus control diet (Control) in a murine vaccination model, as indicated on the $\mathrm{x}$-axis or in the legend. (a) At the end of the experiment, in vivo DTH responses were analyzed by measuring ear swelling $24 \mathrm{~h}$ after antigen injection. Statistical differences between groups are indicated in the graph. Groups that do not share an indicator are different at $p<0.05$. (b) Splenocyte populations from the control group and group III were analyzed by flow cytometry. The data is presented as the percentage of total living splenocytes. (c, d) Bifidobacteria (c) and lactobacilli (d) were measured in fecal samples as a proportion of the total bacterial load. The fecal samples were pooled per group and measured in triplicate. Samples were collected after 2 wk of supplementation (white bars) and after 6 wk of supplementation (black bars). * Indicates $p<0.05$ compared with the control group. \# Indicates $p<0.05$ between values at 2 and 6 wk of supplementation within a dietary group. Data in all graphs represent qroup means and error bars indicate SEM.

production did not differ significantly between experimental groups (data not shown).

To investigate whether the difference in DTH responsiveness between both groups was related to changes in systemic lymphocyte populations, splenocytes from control and group III animals from experiment 2 were analyzed by flow cytometry (Fig. 2b). T cell 
$\mathrm{CD}^{+}$and $\mathrm{CD}^{+}$subsets were measured, including analysis of $\mathrm{CD} 4{ }^{+} \mathrm{CD} 25^{+}$activated or regulatory $\mathrm{T}$ cells. $\mathrm{T}$ cell subsets did not differ significantly between control and group III animals. In addition, CD19+ B-cell and NK1.1+ natural killer cell populations did not differ significantly between the groups.

Freshly collected fecal samples from experiment 2 were analyzed from two time points; after $2 \mathrm{wk}$ and after $6 \mathrm{wk}$ of supplementation. The analyses of the relative amount of bifidobacteria and lactobacilli are shown in Fig. $2 \mathrm{c}$ and $2 \mathrm{~d}$, respectively. Exposure to pAOS as a single agent (group II) did not induce changes compared with control animals, whereas scGOS/lcFOS supplementation (group I) resulted in a significantly elevated percentage of bifidobacteria. Combinations of pAOS and scGOS/lcFOS resulted in significant relative increases in bifidobacteria as well as lactobacilli in groups III and IV. All supplemented groups, except group II, showed significantly elevated percentages of both bifidobacteria and lactobacilli in the samples taken after 6 wk, compared with the samples taken after $2 \mathrm{wk}$.

\section{Discussion}

The immune system at birth is not yet fully matured. Th1 responses are weak shortly after birth and tend to increase progressively with age, causing immune responses in early life to be polarized towards Th2 responses in mice and man (reviewed in 18). In addition to the well-known role that breast milk plays in providing passive protection via immunoglobulins and other factors, Pabst has hypothesized (19) that breast milk also plays an active role in the development of the infant's immune system. Pabst et al. (20) also provided some evidence of more effective Th1 responses in infants that had received breast milk, compared with infants that had received infant nutrition. In support of this view, there are multiple reports in literature showing lower incidences of infections (21-23) and atopy-related disorders (24-26) in breast-fed children compared with formula-fed children. These immunological effects could potentially be mediated by a myriad of factors in breast milk, precluding the incorporation of all relevant ingredients in an infant nutrition. A practical approach is to mimic breast milk functionally, by including ingredients that support the development of Th1 responses, for use in situations when breast-feeding is not possible.

In our murine model, pAOS induced a dose-dependent increase in DTH response after vaccination, a Th1-dependent in vivo parameter. Additionally, in vitro production of Th2 but not Th1-related cytokines was decreased dose-dependently. Overall, the in vivo 
and in vitro data indicate that dietary intervention with pAOS causes a shift in the Th1/Th2 balance in a murine systemic immune response towards Th1. The observed decrease in antigen specific proliferation might be related to the decreased IL-4 production, which is a factor that can strongly promote $\mathrm{T}$ cell division (27). pAOS containing diets increased feed uptake and animal weight dose-dependently for unknown reasons. Although it cannot be ruled out that this might have played a role in the immunological effects that were observed, this is not likely, as the second experiment showed significant immunological effects between groups without differences in animal weight or feed uptake.

The oligosaccharide concept for application in infant nutrition features a combination of scGOS/lcFOS and pAOS (12). Because it was shown in previous work that scGOS/lcFOS enhanced vaccine-specific DTH responses (chapter 3), combinations of scGOS/lcFOS and pAOS were also tested in this study using a fixed total supplementation dose in all groups. A combination of $1 \%$ scGOS/lcFOS and $1 \%$ pAOS resulted in higher DTH responses than either single preparation at a $2 \%$ dose. In addition, a combination of $1.8 \%$ scGOS/lcFOS and $0.2 \%$ pAOS resulted in significantly increased DTH responses compared with $2 \%$ scGOS/lcFOS, while $0.2 \%$ pAOS alone is not expected to affect the DTH response significantly based on an interpolation of Fig. $1 d$. Overall, combinations of scGOS/lcFOS and pAOS resulted in unexpected higher $\mathrm{DTH}$ responses than either of the single preparations, suggesting a synergistic interaction.

In contrast to experiment 1 , there were no significant effects on cytokine production or cell proliferation in experiment 2, which might be due to the relatively low levels of pAOS in experiment 2. This might indicate differential effects of the oligosaccharide preparations on DTH responses and ex vivo cytokine production. On the other hand, it is also possible that effects on cytokine productions were masked by the high intragroup variation in experiment 2 .

The mechanism of action of dietary oligosaccharides in causing enhanced DTH responses is not yet clear. Mucosal and systemic Th1-stimulating effects of dietary oligosaccharides were recently published by Mizubuchi et al. (28), using a very high dose $(20 \% \mathrm{w} / \mathrm{w})$ of isomaltooligosaccharides. Parallel effects of these oligosaccharides were observed on microbiota and immunological parameters. However, direct effects were also shown on cytokine production in a macrophage-like cell line, indicating that multiple mechanisms might play a causal role in the immune-modulatory effects. Eiwegger et al. (7) described that the acidic fraction of HMOS enhanced the expression of CD25 on CD4 $4^{+} \mathrm{T}$ cells, an activation marker that is also expressed constitutively on 
regulatory $\mathrm{T}$ cells. This prompted us to conduct a flow cytometric analysis of splenocyte populations in animals from the control group and group III. There were no differences between the groups for any of the analyzed $\mathrm{T}$ cell populations including $\mathrm{CD} 4^{+} \mathrm{CD} 25^{+}$cells, or in B- and NK-cell populations, suggesting that the observed immune-modulatory effects were not caused by phenotypic changes in major systemic lymphocyte or NK-cell populations.

In clinical studies, the inclusion of scGOS/lcFOS into infant nutrition resulted in a significant increase in the number of bifidobacteria and lactobacilli in stool samples compared with standard infant nutrition $(9,29,30)$. pAOS in infant nutrition did not affect these parameters, but a combination of scGOS/lcFOS and pAOS increased fecal bifidobacteria and lactobacilli as well (12). In the current study, pAOS supplementation did not affect parameters of the fecal microbiota, but scGOS/lcFOS and combinations of scGOS/lcFOS and pAOS did. Despite the differences in gut physiology between mice and man, these results indicate that the prebiotic effects of oligosaccharides in current murine model system are similar to the human situation.

pAOS supplementation alone enhanced DTH responsiveness, but did not affect the proportions of bifidobacteria and lactobacilli in experiment 2, leaving the possibility open that the immune-modulatory effect of dietary pAOS is not causally related to changes in the microbiota. On the other hand, pAOS might have caused changes in certain microbiota parameters that were causally related to the immune-modulatory effects but were not measured. Either way, the observed differences in the effects of scGOS/lcFOS and pAOS on the levels of bifidobacteria and lactobacilli indicate differences in the working mechanisms of the oligosaccharides, which might explain the observed results in the groups receiving combinations of scGOS/lcFOS and pAOS on the DTH responses.

In conclusion, the data presented here show that pAOS supplementation induces Th1skewing effects and stimulatory effects on vaccine-specific DTH responses in a murine model. The data suggest interaction effects between scGOS/lcFOS and pAOS with respect to DTH responses, in parallel to differential effects of the oligosaccharide preparations on the microbiota. Optimal Th1-dependent DTH responses were observed by using combinations of scGOS/lcFOS and pAOS, indicating that this concept holds promise for application in an infant nutrition, with the aim to mimic breast milk as close as possible in composition and function. Clinical trials will need to be performed to determine the efficacy of this concept in infants, for use in situations when breastfeeding is not possible. 


\section{References}

1.

90

2. Coppa, G.V., S. Bruni, L. Morelli, S. Soldi, and O. Gabrielli, The first prebiotics in humans: human milk oligosaccharides. J Clin Gastroenterol, 2004. 38(6 Suppl): p. S80-3.

3. Andersson, B., O. Porras, L.A. Hanson, T. Lagergard, and C. Svanborg-Eden, Inhibition of attachment of Streptococcus pneumoniae and Haemophilus influenzae by human milk and receptor oligosaccharides. J Infect Dis, 1986. 153(2): p. 232-7.

4. Cravioto, A., A. Tello, H. Villafan, J. Ruiz, S. del Vedovo, and J.R. Neeser, Inhibition of localized adhesion of enteropathogenic Escherichia coli to HEp-2 cells by immunoglobulin and oligosaccharide fractions of human colostrum and breast milk. J Infect Dis, 1991. 163(6): p. 1247-55

Newburg, D.S., L.K. Pickering, R.H. McCluer, and T.G. Cleary, Fucosylated oligosaccharides of human milk protect suckling mice from heat-stabile enterotoxin of Escherichia coli. J Infect Dis, 1990. 162(5): p. $1075-80$.

6. Ruiz-Palacios, G.M., J.J. Calva, L.K. Pickering, Y. Lopez-Vidal, P. Volkow, H. Pezzarossi, and M.S. West, Protection of breast-fed infants against Campylobacter diarrhea by antibodies in human milk. J Pediatr, 1990. 116(5): p. 707-13.

7. Eiwegger, T., B. Stahl, J. Schmitt, G. Boehm, M. Gerstmayr, J. Pichler, E. Dehlink, C. Loibichler, R. Urbanek, and Z. Szepfalusi, Human milk--derived oligosaccharides and plant-derived oligosaccharides stimulate cytokine production of cord blood T-cells in vitro. Pediatr Res, 2004. 56(4): p. 536-40.

8. Boehm, G. and B. Stahl, Oligosaccharides, in Functional dairy products, T. Mattila-Sandholm, Editor. 2003, Woodhead: Cambridge. p. 203-43.

9. Boehm, G., S. Fanaro, J. Jelinek, B. Stahl, and A. Marini, Prebiotic concept for infant nutrition. Acta Paediatr Suppl, 2003. 91(441): p. 64-7.

10. Finke, B., B. Stahl, A. Pfenninger, M. Karas, H. Daniel, and G. Sawatzki, Analysis of high-molecularweight oligosaccharides from human milk by liquid chromatography and MALDI-MS. Anal Chem, 1999. 71(17): p. 3755-62.

11. Stahl, B., S. Thurl, J. Zeng, M. Karas, F. Hillenkamp, M. Steup, and G. Sawatzki, Oligosaccharides from human milk as revealed by matrix-assisted laser desorption/ionization mass spectrometry. Anal Biochem, 1994. 223(2): p. 218-26.

12. Fanaro, S., J. Jelinek, B. Stahl, G. Boehm, R. Kock, and V. Vigi, Acidic oligosaccharides from pectin hydrolysate as new component for infant formulae: effect on intestinal flora, stool characteristics, and pH. J Pediatr Gastroenterol Nutr, 2005. 41(2): p. 186-90.

13. Kastner, U., S. Glasl, B. Follrich, J.P. Guggenbichler, and J. Jurenitsch, [Acid oligosaccharides as the active principle of aqueous carrot extracts for prevention and therapy of gastrointestinal infections]. Wien Med Wochenschr, 2002. 152(15-16): p. 379-81.

14. Haarman, M. and J. Knol, Quantitative real-time PCR analysis of fecal Lactobacillus species in infants receiving a prebiotic infant formula. Appl Environ Microbiol, 2006. 72(4): p. 2359-65.

15. Haarman, M. and J. Knol, Quantitative real-time PCR assays to identify and quantify fecal Bifidobacterium species in infants receiving a prebiotic infant formula. Appl Environ Microbiol, 2005. 71(5): p. 2318-24.

16. Nadkarni, M.A., F.E. Martin, N.A. Jacques, and N. Hunter, Determination of bacterial load by realtime PCR using a broad-range (universal) probe and primers set. Microbiology, 2002. 148(Pt 1): p. $257-66$. 
17. Liu, W. and D.A. Saint, A new quantitative method of real time reverse transcription polymerase chain reaction assay based on simulation of polymerase chain reaction kinetics. Anal Biochem, 2002. 302(1): p. 52-9.

18. Siegrist, C.A., Neonatal and early life vaccinology. Vaccine, 2001. 19(25-26): p. 3331-46.

19. Pabst, H.F., Immunomodulation by breast-feeding. Pediatr Infect Dis J, 1997. 16(10): p. 991-5.

20. Pabst, H.F., D.W. Spady, L.M. Pilarski, M.M. Carson, J.A. Beeler, and M.P. Krezolek, Differential modulation of the immune response by breast- or formula-feeding of infants. Acta Paediatr, 1997. 86(12): p. 1291-7.

21. Sinha, A., J. Madden, D. Ross-Degnan, S. Soumerai, and R. Platt, Reduced risk of neonatal respiratory infections among breastfed girls but not boys. Pediatrics, 2003. 112(4): p. e303.

22. Cushing, A.H., J.M. Samet, W.E. Lambert, B.J. Skipper, W.C. Hunt, S.A. Young, and L.C. McLaren, Breastfeeding reduces risk of respiratory illness in infants. Am J Epidemiol, 1998. 147(9): p. 863-70.

23. Blaymore Bier, J.A., T. Oliver, A. Ferguson, and B.R. Vohr, Human milk reduces outpatient upper respiratory symptoms in premature infants during their first year of life. J Perinatol, 2002. 22(5): $p$. 354-9.

24. Gdalevich, M., D. Mimouni, M. David, and M. Mimouni, Breast-feeding and the onset of atopic dermatitis in childhood: a systematic review and meta-analysis of prospective studies. J Am Acad Dermatol, 2001. 45(4): p. 520-7.

25. Gdalevich, M., D. Mimouni, and M. Mimouni, Breast-feeding and the risk of bronchial asthma in childhood: a systematic review with meta-analysis of prospective studies. J Pediatr, 2001. 139(2): p. 261-6.

26. van Odijk, J., I. Kull, M.P. Borres, P. Brandtzaeg, U. Edberg, L.A. Hanson, A. Host, M. Kuitunen, S.F. Olsen, S. Skerfving, J. Sundell, and S. Wille, Breastfeeding and allergic disease: a multidisciplinary review of the literature (1966-2001) on the mode of early feeding in infancy and its impact on later atopic manifestations. Allergy, 2003. 58(9): p. 833-43.

27. Hu-Li, J., E.M. Shevach, J. Mizuguchi, J. Ohara, T. Mosmann, and W.E. Paul, B cell stimulatory factor 1 (interleukin 4) is a potent costimulant for normal resting T lymphocytes. J Exp Med, 1987. 165(1): p. 157-72.

28. Mizubuchi, H., T. Yajima, N. Aoi, T. Tomita, and Y. Yoshikai, Isomalto-oligosaccharides polarize Th1like responses in intestinal and systemic immunity in mice. J Nutr, 2005. 135(12): p. 2857-61.

29. Moro, G.E., F. Mosca, V. Miniello, S. Fanaro, J. Jelinek, B. Stahl, and G. Boehm, Effects of a new mixture of prebiotics on faecal flora and stools in term infants. Acta Paediatr Suppl, 2003. 91(441): p. 77-9.

30. Schmelzle, H., S. Wirth, H. Skopnik, M. Radke, J. Knol, H.M. Bockler, A. Bronstrup, J. Wells, and C. Fusch, Randomized double-blind study of the nutritional efficacy and bifidogenicity of a new infant formula containing partially hydrolyzed protein, a high beta-palmitic acid level, and nondigestible oligosaccharides. J Pediatr Gastroenterol Nutr, 2003. 36(3): p. 343-51. 


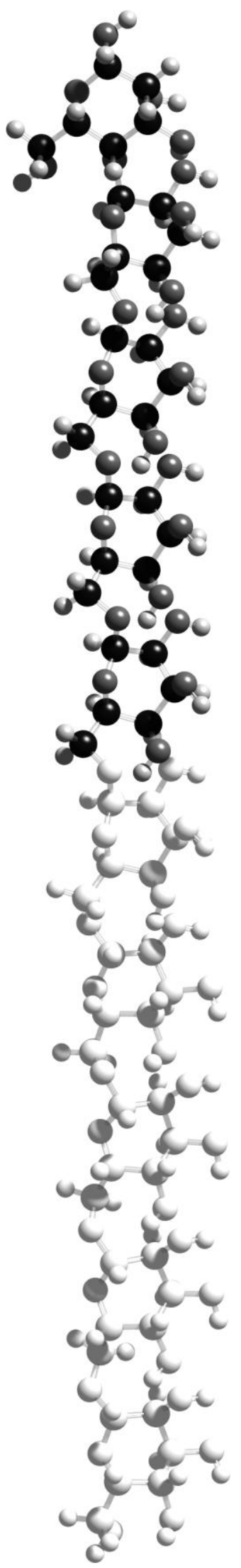

Effects of a specific mixture of oligosaccharides in a murine vaccination model: supplementation around the priming phase enhances cellular immune responses that are correlated with cecal lactobacilli

$$
\begin{array}{r}
\text { A.P. Vos } \\
\text { M. Haarman } \\
\text { J. Knol } \\
\text { B. Stahl } \\
\text { L. M'Rabet } \\
\text { J. Garssen }
\end{array}
$$




\section{Abstract}

It is commonly hypothesized that immune modulatory effects of prebiotic agents are caused by changes in the composition of the intestinal microbiota, where prebiotics stimulate the growth of beneficial bacteria such as bifidobacteria and lactobacilli. Under this hypothesis, a correlation between these bacteria and the immune modulatory effects would be expected. This was investigated using dietary supplementation with a specific mixture of short-chain galactooligosaccharides and long-chain fructooligosaccharides in a 9:1 ratio (Immunofortis), in combination with pectinderived acidic oligosaccharides, in a murine vaccination model. An experiment was included in which the timing of supplementation was varied relative to the vaccination scheme, to find the most relevant time point to analyze the potential correlation.

By varying the timing of prebiotic supplementation around two vaccinations at day 0 and 21, it was shown that supplementation was necessary before the first vaccination, but not after day 8, to increase delayed-type hypersensitivity responses (DTH) significantly at day 30. This suggested that the immune modulatory effect occurred during the priming phase of the response. Therefore, the correlation between microbiota-related parameters and DTH responses was analyzed shortly after the primary vaccination, using a shortened vaccination protocol. Multiple regression analysis showed that the percentages of lactobacilli in the cecum were significantly correlated to the DTH responses, although the correlation was not strong. Furthermore, the results of the analysis suggested that other factors played a role as well. Therefore, additional research is necessary to unravel further details of the immune modulatory mechanisms of prebiotic oligosaccharides.

\section{Introduction}

It was recently shown that specific mixtures of prebiotic oligosaccharides induce immune-modulatory effects in several murine models. Enhanced T-helper 1 (Th1)related delayed-type hypersensitivity (DTH) responses were observed repeatedly in a C57BL/6 influenza vaccination model (chapters 3 and 4). In addition, decreased parameters of airway hyperresponsiveness and inflammation were observed in a model of experimentally induced allergic asthma in Balb/c mice (chapter 8). The oligosaccharide mixtures that were used consisted of short-chain galacto- 
oligosaccharides (scGOS) and long-chain fructooligosaccharides (lcFOS) in a 9:1 ratio (Immunofortis), alone or in combination with partly unsaturated and methylated pectin-derived acidic oligosaccharides (pAOS).

Prebiotic effects of these oligosaccharide mixtures were shown in human infants. The proportions of bifidobacteria and lactobacilli were increased in infants that received a supplemented formula, compared to a control formula. As a result, the composition of the microbiota in the oligosaccharide-fed infants was more similar to the bifidobacteriadominated microbiota in breast-fed infants (1-3). We have observed similar effects of these mixtures on the microbiota in murine models. Therefore, a likely cause of the observed immune modulation was a change in the interaction between the microbiota and the immune system, caused by the prebiotic intervention. However, when several different oligosaccharides were tested in a murine vaccination model, some oligosaccharide preparations induced prebiotic effects without measurable effects on the DTH responses and vice versa (chapters 3 and 4 ).

These results raised questions about the mechanisms underlying the immunemodulatory effects and the role of the microbiota. Under the common assumption that oligosaccharide-induced immune modulation is mediated via changes in the microbiota, a correlation is expected to occur between microbiota and immunological parameters. The aim of the present study was to investigate this potential correlation, in order to find evidence for the role of the microbiota in oligosaccharide-induced immune modulation. In a first experiment, the timing of oligosaccharide supplementation was varied in a vaccination experiment, to identify the phase of the immune response at which the modulatory effect occurred. Because the oligosaccharides apparently modulated the immune response during the priming phase, the correlation analysis was performed in a shortened vaccination experiment.

\section{Materials and methods}

\section{Mice}

Female, 6 week-old C57Bl/6JOlaHsd mice were obtained from Harlan (Horst, The Netherlands) and kept under normal conditions, with a $12 \mathrm{~h}$ dark and light cycle and access to food and water ad libitum. The animals received control AIN-93G diets and routine care for a minimum of 5 days upon arrival in the animal facility, before the start of the experiments. All experiments were approved by an independent animal experiments committee (DEC Consult, Bilthoven, The Netherlands). 


\section{Diets}

All animals received semi-purified AIN-93G-based diets (Research Diet Services, Wijk bij Duurstede, The Netherlands). The supplemented and control diets were described previously (chapter 4). In short, $0.9 \%$ (w/w) net scGOS/lcFOS in a 9:1 ratio (Immunofortis)and $0.15 \%(\mathrm{w} / \mathrm{w})$ net pAOS were added to the test diet. The amount of added carbohydrates in the test diet was exchanged for the same amount of total carbohydrates in the control diet. The oligosaccharides were mixed into the AIN-93G diet and pressed into pellets. pAOS were derived from pectin (kindly donated by Südzucker AG, Mannheim, Germany). scGOS/lcFOS was used as a spray-dried powder of scGOS (Vivinal GOS, Borculo Domo, Zwolle, The Netherlands) and 1cFOS with a high degree of polymerization (Raftiline HP, Orafti, Wijchen, The Netherlands) in a 9:1 ratio. Maltodextrin, glucose and lactose were present as carrier materials in the scGOS/lcFOS powder. These carbohydrates were also exchanged for total carbohydrates in the control diet and were previously shown not to affect vaccination responses (chapter 3).

\section{Vaccination protocol and DTH response}

The vaccination protocol that was used in this study has been described previously (chapter 3). This protocol was shown to induce sub-maximal immune responses that allow the detection of stimulatory as well as inhibitory immune-modulatory effects. In a first experiment, the mice received primary and booster vaccinations, consisting of a subcutaneous (sc) injection of a 1:1 mix of vaccine solution (100x diluted Influvac 2002/2003 in PBS, Solvay Pharmaceuticals, Weesp, The Netherlands) and adjuvant (Stimune, Cedi-diagnostics, Lelystad, The Netherlands). Sham vaccination groups received injections of a 1:1 mix of PBS and adjuvant, to show the specificity of the vaccination response $(n=3$; all other groups $n=10)$. Primary and booster vaccinations were given at days 0 and 21. Delayed-type hypersensitivity responses (DTH) reactions were induced at day 29 by sc injection of $25 \mu \mathrm{L}$ Influvac into the ear pinnae of both ears. Ear thickness was measured in duplicate before antigen challenge, as well as 24 hours afterwards (day 30), with a digital micrometer (Mitutoyo Digimatic 293561, Veenendaal, The Netherlands). The DTH response was calculated by subtracting the basal ear thickness from the value at 24 hours after challenge.

In a second experiment, the booster vaccination was omitted to reduce the length of the protocol: the DTH response was initiated 8 days after the primary vaccination and was measured at day 9. In addition, two antigen concentrations were used (10x and 100x diluted Influvac 2002/2003 in PBS) to test this shortened vaccination scheme and make 
sure that the DTH responses were detectible at day 9.

\section{Dietary intervention}

The timing of the dietary intervention was varied in the first experiment. The control group received the control diet from day -14 to 30 . Five supplementation groups received the supplemented diet for different lengths of time during the vaccination protocol: day -14 to 30 (A), day 0 to 30 (B), day 21 to 30 (C), day 29 to 30 (D) and day -14 to 8 (E; no supplementation from day 9 to 30 ) (see also Fig. 1a). This experiment was performed in two cohorts. In the first cohort, sham and control groups were compared with groups A, B, C and D. In the second cohort, sham and control groups were compared with groups $\mathrm{A}$ and $\mathrm{E}$. The sham group, control group and group A were measured in both cohorts, leading to a pooled sham group of $n=6$, control group of $\mathrm{n}=20$ and group $\mathrm{A}$ of $\mathrm{n}=20$ animals. All other groups consisted of $\mathrm{n}=10$ animals.

Dietary intervention with the supplemented diet lasted from day -14 to 9 in the second experiment. Separate control and test groups were included for the two antigen concentrations that were tested.

\section{Quantitative real-time PCR of the genus Bifidobacterium and Lactobacillus}

For the relative quantification of the genera Bifidobacterium and Lactobacillus in relation to the total bacterial load, a duplex 5' nuclease quantitative real-time PCR (qPCR) assay was used as previously published $(4,5)$. The relative amounts of the genus Bifidobacterium or Lactobacillus in the samples were calculated with respect to the total bacterial load according to Liu et al. (6), and expressed in percentages. All samples were measured in triplicate.

\section{Statistics}

Statistical calculations were performed in SPSS version 15 (SPSS Inc, Chicago, IL, USA), graph plotting was performed in SPSS and Graphpad Prism 4.03 software (Graphpad Software Inc, San Diego, CA, USA). Differences between one control group and multiple test groups were analyzed using ANOVA with post-hoc Dunnett's test. More elaborate comparisons between groups were analyzed using ANOVA with post-hoc Bonferroni's test. The correlation between parameters of the microbiota and DTH values were analyzed by multiple regression analysis. The significance of adding parameters to the regression model was evaluated by the resulting change in the Fstatistic. In the first experiment, the DTH data from both cohorts were calculated 
relative to the mean of the control groups and were pooled subsequently. The same analyses were performed as well for both cohorts separately using the unpooled data, yielding the same statistical conclusions (data not shown). All microbiota parameters were transformed logarithmically before statistical analysis, in order to normalize the data distribution and linearize the relation with DTH responses. For all analyses, pvalues $<0.05$ were considered to be statistically significant. Data is represented in this paper as mean $\pm \mathrm{SEM}$; error bars in graphs represent SEM.

\section{Results}

Similar to previous experiments, continuous supplementation from day -14 to 30 with the test diet (group A) increased vaccine-specific DTH responses significantly compared to control mice $(157.8 \pm 6.0 \%$ vs. $100.0 \pm 4.1 \%$; $<0.001$; Figure $1 b)$. In contrast, no significant effect was observed when supplementation started at later time points (groups B-D), although group B showed a trend (123.2 $\pm 6.7 \%$; $=0.06)$. However, when the supplementation diet was given from day -14 to 8 (group E), the DTH response at day 30 was similar to group A $(151.6 \pm 9.1 ; \mathrm{p}<0.001$ compared to controls). No increase in ear thickness was observed in the sham group.

The percentages of bifidobacteria and lactobacilli were measured in colon samples in individual mice at the end of the first experiment (Figure 1c-e). The intra-group variation was high for both parameters. Overall, a trend of higher percentages of bifidobacteria and lactobacilli with longer supplementation was observed. The percentage of lactobacilli was significantly increased in groups A and C compared to the control group $(5.6 \pm 1.1 \%$ and $4.8 \pm 1.0 \%$ vs. $1.3 \pm 0.4 \%$; $=0.003$ and $p=0.004$, respectively). Group B showed lower percentages of lactobacilli than groups A and C, but a higher percentage of bifidobacteria. When the percentages of bifidobacteria and lactobacilli were added, this resulted in a gradual effect of the length of supplementation on the total percentage of both bacterial genera; groups A and B both had significantly higher percentages than the control mice $(14.7 \pm 3.1$ and $13.3 \pm 4.3 \mathrm{vs}$. $4.1 \pm 1.2 ; \mathrm{p}=0.003$ and $\mathrm{p}=0.02$, respectively).

The results from the first experiment indicated that the prebiotic effects on the microbiota at the end of the experiment were not necessarily linked to the immune stimulatory effect on the DTH response, particularly in group E. Because the results suggested that the immune-modulatory effect of the specific oligosaccharide mixture occurred around the priming phase of the immune response, it was decided to shorten 

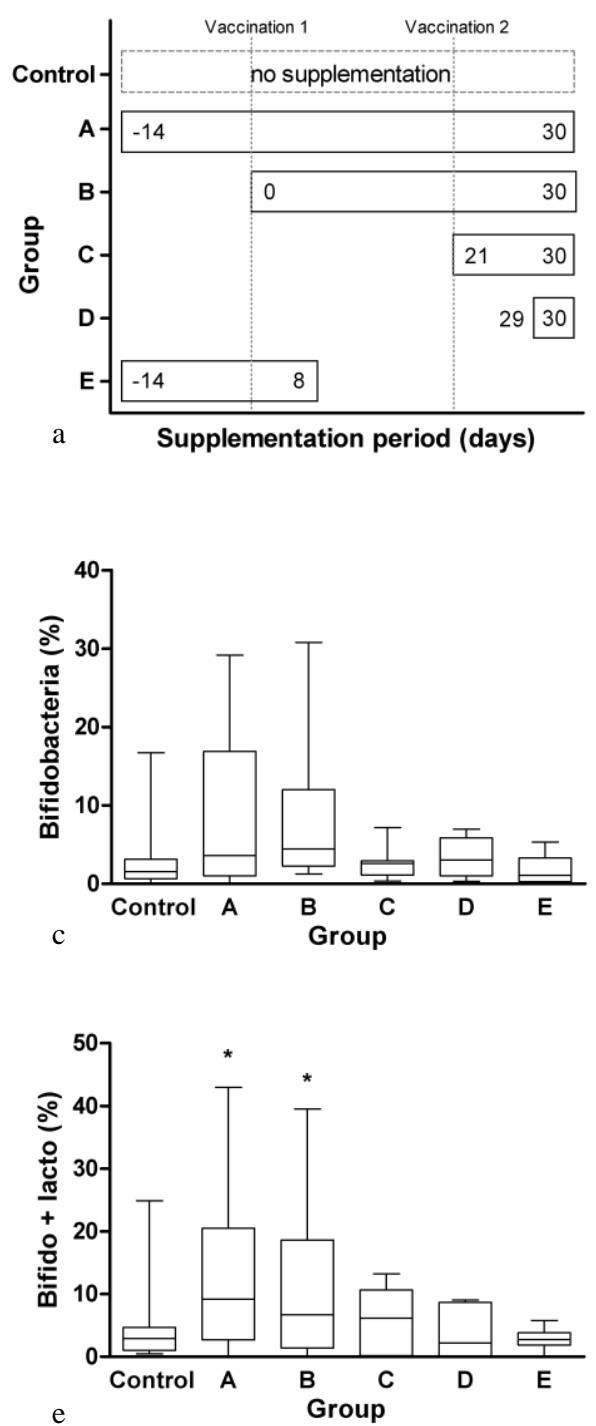
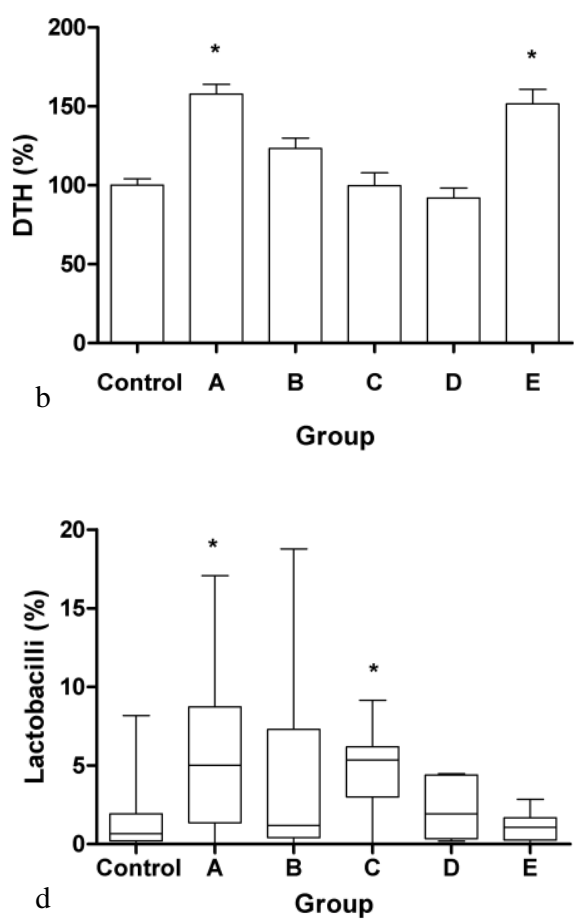

Figure 1. Effects of variation in the timing of dietary supplementation on vaccine-specific DTH responses and microbiota parameters. (a) The timing of the dietary intervention in the different experimental groups is schematically depicted. Primary and booster vaccinations were administered at days o and 21, respectively. (b) Vaccine-specific DTH responses were measured at day 30. The experiment was performed in two cohorts, therefore the DTH results are shown relative to the control group. Bars represent group averages \pm SEM. The percentages of bifidobacteria (c) and lactobacilli (d) were measured in colon samples, relative to the number of total bacteria. The total percentage of 'beneficial bacteria' is shown as the sum of the percentages of bifidobacteria and lactobacilli (e). Box-and-whisker plots show the data distribution (median, interquartile range and extremes); microbiota-related data were transformed logarithmically before analysis to normalize the distribution. * Indicates $p<0.05$ in all graphs. 
the vaccination protocol, in order to study the prebiotic effects closer in time to the priming phase. Using the same dietary supplementation as in the first experiment and starting 14 days before the primary vaccination, the DTH responses were increased significantly compared to control mice when measured at day 9 after primary vaccination in the second experiment (Figure 2a). Two antigen doses were used: the high dose resulted in a significant increase in DTH response compared to the low dose $(141.0 \pm 7.3 \mu \mathrm{m}$ vs. $107.9 \pm 8.2 \mu \mathrm{m} ; \mathrm{p}=0.01)$. For both antigen doses, supplementation further increased DTH responses significantly. No increase in ear thickness was observed in the sham group.
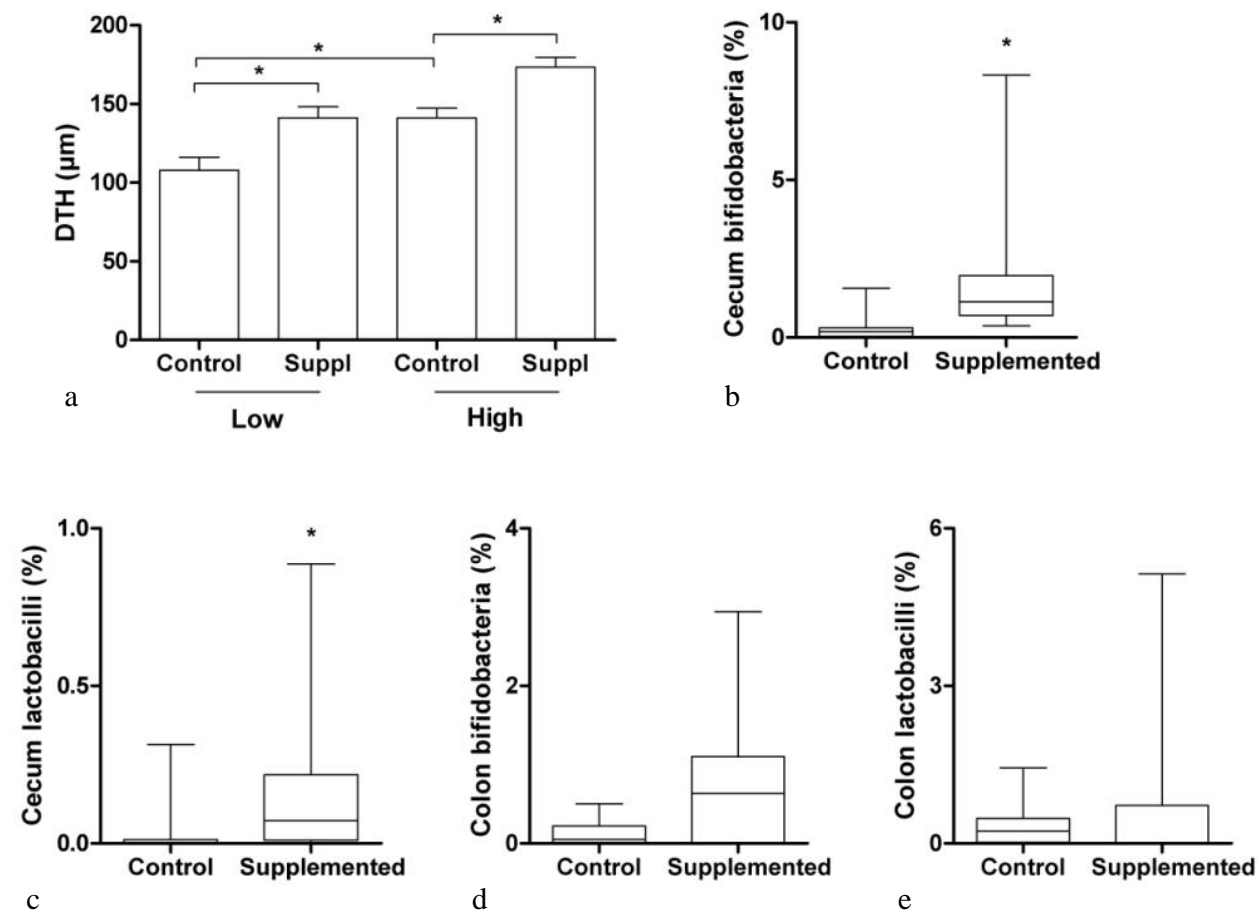

Figure 2. Dietary supplementation enhances DTH responses in a shortened vaccination model and induce prebiotic effects in the cecum. A shortened vaccination model was used to study the relationship between the immune modulatory and prebiotic effects. A single vaccination was administered at day o, dietary intervention lasted from day -14 to 9 . DTH responses and microbiota-related parameters were measured at day 9. (a) Supplementation with the specific mixture of oligosaccharides significantly enhanced DTH responses, both at a low (100x diluted vaccine) and high (10x diluted vaccine) antigen concentration. Bars represent group averages \pm SEM. The percentages of bifidobacteria and lactobacilli were measured in cecum $(b, c)$ and colon $(d, e)$ samples, relative to the number of total bacteria. Box-and-whisker plots show the data distribution (median, interquartile range and extremes); microbiota-related data were transformed logarithmically before analysis to normalize the distribution. * Indicates $p<0.05$ compared to the control animals, or to other groups as indicated by horizontal lines. 
Due to the low amount of material isolated from the cecum and colon of the animals, 37 samples of cecal content and only 28 samples of colonic content were successfully analyzed for the percentages of bifidobacteria and lactobacilli (Figure 2b-e). The percentages of both groups of bacteria were significantly increased in the cecum samples of supplemented mice.

The relationship between the microbiota parameters and DTH response was analyzed by means of multiple regression. The regression model always contained the antigen dose variable, to take into account the two antigen doses that were used. The significance of adding single microbiota-related parameters into the model was tested, as well as combinations of these parameters (Table 1). Inclusion of the cecal microbiota parameters significantly improved the model, but only the percentage of lactobacilli showed a significant positive correlation with the DTH data. When the diet group data (supplemented yes/no) was used instead of the cecal lactobacilli, a similar model fit was observed. In a combined model, the antigen dose, diet group and cecal lactobacilli variables all contributed significantly ( $\mathrm{p}<0.001, \mathrm{p}=0.01$ and $\mathrm{p}=0.03$, respectively), leading to an optimal model fit $\left(\mathrm{R}^{2}=0.63\right)$. The partial correlation plot of the cecal lactobacilli and DTH response is shown in Figure 3, depicting the relationship between both parameters while all other factors are kept constant.

In the unsupplemented group, many samples had percentages of lactobacilli in the cecum that were below the detection limit of the assay, however, exclusion of these data points did not change the outcome of the analysis.

Inclusion of either or both of the colonic microbiota parameters into the model did not improve it. However, when the colonic bifidobacteria were analyzed excluding the samples below the detection limit, a significant correlation with DTH responses was observed (data not shown). The difference in these statistical outcomes was caused by a relatively high number of animals in the supplemented group that had undetectable levels of colonic bifidobacteria (5 out of 15 observations). Therefore, excluding these samples from the analysis introduces an unwanted bias.

\section{Discussion}

It is demonstrated in this study that dietary supplementation with a specific mixture of scGOS, lcFOS and pAOS increased vaccine-specific DTH responses in C57BL/6 mice, as reported previously (chapter 4). In a first step, it was shown that the immunemodulatory effect occurred during the priming phase of the response. Indeed, supplementation from day -14 to 8 yielded a similar increase in DTH response as 
Table 1. Multiple regression analysis of the relationship between the DTH responses and microbiota-related parameters.

\begin{tabular}{|c|c|c|c|c|c|c|}
\hline Regression model & Independent variables & $\mathrm{R}^{2}$ & Adjusted $\mathrm{R}^{2}$ & $\begin{array}{l}\text { Degrees of } \\
\text { freedom }\end{array}$ & $\begin{array}{l}\text { Significance of } \\
\text { F change }^{a}\end{array}$ & $\begin{array}{l}\text { Significance of } \\
\text { coefficient }^{b}\end{array}$ \\
\hline \multirow{2}{*}{$\begin{array}{l}1 \text { No microbiota } \\
\text { parameters }\end{array}$} & Antigen concentration & 0.262 & 0.241 & 35 & 0.001 & $<0.001$ \\
\hline & Supplementation group & 0.568 & 0.543 & 34 & $<0.001$ & $<0.001$ \\
\hline \multirow{4}{*}{$\begin{array}{l}2 \text { Cecum } \\
\text { parameters }\end{array}$} & Antigen concentration & 0.262 & 0.241 & 35 & 0.001 & $<0.001$ \\
\hline & Cecum bifidobacteria & 051 & ב & 2 & $<0$ & 0.143 \\
\hline & Cecum lactobacilli & 0.344 & 0.505 & 33 & rest & 0.010 \\
\hline & Supplementation group & 0.651 & 0.607 & 32 & 0.004 & 0.004 \\
\hline \multirow{3}{*}{$\begin{array}{l}3 \text { Cecum } \\
\text { Lactobacilli }\end{array}$} & Antigen concentration & 0.262 & 0.241 & 35 & 0.001 & $<0.001$ \\
\hline & Cecum lactobacilli & 0.543 & 0.516 & 34 & $<0.001$ & 0.030 \\
\hline & Supplementation group & 0.626 & 0.592 & 33 & 0.010 & 0.010 \\
\hline \multirow{4}{*}{$\begin{array}{l}4 \text { Colon } \\
\text { parameters }\end{array}$} & Antigen concentration & 0.379 & 0.355 & 26 & $<0.001$ & $<0.001$ \\
\hline & Colon bifidobacteria & 0 & 0.2 & $2 /$ & & 0.667 \\
\hline & Colon lactobacilli & & 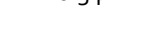 & & - & 0.501 \\
\hline & Supplementation group & 0.640 & 0.578 & 23 & 0.001 & 0.001 \\
\hline
\end{tabular}

${ }^{a}$ Each of the four regression models was analyzed by adding (groups of) parameters in a step-wise manner, ordered from top to bottom and indicated by indentation of the variables. The significance of the change in $\mathrm{R} 2$ after each step is shown, as calculated by the change in the $F$ statistic.

${ }^{\mathrm{b}} \mathrm{T}$ he significance of the regression coefficients was calculated after all variables were entered into the model.

observed in continuously supplemented mice, while supplementation from day 0 to 30 did not result in a significant increase, although the total supplementation period was longer in the latter group. These findings indicate that it takes some time for the modulatory effect to occur, which might be related to the time it takes for the microbiota to adapt following the supplementation.

Starting oligosaccharide supplementation on the day of primary vaccination (group B) increased the DTH, but the effect was not significant. This was most likely caused by the fact that the priming phase of the immune response lasts for some time after the primary vaccination, causing potential overlap with a gradually developing immune modulatory effect.

The immune modulating effects that were observed in previous experiments - a 


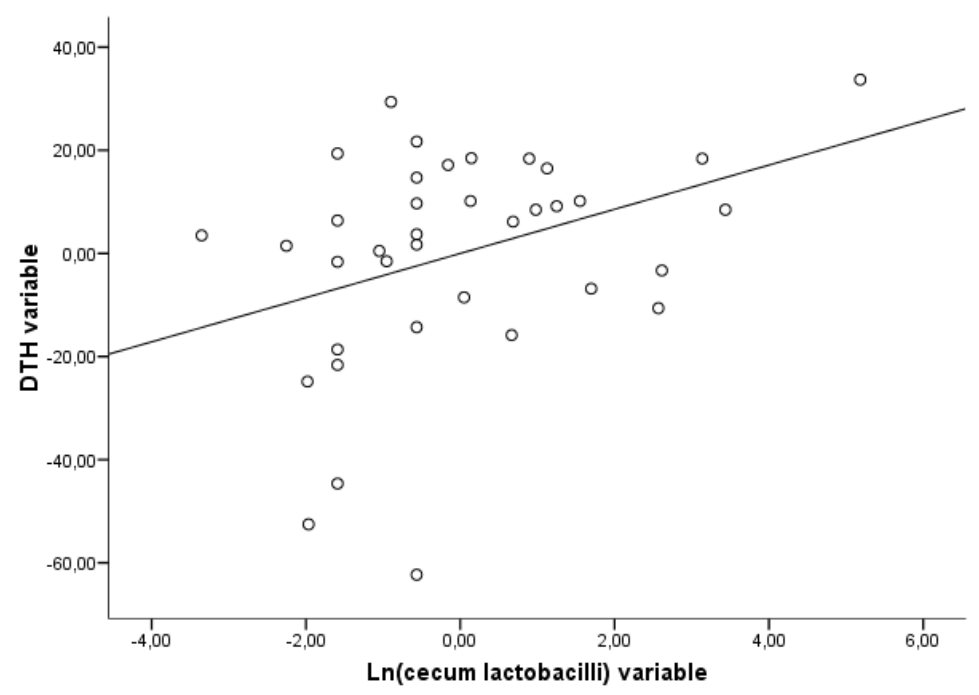

Figure 3. Partial correlation plot of the cecal lactobacilli and the DTH response residuals. Multiple regression analysis was used to analyze the relationship between the microbiota-related parameters and the observed DTH responses in the second experiment. A significant correlation was found between the cecal lactoballi and the DTH responses in a model that included the antigen concentration and supplementation group data as well. The relationship between the cecal lactobacilli and DTH response was visualized by plotting the residuals of both parameters against each other, which have been obtained after regression of both parameters against the other independent variables.

stimulation of Th1 related DTH responses and an inhibition of various parameters of allergic asthma and antigen-specific Th2 cytokine production in splenocyte cultures (chapters 3, 4 and 8) - led us to hypothesize that the immune-modulating effects of the specific oligosaccharide mixtures were due to a shift in the balance between Th1 and Th2 responses. The current results, which suggest that immune modulation occurs during the priming phase, fit well with this hypothesis. The polarization of naïve Tcells into Th1 or Th2 effector cells has been described to occur early in the immune response $(7,8)$. The cytokines IL-4 and IL-12 have been described to be the major determinants in the T-cell polarization process, while various other cytokines, interactions with costimulatory molecules on antigen-presenting cells (APC) and other factors are known to play a role in this process as well (reviewed in 9, 10). It is unknown whether the supposed modulation of T-cell polarization is caused by an effect of supplementation on the T-cells, on APC such as dendritic cells and their interaction with T-cells, or on other cells that cause a form of bystander effect on the vaccination response.

Because the vaccinations were administered subcutaneous at the back of the neck of the 
animals, T-cell priming is expected to have occurred in the axillary lymph nodes (enlargement of these lymph nodes has been observed frequently; data not shown), distant from the gut and its associated mucosal immune tissues. Supplementation with prebiotics has been reported to lead to modulation of mucosal immune parameters, which is suggested to be caused by direct interactions between prebiotics or the bacteria that ferment them and gut-associated lymphoid tissues (11-13). The precise mechanism that is responsible for systemic immune-modulatory effects, induced by dietary oligosaccharides, is currently unclear.

The correlation analyses showed that the percentages of bifidobacteria and lactobacilli were correlated to a similar extent with the DTH response as the diet group data (supplemented yes/no). At first glance, these results do not provide a strong indication for a critical role of bifidobacteria and lactobacilli in the immune-modulatory effect. However, the correlation between the cecal lactobacilli and the DTH response remained significant after addition of the supplementation group variable to the model, indicating that the percentages of cecal lactobacilli explain a significant part of the variation in the DTH response, independent from oligosaccharide supplementation.

The correlation between the cecal lactobacilli and the DTH response is consistent with the hypothesis that immune-modulatory effects of prebiotic oligosaccharides are mediated via the microbiota. However, since the diet group variable always contributed significantly to the regression models, it is most likely that other factors than the ones that were measured played a role as well.

This study focused only on the percentages of bifidobacteria and lactobacilli, which are the most studied groups of bacteria related to prebiotic or probiotic effects. However, other groups or species of bacteria might have played a role as well. In addition, the anatomical location of the bacteria might be important for their effect on the immune system. It has been described that mucosa-associated microbiota are in close contact with the epithelium and mucosal immune cells (reviewed in 14, 15). The microbiota in this layer have been suggested to be of main importance in the interaction with the immune system, and it has been reported that prebiotic supplementation affected the composition of this layer of microbiota $(16,17)$. It is possible that effects of prebiotics are different on luminal microbiota, which were measured in this study, than on mucosa-associated microbiota. This may provide an explanation for the weak correlations that were found. Alternatively, the supplemented oligosaccharides may already modulate the microbiota that are present in the small intestine, thereby modulating the interaction between the local microbiota and Peyer's patch cells and/or other gut-associated lymphoid tissues $(12,13)$. Lastly, direct immune modulatory 
effects of the oligosaccharides might have played a role, as there are indications that small proportions of oligosaccharides can reach the circulation $(18,19)$ and modulate immune responses in a microbiota-independent fashion (20).

Immune modulatory effects of specific mixtures of scGOS and lcFOS, with or without pAOS, have been shown repeatedly in animal models and clinical trials (21, 22; chapters 3, 4, 7 and 8). The current results suggest that oligosaccharide-induced modulation of $\mathrm{T}$ cell-dependent responses occurs at the priming phase of the response. Beneficial effects of prebiotic oligosaccharides are commonly ascribed to their stimulatory effect on the number of intestinal bifidobacteria and/or lactobacilli. Correspondingly, a significant correlation was found in mice between the percentage of cecal lactobacilli and vaccination-specific DTH response in the current study. However, the results of the statistical analysis suggested that other factors may play an important role in the observed immune modulatory effect as well. Therefore, more research is recommended in order to gain a more detailed insight into the underlying mechanism of oligosaccharide-induced immune-modulatory effects, as well as into the similarities and discrepancies between these mechanisms in mice and man.

\section{References}

1. Fanaro, S., J. Jelinek, B. Stahl, G. Boehm, R. Kock, and V. Vigi, Acidic oligosaccharides from pectin hydrolysate as new component for infant formulae: effect on intestinal flora, stool characteristics, and pH. J Pediatr Gastroenterol Nutr, 2005. 41(2): p. 186-9o.

2. Knol, J., P. Scholtens, C. Kafka, J. Steenbakkers, S. Gro, K. Helm, M. Klarczyk, H. Schopfer, H.M. Bockler, and J. Wells, Colon microflora in infants fed formula with galacto- and fructooligosaccharides: more like breast-fed infants. J Pediatr Gastroenterol Nutr, 2005. 40(1): p. 36-42.

3. Moro, G., I. Minoli, M. Mosca, S. Fanaro, J. Jelinek, B. Stahl, and G. Boehm, Dosage-related bifidogenic effects of galacto- and fructooligosaccharides in formula-fed term infants. J Pediatr Gastroenterol Nutr, 2002. 34(3): p. 291-5.

4. Haarman, M. and J. Knol, Quantitative real-time PCR assays to identify and quantify fecal Bifidobacterium species in infants receiving a prebiotic infant formula. Appl Environ Microbiol, 2005. 71(5): p. 2318-24.

5. Haarman, M. and J. Knol, Quantitative real-time PCR analysis of fecal Lactobacillus species in infants receiving a prebiotic infant formula. Appl Environ Microbiol, 2006. 72(4): p. 2359-65.

6. Liv, W. and D.A. Saint, A new quantitative method of real time reverse transcription polymerase chain reaction assay based on simulation of polymerase chain reaction kinetics. Anal Biochem, 2002. 302(1): p. 52-9.

7. Hsieh, C.S., A.B. Heimberger, J.S. Gold, A. O'Garra, and K.M. Murphy, Differential regulation of T helper phenotype development by interleukins 4 and 10 in an alpha beta T-cell-receptor transgenic system. Proc Natl Acad Sci U S A, 1992. 89(13): p. 6065-9. 
8. Seder, R.A., W.E. Paul, M.M. Davis, and B. Fazekas de St Groth, The presence of interleukin 4 during in vitro priming determines the lymphokine-producing potential of $\mathrm{CD}_{4}+\mathrm{T}$ cells from $\mathrm{T}$ cell receptor transgenic mice. J Exp Med, 1992. 176(4): p. 1091-8.

9. O'Garra, A., Cytokines induce the development of functionally heterogeneous T helper cell subsets. Immunity, 1998. 8(3): p. 275-83.

10. Murphy, K.M. and S.L. Reiner, The lineage decisions of helper T cells. Nat Rev Immunol, 2002. 2(12): p. $933-44$.

11. Forchielli, M.L. and W.A. Walker, The role of gut-associated lymphoid tissues and mucosal defence. Br J Nutr, 2005. 93 Suppl 1: p. S41-8.

12. Hosono, A., A. Ozawa, R. Kato, Y. Ohnishi, Y. Nakanishi, T. Kimura, and R. Nakamura, Dietary fructooligosaccharides induce immunoregulation of intestinal IgA secretion by murine Peyer's patch cells. Biosci Biotechnol Biochem, 2003. 67(4): p. 758-64.

13. Roller, M., A. Pietro Femia, G. Caderni, G. Rechkemmer, and B. Watzl, Intestinal immunity of rats with colon cancer is modulated by oligofructose-enriched inulin combined with Lactobacillus rhamnosus and Bifidobacterium lactis. Br J Nutr, 2004. 92(6): p. 931-8.

14. Everett, M.L., D. Palestrant, S.E. Miller, R.R. Bollinger, and W. Parker, Immune exclusion and immune inclusion: A new model of host-bacterial interactions in the gut. Clin Appl Immunol Rev, 2004. 4: p. 321-332.

15. Bollinger, R.R., M.L. Everett, D. Palestrant, S.D. Love, S.S. Lin, and W. Parker, Human secretory immunoglobulin A may contribute to biofilm formation in the gut. Immunology, 2003. 109(4): p. 5807.

16. Kleessen, B. and M. Blaut, Modulation of gut mucosal biofilms. Br J Nutr, 2005. 93 Suppl 1: p. S35-40.

17. Langlands, S.J., M.J. Hopkins, N. Coleman, and J.H. Cummings, Prebiotic carbohydrates modify the mucosa associated microflora of the human large bowel. Gut, 2004. 53(11): p. 1610-6.

18. Molis, C., B. Flourie, F. Ouarne, M.F. Gailing, S. Lartigue, A. Guibert, F. Bornet, and J.P. Galmiche, Digestion, excretion, and energy value of fructooligosaccharides in healthy humans. Am J Clin Nutr, 1996. 64(3): p. 324-8.

19. Obermeier, S., S. Rudloff, G. Pohlentz, M.J. Lentze, and C. Kunz, Secretion of 13 C-labelled oligosaccharides into human milk and infant's urine after an oral [13C]galactose load. Isotopes Environ Health Stud, 1999. 35(1-2): p. 119-25.

20. Sonoyama, K., H. Watanabe, J. Watanabe, N. Yamaguchi, A. Yamashita, H. Hashimoto, E. Kishino, K. Fujita, M. Okada, S. Mori, S. Kitahata, and J. Kawabata, Allergic airway eosinophilia is suppressed in ovalbumin-sensitized Brown Norway rats fed raffinose and alpha-linked galactooligosaccharide. J Nutr, 2005. 135(3): p. 538-43.

21. Moro, G., S. Arslanoglu, B. Stahl, J. Jelinek, U. Wahn, and G. Boehm, A mixture of prebiotic oligosaccharides reduces the incidence of atopic dermatitis during the first six months of age. Arch Dis Child, 2006. 91(10): p. 814-9.

22. Arslanoglu, S., G. Moro, J. Schmitt, and G. Boehm, Early dietary intervention with a mixture of prebiotic oligosaccharides reduces the incidence of allergy associated symptoms and infections during the first 2 years of life. J Pediatr Gastroenterol Nutr, 2007. 44(S1): p. e217. 


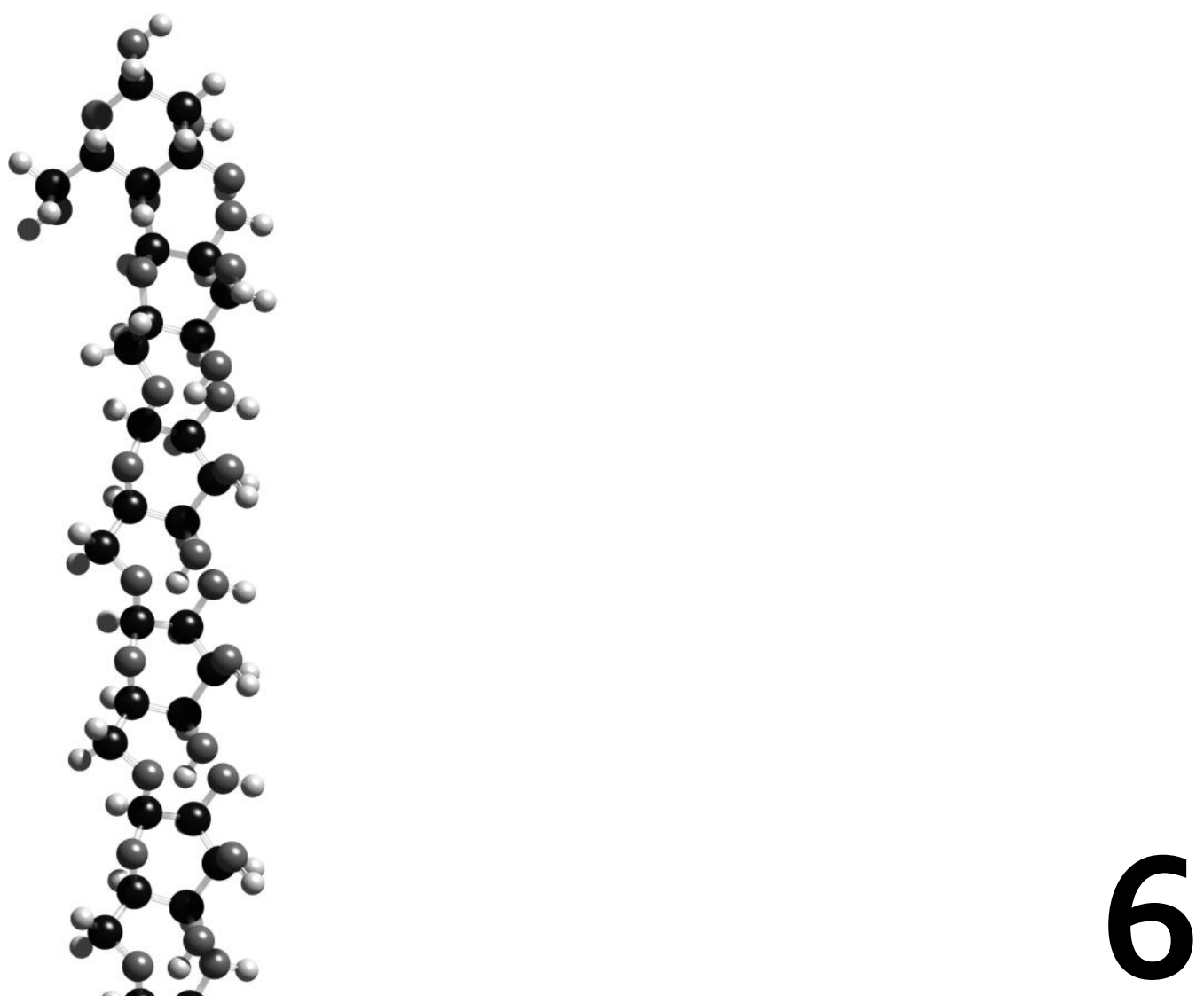

No evidence for immune-modulatory effects of specific neutral and acidic oligosaccharides in a limited set of in vitro assays

A.P. Vos J.W.H. van Ginkel

B. Sprangers

L. M'Rabet

J. Garssen 


\section{Abstract}

A specific mixture of short-chain galactooligosaccharides and long-chain fructooligosaccharides in a 9:1 ratio (Immunofortis), especially in combination with pectin-derived acidic oligosaccharides (pAOS), appeared to modulate $\mathrm{T}$ cell polarization and natural killer (NK) activity in previous animal experiments. It was investigated whether immune modulatory effects of these specific oligosaccharides were detectable in vitro.

Dendritic cell (DC) maturation and T cell function was studied, because both cell types play important roles in the induction and polarization of $\mathrm{T}$ cell responses. $\mathrm{T}$ cell proliferation and cytokine production was analyzed in Concanavalin A-stimulated peripheral blood mononuclear cells (PBMC). DC maturation was studied in human immature monocyte-derived DCs, by analyzing the expression of maturation and costimulatory markers. In addition, the effect of the specific oligosaccharides was studied on NK activity in PBMC. A wide range of oligosaccharide concentrations were tested, widely spanning biologically relevant concentrations. However, addition of the oligosaccharides to the culture medium did not lead to consistent effects in any of the in vitro assays.

In conclusion, no evidence was found for immune-modulatory effects of specific oligosaccharides in a limited set of in vitro assays. Additional in vitro experiments are recommended to study potentially relevant immunological mechanisms.

\section{Introduction}

Specific mixtures of oligosaccharides have been previously shown to induce immune modulatory effects in vivo (chapters 3-5). A mixture of short-chain galactooligosaccharides (scGOS) and long-chain fructooligosaccharides (lcFOS) in a 9:1 ratio (Immunofortis), especially in combination with pectin-derived acidic oligosaccharides (pAOS), repeatedly enhanced vaccine-specific delayed-type hypersensitivity (DTH) responses in a murine influenza vaccination model. In addition, a reduction in ex vivo T-helper 2 (Th2) cytokine production was observed (chapter 4), as well as reduced parameters of allergic asthma (chapter 8). Overall, these results strongly suggest that the oligosaccharides induce a shift in the Th1/Th2 balance towards Th1 responses. 
There are also indications from animal experiments that these specific oligosaccharides enhance natural killer (NK) activity. In an experiment with 8 months old senescenceaccelerated SAMP8 mice, supplementation with up to $5 \%$ of scGOS/lcFOS/pAOS led to a significant and dose-dependent increase in splenocyte NK activity (unpublished data). In addition, an inhibitory effect of oligosaccharide supplementation was observed on viral load in the early phase of cytomegalovirus infection (chapter 7). It is suggested that this was caused by enhanced NK activity.

The exact mechanism underlying immune modulatory effects of prebiotic oligosaccharides are unknown, but are often attributed to the changes in the gut microbiota; especially to specific increases in intestinal bifidobacteria and lactobacilli. However, direct correlations between effects on the gut microbiota and the immune system have not been described widely in literature. A significant correlation between cecum lactobacilli and DTH responses was found in mice supplemented with scGOS/lcFOS/pAOS. However, the correlation was not strong and the results suggested that other factors played a role as well (chapter 5). Sonoyama et al. provided evidence that strongly suggested the existence of direct, microbiota-unrelated immune modulatory effects of oligosaccharides in vivo. In a rat model of allergic airway inflammation, eosinophilia was reduced after intraperitoneal application of $\alpha$-GOS, as well as after dietary application in cecectomized mice that were treated with antibiotics (1). These data, together with immune modulatory effects that were previously observed, prompted us to test whether there is evidence for direct immune modulatory effects of scGOS/lcFOS or pAOS in vitro, thereby excluding potential microbiota-related effects. The influence on $\mathrm{T}$ cell and dendritic cell (DC) function were investigated, because these cell types are of major importance in $\mathrm{T}$ cell-mediated immune responses and the Th1/Th2 balance. In addition, the influence of the specific oligosaccharides on NK activity was analyzed.

\section{Materials and methods}

\section{Peripheral blood mononuclear cells}

Human peripheral blood mononuclear cells (PBMC) from buffy coats, originating from healthy adult donors, were obtained from the blood bank (Sanquin, Amsterdam, the Netherlands). PBMC were isolated using Ficoll-Paque (Amersham Biosciences, Diegem, Belgium) density centrifugation and stored in liquid nitrogen. Before use, cells were thawed quickly and warm culture medium was added slowly and gently to the cells. 
Culture medium consisted of RPMI-1640 (Life Technologies, Merelbeke, Belgium) supplemented with $10 \%$ heat inactivated FCS (Perbio, Etten Leur, the Netherlands) and $100 \mathrm{U} / \mathrm{ml}$ penicillin/streptomycin). Cells were washed and centrifuged twice, were counted (Coulter Z2, Beckman Coulter, Mijdrecht, the Netherlands) and diluted to the proper concentration for use. The various in vitro experiments were performed on multiple donors to analyze the variability of the observed effects.

\section{Oligosaccharide solutions}

Stock solutions of up to $40 \mathrm{mg} / \mathrm{ml} \mathrm{scGOS} / \mathrm{lcFOS}$ and pAOS were made in culture medium. The $\mathrm{pH}$ of the $\mathrm{pAOS}$ stock solution was neutralized using sodium hydroxide. The scGOS/lcFOS powder consisted of the active ingredients spray-dried scGOS (Vivinal GOS, Borculo Domo, Zwolle, The Netherlands;) and lcFOS (Raftiline HP, Orafti, Wijchen, The Netherlands;) in a 9:1 ratio (Immunofortis). The scGOS/lcFOS powder that was used for these experiments also contained carrier and rest carbohydrates (maltodextrin, glucose and galactose). If immune modulatory effects were observed in vitro, these carbohydrates were to be tested separately to ascertain that the activity was due to the active ingredients only. Pectin-derived pAOS powder was kindly provided by Südzucker AG (Mannheim, Germany). The solutions were filter-sterilized before use (pore size $0.2 \mu \mathrm{m}$ ). Various concentration ranges of the oligosaccharide solutions were tested, as indicated in the results section.

\section{TNF- $\alpha$ production assay}

PBMC ( $1^{*} 10^{5}$ cells/well) were incubated with a wide concentration range of the oligosaccharide solutions during 24 hours under standard culture conditions (cells suspended in culture medium, incubated at $37{ }^{\circ} \mathrm{C}$ in an atmosphere containing $5 \%$ $\mathrm{CO}_{2}$ ). As a positive control, $50 \mathrm{ng} / \mathrm{ml}$ LPS was added to the culture medium. After incubation, culture supernatants were collected and stored at $-80^{\circ} \mathrm{C}$ until measurement.

\section{Mitogen proliferation assay}

Concanavalin-A (ConA, Sigma Aldrich, Zwijndrecht The Netherlands), a $\mathrm{T}$ cell mitogen, was used to induce proliferation in PBMC cultures $\left(1^{*} 10^{5}\right.$ cells/well) at 1.25 and $10 \mu \mathrm{g} / \mathrm{ml}$ (sub-optimal and optimal concentrations; determined in previous experiments; data not shown). Oligosaccharide solutions were added to the culture medium in a wide concentration range. Parallel plates were used to measure proliferation and cytokine production. The cells were incubated in a total volume of 200 
$\mu \mathrm{l}$ for $44-48$ hours. For the proliferation assay, $0.4 \mu \mathrm{Ci}{ }^{3} \mathrm{H}$-Thymidine was added per well for the last 16 hours of culture. Cells were harvested onto filterplates and ${ }^{3} \mathrm{H}$ Thymidine incorporation was measured with the aid of a scintillation counter (Wallac Microbeta 1450, Perkin Elmer, Zaventem, Belgium). The results were shown as radioactive counts per minute $(\mathrm{cpm})$ per well. For cytokine measurements, culture supernatants were harvested after $44-48$ hour incubation and stored at $-80{ }^{\circ} \mathrm{C}$ until measurement.

\section{Natural Killer cell activity assay}

Natural Killer (NK) cell activity was tested using a time-resolved fluorometric assay. PBMC (effector cells) were cocultured with K562 cells (target cells, chronic myelogenous leukemia (CML) cells), labeled with BATDA (DELFIA BATDA Reagent, Perkin Elmer Life Sciences) at Effector : Target (E:T) ratios of 50:1, 25:1, and 12.5:1. One day before the NK activity assay was performed, 3.3*105 K562 target cells were seeded in a T75 culture flask. On the day of the assay, these target cells were washed, diluted to a concentration of $1^{*} 10^{6}$ cells/ml and labeled with BATDA reagent $(12,5 \mu \mathrm{l}$ BATDA in $10 \mathrm{ml}$ cell suspension) for 15 minutes at room temperature (RT). Cells were washed 5 times with $30 \mathrm{ml}$ culture medium to remove all unbound label.

PBMC were pipetted in a U-bottom 96-well plate at the appropriate concentrations for the E:T ratios and $5^{*} 103$ target cells were added. The oligosaccharides were added to the cell cultures before the start of the 4 hour incubation period. Alternatively, oligosaccharides were preincubated with the effector cells for 20 hours, followed by the standard 4 hour incubation with the target cells. Afterwards, $20 \mu$ l supernatant was harvested into a white 96-well plate (PolySorp F96 Nunc, VWR International, Roden, The Netherlands), $180 \mu$ l europium solution (DELFIA, Perkin Elmer Life Sciences) was added and the plates were incubated in the dark for 15 minutes at RT. Time-resolved fluorescence was measured with a fluorometric analyzer (Wallac Victor2 1420 Multilabel HTS Counter, Perkin Elmer Life Sciences).

For determination of the spontaneous lysis, target cells were incubated with $100 \mu \mathrm{l}$ culture medium instead of effector cells. Maximum release was measured by incubating the target cells with $100 \mu \mathrm{l}$ culture medium with 4\% Triton X-100 (SigmaAldrich Chemie, Zwijndrecht, The Netherlands), which induces cell lysis. Data were expressed as the percentage of target cell lysis calculated by the following formula: [(experimental release - spontaneous release) / (maximum release - spontaneous release)] $x 100$. 


\section{Generation of monocyte-derived dendritic cells}

Dendritic cells were generated from adherent mononuclear cells in human blood according to established standard procedures (2). The PBMC were plated out in $5 \mathrm{ml}$ culture flasks at $2-4^{*} 10^{6}$ cells $/ \mathrm{ml}$. They were stored for 2 hours in the incubator to allow the monocytes to adhere to the flask. After 2 hours, the culture medium was removed and the non-adherent cells were washed off with PBS. The adherent cells were cultured for 5-6 days in $5 \mathrm{ml}$ RPMI with $1000 \mathrm{U} / \mathrm{ml}$ rIL-4 (CLB, Amsterdam The Netherlands) and $100 \mathrm{ng} / \mathrm{ml}$ GM-CSF (Leucomax, Novartis, Basel, Switzerland) to induce differentiation into monocyte-derived DCs. After differentiation, the cells were matured for 2 more days with $100 \mathrm{ng} / \mathrm{ml}$ LPS (positive control) or various concentrations of oligosaccharides. DC differentiation and maturation was analyzed by flow cytometer. In experiments testing pAOS, culture supernatants were harvested after maturation. In addition, pAOS was co-incubated with a sub-optimal dose of LPS $(1 \mathrm{ng} / \mathrm{ml})$ to investigate the effects on DC maturation.

\section{Flow Cytometry}

Cells were washed twice using PBS with $1 \%$ BSA. The fluorochrome-conjugated mAbs were added at saturating concentrations for $30 \mathrm{~min}$ at $4^{\circ} \mathrm{C}$, and two additional washes were performed. Cell surface expression was evaluated by single or multiple immunofluorescence staining, and analysis was performed using a Coulter Epics XLMCL flow cytometer and Expo 32 software (Beckman Coulter). These monoclonal mouse anti-human antibodies used: CD1a-FITC (Biosource); HLA-DR,DP,DQ-FITC (Pharmingen, Becton Dickinson); CD1a-PE, CD-14-PE, CD83 andCD86 (Immunotech, Beckman Coulter).

Monocyte-derived DC differentiation was confirmed by the absence of CD-14 and presence of CD1a expression (data not shown). Propidium iodide staining was used to exclude dead cells. For all DC maturation analyses, gating was used to analyze only large, living $\mathrm{CD} \mathrm{a}^{+}$cells. Expression of the maturation marker CD83 is shown as the percentage of positive cells. MHC class II and CD86 are (co)stimulatory molecules that are expressed on both immature and mature DCs, but the level of expression increases upon maturation. Therefore, the results for these markers are expressed as the mean fluorescence intensity per cell.

\section{Cytokine measurements}

High-binding capacity 96-well plates (Costar, Corning, Schiphol-Rijk, the Netherlands) were coated with the primary antibody $\left(5 \mu \mathrm{g} / \mathrm{ml}\right.$ in PBS, for 1 hour at $37^{\circ} \mathrm{C}$ or o/n at 4 
${ }^{\circ} \mathrm{C}$ ). After coating, the plates were washed 3 times with wash buffer (PBS, 0,005\% Tween) and blocked 1 hour with block buffer (PBS, 1\% BSA). After 3 washing steps, samples and standards were incubated for 2 hours at room temperature. After 5 washing steps, the biotin-conjugated secondary antibody was added at $0.5 \mu \mathrm{g} / \mathrm{ml}$ (diluted in wash buffer) and incubated for 1 hour at room temperature. Streptavidin/HRP (Biosource, Camarillo USA, Lotnr: 4401) was diluted 1:20000 in wash buffer, added and incubated for 30 minutes. TMB substrate (Pierce, Rockford USA, Lotnr: DG728591) was added and color development was allowed for a maximum of 20 minutes. The reaction was stopped with $10 \% \mathrm{H}_{2} \mathrm{SO}_{4}$ and the samples were measured at $450 \mathrm{~nm}$ using a spectrophotometer (Bio-Rad Ultramark Microplate imaging system).

For the measurement of IL-2, anti-human IL-2 (Pharmingen, cat.nr. 555051, Becton Dickinson, Breda, the Netherlands) was used as the primary antibody and biotinconjugated anti-human IL-2 (Pharmingen, cat. nr. 555040) as the secondary. For TNF- $\alpha$, the human TNF- $\alpha$ CytoSet (Biosource, Invitrogen, Breda, the Netherlands) antibody pair was used. For the measurement of IL-12p70, the IL-12p70 Eli-pair kit (CLBSanquin, Amsterdam, the Netherlands) was used.

\section{Statistical analysis}

Data were analyzed using SPSS 15.00 and Graphpad Prism 4.03 software and expressed as averages \pm standard deviation. Differences between one control and multiple test groups were analyzed using the two sided Dunnett T-test. Differences were regarded significant at $\mathrm{p}<0.05$. The in vitro assays that are described in this chapter were performed repeatedly on PBMC from different donors. The results from different donors were consistent unless indicated otherwise; representative results are shown from a single donor per type of assay.

\section{Results}

\section{TNF- $\alpha$ production}

To test whether the oligosaccharide solutions induced stimulatory effects on monocytes or were contaminated with endotoxin, PBMC TNF- $\alpha$ production was measured after 24 hour of incubation with the oligosaccharides. A concentration range of $10 \mathrm{mg} / \mathrm{ml}$ to 10 $\mathrm{pg} / \mathrm{ml}$ was tested using 10-fold dilutions, as shown in Figure 1. The oligosaccharides did not stimulate TNF- $\alpha$ production compared to the negative control $(152 \pm 32 \mathrm{pg} / \mathrm{ml})$, except for the highest dose of scGOS/lcFOS which induced intermediate concentrations 


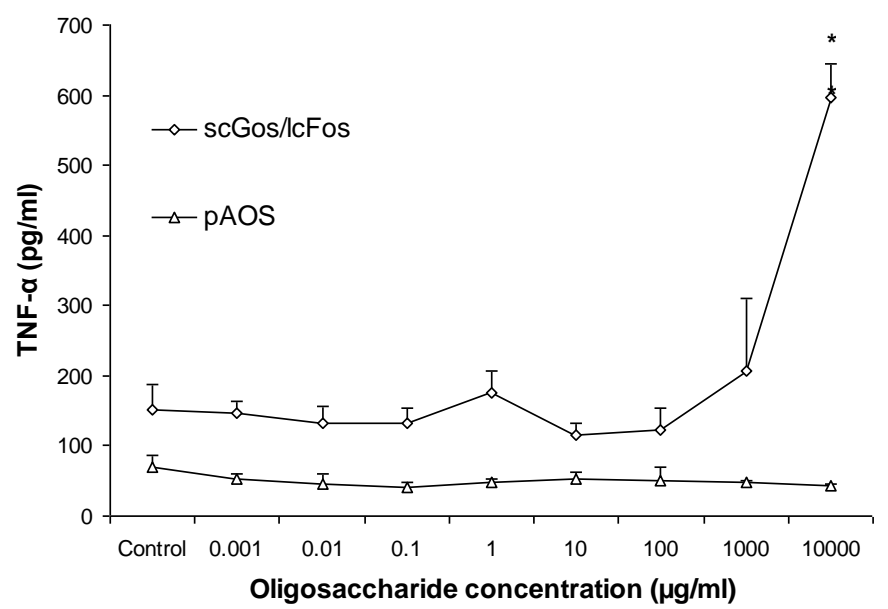

Figure 1. scGOS/lcFOS and pAOS do not induce TNF- $\alpha$ production over a broad concentration range. Oligosaccharide-induced spontaneous TNF- $\alpha$ production by human PBMC was measured, as a functional control assay for endotoxin contamination of the oligosaccharides, or to detect monocyte activation effects. Two different PBMC donors were used for the dose response curves. PBMC from these donors that were stimulated with $50 \mathrm{ng} / \mathrm{ml}$ LPS produced high amounts of TNF- $\alpha$ (>2400 pg/ml). Data are represented as means of triplicate samples. Error bars indicate the standard deviation and * indicates a significant difference compared to the unstimulated control samples $(p<0.05)$.

of TNF- $\alpha(597 \pm 48 \mathrm{pg} / \mathrm{ml} ; \mathrm{p}<0.01)$. LPS stimulation of the PBMC induced high levels of TNF- $\alpha$ production ( $>2400 \mathrm{pg} / \mathrm{ml}$ in all PBMC donors that were used). No effects on TNF- $\alpha$ production were observed with the same dose range of a 1:1 mixture of both oligosaccharide solutions (data not shown).

\section{Mitogen-induced proliferation and cytokine production}

The effects of the same dose range of oligosaccharides were tested on mitogen-induced $\mathrm{T}$ cell proliferation and cytokine production. Background proliferation of the unstimulated PBMC was $530 \pm 60 \mathrm{cpm}$. Sub-optimal and optimal ConA concentrations induced high rates of proliferation $\left(7.8 \pm 1.6{ }^{*} 10^{3} \mathrm{cpm}\right.$ and $2.4 \pm 0.5{ }^{*} 10^{4} \mathrm{cpm}$, respectively). Co-incubation with oligosaccharides did not affect the proliferative responses of ConA-stimulated PBMC (Figure 2a), except for the two highest concentrations of $\mathrm{pAOS}$ that significantly inhibited proliferation $(\mathrm{p}<0.01$ for both concentrations of pAOS and ConA). Unexpectedly, the neutralized pAOS stock solution caused acidification of culture medium at these concentrations. A 1:1 mixture of the oligosaccharides induced similar effects as pAOS alone. The proliferation was inhibited at the highest concentrations, due to acidification of the culture medium (data not shown). 

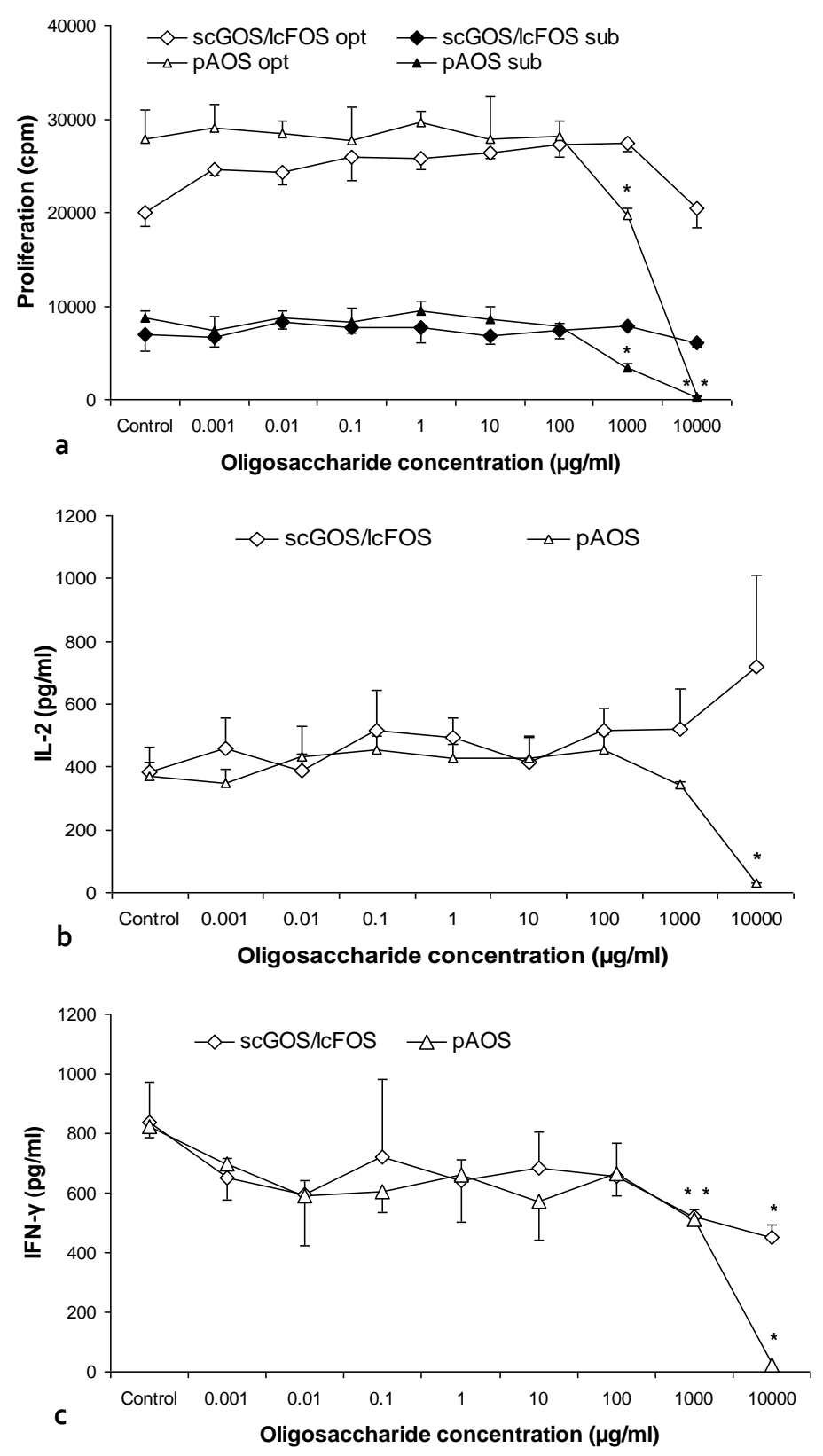

Figure 2. scGOS/IcFOS and pAOS do not affect mitogen-induced proliferation or IL-2 production over a broad concentration range. (a) Human PBMC were stimulated with sub-optimal (sub) or optimal (opt) concentrations of ConA (1.25 and $10 \mu \mathrm{g} / \mathrm{ml}$, closed and open markers, respectively). (b) IL-2 and (c) IFN- $\gamma$ production by human PBMC, stimulated with $10 \mu \mathrm{g} / \mathrm{ml} \mathrm{ConA}$. Data are represented as means of triplicate samples. Error bars indicate the standard deviation and * indicates a significant difference compared to the unstimulated control samples $(p<0.05)$. 
The effects on ConA-induced IL-2 production were similar to the effects on proliferation (Figure 2b): no oligosaccharide-induced effects were observed, except for a significant reduction at the highest concentration of pAOS $(p<0.01)$. IFN- $\gamma$ concentrations after optimal ConA stimulation was measured once (Figure $2 c)$. It was not affected over the largest part of the dose range, but it was reduced significantly compared to the control cells at the two highest concentrations of scGOS/lcFOS $(\mathrm{p}<0.05$ at $1 \mathrm{mg} / \mathrm{ml} ; \mathrm{p}<0.01$ at $10 \mathrm{mg} / \mathrm{ml}$ ) and pAOS ( $<<0.01$ at both concentrations). The inhibitory effect of high concentrations of scGOS/lcFOS was not significant compared to the lowest concentrations of scGOS/lcFOS, suggesting that the observed significance may be due to coincidental high control values. As observed with the other ConArelated parameters, the highest concentration pAOS yielded a strong inhibitory effect on IFN- $\gamma$ production. The oligosaccharides did not induce measurable spontaneous cytokine production or proliferation in PBMC without ConA.

\section{Natural killer cell activity assay}

Based on the previous experiments, doses of $50 \mathrm{ng} / \mathrm{ml}$ to $800 \mu \mathrm{g} / \mathrm{ml} \mathrm{scGOS} / \mathrm{lcFOS}$ and 5 $\mathrm{ng} / \mathrm{ml}$ to $80 \mu \mathrm{g} / \mathrm{ml}$ pAOS were tested in the NK-activity assay. At these doses, no TNF$\alpha$ production or viability problems had been observed and no spontaneous cell lysis of the K562 target cells occurred. As expected, the NK activity of thawed PBMC was proportional to the E:T ratio, but scGOS/lcFOS and pAOS did not show clear dosedependent effects within the dose ranges that were tested (Figure 3). At several concentrations of the oligosaccharides, and especially at the E:T ratio of 12.5:1, NK activity was significantly lower than in the control samples. However, the doseresponse relationships and repeated measurements indicated that this was caused by assay variation.

\section{Dendritic cell maturation}

Incubation of immature DCs with concentrations up to $200 \mu \mathrm{g} / \mathrm{ml}$ of scGOS/lcFOS (not shown) or a 1:1 mix of scGOS/lcFOS and pAOS for 48 hours did not induce enhanced expression of maturation or costimulatory markers. LPS, the positive control, clearly induced maturation as shown by an increase in the number of CD83+ cells and the expression level of MHC class II and CD86 molecules, as shown in Figure 4. pAOS appeared to enhance expression of CD83 and MHC class II in two experiments, but this was not observed in repeat experiments or with the combination of scGOS/lcFOS and pAOS. As an additional measure of maturation, it was tested whether pAOS (up to 100 $\mu \mathrm{g} / \mathrm{ml}$ ) induced IL-12p70 production in DCs, a cytokine that drives the generation of 

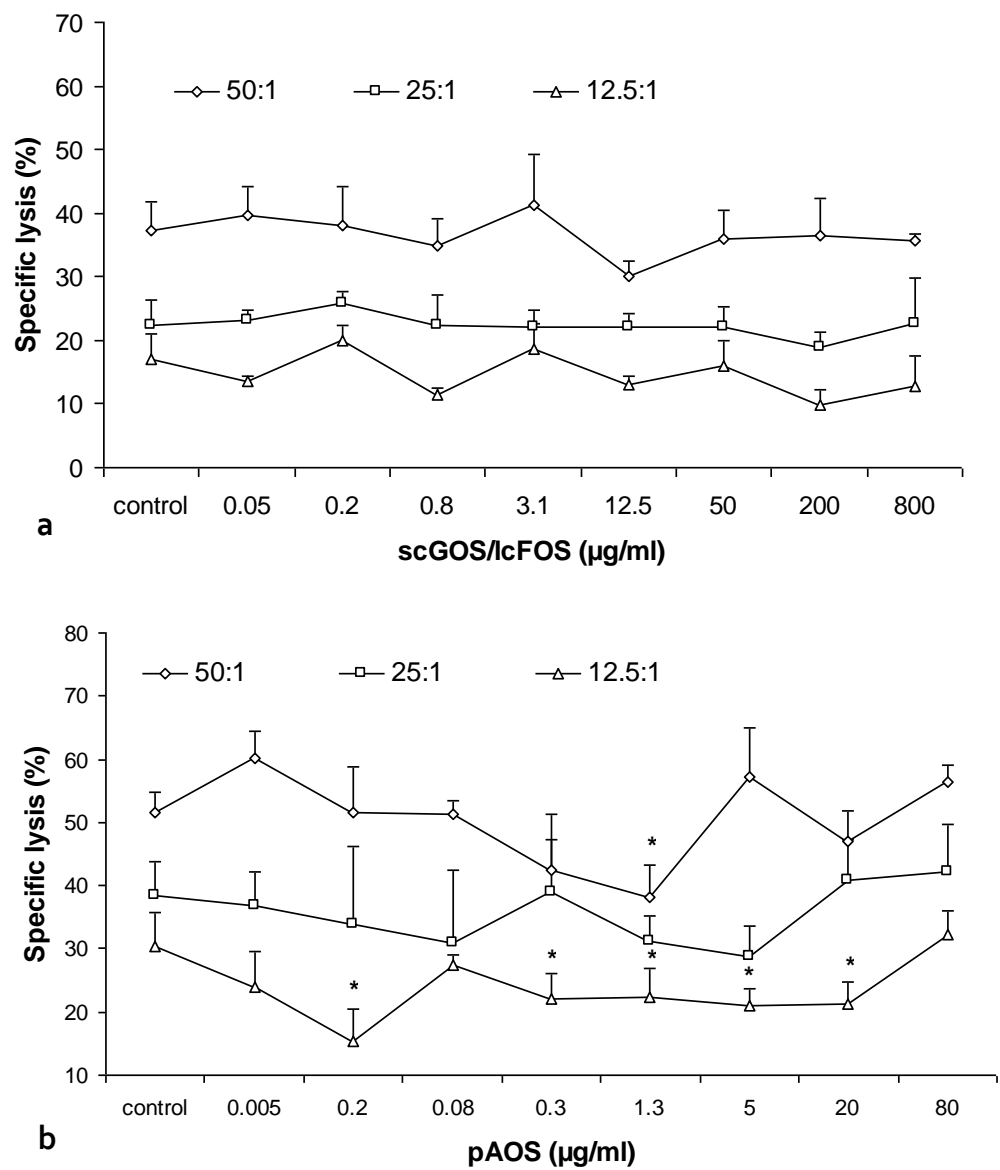

Figure 3. NK-activity of human PBMC is not affected consistently by increasing doses of scGOS/lcFOS and pAOS. Human PBMC (effector cells) were incubated for 4 hours with labeled, NK-sensitive $\mathrm{K}_{5} 62$ cells (target cells). NK-activity of PBMC was measured by the specific lysis of the $\mathrm{K}_{5} 62$ target cells over the 4 hour incubation period, as indicated on the $y$-axis. Three E:T ratios were tested: 50:1 (diamonds), 25:1 (squares) and 12.5:1 (triangles). Data are represented as means of quadruple samples. Error bars indicate the standard deviation and * indicates a significant difference compared to the unstimulated control samples at the corresponding E:T ratio $(p<0.05)$.

Th1 responses (3). However, whereas LPS-matured DCs produced low levels of IL12 p70 (67 $\pm 10 \mathrm{pg} / \mathrm{ml})$, no IL-12p70 was detectible in the supernatants of immature DCs and DCs incubated with pAOS.

Possibly, the effect of pAOS on DC maturation may have been too subtle to detect repeatedly. Therefore, it was investigated whether an effect of pAOS could be detected in the presence of a sub-optimal maturation factor. As shown in Figure 5, $1 \mathrm{ng} / \mathrm{ml} \mathrm{LPS}$ induced sub-optimal levels of CD83 and MHC class II expression, but pAOS co- 

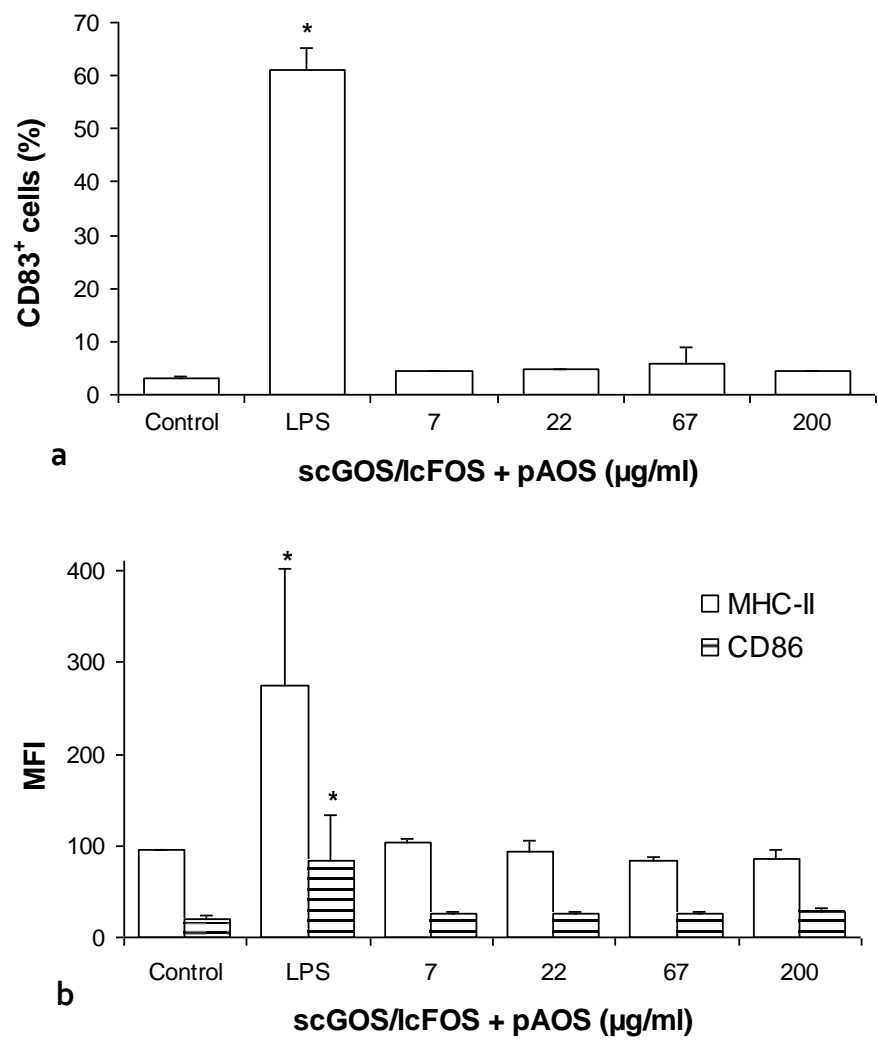

Figure 4. A combination of scGOS/lcFOS and pAOS did not induce maturation effects in differentiated monocyte-derived dendritic cells. The expression of the maturation marker CD83 (a), MHC class II molecules and the costimulatory marker CD86 (b) was measured by flow cytometry after a two-day incubation period of $\mathrm{CD}_{1} \mathrm{a}^{+}$, immature, monocyte-derived DC with various doses of oligosaccharides. Data are represented as means of duplicate samples. Error bars indicate the standard deviation and * indicates a significant difference compared to the control samples $(p<0.05)$.

incubation did not modulate DC maturation in the presence of LPS. CD86 expression appeared to be reduced at lower concentrations of pAOS, however, this effect was not significant.

\section{Discussion}

No consistent effects of scGOS/lcFOS or pAOS have been observed on the in vitro assays that were performed. scGOS/lcFOS induced moderate levels of TNF- $\alpha$ production from PBMC at $10 \mathrm{mg} / \mathrm{ml}$, but it is unclear whether this was caused by a 

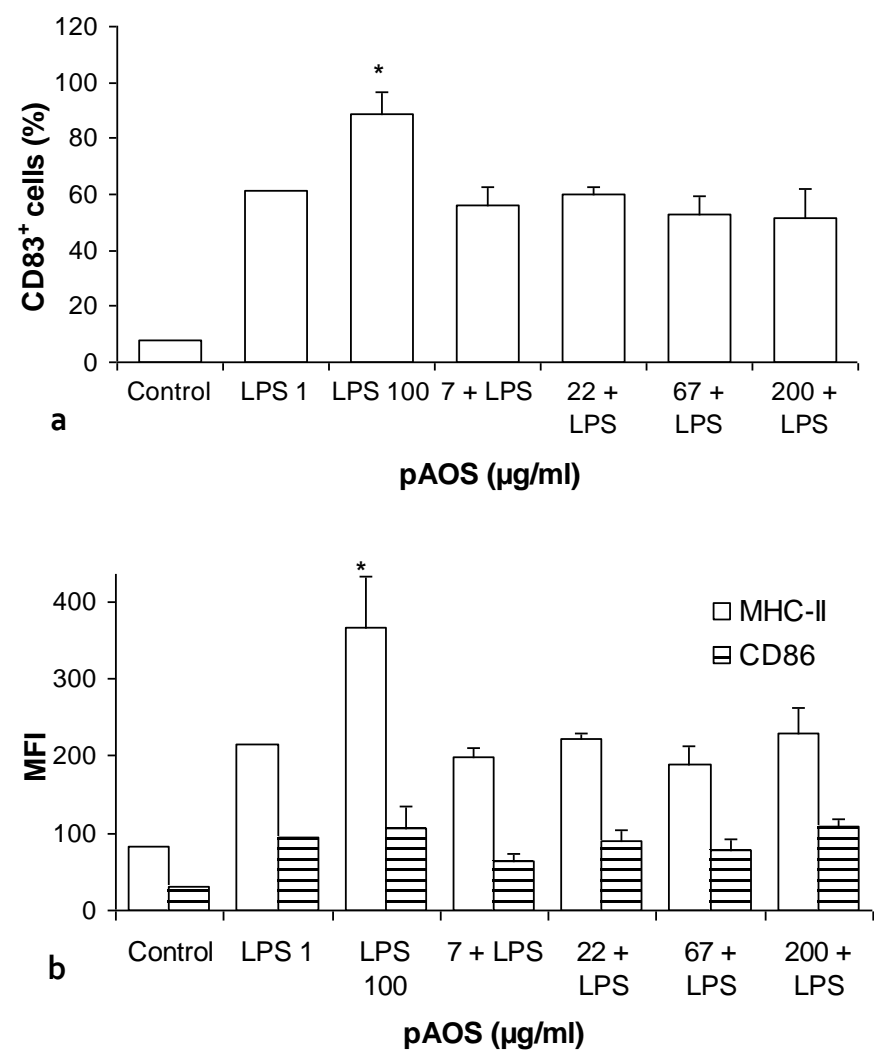

Figure 5. pAOS does not affect parameters of DC maturation in combination with a sub-optimal concentration of LPS. A sub-optimal concentration of LPS $(1 \mathrm{ng} / \mathrm{ml})$ induced intermediate expression of maturation and costimulatory markers compared to an optimal concentration ( $100 \mathrm{ng} / \mathrm{ml}$ ). Immature monocyte-derived DCs were incubated for 2 days with various concentrations of pAOS, together with the suboptimal concentration of LPS. The expression of the maturation marker CD83 (a), MHC class II molecules and the costimulatory marker CD86 (b) was measured by flow cytometry. Data are represented as means of duplicate samples. Error bars indicate the standard deviation and * indicates a significant difference compared to all samples with sub-optimal LPS concentrations $(p<0.05)$.

low-level contamination or to specific effects of the oligosaccharides. In addition, pAOS inhibited ConA responses at 1 and especially $10 \mathrm{mg} / \mathrm{ml}$, because of acidification of the culture medium. As a result, cell viability was apparently reduced, which led to reduced cell proliferation and cytokine production. These effects do not appear to be relevant as such concentrations are unlikely to be reached in vivo.

It has been described that minor amounts of scFOS ( $\mathrm{dp} \leq 4)$ were excreted in the urine of supplemented healthy male volunteers. Around $0.1 \%$ of the oral intake was recovered, indicating that at least that amount of the oligosaccharides must have been taken up from the gut into the circulation (4). In previous animal experiments, 
oligosaccharide doses of 1 to $5 \%$ were shown to induce immune modulatory effects. The average daily dietary intake in these mice was approximately $2.5 \mathrm{~g}$ (chapter 4), leading to an intake of up to $125 \mathrm{mg}$ /day. If it is assumed that the intestinal uptake in rodents is similar to humans, no accumulation of systemic oligosaccharides occurred and the blood volume of the mice was $2.5 \mathrm{ml}$ (10\% of the body weight), the maximal systemic concentration would have been below $50 \mu \mathrm{g} / \mathrm{ml}$. Consistent with this estimate, no oligosaccharides could be measured in the serum of supplemented animals using an HPLC analysis method with a limit of quantification around 1-10 $\mu \mathrm{g} / \mathrm{ml}$ (unpublished data). In relation to the estimated in vivo concentrations, the concentrations of oligosaccharides that were tested in the in vitro assays spanned broadly around the relevant range.

The current experiments were performed on human PBMC, but the in vivo immune modulatory effects on vaccination responses were observed in mice. The results from the in vitro experiments therefore cannot be compared directly to the results from the animal experiments. However, the data from the first clinical study suggests that immune modulatory effects occur in human infants that are consistent with the effects in mice, suggesting a stimulation of Th1-related cellular immunity and an inhibition of allergic Th2-related symptoms $(5,6)$. It therefore appears that the oligosaccharideinduced immune modulatory effects are similar in mice and men.

ConA stimulation aspecifically activates the majority of $\mathrm{T}$ cells and induces costimulation-dependent $\mathrm{T}$ cell proliferation and cytokine production. Therefore, this direct mode of activation and cytokine induction makes it possible to study the general $\mathrm{T}$ cell responsiveness and cytokine production. The results showed no evidence for strong stimulatory or inhibitory effects of the oligosaccharides on $\mathrm{T}$ cell proliferation or IL-2 production, a cytokine that can be produced by Th1 and unpolarized Th0 cells. Moreover, the production of the Th1 cytokine IFN- $\gamma$ was not enhanced by the oligosaccharides. Because the aspecific mechanism of ConA-induced $\mathrm{T}$ cell activation is not suited to study Th1 or Th2 polarization of naïve T cells, it is recommended to study the potential effects of the oligosaccharides in more specific assays of $\mathrm{T}$ cell activation and polarization.

Dendritic cells are central in T cell-mediated immune responses; they integrate signals from their surroundings, interact with naïve $\mathrm{T}$ cells to control their activation state and drive their polarization into Th1 or Th2 cells (7). The results indicated that the oligosaccharides did not directly induce maturation in monocyte-derived DCs. However, it is possible that the effects of the oligosaccharides may differ between DC subsets. Therefore, interactions between oligosaccharides and intestinal DCs in vivo 
might lead to different results than observed in vitro, as intestinal DCs display a different phenotype and functionality than monocyte-derived DCs (8).

No effects were observed on NK activity of PBMC when oligosaccharides were added directly to the 4-hour cytotoxicity assay. It is possible that a longer incubation time is needed to detect a potential effect on NK activity. However, since overnight preincubation of PBMC in culture medium with or without oligosaccharides inhibited the NK activity of the cells (data not shown), this could not be tested properly.

Previous animal experiments suggested that the oligosaccharides modulated the Th1/Th2 balance of vaccination responses around the priming phase of the immune response (chapter 5). Moreover, the first results from clinical trials are also consistent with modulation of the Th1/Th2 balance. However, the currently used set of in vitro assays did not cover all relevant $\mathrm{DC}$ and $\mathrm{T}$ cell functions to investigate potential modulation of the Th1/Th2 balance. Cytokine production by DCs may play an important role, which could be analyzed using differentiated and matured DCs that are stimulated with CD40-ligand. In addition, $\mathrm{T}$ cell activation and polarization of naïve $\mathrm{T}$ cells could be studied by measuring intracellular cytokine production of IL- 4 and IFN- $\gamma$ after activation. However, in order to be able to detect oligosaccharide-induced effects on the Th1/Th2 balance, any effects on DCs, T cells or their interaction could be relevant. Therefore, it is recommended to test the oligosaccharides in a DC-T cell interaction model, using naïve $\mathrm{T}$ cells that are stimulated with a superantigen or in an antigen-specific manner $(9,10)$. Using such an integrated approach, any effects on DCs and/or $\mathrm{T}$ cells that influence the Th1/Th2 balance can be detected by measuring intracellular T cell cytokines IL-4 and IFN- $\gamma$ at the end of the experiments.

Other non-digestible carbohydrates (inulin and $\beta$-glucans) have been shown fairly recently to activate IFN- $\gamma$ primed macrophages or macrophage-like cells $(11,12)$. In addition, $\alpha$-linked GOS has been described to increase spontaneous and LPS-induced inflammatory cytokine production in alveolar macrophages (13). Although no spontaneous inflammatory cytokine production by monocytes or other PBMC was observed except for the highest concentration of scGOS/lcFOS, it is recommended that additional experiments are carried out to study the effects of the oligosaccharides on normal or IFN- $\gamma$ primed macrophages.

In conclusion, no evidence was found in the present study for direct, microbiotaunrelated immune modulatory effects of the scGOS/lcFOS and/or pAOS oligosaccharides in vitro. However, these results are not conclusive about the relevance of direct, microbiota-independent immune modulatory effects in vivo. The observed 
effects of the oligosaccharides in animal models and a clinical trial, consistently suggesting a shift in the Th1/Th2 balance, warrant additional experiments to study $\mathrm{T}$ cell polarization in vitro, preferably using a DC-T cell coculture model. In addition, the potential effects of the specific oligosaccharides on (IFN- $\gamma$-primed) macrophage activation and inflammatory cytokine production need further investigation.

\section{References}

1. Sonoyama, K., H. Watanabe, J. Watanabe, N. Yamaguchi, A. Yamashita, H. Hashimoto, E. Kishino, K. Fujita, M. Okada, S. Mori, S. Kitahata, and J. Kawabata, Allergic airway eosinophilia is suppressed in ovalbumin-sensitized Brown Norway rats fed raffinose and alpha-linked galactooligosaccharide. J Nutr, 2005. 135(3): p. 538-43.

2. Sallusto, F. and A. Lanzavecchia, Efficient presentation of soluble antigen by cultured human dendritic cells is maintained by granulocyte/macrophage colony-stimulating factor plus interleukin 4 and downregulated by tumor necrosis factor alpha. J Exp Med, 1994. 179(4): p. 1109-18.

Heufler, C., F. Koch, U. Stanzl, G. Topar, M. Wysocka, G. Trinchieri, A. Enk, R.M. Steinman, N. Romani, and G. Schuler, Interleukin-12 is produced by dendritic cells and mediates T helper 1 development as well as interferon-gamma production by Thelper 1 cells. Eur J Immunol, 1996. 26(3): p. 659-68.

Molis, C., B. Flourie, F. Ouarne, M.F. Gailing, S. Lartigue, A. Guibert, F. Bornet, and J.P. Galmiche, Digestion, excretion, and energy value of fructooligosaccharides in healthy humans. Am J Clin Nutr, 1996. 64(3): p. 324-8.

5. Arslanoglu, S., G. Moro, J. Schmitt, and G. Boehm, Early dietary intervention with a mixture of prebiotic oligosaccharides reduces the incidence of allergy associated symptoms and infections during the first 2 years of life. J Pediatr Gastroenterol Nutr, 2007. 44(S1): p. e217.

6. Moro, G., S. Arslanoglu, B. Stahl, J. Jelinek, U. Wahn, and G. Boehm, A mixture of prebiotic oligosaccharides reduces the incidence of atopic dermatitis during the first six months of age. Arch Dis Child, 2006. 91(10): p. 814-9.

7. Stockwin, L.H., D. McGonagle, I.G. Martin, and G.E. Blair, Dendritic cells: immunological sentinels with a central role in health and disease. Immunol Cell Biol, 2000. 78(2): p. 91-102.

8. Kelsall, B.L. and M. Rescigno, Mucosal dendritic cells in immunity and inflammation. Nat Immunol, 2004. 5(11): p. 1091-5.

9. Kikuchi, K., Y. Yanagawa, T. Aranami, C. Iwabuchi, K. Iwabuchi, and K. Onoe, Tumour necrosis factoralpha but not lipopolysaccharide enhances preference of murine dendritic cells for Th2 differentiation. Immunology, 2003. 108(1): p. 42-9.

10. Vieira, P.L., E.C. de Jong, E.A. Wierenga, M.L. Kapsenberg, and P. Kalinski, Development of Th1inducing capacity in myeloid dendritic cells requires environmental instruction. J Immunol, 2000. 164(9): p. 4507-12.

11. Berner, M.D., M.E. Sura, B.N. Alves, and K.W. Hunter, Jr., IFN-gamma primes macrophages for enhanced TNF-alpha expression in response to stimulatory and non-stimulatory amounts of microparticulate beta-glucan. Immunol Lett, 2005. 98(1): p. 115-22.

12. Koo, H.N., S.H. Hong, H.G. Seo, T.S. Yoo, K.N. Lee, N.S. Kim, C.H. Kim, and H.M. Kim, Inulin stimulates $\mathrm{NO}$ synthesis via activation of PKC-alpha and protein tyrosine kinase, resulting in the activation of NF-kappaB by IFN-gamma-primed RAW 264.7 cells. J Nutr Biochem, 2003. 14(10): p. 598-605. 
13. Abe, C., K. Fujita, E. Kikuchi, S. Hirano, H. Kuboki, A. Yamashita, H. Hashimoto, S. Mori, and M. Okada, Effects of alpha-linked galactooligosaccharide on adjuvant-induced arthritis in Wistar rats and type II collagen-induced arthritis in DBA/1 J mice. Int J Tissue React, 2004. 26(3-4): p. 65-73. 


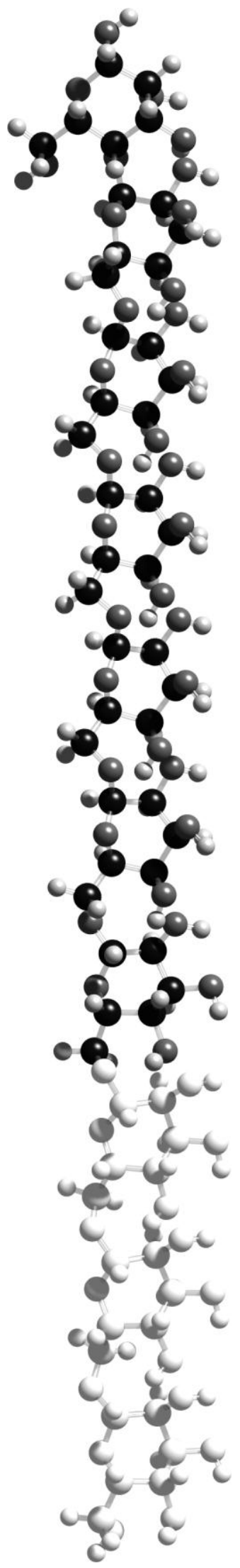

Resistance to cytomegalovirus infection in mice is enhanced by a specific mixture of dietary oligosaccharides

\author{
F. Stassen \\ A.P. Vos \\ G. Grauls \\ K. Knipping \\ A. Crienen \\ J. Garssen \\ C. Bruggeman \\ B. van 't Land
}




\section{Abstract}

Dietary supplementation with a specific prebiotic mixture of oligosaccharides was shown previously to modulate systemic vaccination responses. This prompted us to evaluate the effects on a systemic infection, using a model for murine cytomegalovirus (MCMV) infection.

C57BL/6J and BALB/c mice received supplemented or control diets and were infected with MCMV. The supplemented diet contained $2 \%(\mathrm{w} / \mathrm{w})$ of a specific mixture of shortchain galactooligosaccharides (scGOS), long-chain fructooligosaccharides (lcFOS) and pectin-derived acidic oligosaccharides (pAOS). The viral load was measured in several organs using a quantitative real-time PCR technique. Plaque assay was performed on liver homogenates to detect infectious virus at various time points. Cellular immunity to cytomegalovirus was analyzed using delayed-type hypersensitivity (DTH) responses Multiple organs showed a significant reduction in MCMV copy number one day after infection in supplemented C57BL/6J mice. Additionally, the number of mice with detectable infectious virus in the liver was reduced in the supplemented group. Increased serum levels of IFN- $\alpha$ and $-\gamma$ serum levels were found in supplemented C57BL/6J mice, suggesting that supplementation enhanced innate immune responses, leading to increased NK activity. Specific cellular responses were detectable, shown by significant MCMV-specific DTH responses in C57BL/6J mice. However, no major differences were detected between control and supplemented mice. No significant immune-modulatory or anti-viral effects of supplementation were observed in BALB/C mice.

In conclusion, this study suggests that supplementation with a specific prebiotic oligosaccharide mixture influences the onset, and possibly the severity of a systemic MCMV infection in C57BL/6J mice. This warrants further investigations into the mechanisms of action.

\section{Introduction}

Prebiotic carbohydrates are non-digestible food ingredients with potential immune modulatory properties. Local intestinal effects have been shown including modulation of cytokine production and increased B-cell cellularity in Peyer's patches, as well as enhanced intestinal IgA secretion (1-4). Systemic effects have been reported also. 
Buddington and coworkers (5) demonstrated that dietary fructans increased the resistance to bacterial infections in mice. In our previous work, a specific mixture of short-chain galactooligosaccharides (scGOS), long-chain fructooligosaccharides (lcFOS) and pectin-derived acidic oligosaccharides (pAOS) enhanced systemic, vaccine-specific delayed-type hypersensitivity (DTH) responses in C57BL/6J mice (chapters 3 and 4). This prompted us to evaluate the effects of this mixture functionally on a systemic viral infection, using a mouse model for murine cytomegalovirus (MCMV) infection.

Cytomegalovirus (CMV) is a species-specific member of the $\beta$-herpes viruses. Primary infection occurs most frequently during childhood or adolescence: in immune competent hosts, infection is usually associated with no or only mild clinical symptoms. After clearance of primary infection, the virus remains latent with episodes of endogenous reactivation. However, in immune-compromised hosts, primary infection or reactivation of CMV can lead to disease manifestations like pneumonia, gastrointestinal disease, hepatitis, or retinitis. Murine CMV infection resembles CMV infection in humans to large extent (6). The immune response after primary infection includes both humoral and cellular responses (including NK cells, macrophages and antigen-specific $\mathrm{T}$ cells). The clearance of acute $\mathrm{CMV}$ infections requires various responses in host defense, but occurs mainly by the activation of CD8+ T cells. CD4+ T cells can control the infection in the absence of CD8+ T cells. NK cells contribute to the control of CMV infection by the production of interferon- $\gamma$ (IFN- $\gamma$ ) and by direct cytotoxicity to virus-infected cells (7). Mouse strains vary in susceptibility to CMV infection; for instance BALB/c mice are susceptible whereas C57BL/6J mice are more resistant (8). This difference is mainly based on the capacity to exhibit an efficient NK cell response. The NK cell response is controlled by a single dominant locus, named Cmv1, which encodes the $\mathrm{Ly} 49 \mathrm{H}$ receptor. The $\mathrm{Ly} 49 \mathrm{H}$ receptor is present on $50 \%$ of all NK cells of MCMV-resistant C57BL6 mice but absent on NK cells of the MCMVsensitive strain Balb/C, thereby emphasizing the role of NK cells in the early control of MCMV infection (7).

In this study, the effects of dietary supplementation with a specific mixture of oligosaccharides on resistance to infection and modulation of cellular immunity were investigated using a mouse model of systemic MCMV infection, both in BALB/c and C57BL/6J strains. 


\section{Materials and methods}

\section{Mice}

Eight-week-old male specific pathogen-free (spf) inbred C57BL/6J mice (Maastricht, the Netherlands) and eight-week-old male spf BALB/c mice (Charles River, Someren, the Netherlands) were obtained and housed under standard housing conditions with a 12 $\mathrm{h}$ dark and light cycle. All animals had free access to tap water and the control or supplemented semi-purified AIN-93G diet (Research Diet Services, Wijk bij Duurstede, The Netherlands). The study protocol was reviewed and approved by the Animal Experimental Committee of the University of Maastricht.

\section{Virus stocks/infection}

The MCMV (Smith strain) stock used for inoculation was prepared by homogenization of salivary glands isolated from $\mathrm{C} 57 \mathrm{BL} / 6$ or BALB/c mice that had both been infected with $5 \times 10^{3}$ plaque-forming units (PFU) of MCMV 3 weeks before sacrifice. The MCMV stock used for the DTH was prepared from infected confluent mouse embryo fibroblast layers derived from either C57BL/6J or BALB/C mice. Virus was harvested by centrifugation of cultures displaying 100\% cytopathic effect, and heat-inactivated for 30 $\mathrm{min}$ at $56^{\circ} \mathrm{C}$. The heat-inactivated virus stock was obtained by centrifugation for $30 \mathrm{~min}$ at $30.000 \mathrm{rpm}(65.000 \mathrm{~g})$, while the supernatant served as negative control. The presence of infectious virus was excluded by a standard plaque assay.

\section{Nutrition}

Semi-purified AIN-93G diet contains approximately 40\% cornstarch, 13\% dextrinized cornstarch, $10 \%$ sucrose and $5 \%$ cellulose, adding up to a total carbohydrate content of $68 \%$. In the supplemented diets, $2 \%$ of the oligosaccharide mixture was exchanged for the same weight of total carbohydrates in the diet. The oligosaccharide mixture consisted for $50 \%$ of a combination of scGOS and lcFOS (scGOS/lcFOS) and for $50 \%$ of pAOS. The mixture was blended with the AIN-93G diet and pressed into pellets. scGOS/lcFOS is a spray-dried powder mix of trans-Galactooligosaccharides (Vivinal GOS, Borculo Domo, Zwolle, The Netherlands) and lcFOS (Raftiline HP, Orafti, Wijchen, The Netherlands), in a 9:1 ratio. It consists of approximately 51\% GOS and FOS oligosaccharides, 19\% maltodextrin, 16\% lactose and 14\% glucose. pAOS contains 
partially unsaturated and partially methylated galacturonic acids (kindly provided by Südzucker AG, Mannheim, Germany). It consists of approximately 75\% multimeric pectin-derived sugar molecules, $10 \%$ monomeric sugars, and $15 \%$ of ash and other components.

\section{Infection protocol}

At day $0, \mathrm{C} 57 \mathrm{BL} / 6 \mathrm{~J}$ and BALB/c mice were infected by intra-peritoneal injection with $5 \times 10^{3}$ or $2.5 \times 10^{3}$ PFU MCMV respectively, and sacrificed at 1, 2, 4, 6, 14 and 28 days ( $\mathrm{n}=$ 5 per time point). Dietary supplementation was started two weeks prior to infection and continued throughout the experiment. Animal weight was measured at the start of supplementation and three times weekly, until mice were sacrificed. Prior to sacrifice, mice were anaesthetized using pentobarbital (Nembutal ${ }^{\text {, }}$, Sanofi Sante B.V. Maassluis, The Netherlands) and blood was collected by left ventricular puncture. Subsequently, salivary gland, lung, liver, heart and spleen were removed and (a part from) each organ was stored directly at $-80^{\circ} \mathrm{C}$ until further processing. A second part of the liver was immediately frozen in sterile Earle's Minimal Essential Medium (EMEM) supplemented with $2 \%$ fetal calf serum until Plaque assay was performed.

\section{DNA RT-PCR}

From all organs (salivary gland, lung, liver, heart and spleen), DNA was isolated using the Wizard genomic DNA purification kit (Promega Benelux B.V., Leiden, the Netherlands) according to the recommendations of the manufacturer. MCMV genome copy numbers were measured using a MCMV specific RT-PCR (ABI Prism 7000) as described (9). In short, primers and probes for the detection of MCMV were based on the MCMV glycoprotein B sequence (gB, Genbank accession number M735191, forward primer 5'-AGGGCTTGGAGAGGACCTACA-3', reverse primer 5'GCCC GTCGGCAGTCTAGTC-3' and probe 5'-AGCTAGACGACAGCCAACGCAACGA-3'). The probe carried a 5'TAM reporter and a 3'TAMRA quencher group. To generate a standard curve serial dilutions of a known MCMV concentration were included in each run as well as a negative control, containing reagents only. Each sample was measured twice and with the average the copy number was calculated.

\section{Plaque assay of infectious MCMV}

The presence of infectious virus in the liver was determined by titration of liver homogenates as described (10). In short, confluent mouse embryo fibroblast layers 
were cultured in triplicate with liver homogenate. The day after infection at which infectious virus became detectable was recorded. Results are expressed as percentage of mice with detectable infectious virus.

\section{Serum cytokine analysis:}

Interferon- $\alpha, \beta$ and $\gamma$ responses were measured at four time-points after infection. Serum levels of IFN were measured using commercially available kits according to the manufacturers protocol; mouse IFN- $\alpha$ ELISA kit (PBL Biomedical Laboratories, Piscataway NY, USA) (Product \#42100-1) sensitivity range 10-500 pg/ml, mouse IFN- $\$$ ELISA kit (PBL Biomedical Laboratories, Piscataway NY, USA) (Product \#42400-1) sensitivity range 15.6-1000 pg/ml, and mouse IFN- $\gamma$ ELISA kit (RayBiotech, Inc., Norcross, USA) (Product \#ELM-IFN $\gamma$-001) sensitivity range 8.23-6000 pg/ml. Serum samples were serially diluted twofold, starting 1:5.

\section{MCMV-specific DTH}

MCMV-specific DTH reactions were performed as described elsewhere (11). In short, the DTH reactions were induced 1 day prior to sacrifice by subcutaneous injection of 25 $\mu \mathrm{L}$ heat-inactivated MCMV into the ear pinnae of one ear. As a control, the other ear was injected with $25 \mu \mathrm{L}$ of supernatant harvested after centrifugation (see above). Ear thickness was measured in duplicate before challenge and 24 hours afterwards (at the end of the experiment), with a digital micrometer (Mitutoyo Digimatic 293561, Veenendaal, The Netherlands). The MCMV specific DTH response was calculated by subtraction the basal ear thickness from the value at 24 hours after challenge and was corrected for control swelling.

\section{Influenza vaccination-specific DTH}

The immune-modulatory effects of the oligosaccharide mixture used in this experiment has been tested extensively in C57BL/6 mice. In this study, a vaccination experiment was performed to reproduce the previous results in a different laboratory and to make a comparison between the immune modulatory effects of the supplemented diet in C57BL/6 and BALB/c mice. Two groups of C57BL/6J and BALB/c mice ( $\mathrm{n}=5$ per group) received a primary vaccination and a booster vaccination with a human influenza subunit vaccine consisting mainly of haemagglutinin proteins of 3 different influenza strains (Influvac, Solvay Pharmaceuticals, Weesp, The Netherlands). Vaccination was performed by subcutaneous injection of a $1: 1 \mathrm{mix}$ of vaccine $(0.30 \mu \mathrm{g} / \mathrm{ml})$ and adjuvant 
(Stimune, previously known as Specol: Cedi-diagnostics, Lelystad, The Netherlands) in a total volume of $100 \mu \mathrm{L}$. The booster vaccination was given 21 days after the primary vaccination. For each mouse strain, one group of mice received GOS/FOS/AOS supplemented diet, and one group received control diet.

Vaccine-specific DTH reactions were induced 9 days after booster vaccination, by subcutaneous injection of $25 \mu \mathrm{L}$ Influvac into the ear pinnae of one ear. As a control, the other ear was injected with $25 \mu \mathrm{L}$ phosphate-buffered saline. Ear thickness was measured in duplicate before challenge, as well as 24 hours thereafter, with a digital micrometer (Mitutoyo Digimatic 293561, Veenendaal, The Netherlands). The vaccine specificity of the DTH response was calculated by subtracting the basal ear thickness from the value at 24 hours after challenge and was corrected for control swelling.

\section{Statistical analysis}

Results are expressed as means and SEM. Differences between treatments were analyzed using two-sided paired t-tests, considering $\mathrm{P}<0.05$ statistically significant. Oligosaccharides are known to augment rather than reduce DTH responses to a fixed amount of antigen, and therefore a one-sided t-test was used in statistical analysis of the influenza-vaccine response in the nutritional control experiment. Statistical analysis on viral load measurements was performed using a Wilcoxon Signed rank test to determine differences between the diets.

\section{Results}

\section{Lower MCMV copy numbers one day after infection in supplemented $C_{57} \mathrm{BL} / 6 \mathrm{~J}$ mice.}

Two weeks prior to infection, both strains of mice (C57BL/6J and BALB/c) received dietary supplementation containing the specific mixture of oligosaccharides or control, which was continued throughout the experiment. At day 0 , all mice were infected by intra-peritoneal injection and MCMV genome copy numbers were measured using a MCMV specific RT-PCR in various organs at 1, 2, 4, 6, 14 and 28 days after infection (Figure 1). In both strains, MCMV was detected in all organs, and copy numbers changed with time after infection. At day 6, all organs except the salivary gland showed maximal copy-numbers, which declined thereafter. No significant differences were observed between the supplemented and control diets in copy numbers in individual organs from BALB/C mice. In C57BL/6J mice, liver, heart, spleen, and lung harvested one day after infection from supplemented animals showed significantly 


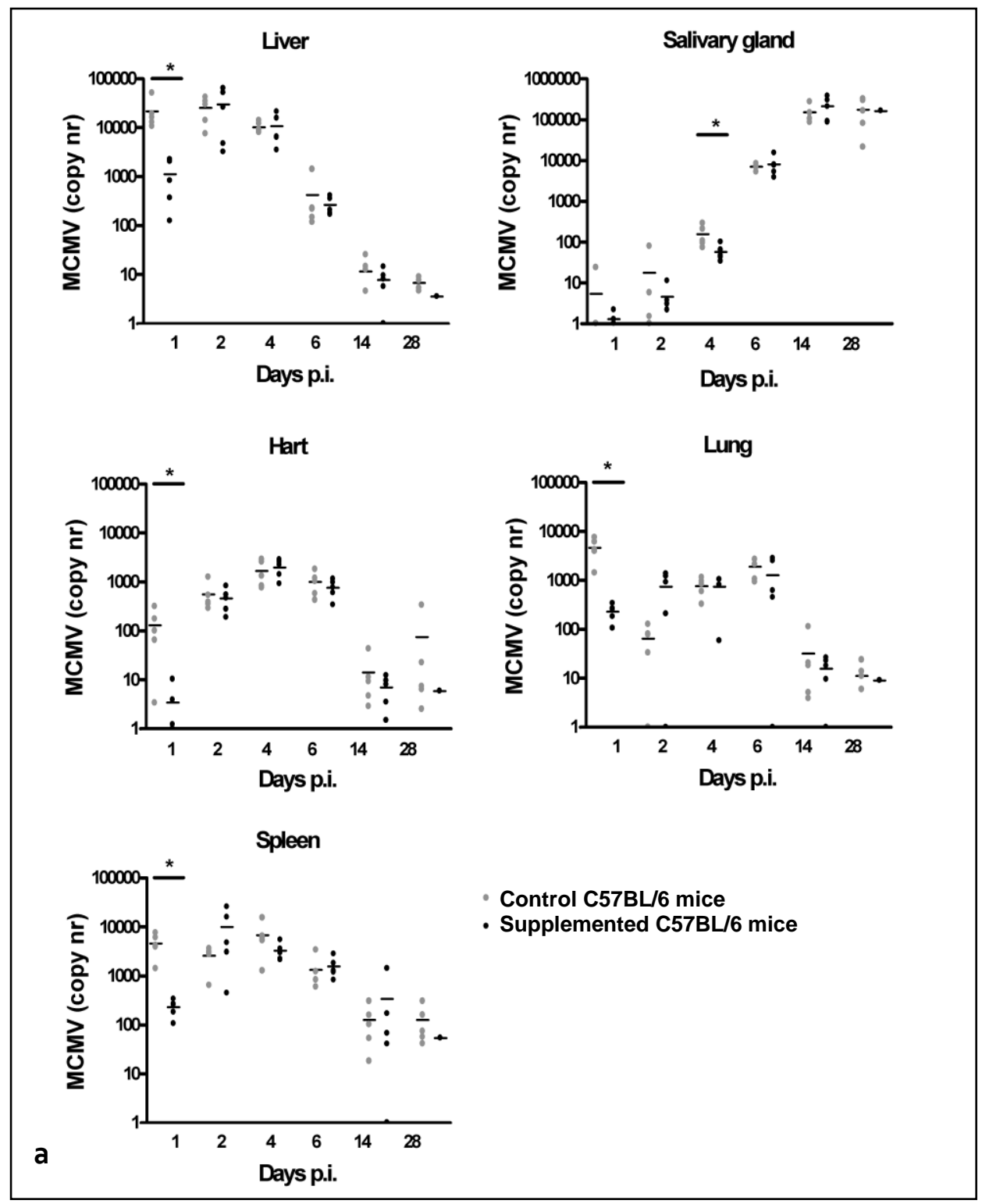

Figure 1. Differences in MCMV load between animals receiving supplemented and control diet were determined by measuring the MCMV DNA copy number. $C_{57} \mathrm{BL} / 6 \mathrm{~J}$ mice (a) and BALB/c mice (b) were divided in two groups $(n=30)$ receiving control (gray dots) or supplemented (black dots) diets, starting 2 weeks prior to infection and continued until the end of the experiment. The animals were sacrificed at indicated time points and liver, spleen, heart, lung, and salivary gland were subjected to a quantitative RT-PCR for MCMV ( $n=5$ per time point). Viral load is presented as MCMV DNA copy number per $\mu \mathrm{g}$ total DNA. Single data points are given and the geometric mean is indicated by a dash. $\left(^{*}\right)$ Indicates significant differences between intervention group and the control group at $\mathrm{P}<0.05$.

(Figure $1 \mathrm{~b}$ is printed on the next page) 


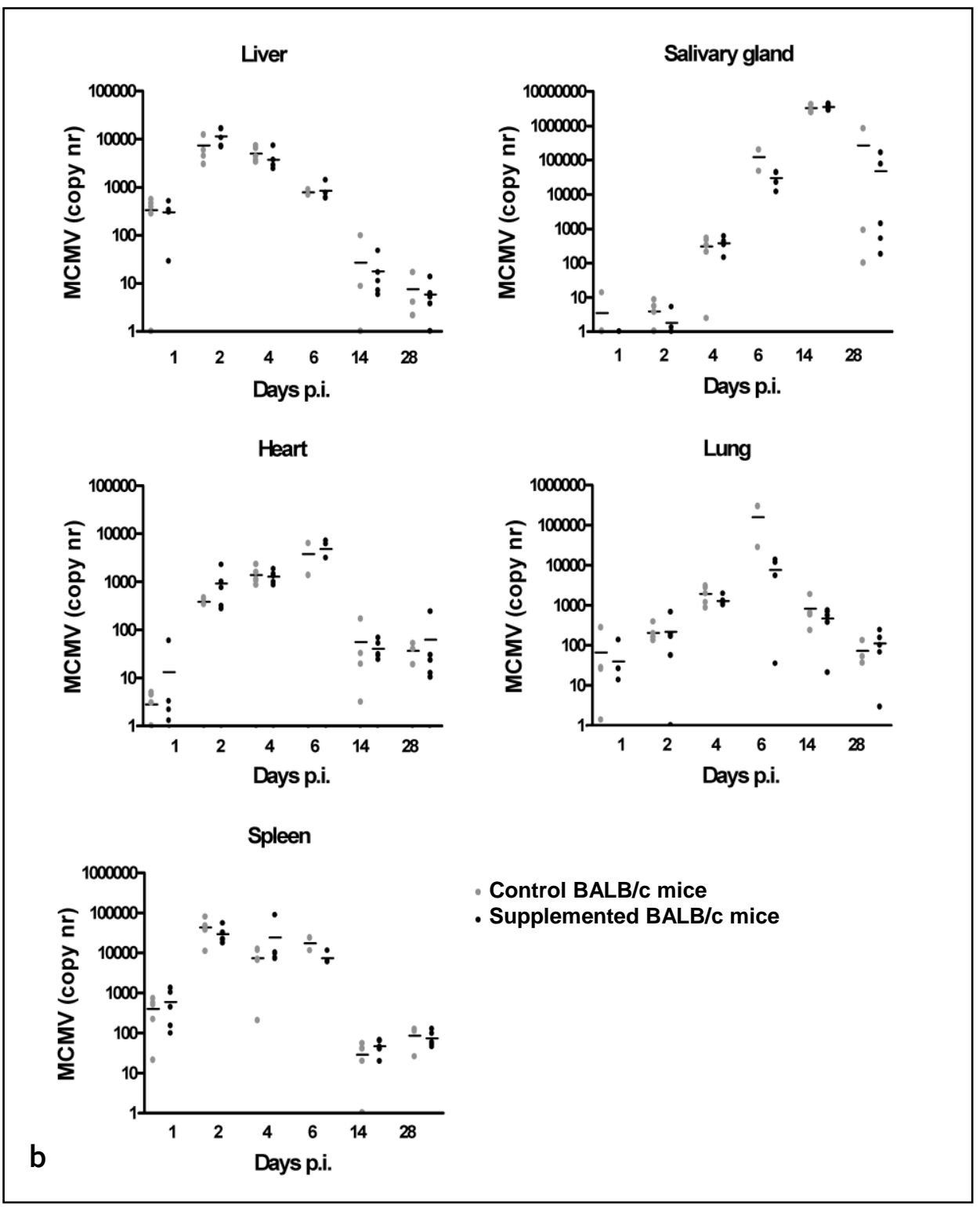

lower copy numbers than control animals $(\mathrm{p}<0.05)$. From day two onwards, no significant differences between the diets were detected. Within the salivary gland, the increase in MCMV specific copy number was delayed compared to the other organs. In supplemented C57BL/6J mice, a significant lower copy number could be detected on day $4(\mathrm{P}<0.05)$. 
Infectious virus was detectable in reduced number of supplemented C57BL/6J mice The difference between MCMV copy numbers at day one in C57BL/6J mice prompted us to measure the amount of infectious virus in the liver. The detection of infectious MCMV by plaque test was performed in the liver from C57BL/6J mice, at 1, 2, 4, and 6 days after infection ( $n=5$ per group). At all time points, the percentage of mice with a detectable amount of infectious virus was reduced in supplemented animals compared to mice receiving control diets (Table 1 ).

\section{Increased serum levels of IFN- $\alpha$ and INF- $\gamma$ in supplemented $\mathrm{C}_{57} \mathrm{BL} / 6 \mathrm{~J}$ mice}

As part of the innate immune response, IFN- $\alpha,-\beta$ and $-\gamma$ concentrations were determined in serum samples of C57BL/6J mice, at 1, 2, 4, and 6 days after infection ( $n=5$ per group). At day 2, significant levels of both IFN- $\alpha$ and IFN- $\gamma$ could be detected, which were reduced at day 4 . Increased levels of IFN- $\gamma$ and IFN- $\alpha$ were measured in supplemented C57BL/6J mice compared to control mice, although the differences were not statistically significant (Figure 2 ). No IFN- $\beta$ was detectable at any time point.

\section{MCMV-specific DTH responses}

As a parameter for cellular immunity to the virus, DTH responses to inactivated virus were measured. (Figure 3). C57BL/6J mice developed a detectable MCMV-specific DTH response, which increased with time after infection. The magnitude of the ear swelling was in the same range for supplemented and control animals, but the time at which a significant reaction was first detectable seemed to differ between the diets. In mice receiving control diets, DTH reached a significant level from day 6 onwards, whereas in supplemented mice it reached significance on day 14. The MCMV-specific DTH response in BALB/c mice remained at background levels during the course of infection, and there was no major difference between the two diets.

Table 1 Number of mice with detectable infectious virus in the liver. At time points indicated, liver from

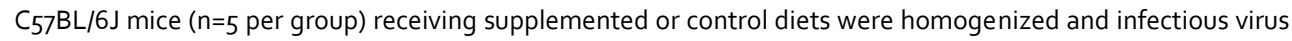
was detected using the plaque assay. The number of mice with detectable infectious virus is presented in the table.

\begin{tabular}{|c|c|c|c|c|}
\hline & Day 1 & Day 2 & Day 4 & Day 6 \\
Control & $\mathbf{3}$ & $\mathbf{5}$ & $\mathbf{2}$ & $\mathbf{1}$ \\
Suppl. & - & $\mathbf{4}$ & $\mathbf{1}$ & - \\
\hline
\end{tabular}



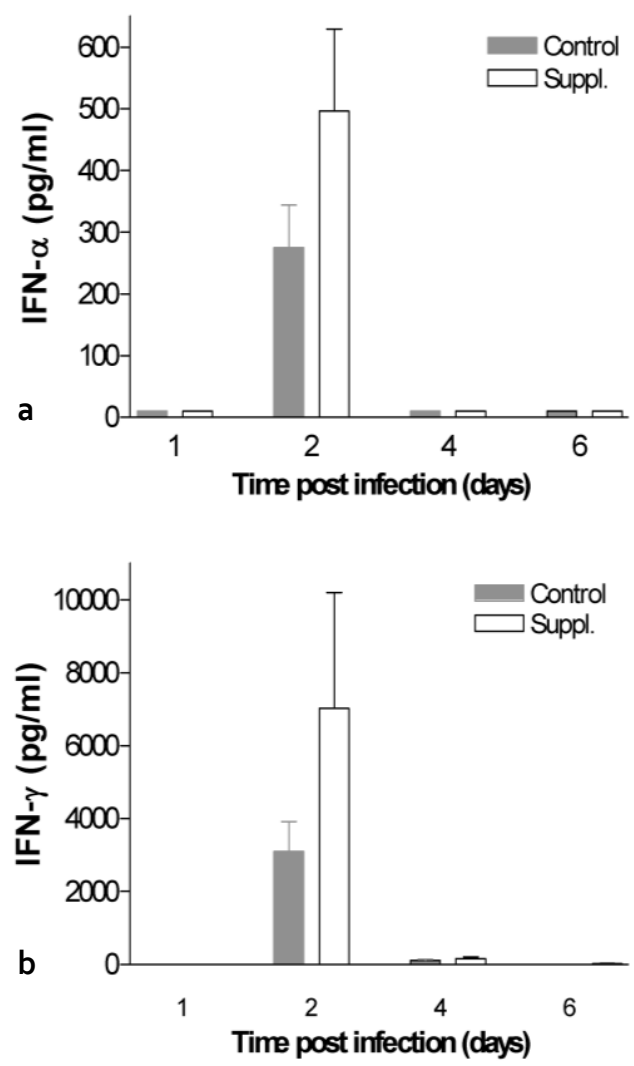

Figure 2. Effect of supplementation on IFN- $\alpha$ and $-\gamma$ concentrations in serum. Serum samples from MCMV infected $\mathrm{C}_{57} \mathrm{BL} / 6 \mathrm{~J}$ mice ( $\mathrm{n}=5$ per group) receiving supplemented (black bars) or control (gray bars) diets, were taken at time points indicated. Both IFN- $\alpha$ (a) and IFN- $\gamma$ (b) were present in serum samples after MCMV infection. Data are presented as means per group \pm SEM.

\section{Supplementation improved DTH response to vaccine in $C_{57} B L / 6 \mathrm{~J}$ but not in $B A L B / C$ mice}

In order to investigate possible differences in immune modulatory effects of the supplemented diets, a vaccination experiment was performed to measure vaccinespecific DTH responses in C57BL/6J and BALB/C mice. In both C57BL/6J and BALB/C mice ( $n=5$ per group), an influenza-specific DTH response was measured at day 28 after vaccination (Figure 4). Supplemented C57BL/6J mice showed a significantly higher response than control animals (DTH response $150 \pm 23$ vs. $88 \pm 21 \mu \mathrm{m}, \mathrm{p}<0.05$ ), indicating an improved immune response to the Flu-vaccine-antigens in C57BL/6J mice. No difference was observed in BALB/c mice: the DTH response was similar in supplemented and control animals. 

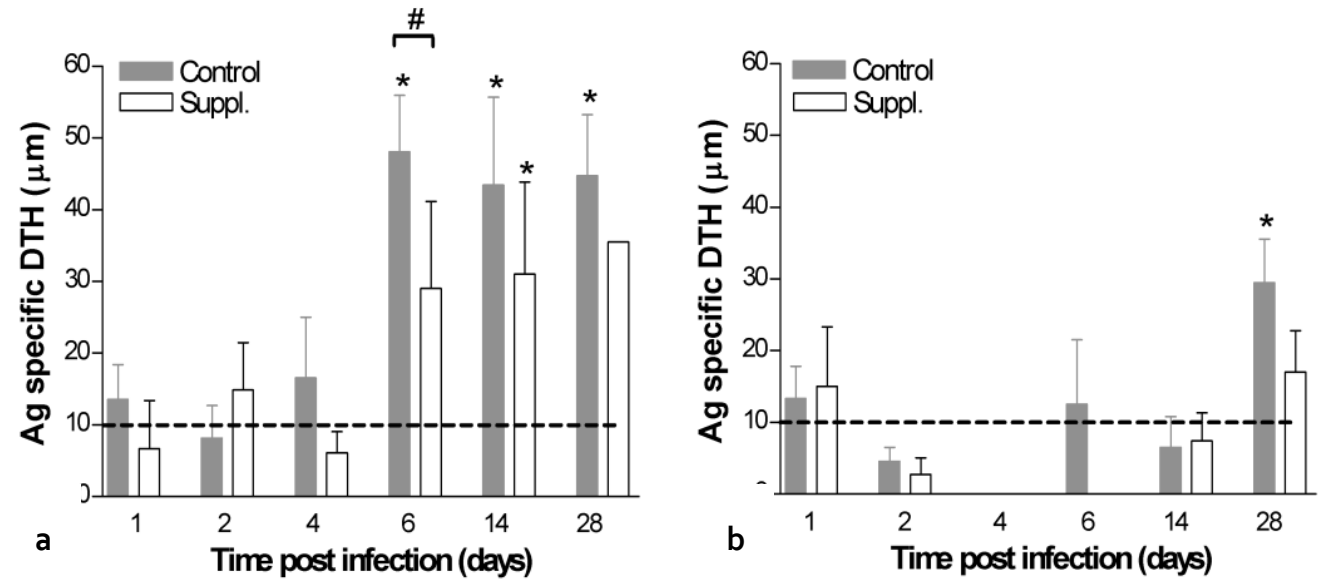

Figure 3. Effect of supplementation on cellular MCMV-specific responses in vivo. $C_{57} \mathrm{BL} / 6 \mathrm{~J}$ mice (a) and $B A L B / c$ mice (b) were divided in groups $(n=30)$ receiving supplemented (black bars) or control (gray bars) diets, starting 2 weeks prior to infection with MCMV and continued until the end of the experiment. All animals $(n=5$ per time point) received an ear challenge with inactivated MCMV, 24 hours before the indicated time points. DTH responses were measured as the ear swelling after 24 hours; i.e. ear thickness measured at challenge was subtracted from ear thickness 24 hours after challenge and corrected for aspecific ear swelling. Data are presented as means per group \pm SEM. $\left(^{*}\right)$ Indicates a significant increase in MCMV specific DTH compared to baseline at $\mathrm{P}<0.05$. (\#) Indicates significant difference between animals receiving supplemented or control diets at indicated time point $\mathrm{P}<0.05$.

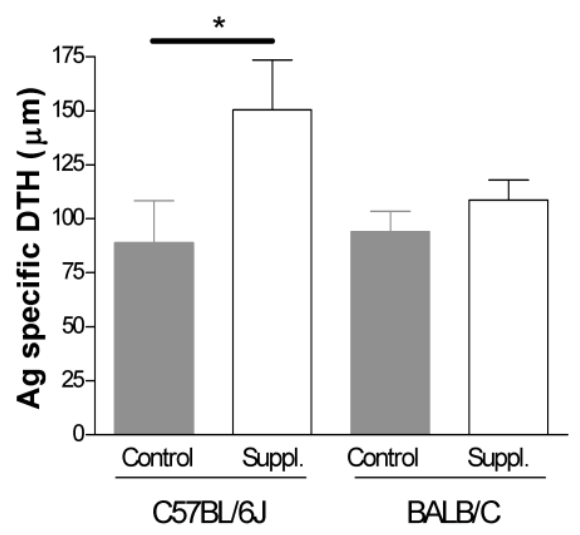

Figure 4. Vaccine-specific DTH responses were analyzed as an in vivo parameter for immune modulation. $\mathrm{C} 57 \mathrm{BL} / 6 \mathrm{~J}$ mice and $\mathrm{BALB} / \mathrm{c}$ mice were divided in groups $(n=5)$ receiving supplemented (black bars) or control (gray bars) diets, starting 2 weeks prior to first vaccination and continued until the end of the experiment. At the end of the experiment (day 28), DTH responses were analyzed by measuring ear swelling 24 hours after antigen injection; i.e. ear thickness measured at challenge was subtracted from ear thickness 24 hours after challenge and corrected for basal ear swelling. Data are presented as means per group \pm SEM. (*) Indicates significant differences between intervention group and the control group at $\mathrm{P}<0.05$. 


\section{Discussion}

The ability of a multi-cellular organism to defend itself against invasion by pathogens (bacteria, fungi, viruses, etc.) depends on its capacity to mount effective immune responses. Dietary supplementation with specific oligosaccharides have been described to modulate various immune functions, suggesting that their application might increase resistance to infection (chapters 3 and 4). The present study shows that dietary supplementation with a specific mixture of oligosaccharides lowered the viral load in several organs at day 1, while it tended to increase levels of anti-viral cytokines in serum. This stimulation of early cytomegalovirus surveillance was observed in MCMVresistant $\mathrm{C} 57 \mathrm{BL} / 6 \mathrm{~J}$ mice but not in the MCMV-sensitive Balb/C mice, suggesting a prominent role for the supplemented oligosaccharides in modulating NK cell responses during cytomegalovirus infection.

Following primary CMV infection of an adult immunocompetent host, virus replication takes place in various tissues. During this early phase, NK cells play an essential role in the innate control of the infection. Early anti-viral responses in MCMV-resistant C57BL/6 mice have been shown to depend largely on NK cell activity (13). In contrast, NK cells in MCMV-sensitive BALB/c mice are not effective in controlling the virus due to the absence of the $\mathrm{Ly} 49 \mathrm{H}$ receptor, leading to a dominance of CD8+ T-cell responses (14). In the present study, we demonstrate that the early immune response can be significantly enhanced in C57BL6, but not in Balb/C mice by dietary supplementation with a specific mixture of oligosaccharides. This suggests that the supplemented diets enhanced the NK cell number and/or activity. In correspondence, other types of oligosaccharides have been reported in literature to stimulate NK cell cytotoxicity (15, 16). Based on these data, several mechanisms are feasible by which oligosaccharides may affect NK cell function. The presently tested mixture of oligosaccharides has been shown to increase the proportions of bifidobacteria and lactobacilli in the large intestine (chapter 4), as well as the levels of fecal short-chain fatty acids (SCFA; chapter 3). Immune-modulatory properties, including the augmentation of NK cell activity, have been attributed to SCFAs (17) and probiotic strains of bifidobacteria and lactobacilli (18, 19). Similar effects may have contributed to the enhanced early MCMV surveillance observed in supplemented mice. Although the details of bacterially-induced immune modulation are not clear, it is possible that bacterial signaling through Toll-like receptor 2 (TLR2) might play a role, as TLR2 has been implicated to play an important role in NK-dependent responses to MCMV (20). The increased serum levels of IFN $\alpha$ and INF $\gamma$ in supplemented C57BL/6J mice may suggest that the mixture of scGOS, lcFOS and pAOS affected dendritic cell (DC) function, thereby leading to increased NK 
cell responses. Plasmacytoid dendritic cells ( $\mathrm{pDC}$ ) are known to produce large quantities of IFN- $\alpha$ and $-\beta$ upon viral challenge, leading to activated NK cells that mediate elevated cytotoxicity. In addition, pDCs have been shown to produce IL-12, which is required for a strong NK-dependent IFN- $\gamma$ response $(21,22)$. Other cell types may be involved as well: a probiotic Lactobacillus strain was reported to augment NK activity by increasing monocyte IL-12 production $(23,24)$. In addition, NKT cells have been shown to increase NK activity, reduce MCMV replication in visceral organs and may be modulated by prebiotic agents (25-28). The carbohydrate structures of oligosaccharides may also interact directly with cells of the immune system. Activation of a $\beta$-glucan receptor by carbohydrates has been shown to prime NK cells and enhance their cytotoxicity towards K562 cells $(29,30)$. Therefore, oligosaccharides that are capable of $\beta$-glucan receptor activation may override the different immune evasive effects of various MCMV genes (31-33). Nonetheless, further investigations are required to establish what mechanisms actually play a role in the observed enhancement of early, probably NK cell-mediated, antiviral response towards MCMV in treated C57BL/6J mice.

Apart from pDCs and NK cells, CD4+ T-cells also contribute to the early antiviral response in the infected host. Early in infection, CMV specific T-cell have the appearance of IFN- $\gamma$-producing Th1 cells, whereas this population is replaced by CD4+CD28- cytotoxic T-cells during latency (34). In view of the intimate relationship between the innate immune responses and adaptive cellular immunity to cytomegalovirus, we decided to investigate the effects of oligosaccharide supplementation on cellular immunity. It was shown previously that immunemodulating properties of specific oligosaccharide mixtures could be detected in vivo by measuring DTH responses (chapter 4). These results were reproduced in the present study; increased influenza-specific DTH responses were shown in supplemented C57BL/6J mice, indicating improved cellular immune responses to the vaccine antigens. Similarly, this technique was applied to study immune responses towards cytomegalovirus antigens. Significant DTH responses were noted in MCMV-infected C57BL/6J mice starting at 6 days p.i, which is in accordance with previous data by Lawson and colleagues (11). Nevertheless, apart from a small though significant difference found at 6 days p.i., the responses were comparable in control and supplemented mice, suggesting that the supplementation did not induce major modulation of the cellular immune response to the virus. In BALB/c mice, DTH responses were almost absent, irrespective of the diet. The difference in the results between $\mathrm{C} 57 \mathrm{BL} / 6 \mathrm{~J}$ and $\mathrm{BALB} / \mathrm{C}$ mice is speculated to be related to strain-related differences in immunological phenotypes. C57BL/6 have been described to be more 
prone to Th1-related immune responses, while BALB/c mice are more prone to Th2related responses (35-37). This is supported by the finding that supplementation did not affect vaccine-specific DTH responses in BALB/c mice either.

The absence of a significantly improved cellular immune response may explain the lack of effect of the oligosaccharide mixture at later time points p.i. on the number of MCMV DNA copies. Although the dose of virus that was used (2.5-5x103 PFU) was rather limited, it might have been sufficient to overrule the early beneficial effects of the prebiotic mixture at later time points p.i. This suggests that the applied prebiotic mixture might be of limited use during acute CMV infections. Nevertheless, supplementation might be useful to raise the threshold of a successful viral infection, or to counteract periodical viral reactivation. It has been demonstrated that $\mathrm{CMV}$ reactivates from latency during periods of severe stress or with increasing age in otherwise healthy persons (38-41) and these periods of subclinical reactivation might be responsible for the suggested long term adverse effects of the virus on e.g. atherosclerosis. However, further studies are needed to investigate whether prebiotics, possibly in combination with probiotic treatment, are able to prevent viral reactivation. In conclusion, this exploratory study describes a model showing nutritional influences on a systemic murine CMV infection. These data warrants further investigation into mechanisms of action of these specific oligosaccharides.

\section{References}

1. Hosono, A., A. Ozawa, R. Kato, Y. Ohnishi, Y. Nakanishi, T. Kimura, and R. Nakamura, Dietary fructooligosaccharides induce immunoregulation of intestinal IgA secretion by murine Peyer's patch cells. Biosci Biotechnol Biochem, 2003. 67(4): p. 758-64.

2. Manhart, N., A. Spittler, H. Bergmeister, M. Mittlbock, and E. Roth, Influence of fructooligosaccharides on Peyer's patch lymphocyte numbers in healthy and endotoxemic mice. Nutrition, 2003. 19(7-8): p. 657-6o.

3. Nakamura, Y., S. Nosaka, M. Suzuki, S. Nagafuchi, T. Takahashi, T. Yajima, N. Takenouchi-Ohkubo, T. Iwase, and I. Moro, Dietary fructooligosaccharides up-regulate immunoglobulin A response and polymeric immunoglobulin receptor expression in intestines of infant mice. Clin Exp Immunol, 2004. 137(1): p. 52-8.

4. Roller, M., G. Rechkemmer, and B. Watzl, Prebiotic inulin enriched with oligofructose in combination with the probiotics Lactobacillus rhamnosus and Bifidobacterium lactis modulates intestinal immune functions in rats. J Nutr, 2004. 134(1): p. 153-6.

5. Buddington, K.K., J.B. Donahoo, and R.K. Buddington, Dietary oligofructose and inulin protect mice from enteric and systemic pathogens and tumor inducers. J Nutr, 2002. 132(3): p. 472-7.

6. Krmpotic, A., I. Bubic, B. Polic, P. Lucin, and S. Jonjic, Pathogenesis of murine cytomegalovirus infection. Microbes Infect, 2003. 5(13): p. 1263-1277. 
7. Arase, H., E.S. Mocarski, A.E. Campbell, A.B. Hill, and L.L. Lanier, Direct recognition of cytomegalovirus by activating and inhibitory NK cell receptors. Science, 2002. 296(5571): p. 1323-6.

8. Scalzo, A.A., N.A. Fitzgerald, C.R. Wallace, A.E. Gibbons, Y.C. Smart, R.C. Burton, and G.R. Shellam, The effect of the Cmv-1 resistance gene, which is linked to the natural killer cell gene complex, is mediated by natural killer cells. J Immunol, 1992. 149(2): p. 581-9.

9. Vliegen, I., S. Herngreen, G. Grauls, C. Bruggeman, and F. Stassen, Improved detection and quantification of mouse cytomegalovirus by real-time PCR. Virus Res, 2003. 98(1): p. 17-25.

10. Bruggeman, C.A., H. Meijer, P.H. Dormans, W.M. Debie, G.E. Grauls, and C.P. van Boven, Isolation of a cytomegalovirus-like agent from wild rats. Arch Virol, 1982. 73(3-4): p. 231-41. Write to the Help Desk NCBI | NLM | NIH Department of Health \& Human Services Privacy Statement | Freedom of Information Act | Disclaimer.

11. Lawson, C.M., J.E. Grundy, and G.R. Shellam, Delayed-type hypersensitivity responses to murine cytomegalovirus in genetically resistant and susceptible strains of mice. J Gen Virol, 1987. 68(Pt 9): p. 2379-88.

12. Vos, A.P., L. M'Rabet, B. Stahl, G. Boehm, and J. Garssen, Immune-modulatory effects and potential working mechanisms of orally applied nondigestible carbohydrates. Crit Rev Immunol, 2007. 27(2): p. 97-140.

13. Daniels, K.A., G. Devora, W.C. Lai, C.L. O'Donnell, M. Bennett, and R.M. Welsh, Murine cytomegalovirus is regulated by a discrete subset of natural killer cells reactive with monoclonal antibody to Ly49H. J Exp Med, 2001. 194(1): p. 29-44.

14. Lathbury, L.J., J.E. Allan, G.R. Shellam, and A.A. Scalzo, Effect of host genotype in determining the relative roles of natural killer cells and $T$ cells in mediating protection against murine cytomegalovirus infection. J Gen Virol, 1996. 77 ( Pt 10): p. 2605-13.

15. Murosaki, S., K. Muroyama, Y. Yamamoto, T. Liu, and Y. Yoshikai, Nigerooligosaccharides augments natural killer activity of hepatic mononuclear cells in mice. Int Immunopharmacol, 2002. 2(1): p. 1519.

16. Yuan, H., J. Song, X. Li, N. Li, and J. Dai, Immunomodulation and antitumor activity of kappacarrageenan oligosaccharides. Cancer Lett, 2006. 243(2): p. 228-34.

17. Pratt, V.C., K.A. Tappenden, M.I. McBurney, and C.J. Field, Short-chain fatty acid-supplemented total parenteral nutrition improves nonspecific immunity after intestinal resection in rats. JPEN J Parenter Enteral Nutr, 1996. 20(4): p. 264-71.

18. Gill, H.S., K.J. Rutherfurd, M.L. Cross, and P.K. Gopal, Enhancement of immunity in the elderly by dietary supplementation with the probiotic Bifidobacterium lactis HNo19. Am J Clin Nutr, 2001. 74(6): p. 833-9.

19. Takeda, K. and K. Okumura, Effects of a fermented milk drink containing Lactobacillus casei strain Shirota on the human NK-cell activity. J Nutr, 2007. 137(3 Suppl 2): p. 791S-3S.

20. Szomolanyi-Tsuda, E., X. Liang, R.M. Welsh, E.A. Kurt-Jones, and R.W. Finberg, Role for TLR2 in NK cell-mediated control of murine cytomegalovirus in vivo. J Virol, 2006. 8o(9): p. 4286-91.

21. Orange, J.S., B. Wang, C. Terhorst, and C.A. Biron, Requirement for natural killer cell-produced interferon gamma in defense against murine cytomegalovirus infection and enhancement of this defense pathway by interleukin 12 administration. J Exp Med, 1995. 182(4): p. 1045-56.

22. Orange, J.S. and C.A. Biron, An absolute and restricted requirement for IL-12 in natural killer cell IFNgamma production and antiviral defense. Studies of natural killer and T cell responses in contrasting viral infections. J Immunol, 1996. 156(3): p. 1138-42.

23. Takeda, K., T. Suzuki, S.I. Shimada, K. Shida, M. Nanno, and K. Okumura, Interleukin-12 is involved in the enhancement of human natural killer cell activity by Lactobacillus casei Shirota. Clin Exp Immunol, 2006. 146(1): p. 109-15. 
24. Shida, K., T. Suzuki, J. Kiyoshima-Shibata, S. Shimada, and M. Nanno, Essential roles of monocytes in stimulating human peripheral blood mononuclear cells with Lactobacillus casei to produce cytokines and augment natural killer cell activity. Clin Vaccine Immunol, 2006. 13(9): p. 997-1003.

25. Mizubuchi, H., T. Yajima, N. Aoi, T. Tomita, and Y. Yoshikai, Isomalto-oligosaccharides polarize Th1like responses in intestinal and systemic immunity in mice. J Nutr, 2005. 135(12): p. 2857-61.

26. Wesley, J. and L. Brossay, NK T cell-NK cell cross-talk: reciprocal interaction and activation? Arch Immunol Ther Exp (Warsz), 2003. 51(2): p. 121-6.

27. van Dommelen, S.L., H.A. Tabarias, M.J. Smyth, and M.A. Degli-Esposti, Activation of natural killer (NK) T cells during murine cytomegalovirus infection enhances the antiviral response mediated by NK cells. J Virol, 2003. 77(3): p. 1877-84.

28. Ortaldo, J.R., H.A. Young, R.T. Winkler-Pickett, E.W. Bere, Jr., W.J. Murphy, and R.H. Wiltrout, Dissociation of NKT stimulation, cytokine induction, and NK activation in vivo by the use of distinct TCR-binding ceramides. J Immunol, 2004. 172(2): p. 943-53.

29. Di Renzo, L., E. Yefenof, and E. Klein, The function of human NK cells is enhanced by beta-glucan, a ligand of $\mathrm{CR}_{3}$ (CD11b/CD18). Eur J Immunol, 1991. 21(7): p. 1755-8.

30. Vetvicka, V., B.P. Thornton, and G.D. Ross, Soluble beta-glucan polysaccharide binding to the lectin site of neutrophil or natural killer cell complement receptor type $3\left(C_{11} b_{/} C_{1} 8\right)$ generates a primed state of the receptor capable of mediating cytotoxicity of $\mathrm{iC}_{3} \mathrm{~b}$-opsonized target cells. J Clin Invest, 1996. 98(1): p. 50-61.

31. Krmpotic, A., D.H. Busch, I. Bubic, F. Gebhardt, H. Hengel, M. Hasan, A.A. Scalzo, U.H. Koszinowski, and S. Jonjic, MCMV glycoprotein gp40 confers virus resistance to CD8+ T cells and NK cells in vivo. Nat Immunol, 2002. 3(6): p. 529-35.

32. Krmpotic, A., M. Hasan, A. Loewendorf, T. Saulig, A. Halenius, T. Lenac, B. Polic, I. Bubic, A. Kriegeskorte, E. Pernjak-Pugel, M. Messerle, H. Hengel, D.H. Busch, U.H. Koszinowski, and S. Jonjic, NK cell activation through the NKG2D ligand MULT-1 is selectively prevented by the glycoprotein encoded by mouse cytomegalovirus gene m145. J Exp Med, 2005. 201(2): p. 211-20.

33. Hasan, M., A. Krmpotic, Z. Ruzsics, I. Bubic, T. Lenac, A. Halenius, A. Loewendorf, M. Messerle, H. Hengel, S. Jonjic, and U.H. Koszinowski, Selective down-regulation of the NKG2D ligand H6o by mouse cytomegalovirus m155 glycoprotein. J Virol, 2005. 79(5): p. 2920-30.

34. van Leeuwen, E.M., E.B. Remmerswaal, M.H. Heemskerk, I.J. ten Berge, and R.A. van Lier, Strong selection of virus-specific cytotoxic $\mathrm{CD}_{4}+\mathrm{T}$-cell clones during primary human cytomegalovirus infection. Blood, 2006. 108(9): p. 3121-7.

35. Lawson, C.M., J.E. Grundy, and G.R. Shellam, Delayed-type hypersensitivity responses to murine cytomegalovirus in genetically resistant and susceptible strains of mice. J Gen Virol, 1987. 68 ( Pt 9): p. $2379-88$.

36. Herz, U., A. Braun, R. Ruckert, and H. Renz, Various immunological phenotypes are associated with increased airway responsiveness. Clin Exp Allergy, 1998. 28(5): p. 625-34.

37. Sacks, D. and N. Noben-Trauth, The immunology of susceptibility and resistance to Leishmania major in mice. Nat Rev Immunol, 2002. 2(11): p. 845-58.

38. Zhang, Y., W.J. Lamm, R.K. Albert, E.Y. Chi, W.R. Henderson, Jr., and D.B. Lewis, Influence of the route of allergen administration and genetic background on the murine allergic pulmonary response. Am J Respir Crit Care Med, 1997. 155(2): p. 661-9.

39. Glaser, R., J.K. Kiecolt-Glaser, C.E. Speicher, and J.E. Holliday, Stress, loneliness, and changes in herpesvirus latency. J Behav Med, 1985. 8(3): p. 249-60.

40. Ling, P.D., J.A. Lednicky, W.A. Keitel, D.G. Poston, Z.S. White, R. Peng, Z. Liu, S.K. Mehta, D.L. Pierson, C.M. Rooney, R.A. Vilchez, E.O. Smith, and J.S. Butel, The dynamics of herpesvirus and polyomavirus reactivation and shedding in healthy adults: a 14-month longitudinal study. J Infect Dis, 2003. 187(10): p. 1571-80. 
41. Mehta, S.K., R.P. Stowe, A.H. Feiveson, S.K. Tyring, and D.L. Pierson, Reactivation and shedding of cytomegalovirus in astronauts during spaceflight. J Infect Dis, 2000. 182(6): p. 1761-4.

42. McVoy, M.A. and S.P. Adler, Immunologic evidence for frequent age-related cytomegalovirus reactivation in seropositive immunocompetent individuals. J Infect Dis, 1989. 160(1): p. 1-10. 


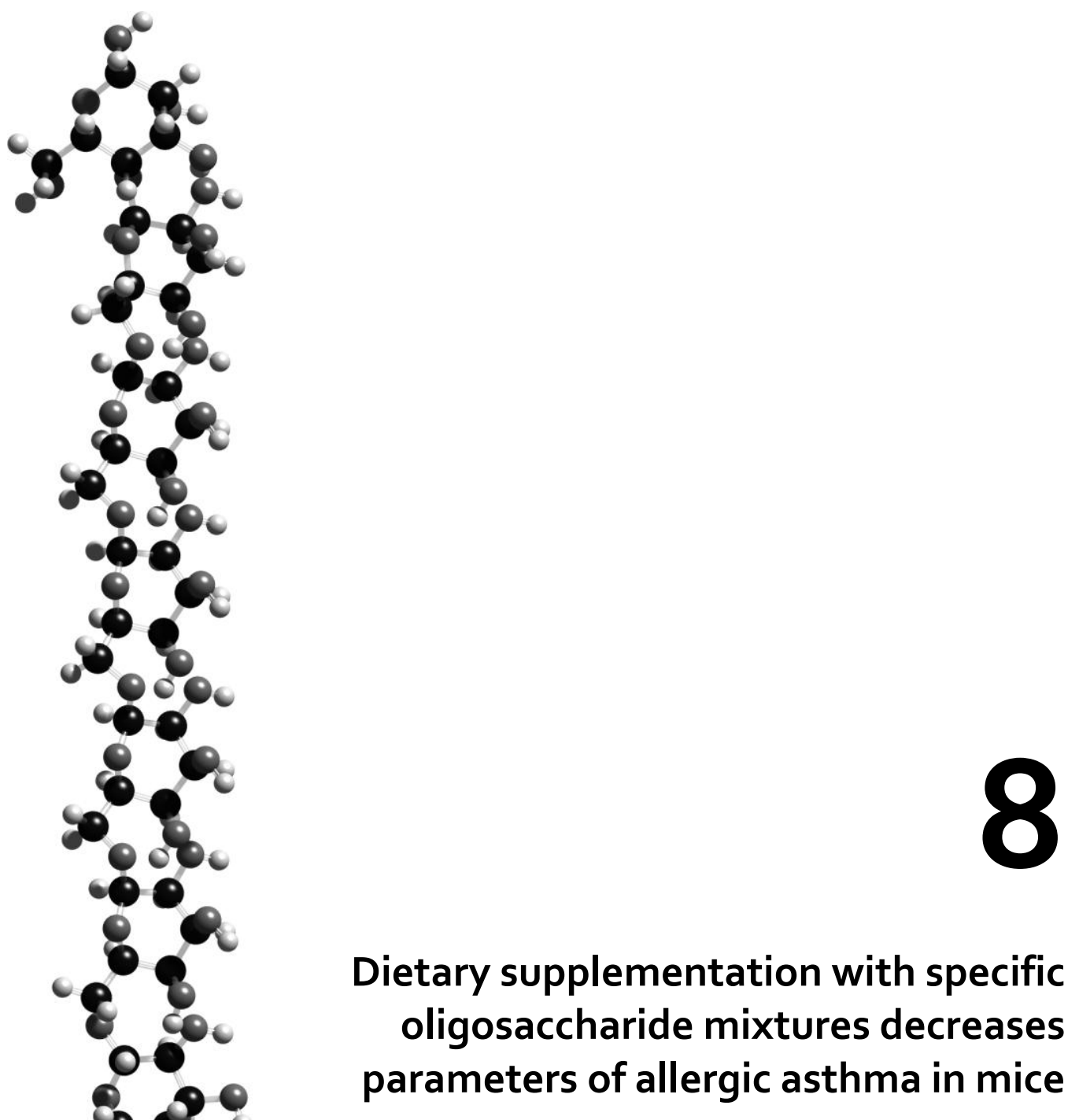

A.P. Vos

B.C. van Esch

B. Stahl

L. M'Rabet

G. Folkerts

F.P. Nijkamp

J. Garssen

Int Immunopharmacol 2007, 7(12):1582-7 


\section{Abstract}

Specific mixtures of prebiotic oligosaccharides showed immune-modulatory effects in previous murine vaccination experiments, suggesting a shift towards T-helper 1 (Th1) immunity. These mixtures consisted of galactooligosaccharides (scGOS) and long-chain fructooligosaccharides (lcFOS) in a 9:1 ratio (Immunofortis), with or without pectinderived acidic oligosaccharides (pAOS). To investigate whether these mixtures could suppress Th2-related responses, they were tested in an ovalbumin (OVA)-induced model for experimental allergic asthma in Balb/c mice. Supplementation with two mixtures of scGOS/lcFOS and scGOS/lcFOS/pAOS at approximately $1 \%$ (w/w $\%$ net oligosaccharides) in the diet, starting two weeks before OVA sensitization and lasting until the end of the experiment, led to a reduction in several parameters of allergic asthma. The OVA-induced airway inflammation and hyperresponsiveness was significantly suppressed by both mixtures. Moreover, OVA-specific IgE titers were decreased by more than $25 \%$, although this effect was not significant. The effects of the oligosaccharide mixture with pAOS appeared to be more pronounced than the effects of the scGOS/lcFOS mixture without pAOS, but a direct comparison between the mixtures was not made. Overall, the results further support the hypothesis that the specific mixtures of oligosaccharides modulate the Th1/Th2 balance by enhancing Th1related and suppressing Th2-related parameters.

\section{Introduction}

Dietary oligosaccharides have been shown to modulate immune function in various recent reports. In the intestine, beneficial effects have been shown on parameters of inflammation (1-3) and modulatory effects have been shown on cell populations in Peyer's patches (PP) (4-6). A few reports on systemic immune modulatory effects have been published as well $(7,8)$.

We have previously reported systemic immune modulatory effects after oral supplementation with specific mixtures of oligosaccharides, designed to mimic the prebiotic and immunological functions of breast milk oligosaccharides as closely as possible. These mixtures consisted of galactooligosaccharides (scGOS) and lcFOS in a 9:1 ratio (Immunofortis), with or without pectin-derived acidic oligosaccharides (pAOS) that consist of partly unsaturated and partly methylated galacturonic acids (9, 
10). Enhanced T-helper 1 (Th1) dependent delayed-type hypersensitivity responses after vaccination were shown repeatedly in an influenza vaccination model in C57BL/6 mice (chapters 3 and 4). Additionally, inhibition of Th2 cytokine production was observed in pAOS supplemented animals (chapter 4), leading us to hypothesize that the dietary interventions induced a mild shift in the Th1/Th2 balance towards Th1 responses in healthy $\mathrm{C} 57 \mathrm{BL} / 6$ mice.

To verify this hypothesis in a different animal model, the specific oligosaccharide mixtures were tested in a model for experimental allergic asthma in Th2-prone Balb/c mice. Since Th1 and Th2 responses are thought to be reciprocally regulated, it was hypothesized that dietary intervention with the specific oligosaccharide mixtures could decrease parameters of allergic asthma in this Th2-related model $(11,12)$. This hypothesis was tested in two separate experiments.

\section{Materials and methods}

\section{Animals and diets}

Male BALB/cByJIco SPF mice, 5-8 weeks of age with 20-25 g of weight, were obtained from Charles River (Someren, The Netherlands). The mice were accustomed to their new environment for at least 1 week before the start of the experiment. All experiments were approved by an independent animal experiments committee.

All animals received semi-purified AIN-93G-based diets (Research Diet Services, Wijk bij Duurstede, The Netherlands). All supplemented oligosaccharide products were exchanged for the same amount of total carbohydrates, mixed into the AIN-93G diet and pressed into pellets.

The active ingredients in the diets were a mixture of scGOS (Vivinal GOS, Borculo Domo, Zwolle, The Netherlands;) and lcFOS (Raftiline HP, Orafti, Wijchen, The Netherlands;) in a 9:1 ratio (Immunofortis). Additionally, pectin-derived pAOS were used (kindly provided by Südzucker AG, Mannheim, Germany). Two supplemented test diets were compared with AIN93G control diet in two separate experiments. The diets were described in detail and were shown to induce immune modulatory effects previously (chapter 4 ). In short, one test diet contained a net amount of $1 \%(\mathrm{w} / \mathrm{w})$ scGOS/lcFOS in a 9:1 ratio. The other test diet contained approximately $1 \%(\mathrm{w} / \mathrm{w})$ net oligosaccharides, consisting of $83 \%$ scGOS/lcFOS $+17 \%$ pAOS. All mice were given control AIN-93G diets upon arrival at the animal facility for a minimum of 7 days. The dietary interventions in supplementation groups started 14 days before the primary sensitization and lasted during the entire experiment. 


\section{OVA sensitization and aerosol challenge}

Mice were sensitized to ovalbumin by two intraperitoneal injections with $10 \mu \mathrm{g}$ ovalbumin grade V (Sigma Aldrich, Zwijndrecht, The Netherlands) in $2.25 \mathrm{mg} / 0.1 \mathrm{ml}$ AlumInject (Pierce, Perbio Science Nederland, Etten-Leur, The Netherlands) in PBS on day 0 and 7. Sham-sensitized animals received $0.1 \mathrm{ml}$ PBS/AlumInject only. Each experiment consisted of four groups; the control and supplemented diets were tested both in sham and OVA-sensitized mice. All groups consisted of 9 animals.

OVA sensitized and sham sensitized mice were exposed to $10 \mathrm{mg} / \mathrm{ml}$ nebulized OVA aerosols in PBS using Pari LC Star (Pari IS-2, Pari-Werk GmbH, Starnberg, Germany). For each exposure, the animals were placed in an aerosol cabin for 20 minutes. Animals were aerosol challenged on day 35, 38 and 41 after the first sensitization.

\section{Airway hyperresponsiveness}

Airway responsiveness was measured in vivo 24 hours after the last aerosol exposure in conscious unrestrained mice using a plethysmograph (Buxco, EMKA Technologies, France) as described by Hanemann et al. (13). The mice were exposed for 3 minutes to doubling doses of aerosolized methacholine (Mch) ranging from $1.56 \mathrm{mg} / \mathrm{ml}$ to 50 $\mathrm{mg} / \mathrm{ml}$; after exposure to a methacholine dose lung function was measured for 3 minutes. From the lung function parameters peak expiratory flow (PEF), tidal volume (TV), expiratory time (Te) and frequency ( $\mathrm{f}$ ) the enhanced pause (PENH) parameter can be calculated with the formula ((Te/RT)-1).(PEF/PIF). It has previously been demonstrated that PENH correlates strongly to airway resistance in this allergic asthma model in Balb/c mice (13-15).

\section{Broncho-alveolar lavage}

Broncho-alveolar lavage (BAL) was performed in the same animals that were used for in vivo airway hyperresponsiveness measurements. In pilot experiments it was found that combining these techniques had no effect on the total number of lavage cells. Mice received a lethal dose of pentobarbital sodium $(300 \mathrm{mg} / \mathrm{kg}$ body weight intraperitoneally). The lungs were filled with $1 \mathrm{ml}$ aliquots of pyrogen-free saline $(0.9 \%$ $\mathrm{NaCl})$ supplemented with protease inhibitor cocktail tablet (1 tablet/ $100 \mathrm{ml})$ and bovine serum albumin $(5 \mathrm{~g} / 100 \mathrm{ml})$ of $37^{\circ} \mathrm{C}$ in situ. Fluid was collected in a plastic tube on ice $\left(4^{\circ} \mathrm{C}\right.$ ) (totally $1 \mathrm{ml}$ ). This procedure was repeated 3 times with aliquots of pyrogen free saline $(0.9 \% \mathrm{NaCl})$. The BAL cells were centrifuged $\left(400 \mathrm{~g}, 4^{\circ} \mathrm{C}, 5 \mathrm{~min}\right)$, all pellets from one animal were pooled, resuspended and counted by use of a BürkerTürk chamber (magnification 100x). 


\section{Measurement of OVA-specific IgE in serum}

Flat-bottom 96-well plates (Nunc Maxisorp, Life Technologies, Breda, The Netherlands) were coated overnight at $4{ }^{\circ} \mathrm{C}$ with capture antibody $(1 \mu \mathrm{g} / \mathrm{ml}$ purified $\mathrm{Rt} \times \mathrm{Ms}$ IgE; Pharmingen, San Diego, CA, USA) in sodium carbonate buffer ( $\mathrm{pH}$ 9.4). After coating and in between subsequent incubation steps, plates were washed with PBS containing 0.05\% Tween-20. Plates were blocked (2 mM EDTA, $136.9 \mathrm{mM} \mathrm{NaCl}, 50 \mathrm{mM}$ Tris, 0.5\% BSA and $0.05 \%$ Tween-20, pH 7.2, 1 hour, room temperature (RT)). Samples and standards (serum pool from OVA immunized mice, CIP, Utrecht, NL) were applied (100 $\mu \mathrm{l} /$ well, 2 hours, RT), followed by OVA coupled to Digoxigenin (DIG; Roche Diagnostics, Mannheim, Germany; $100 \mu \mathrm{l} /$ well, 1 hour, RT) and anti-DIG-peroxidase (Roche Diagnostics; 1 hour, RT). After the final washing, $0.4 \mathrm{mg} / \mathrm{ml}$ ophenylenediamine-dihydrochloride in PBS containing $0.04 \%$ hydrogen peroxide was added. The reaction was stopped after approx. 5 minutes using $4 \mathrm{M} \mathrm{H}_{2} \mathrm{SO}_{4}$. Optical density was measured at $492 \mathrm{~nm}$.

\section{Analysis and statistical calculations}

Data were analyzed using Graphpad Prism 4.03 software and expressed as arithmetic average \pm standard error of the mean (SEM). ANOVA and post-hoc Dunnett's test was performed to compare the airway hyperresponsiveness data from multiple groups with one control group. For direct comparisons between the sensitized control and sensitized supplemented groups, the one-sided Student's T-test (PENH and BAL inflammatory cells) or Mann-Whitney U-test (IgE titers) was used to evaluate whether supplementation reduced parameters of allergic asthma. A $p$ value of $p<0.05$ was considered significant.

\section{Results}

\section{Airway hyperresponsiveness}

The airway responsiveness to increasing doses of Mch, as measured by PENH, was similar for unsensitized mice in both experiments, regardless of the diet. Airway hyperresponsiveness was observed in sensitized control animals in both experiments; the PENH was significantly higher compared to unsensitized control animals in five out of six concentrations of Mch and the largest effect occurred at the highest concentrations. Both supplemented diets suppressed the development of the OVAinduced airway hyperresponsiveness in sensitized mice (Figure 1). In scGOS/lcFOS fed 

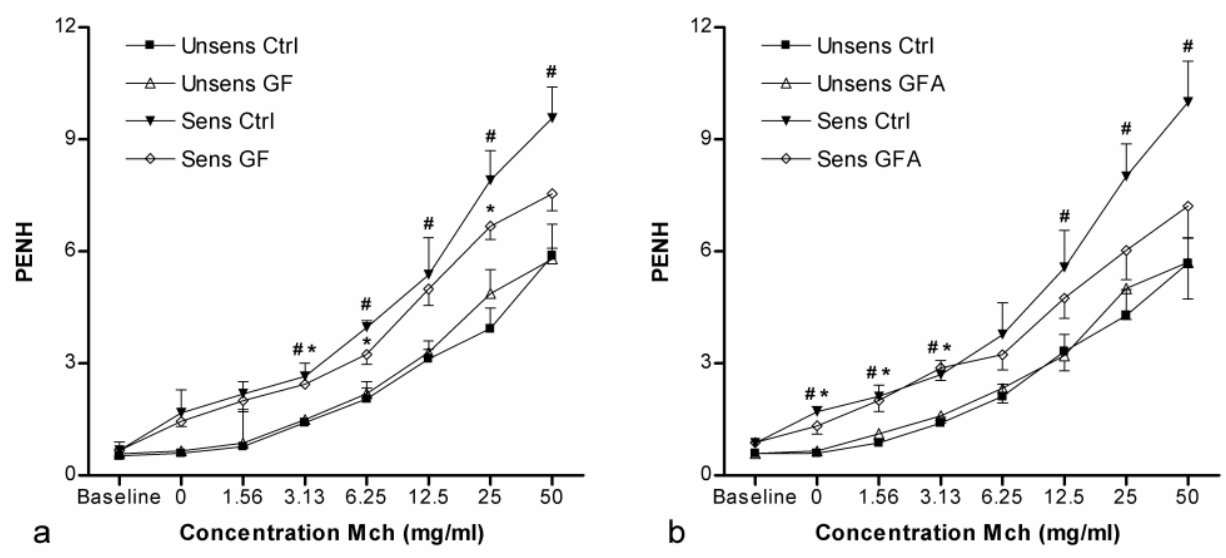

Figure 1. Dietary intervention reduces airway hyperresponsiveness. The effects of dietary intervention with (a) scGOS/lcFOS in a 9:1 ratio (GF) and (b) scGOS/lcFOS/pAOS (GFA) were measured on the PENH parameter, measured in conscious unrestrained ovalbumin sensitized (Sens) and unsensitized mice (Unsens). After a baseline measurement, the mice were challenged with increasing doses of aerosolized Mch in PBS. Values are means \pm SEM ( $n=9$ per group); \# indicates $p<0.05$ unsensitized control mice versus sensitized control mice; * indicates $p<0.05$ unsensitized control mice versus sensitized supplemented mice.

mice, the PENH was not significantly different from unsensitized control mice at 12.5 and $50 \mathrm{mg} / \mathrm{ml} \mathrm{Mch}$, in contrast to sensitized control mice. The same effect was observed in scGOS/lcFOS/pAOS supplemented mice at the three highest concentrations of Mch. Supplementation did not affect the increased responses at lower Mch concentrations, but the maximal responses were significantly decreased by $21 \%(\mathrm{p}=0.02)$ and $27 \%$ $(p=0.03)$ in the $s c G O S / l c F O S$ and scGOS/lcFOS/pAOS diet groups, compared directly to the sensitized control group.

\section{Pulmonary inflammatory cellular response}

The effects of dietary supplementation with oligosaccharides on the number of inflammatory cells in BAL fluid are shown in Figure 2. Low numbers of cells were observed in unsensitized animals irrespective of diet (range 19.2 - 33.9 x103). OVA challenge dramatically increased BAL cellularity in the sensitized groups. Although airway inflammation was still present in both supplemented groups of sensitized mice, the number of cells in the BAL were significantly decreased compared to the control diets (control vs. scGOS/lcFOS: $336.8 \pm 34.5$ vs. $244.9 \pm 9.8 \times 10^{3}, \mathrm{p}=0.01$; control vs. scGOS/lcFOS/pAOS: $353.9 \pm 34.9$ vs. $156.6 \pm 25.1 \times 10^{3}, \mathrm{p}<0.001$ ). The inflammatory cells were not differentiated in these experiments, but have been shown to consist of a large majority of eosinophils in previous comparable experiments (16). 

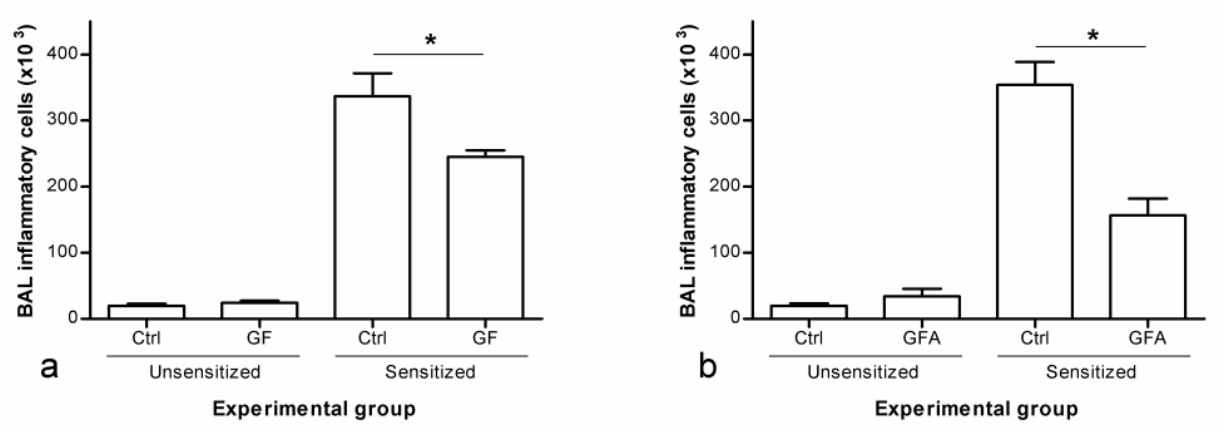

Figure 2. Dietary intervention reduces the number of inflammatory cells in BAL fluid. The effects dietary intervention with (a) scGOS/lcFOS in a 9:1 ratio (GF) and (b) scGOS/lcFOS/pAOS (GFA) are shown on the total number of inflammatory cells in BAL fluid. Values are means \pm SEM ( $n=9$ per group); * indicates $p<0.05$ sensitized control mice versus sensitized supplemented mice.

\section{IgE serum levels}

OVA-specific IgE levels were expressed as arbitrary units (AU), as shown in Figure 3. Background levels were measured in unsensitized mice fed control and supplemented diets (range: 2.4 - $4.6 \mathrm{AU}$ ), but high titers were measured in all sensitized animals. The average IgE titers in mice that were fed scGOS/lcFOS and scGOS/lcFOS/pAOS containing diets were $28 \%$ and $38 \%$ lower than in control diet fed animals, but the differences were not significant (control vs. scGOS/lcFOS: $89.2 \pm 17.7$ vs. $64.7 \pm 4.4 \mathrm{AU}$, $\mathrm{p}=0.13$; control vs. scGOS/lcFOS/pAOS: $78.6 \pm 17.8$ vs. $48.5 \pm 6.7 \mathrm{AU}, \mathrm{p}=0.17$ ).
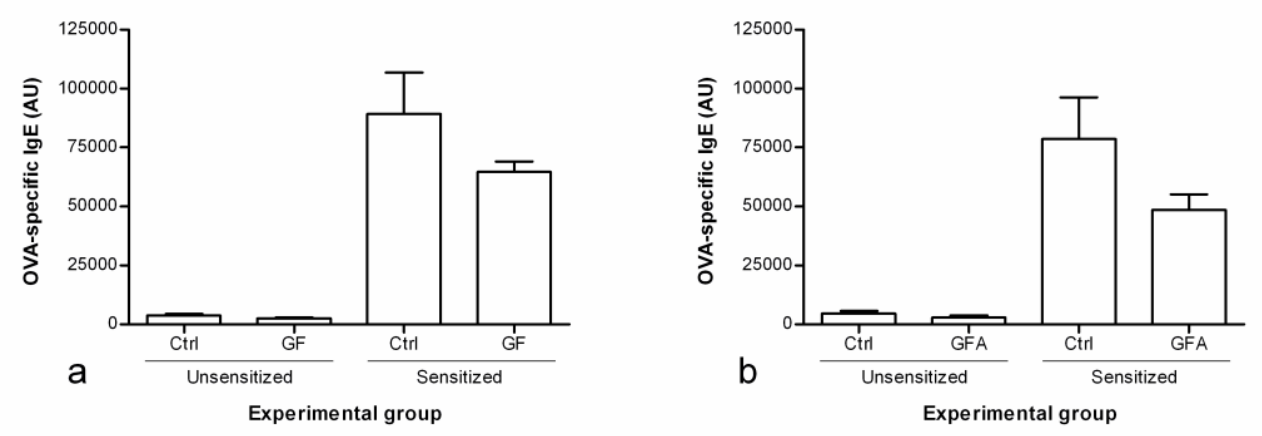

Figure 3. The effect of dietary intervention on OVA-specific IgE titers in serum. The effects dietary intervention with (a) scGOS//cFOS in a 9:1 ratio (GF) and (b) scGOS/lcFOS/pAOS (GFA) on the OVA-specific IgE titers in serum, measured by ELISA. No significant differences were observed between sensitized control and sensitized supplemented groups. 


\section{Discussion}

In accordance with the hypothesis, dietary intervention with scGOS/lcFOS in a 9:1 ratio (Immunofortis) and with scGOS/lcFOS/pAOS decreased several parameters of allergic asthma in Balb/c mice. Significant effects were observed on airway hyperreactivity and the number of inflammatory cells in the BAL fluid. Additionally, OVA-specific IgE levels were decreased by more than $25 \%$ in both supplemented groups, although these differences were not significant. The basal airway reactivity in the OVA-sensitized and challenged groups was higher than in the unsensitized groups, suggesting that a methacholine-independent mechanism plays a role but was unaffected by the dietary intervention. It appears that the intervention only suppressed the Mch-dependent hyperresponsiveness, which is most evident at the highest concentrations. Overall, the results show that supplementation with the specific oligosaccharide mixtures decreases the manifestation of experimental allergic asthma in this model.

These results fit well with data from previous murine vaccination experiments, in which enhanced Th1 dependent DTH responses and reduced Th2 cytokine production ex vivo were observed in healthy young adult C57BL/6 mice (chapter 3 and 4 ). The combined data from all experiments are consistent with the notion that the specific mixtures of oligosaccharides induce a shift in the Th1/Th2 balance towards Th1 responses, in healthy or allergic mouse models using different strains. Although the effects shown in this paper were comparable for both test diets, the effects of the scGOS/lcFOS/pAOS containing diet appeared to be more pronounced than the effects of the scGOS/lcFOS diet. This difference corresponds to previous observations in the vaccination model, in which the combination of all three types of oligosaccharides was significantly more effective than scGOS/lcFOS or pAOS alone (chapter 4). However, since both test diets were tested in two separate experiments in the allergic asthma model, no direct comparison could be made in the present study.

Anti-allergic effects of prebiotics have not been described widely in literature. However, Fujitani and coworkers have very recently demonstrated beneficial effects of $5 \%(w / w)$ short-chain FOS supplementation on parameters of OVA-induced food allergy in mice, leading to reduced numbers of mast cells and edema formation in the duodenum (17). Additionally, Sonoyama et al. have shown that 5\% (w/w) $\alpha$-linked GOS and raffinose reduced airway eosinophilia and Th2 cytokine production in OVA sensitized rats (7).

In the current study, supplementation with the specific oligosaccharide mixtures in both test diets proved to be effective at a low dose that is relevant for various human 
applications, including infant formulas. It was previously shown that comparable doses of scGOS/lcFOS induced bifidogenic effects when applied in an infant formula, resulting in a composition of the intestinal microbiota that was closer to that of breast fed infants than of standard formula fed infants (18). It has been described that the incidence of atopic dermatitis (AD) and early childhood wheezing is higher in standard non-hydrolyzed formula fed infants than in breast fed infants (19-22). Similar to the effects on the microbiota, the current results suggest that addition of the specific mixtures of oligosaccharides to infant formula may help to make it closer to breast milk with regard to the development of atopy and wheezing. This notion is supported by the first clinical results that are available: in children that received scGOS/lcFOScontaining formula during the first 6 months of life, several allergic symptoms were reduced significantly, even until these children were two years of age $(23,24)$. Additional clinical studies are currently ongoing.

\section{References}

1.

2.

7.

Welters, C.F., E. Heineman, F.B. Thunnissen, A.E. van den Bogaard, P.B. Soeters, and C.G. Baeten, Effect of dietary inulin supplementation on inflammation of pouch mucosa in patients with an ileal pouch-anal anastomosis. Dis Colon Rectum, 2002. 45(5): p. 621-7.

Cherbut, C., C. Michel, and G. Lecannu, The prebiotic characteristics of fructooligosaccharides are necessary for reduction of TNBS-induced colitis in rats. J Nutr, 2003. 133(1): p. 21-7.

Lara-Villoslada, F., O. de Haro, D. Camuesco, M. Comalada, J. Velasco, A. Zarzuelo, J. Xaus, and J. Galvez, Short-chain fructooligosaccharides, in spite of being fermented in the upper part of the large intestine, have anti-inflammatory activity in the TNBS model of colitis. Eur J Nutr, 2006.

Hosono, A., A. Ozawa, R. Kato, Y. Ohnishi, Y. Nakanishi, T. Kimura, and R. Nakamura, Dietary fructooligosaccharides induce immunoregulation of intestinal IgA secretion by murine Peyer's patch cells. Biosci Biotechnol Biochem, 2003. 67(4): p. 758-64.

Manhart, N., A. Spittler, H. Bergmeister, M. Mittlbock, and E. Roth, Influence of fructooligosaccharides on Peyer's patch lymphocyte numbers in healthy and endotoxemic mice. Nutrition, 2003. 19(7-8): p. 657-6o.

Roller, M., G. Rechkemmer, and B. Watzl, Prebiotic inulin enriched with oligofructose in combination with the probiotics Lactobacillus rhamnosus and Bifidobacterium lactis modulates intestinal immune functions in rats. J Nutr, 2004. 134(1): p. 153-6.

Sonoyama, K., H. Watanabe, J. Watanabe, N. Yamaguchi, A. Yamashita, H. Hashimoto, E. Kishino, K. Fujita, M. Okada, S. Mori, S. Kitahata, and J. Kawabata, Allergic airway eosinophilia is suppressed in ovalbumin-sensitized Brown Norway rats fed raffinose and alpha-linked galactooligosaccharide. J Nutr, 2005. 135(3): p. 538-43.

Buddington, K.K., J.B. Donahoo, and R.K. Buddington, Dietary oligofructose and inulin protect mice from enteric and systemic pathogens and tumor inducers. J Nutr, 2002. 132(3): p. 472-7.

Fanaro, S., J. Jelinek, B. Stahl, G. Boehm, R. Kock, and V. Vigi, Acidic oligosaccharides from pectin hydrolysate as new component for infant formulae: effect on intestinal flora, stool characteristics, and pH. J Pediatr Gastroenterol Nutr, 2005. 41(2): p. 186-90. 
10. Boehm, G., S. Fanaro, J. Jelinek, B. Stahl, and A. Marini, Prebiotic concept for infant nutrition. Acta Paediatr Suppl, 2003. 91(441): p. 64-7.

11. Corry, D.B., H.G. Folkesson, M.L. Warnock, D.J. Erle, M.A. Matthay, J.P. Wiener-Kronish, and R.M. Locksley, Interleukin 4, but not interleukin 5 or eosinophils, is required in a murine model of acute airway hyperreactivity. J Exp Med, 1996. 183(1): p. 109-17.

12. Grunig, G., M. Warnock, A.E. Wakil, R. Venkayya, F. Brombacher, D.M. Rennick, D. Sheppard, M. Mohrs, D.D. Donaldson, R.M. Locksley, and D.B. Corry, Requirement for IL-13 independently of IL-4 in experimental asthma. Science, 1998. 282(5397): p. 2261-3.

13. Hamelmann, E., J. Schwarze, K. Takeda, A. Oshiba, G.L. Larsen, C.G. Irvin, and E.W. Gelfand, Noninvasive measurement of airway responsiveness in allergic mice using barometric plethysmography. Am J Respir Crit Care Med, 1997. 156(3 Pt 1): p. 766-75. Lomask, M., Further exploration of the Penh parameter. Exp Toxicol Pathol, 2006. 57 Suppl 2: p. 1320.

15. Adler, A., G. Cieslewicz, and C.G. Irvin, Unrestrained plethysmography is an unreliable measure of airway responsiveness in BALB/C and $\mathrm{C}_{57} \mathrm{BL} / 6$ mice. J Appl Physiol, 2004. 97(1): p. 286-92.

16. Smit, J.J., H. Van Loveren, M.O. Hoekstra, M.A. Schijf, G. Folkerts, and F.P. Nijkamp, Mycobacterium vaccae administration during allergen sensitization or challenge suppresses asthmatic features. Clin Exp Allergy, 2003. 33(8): p. 1083-9.

17. Fujitani, S., K. Ueno, T. Kamiya, T. Tsukahara, K. Ishihara, T. Kitabayashi, and K. Itabashi, Increased Number of CCR4-positive Cells in the Duodenum of Ovalbumin-induced Food Allergy Model NC/jic Mice and Antiallergic Activity of Fructooligosaccharides. Allergol Int, 2007. 56(2).

18. Knol, J., P. Scholtens, C. Kafka, J. Steenbakkers, S. Gro, K. Helm, M. Klarczyk, H. Schopfer, H.M. Bockler, and J. Wells, Colon microflora in infants fed formula with galacto- and fructooligosaccharides: more like breast-fed infants. J Pediatr Gastroenterol Nutr, 2005. 40(1): p. 36-42.

19. Friedman, N.J. and R.S. Zeiger, The role of breast-feeding in the development of allergies and asthma. J Allergy Clin Immunol, 2005. 115(6): p. 1238-48.

20. Gdalevich, M., D. Mimouni, M. David, and M. Mimouni, Breast-feeding and the onset of atopic dermatitis in childhood: a systematic review and meta-analysis of prospective studies. J Am Acad Dermatol, 2001. 45(4): p. 520-7.

21.

Gdalevich, M., D. Mimouni, and M. Mimouni, Breast-feeding and the risk of bronchial asthma in childhood: a systematic review with meta-analysis of prospective studies. J Pediatr, 2001. 139(2): p. 261-6.

22. van Odijk, J., I. Kull, M.P. Borres, P. Brandtzaeg, U. Edberg, L.A. Hanson, A. Host, M. Kuitunen, S.F. Olsen, S. Skerfving, J. Sundell, and S. Wille, Breastfeeding and allergic disease: a multidisciplinary review of the literature (1966-2001) on the mode of early feeding in infancy and its impact on later atopic manifestations. Allergy, 2003. 58(9): p. 833-43.

23. Arslanoglu, S., G. Moro, J. Schmitt, and G. Boehm, Early dietary intervention with a mixture of prebiotic oligosaccharides reduces the incidence of allergy associated symptoms and infections during the first 2 years of life. J Pediatr Gastroenterol Nutr, 2007. 44(S1): p. e217.

24. Moro, G., S. Arslanoglu, B. Stahl, J. Jelinek, U. Wahn, and G. Boehm, A mixture of prebiotic oligosaccharides reduces the incidence of atopic dermatitis during the first six months of age. Arch Dis Child, 2006. 91(10): p. 814-9. 


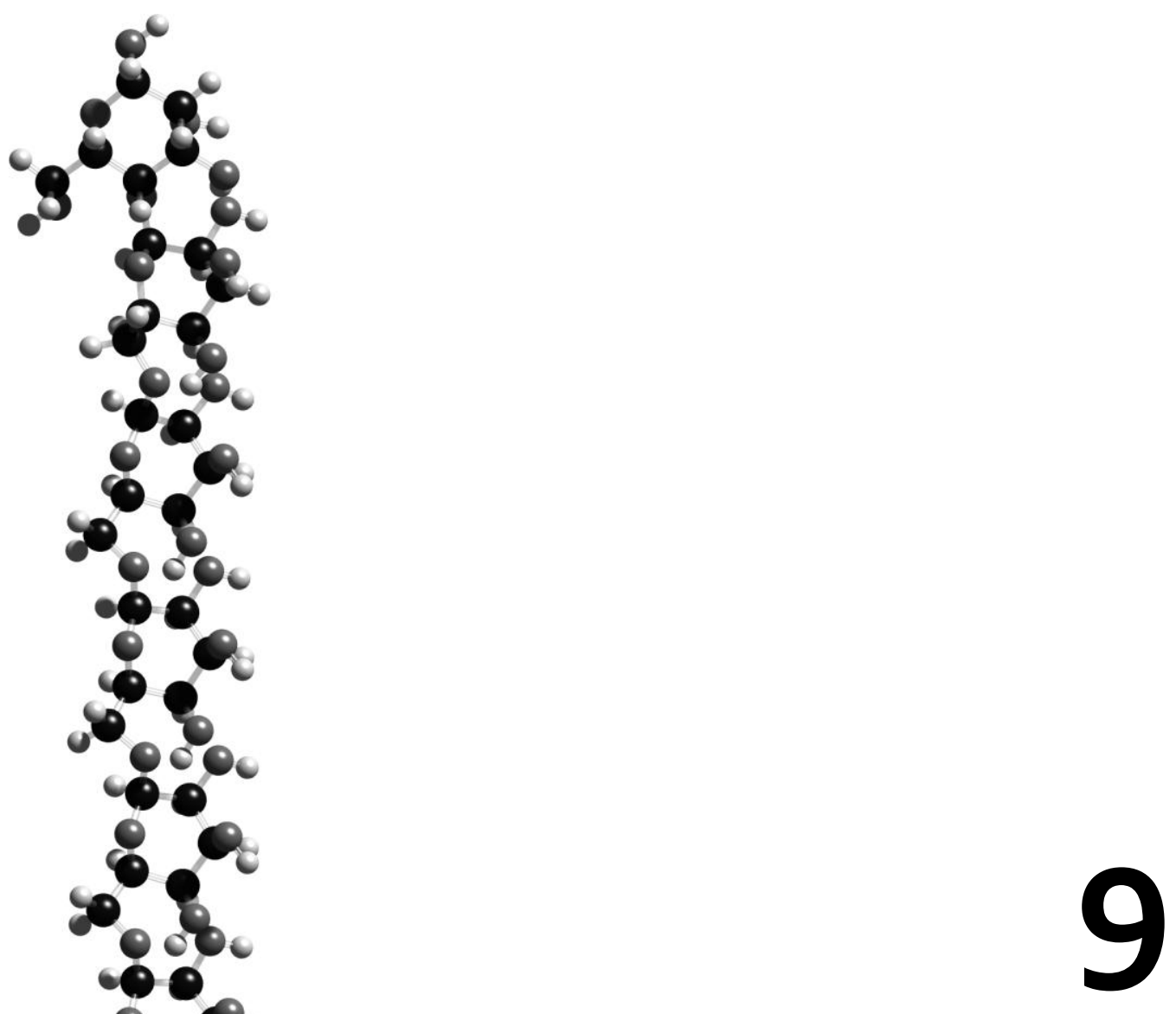

Specific oligosaccharides, low amounts of polyunsaturated fatty acids and a total concept of infant formula ingredients stimulate murine delayedtype hypersensitivity responses

\author{
A.P. Vos \\ K. Knipping \\ M. Balvers \\ B. Stahl \\ G. Boehm \\ J. Garssen \\ L. M'Rabet
}




\section{Abstract}

Specific mixtures of oligosaccharides have been shown previously to induce beneficial immune modulatory effects. In practical applications, such as in infant formula, other immune modulatory ingredients may also be present, including polyunsaturated fatty acids (PUFA), nucleotides, arginine and zinc. Therefore, the combined effect of these ingredients was tested in a murine vaccination model, by measuring the effects on delayed-type hypersensitivity (DTH) responses. An additional experiment was performed to test the effect of low doses of either $n-3$ or $n-6$ PUFAs.

The combination of immune modulating ingredients, including low doses of $n-3$ and $n$ 6 PUFAs, stimulated DTH responses significantly (166\% relative to controls). The PUFAs alone increased the DTH responses as well (140\% relative to controls). In the additional experiment, low-dose supplementation with n-3 PUFAs, but not with n-6 PUFAs, increased the DTH responses mildly but significantly (123\% relative to controls). The serum fatty acid profiles showed that all $n-3$ containing diets resulted in increases in docosahexaenoic acid and other n-3 PUFAs, in parallel to the increases in DTH responses. No consistent effects were observed on arachidonic acid or other $n-6$ PUFAs that correlated with the immunological results.

In conclusion, low-dose n-3 PUFAs, alone or in combination with other immune modulatory ingredients, stimulated Th1 related DTH responses in a murine vaccination model. These results hold promise for the application of these ingredients in infant formula, with the goal to enhance resistance to infections and reduce the incidence of allergies.

\section{Introduction}

Breast-feeding has been reported to result in reduced rates of infections and of occurrence of atopy in infants, compared to bottle-feeding with standard infant formulas without prebiotics (1-5). A prebiotic mixture of short-chain galactooligosaccharides (scGOS) and long-chain fructooligosaccharides (lcFOS) in a 9:1 ratio (Immunofortis) has been described to induce immune modulatory effects that appear to resemble immunological aspects of breast milk on the immune system. In animal experiments, a reduction in allergy-related parameters was observed in animals receiving the scGOS/lcFOS mixture (chapter 8), as well as enhanced T-helper 1 (Th1)- 
related immune responses, an important parameter for resistance to many infectious diseases (chapter 3). Moreover, similar effects were observed in a recent trial with scGOS/lcFOS-fed infants, resulting in significantly reduced parameters of atopy and infection $(6,7)$. Finally, a combination of scGOS/lcFOS and partly unsaturated and partly methylated pectin-derived acidic oligosaccharides (pAOS) showed synergism in stimulating Th1-related immune responses (chapter 4).

When a prebiotic concept is used in an infant formula, it is highly likely to be applied in conjunction with other ingredients that potentially affect the immune function. Polyunsaturated fatty acids (PUFA) of the $n-3$ and $n-6$ type have been described to provide benefits regarding visual functions, cognitive outcomes and cardiovascular parameters in infants (8), providing a rationale for their application. PUFAs have also been described to modulate immune function. A beneficial role has been suggested on allergy development for a long time $(8,9)$. Inverse relationships have been reported between breast milk $n-3$ PUFAs and maternal atopy $(10,11)$, atopy-related parameters in children (12-14) and non-atopic eczema (15). These findings might suggest a role for n-3 PUFAs in the prevention of allergy development. On the other hand, antiinflammatory and even suppressive effects of $n-3$ PUFAs on Th1 related and other immune parameters have been described in literature $(9,16,17)$, making it unclear what the net effect of a combination of PUFAs and prebiotics on the immune system would be.

Other ingredients that might modify immune function in infants include nucleotides, arginine and zinc. Nucleotides might enhance the maturation of the humoral immune system in infants $(18,19)$. Arginine and zinc may positively affect humoral responses and thymus development (20-23); two parameters that have been described to be lowered in formula-fed infants $(24,25)$.

In this study, the combined application of several immune modulatory nutrients was tested on Th1-related delayed-type hypersensitivity (DTH) responsiveness in a murine vaccination model, to test whether the different ingredients interact and their effects possibly cancel each other out. As the literature is ambiguous about the effect of $n-3$ PUFAs on parameters of immune function, an additional experiment was performed to investigate the effects of separate $n-3$ and $n-6$ containing fat blends on DTH responses. The effects of PUFA supplementation on serum phospholipids was analyzed in both experiments. 


\section{Materials and methods}

\section{Mice}

Female, 6 week old C57Bl/6JOlaHsd mice were obtained from Harlan (Horst, The Netherlands) and kept under normal conditions at the animal facility of the Wageningen University, with a $12 \mathrm{~h}$ dark and light cycle and access to food and water ad libitum. The experiments were approved by an independent animal experiments committee (DEC Consult, Bilthoven, The Netherlands).

\section{Diets}

All animals received diets that were based on the semi-purified AIN-93G diet (Research Diet Services, Wijk bij Duurstede, The Netherlands), containing 7\% (w/w) fat (26). The control diet in both experiments consisted of AIN-93G diet, containing a fat blend of $80 \%$ palm oil and $20 \%$ sunflower oil. All supplemented oligosaccharides were exchanged for the same amount of total carbohydrates. All dietary ingredients were mixed into the diet and pressed into pellets. Pellets were stored at $-20^{\circ} \mathrm{C}$ and refreshed weekly in the cages.

In the first experiment, the dosages of test substances in the experimental diets were based on amounts that are suitable for application in infant formula. The active ingredients in the oligosaccharide supplemented group consisted $6 \mathrm{~g} / \mathrm{kg}$ of a mixture of scGOS (Vivinal GOS, Borculo Domo, Zwolle, The Netherlands;) and lcFOS (Raftiline $\mathrm{HP}$, Orafti, Wijchen, The Netherlands;) in a 9:1 ratio (Immunofortis), and $1.5 \mathrm{~g} / \mathrm{kg}$ pectin-derived pAOS were used (kindly provided by Südzucker AG, Mannheim, Germany). A total of $6.5 \mathrm{~g} / \mathrm{kg}$ of carrier carbohydrates was also added and exchanged for total carbohydrates in the diet, which were previously shown not to affect the immune response (27). The nucleotide supplementation group received the control diet, supplemented with $8.9 \mathrm{mg} / \mathrm{kg}$ cytidine monophosphate, $5.5 \mathrm{mg} / \mathrm{kg}$ uridine monophosphate, $8.2 \mathrm{mg} / \mathrm{kg}$ adenosine monophosphate, $2.0 \mathrm{mg} / \mathrm{kg}$ guanosine monophosphate and $3.4 \mathrm{mg} / \mathrm{kg}$ inositol monophosphate. The PUFA supplementation group received the control diet with an alternative fat composition, which contained $1.5 \%$ evening primrose oil, $0.7 \%$ tuna fish oil, $0.8 \%$ arachidonic acid oil, $0.2 \%$ echium oil and $96.8 \%$ of a blend containing palm oil, sunflower oil, coconut oil and rapeseed oil. The fatty acid composition of this diet and the control diet is shown in table 1 . The last group in the first experiment received a diet that combined the described supplementation of oligosaccharides, nucleotides and PUFAs. In addition, $250 \mathrm{mg} / \mathrm{kg}$ arginine and $20 \mathrm{mg} / \mathrm{kg}$ zinc-sulphate was added to this diet. 
In the second experiment, two diets with alternative $n-3$ and $n-6$ containing fat blends were compared to the control diet, containing approximately $6 \%(\mathrm{w} / \mathrm{w})$ eicosapentaenoic acid (EPA) or $\gamma$-linolenic acid (GLA) of total fat. The fat blend in the EPA-supplemented group consisted of $71 \%$ palm oil, $18 \%$ sunflower oil and $11 \%$ Incromega fish oil. The fat blend enriched with GLA contained 59\% palm oil, 15\% sunflower oil and $26 \%$ borage oil. The detailed fatty acid content of these diets is shown in table 1 .

\section{Vaccination protocol and DTH response}

The vaccination protocol has been described in detail in chapter 3. In short, the mice received primary and booster vaccinations, consisting of a subcutaneous (sc) injection of a 1:1 mix of diluted antigen solution (Influvac, Solvay Pharmaceuticals, Weesp, The Netherlands) and adjuvant (Stimune, Cedi-diagnostics, Lelystad, The Netherlands). The booster vaccination was given 21 days after the primary vaccination. The experiments ended 10 days after booster vaccination. Sham vaccinated groups received only adjuvant. Dietary interventions were started 14 days before the primary vaccination and lasted until the end of the experiments. Experimental groups (including the control groups) consisted of 10 animals $(n=10)$; sham groups consisted of

Table 1. The PUFA content of the diets. The control diets were the same in both experiments. In the first experiment, the PUFA supplemented group and the combination group had the same PUFA content (PUFA exp1); the other diets in experiment 1 had the same PUFA content as the control diet.

\begin{tabular}{llrrrr} 
Fatty acid (w/w\% of total FA) & Control & PUFA exp1 & EPA & GLA \\
\hline$C_{1} 8: 2 n 6$ & LA & 19.96 & 12.41 & 17.92 & 24.44 \\
$C_{18}: 3 n 6$ & GLA & 0.00 & 0.52 & 0.00 & 6.01 \\
$C_{20}: 2 n 6$ & EDA & 0.00 & 0.00 & 0.00 & 0.05 \\
$C_{20}: 3 n 6$ & DGLA & 0.00 & 0.01 & 0.00 & 0.00 \\
$C_{20}: 4 n 6$ & AA & 0.00 & 0.18 & 0.18 & 0.00 \\
$C_{22}: 4 n 6$ & DTA & 0.00 & 0.00 & 0.07 & 0.00 \\
$\sum n 6$ & & 19.96 & 13.11 & 18.17 & 30.50 \\
$C_{18}: 3 n 3$ & ALA & & & & \\
$C_{18}: 4 n 3$ & SA & 0.10 & 2.48 & 0.11 & 0.14 \\
$C_{20}: 4 n 3$ & ETA & 0.00 & 0.15 & 0.02 & 0.00 \\
$C_{20}: 5 n 3$ & EPA & 0.00 & 0.00 & 0.17 & 0.00 \\
$C_{22}: 5 n 3$ & $n-3 D P A$ & 0.00 & 0.36 & 6.11 & 0.00 \\
$C_{22}: 6 n 3$ & DHA & 0.00 & 0.12 & 0.94 & 0.00 \\
$\sum n 3$ & & 0.00 & 1.39 & 2.35 & 0.00 \\
\hline
\end{tabular}


3 animals $(n=3)$. Sham groups were not used for statistical analyses, but included to demonstrate the specificity of the DTH response to the vaccine.

DTH reactions were induced 9 days after booster vaccination, by sc injection of $25 \mu \mathrm{L}$ Influvac into the ear pinnae of both ears. Ear thickness was measured in duplicate before antigen challenge, as well as 24 hours afterwards, with a digital micrometer (Mitutoyo Digimatic 293561, Veenendaal, The Netherlands). The DTH response was calculated by subtraction of the basal ear thickness from the value at 24 hours after challenge. At the end of the experiment, blood samples were drawn. After centrifugation, serum samples were collected and stored at $-80{ }^{\circ} \mathrm{C}$ until further analysis.

\section{Fatty acid analysis of phospholipids}

Fatty acid extraction from serum samples was performed according to Bligh and Dyer (28). Phospholipids were separated by using Bond-Elut $\AA$ solid phase extraction columns and the Vac-Elut SPS $24^{\mathrm{TM}}$ system. Phospholipid extracts were converted to their methyl esters by using $14 \% \mathrm{BF}_{3}$ in methanol at $100^{\circ} \mathrm{C}$ for $60 \mathrm{~min}$. After hexane extraction derivatized phospholipids were dissolved in iso-octane and analyzed by gas chromatography (GC) with a capillary column (50m x 0.25mm, CP-SIL88-fame). Peaks were identified by commercial reference standards. Fatty acid analysis was performed on individual serum samples; statistics were calculated per experimental group.

\section{Statistical analysis}

Statistical calculations were performed in SPSS version 15.0.0 and Graphpad software. Statistical differences between DTH responses of test groups versus a single control group were analyzed by ANOVA and post-hoc Dunnett's test. For analysis of serum phospholipid PUFA fractions, post-hoc Bonferroni analysis was performed per group of analytes. Three analyte groups were analyzed: total n-3 and n-6 PUFAs, all measurable separate $n$ - 3 PUFAs and all measurable separate $n-6$ PUFAs. P-values $<0.05$ were considered significant. Data are represented as means \pm standard error of the mean (SEM). 


\section{Results}

Dietary intervention with the specific mixture of oligosaccharides $(136.4 \pm 6.9 \mu \mathrm{m})$ and with PUFAs $(114.7 \pm 6.2 \mu \mathrm{m})$ significantly stimulated DTH responses after vaccination compared to control animals $(81.5 \pm 5.0 \mu \mathrm{m}$; see Fig. 1$)$. In addition, the combined diet containing all test substances also significantly enhanced the DTH response $(135.5 \pm 6.5$ $\mu \mathrm{m})$ compared to the animals receiving control diet. No differences between diets were observed with regard to animal weight or feed intake (see table 2). The phospholipid fatty acid composition of the sera was analyzed from mice that were fed with the control diet, the PUFA-enriched diet and the combination diet. As the results show in table 3, the PUFA-enriched diet significantly increased total $n-6$ PUFAs as well as arachidonic acid (AA) in serum; the same trends were observed in the combination diet. A downstream fatty acid in the $n-6$ elongation pathway that was not present in any of the diets, $n-6$ docosapentaenoic acid (n-6 DPA), was significantly reduced in both PUFA supplemented diets. The total fraction of $n-3$ PUFAs in serum phospholipids was significantly increased in the PUFA supplemented diets compared to the control diet. This was caused mainly by an increase in docosahexaenoic acid

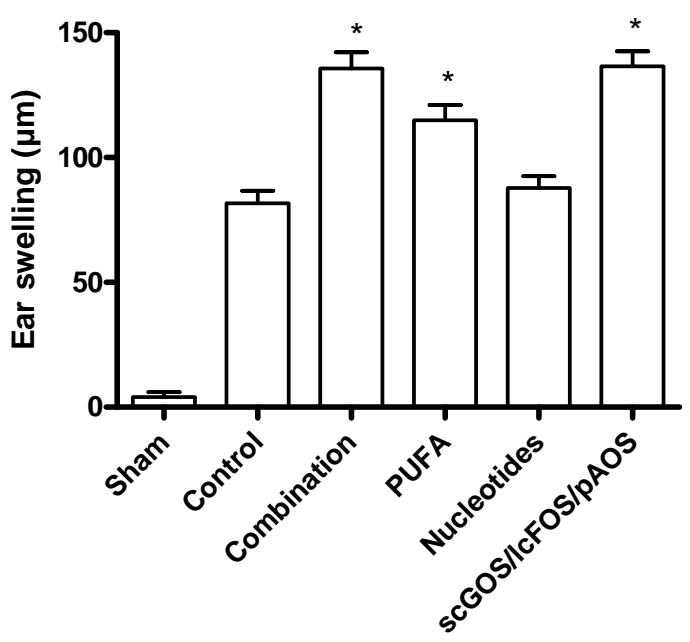

Figure 1. The effects of PUFAs, oligosaccharides and other immune modulatory ingredients on delayedtype hypersensitivity responses. Dietary supplementation with low-dose PUFAs, nucleotides, scGOS/lcFOS/pAOS or a combination of these ingredients together with low levels of arginine and zinc was tested in a murine vaccination model. DTH responses were measured by an increase in ear thickness. *Indicates $p<0.05$ compared with the control group. Data in all graphs represent group means and error bars indicate SEM. 
Table 2. Animals weights and weekly feed intake. Animals and feed intake were weighed weekly. Animal weights are shown from the end of the experiment. Data are represented as mean \pm SEM. No significant differences were observed between groups within an experiment.

\begin{tabular}{|c|c|c|c|c|c|}
\hline \multirow[t]{2}{*}{ Experiment } & \multirow{2}{*}{$\begin{array}{l}\text { Group } \\
\text { Control }\end{array}$} & \multicolumn{2}{|c|}{$\begin{array}{c}\text { Animal weight }(\mathrm{g}) \\
\text { at end of } \\
\text { experiment }\end{array}$} & \multicolumn{2}{|c|}{$\begin{array}{l}\text { Weekly feed intake } \\
\text { (g) per animal }\end{array}$} \\
\hline & & $20.59 \pm$ & 0.39 & $16.83 \pm$ & 0.49 \\
\hline \multirow[t]{5}{*}{ Experiment 1} & Combination & $20.29 \pm$ & 0.47 & $16.36 \pm$ & 0.26 \\
\hline & PUFA & $20.63 \pm$ & 0.38 & $16.34 \pm$ & 0.39 \\
\hline & Nucleotides & $20.01 \pm$ & 0.13 & $16.77 \pm$ & 0.36 \\
\hline & scGOS/lcFOS/pAOS & $20.78 \pm$ & 0.57 & $17.62 \pm$ & 0.41 \\
\hline & Control & $19,72 \pm$ & 0,32 & $17,86 \pm$ & 0,42 \\
\hline \multirow[t]{2}{*}{ Experiment 2} & EPA & $20,32 \pm$ & 0,38 & $18,65 \pm$ & 0,29 \\
\hline & GLA & $19,96 \pm$ & 0,27 & $18,31 \pm$ & 0,40 \\
\hline
\end{tabular}

(DHA). No significant differences were observed between the combination and the PUFA groups for any of the fatty acids.

To further investigate effects of the enrichment of different PUFAs on the DTH response, a second experiment was performed in which n-3 (EPA) or n-6 (GLA) enriched diets were tested in comparison to the control diet. In this experiment,

Table 3. PUFA composition of serum phospholipids in mice from the first experiment. Data are expressed as mean \pm SEM; * indicates $p<0.05$ compared control diet. No significant differences were found between the combination and PUFA diets.

\begin{tabular}{|c|c|c|c|c|c|c|c|c|c|c|c|}
\hline \multicolumn{2}{|c|}{ Fatty acid ( $\%$ of total FA) } & \multicolumn{2}{|c|}{ Control } & \multicolumn{4}{|c|}{ Combination } & \multicolumn{4}{|c|}{ PUFA } \\
\hline$C_{18: 2 n 6}$ & LA & $13.83 \pm$ & 0.52 & $15 \cdot 55$ & \pm & 0.31 & & 16.39 & \pm & 1.13 & \\
\hline$C_{18: 3 n 6}$ & GLA & $0.00 \pm$ & 0.00 & 0.07 & \pm & 0.02 & * & 0.05 & \pm & 0.02 & \\
\hline C20:2n6 & EDA & $0.04 \pm$ & 0.02 & 0.10 & \pm & 0.03 & & 0.15 & \pm & 0.03 & \\
\hline C20:3n6 & DGLA & $2.44 \pm$ & 0.08 & 2.11 & \pm & 0.06 & & 2.23 & \pm & 0.09 & \\
\hline C20:4n6 & AA & $7.24 \pm$ & 0.25 & 8.56 & \pm & 0.55 & & 9.09 & \pm & 0.34 & * \\
\hline$C_{22: 5 n 6}$ & $n-6$ DPA & $1.38 \pm$ & 0.02 & 0.31 & \pm & 0.03 & * & 0.34 & \pm & 0.04 & * \\
\hline$\sum n 6$ & & $25.01 \pm$ & 0.45 & 26.78 & \pm & 0.52 & & 28.40 & \pm & 1.18 & * \\
\hline C20:5n3 & EPA & $0.00 \pm$ & 0.00 & 0.08 & \pm & 0.03 & & 0.21 & \pm & 0.06 & * \\
\hline C22:5n3 & $n-3$ DPA & $0.00 \pm$ & 0.00 & 0.12 & \pm & 0.02 & * & 0.26 & \pm & 0.08 & * \\
\hline C22:6n3 & DHA & 2.77 & 0.09 & 7.01 & \pm & 0.22 & * & 6.62 & \pm & 0.68 & * \\
\hline$\sum \mathrm{n}_{3}$ & & $2.77 \pm$ & 0.09 & 7.21 & \pm & 0.23 & * & 7.16 & \pm & 0.63 & * \\
\hline
\end{tabular}


significantly higher DTH responses were measured in mice that received the EPAenriched diet $(152.7 \mu \mathrm{m} \pm 7.8)$ than in mice that received the control diet $(124.1 \mu \mathrm{m} \pm$ 8.6). In contrast, the GLA-enriched diet had no effect on the DTH response $(119.5 \mu \mathrm{m} \pm$ 7.7; Fig. 2). The proportions of fatty acids in serum phospholipids were differentially affected by dietary intervention with EPA- and GLA-enriched diets, as shown in table 4. The EPA diet resulted in significantly lower proportions of all $n-6$ PUFAs. The largest reductions were measured on the levels of AA and n- 6 DPA. The EPA enriched diet increased the total fraction of $n-3$ PUFAs. On the single fatty acid level, the proportions of EPA and its metabolites were significantly increased; the change in DHA levels was the largest. The GLA-enriched diet resulted in elevated proportions of most $n-6$ PUFAs, most notably affecting GLA and AA, resulting in a significant increase of total serum n6 PUFAs. No statistical differences were found in $n$-3 PUFAs between the GLA enriched diet and the control diet. The proportions of $\alpha$-linolenic acid and stearidonic acid were undetectible in the majority of the samples in both experiments.

\section{Discussion}

In accordance with the results in chapters 3 and 4, a combination of scGOS/lcFOS and pAOS significantly enhanced vaccine-specific DTH responses. Surprisingly, diets that were enriched with low levels of $n-3$ plus n-6 PUFAs, or n-3 PUFAs alone, also stimulated Th1-related DTH responses mildly compared to the control diet, which

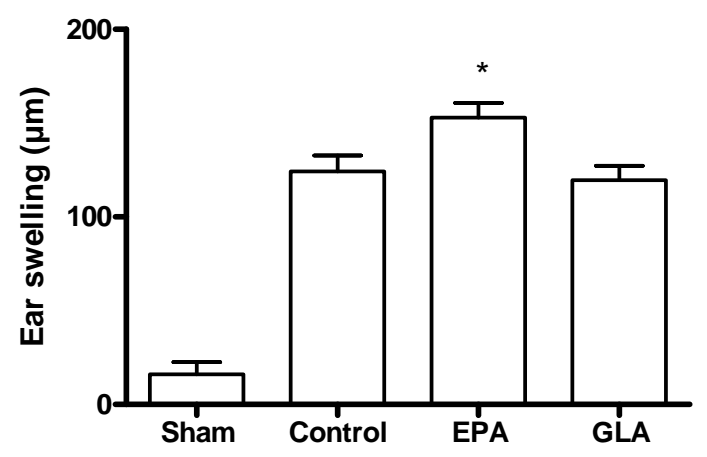

Figure 2. The effects of low dose $n-3$ or n-6 PUFAs on delayed-type hypersensitivity responses. Dietary supplementation with low-dose PUFAs, nucleotides and scGOS/lcFOS/pAOS were tested alone or in combination, together with low levels of arginine and zinc. DTH responses were measured by an increase in ear thickness. *Indicates $p<0.05$ compared with the control group. Data in all graphs represent group means and error bars indicate SEM. 
Table 4. PUFA composition of serum phospholipids in mice from the second experiment. Data are expressed as mean \pm SEM; * indicates $\mathrm{p}<0.05$ compared control diet. All fatty acids differed significantly between the EPA and GLA groups except for LA and EDA.

\begin{tabular}{|c|c|c|c|c|c|c|c|c|c|c|c|c|}
\hline \multicolumn{2}{|c|}{ Fatty acid ( $\%$ of total FA) } & \multicolumn{3}{|c|}{ Control } & \multicolumn{4}{|c|}{ EPA } & \multicolumn{4}{|c|}{ GLA } \\
\hline$C_{18: 2 n 6}$ & LA & 17.75 & \pm & 0.80 & $15 \cdot 30$ & \pm & 0.33 & & 14.22 & \pm & 0.39 & * \\
\hline$C_{18}: 3 n 6$ & GLA & 0.09 & \pm & 0.01 & 0.00 & \pm & 0.00 & * & 0.36 & \pm & 0.02 & * \\
\hline C20:2n6 & EDA & 0.24 & \pm & 0.02 & 0.04 & \pm & 0.02 & * & 0.12 & \pm & 0.01 & * \\
\hline C20:3n6 & DGLA & 2.82 & \pm & 0.15 & 1.24 & \pm & 0.05 & * & 2.98 & \pm & 0.14 & \\
\hline$C 20: 4 n 6$ & $\mathrm{AA}$ & 9.20 & \pm & 0.57 & 2.55 & \pm & 0.09 & * & 14.06 & \pm & 0.46 & * \\
\hline C22:5n6 & n-6 DPA & 2.43 & \pm & 0.26 & 0.01 & \pm & 0.01 & * & 3.02 & \pm & 0.14 & \\
\hline$\sum n 6$ & & 32.90 & \pm & 0.69 & 19.15 & \pm & 0.35 & * & 35.28 & \pm & 0.44 & * \\
\hline C20:5n3 & EPA & 0.08 & \pm & 0.09 & 3.70 & \pm & 0.09 & * & 0.00 & \pm & 0.00 & \\
\hline C22:5n3 & $n-3$ DPA & 0.15 & \pm & 0.08 & 1.41 & \pm & 0.06 & * & 0.12 & \pm & 0.00 & \\
\hline C22:6n3 & $\mathrm{DHA}$ & 2.88 & \pm & 0.08 & 12.91 & \pm & 0.21 & * & 3.33 & \pm & 0.13 & \\
\hline$\sum \mathrm{n}_{3}$ & & 3.18 & \pm & 0.20 & 18.02 & \pm & 0.24 & * & 3.46 & \pm & 0.13 & \\
\hline
\end{tabular}

contained no PUFAs except for the essential PUFAs linoleic acid and $\alpha$-linolenic acid. The currently used dosages of oligosaccharides, PUFAs and a combination of immune modulating ingredients did not result in opposing effects on the DTH responses. The fact that no additive effect was observed in the combined group might have been caused by shared parts of the underlying immune-modulatory pathways of PUFAs and oligosaccharides. However, given the clear differences between these ingredients with regard to intestinal and metabolic effects, it is difficult to hypothesize what this common factor in the immune-modulatory mechanism could be. No effects of nucleotide supplementation were observed on the DTH response. A stimulation of circulating IgM and IgA antibodies was reported in nucleotide-supplemented preterm infants (18). Together, this suggests that nucleotide supplementation affects humoral rather than cell-mediated immune responses.

It is interesting to compare the serum fatty acid profiles from both experiments. It has been shown that these are highly correlated with the profiles of leukocyte membrane phospholids (29). The diets that contained $n$-3 PUFAs induced significant alterations in the proportions of many serum phospholipid PUFAs in both experiments, as well as significantly increased DTH responses. The distribution of the different $n-3$ PUFAs in serum phospholipids did not correspond directly with the distributions of $n$-3 PUFAs in the diets: most dietary EPA was metabolized in the animals, shown by the 
accumulation of DHA in serum. In contrast, no consistent effects were observed on AA in both experiments that could explain the increased DTH responses. Therefore, it is tempting to speculate that the increases in DHA caused the increase in DTH response. Although DHA, in contrast to EPA, is not a substrate for eicosanoid production, it decreases the production of AA-derived eicosanoids (30). DHA itself can be metabolized to a number of other immunologically active mediators such as D-series resolvins and protectins (30). In addition, DHA and EPA have been described to remodel the phospholipid composition of $\mathrm{T}$ cell lipid rafts, which are membrane structures that play an important role in T cell signaling and activation (31). Although it is not yet clear how a low dose of $n-3$ PUFAs can lead to increased DTH responses, it is possible that these mechanisms played a role in the observed effects.

The increase in DTH responses that was induced in $n-3$ supplemented groups suggests a stimulation of Th1-related immune responses. Inhibitory effects of $n$-3 PUFAs on Th2related responses have been described previously. Aerosolized DHA reduced lung eosinophil infiltration in a murine allergy model (32). In addition, dietary $n-3$ PUFAs inhibited allergic responses in mice (33) and enhanced oral tolerance induction in rats (34). As Th1 and Th2-related responses are thought to be reciprocally regulated, these findings are in keeping with an increase in Th1 responses.

In contrast, many reports in literature have shown suppressive effects of PUFAs on Tcell responses, most notably from $n-3$ PUFAs on Th1-related responses (35-37). In addition, a high dose of $n-3$ plus $n-6$ PUFAs was shown to reduce vaccine-specific DTH responses in healthy mice (38). The dosages of the applied n-3 PUFAs might play a key role in the observed differences. Dosages of fish oil in the diets range mostly between 4$20 \%(\mathrm{w} / \mathrm{w})$ in literature. In this experiment, the EPA group received a net amount of only $0.8 \%(\mathrm{w} / \mathrm{w})$ of fish oil in the total diet. Yet, significant changes of fatty acid profiles were observed, as well as functional changes. We argue that these low doses are relevant, as the test diets featured proportions of $n-3$ and $n-6$ PUFAs, relative to total dietary fat, that are relevant for application in infant formula and are within the ranges observed in breast milk (39).

Apparently contradictory effects of pre- and probiotic agents have been reported in literature, including stimulatory effects on Th1-related immune parameters and ameliorative effects on inflammatory or autoimmune conditions. These diverse results have been hypothesized to be due to beneficial effects of pre- and probiotics on immune regulation, caused by the modulation of the intestinal microbiota $(40,41)$. The current results show a similar paradox in the immunological effects of $n$ - 3 PUFAs, as the stimulatory effect on the DTH response is in apparent contradiction with the anti- 
inflammatory effects of $n$-3 PUFAs that have been widely described in literature. On a functional level, it could therefore be hypothesized that n-3 PUFAs may modulate immune-regulatory processes as well. However, as mentioned earlier, the differences between PUFAs and pre- and probiotics make it difficult to hypothesize a common biochemical pathway or mechanism.

In conclusion, the stimulatory effect of a specific mixture of oligosaccharides on the cellular immune response after vaccination in a murine model was not negatively affected by simultaneous supplementation of PUFAs and other immune modulatory ingredients. Moreover, low-dose n-3 PUFA supplementation alone enhanced Th1related immune responses as well. For the potential application of these ingredients in infant formula, the results suggest that scGOS/lcFOS/pAOS, low-dose PUFAs and other immune-modulatory nutrients in infant formulas may help to increase resistance to infections and reduce the development of allergies in infants. Well-designed clinical trials need to be conducted to prove this hypothesis.

\section{References}

1. Friedman, N.J. and R.S. Zeiger, The role of breast-feeding in the development of allergies and asthma. J Allergy Clin Immunol, 2005. 115(6): p. 1238-48.

2. Gdalevich, M., D. Mimouni, and M. Mimouni, Breast-feeding and the risk of bronchial asthma in childhood: a systematic review with meta-analysis of prospective studies. J Pediatr, 2001. 139(2): p. 261-6.

3. van Odijk, J., I. Kull, M.P. Borres, P. Brandtzaeg, U. Edberg, L.A. Hanson, A. Host, M. Kuitunen, S.F. Olsen, S. Skerfving, J. Sundell, and S. Wille, Breastfeeding and allergic disease: a multidisciplinary review of the literature (1966-2001) on the mode of early feeding in infancy and its impact on later atopic manifestations. Allergy, 2003. 58(9): p. 833-43.

4. Kramer, M.S. and R. Kakuma, Optimal duration of exclusive breastfeeding. Cochrane Database Syst Rev, 2002(1): p. CDoo3517.

5. Rossum van, C.T.M., F.L. Buchner, and J. Hoekstra, Quantification of health effects of breast feeding, review of the literature and model simulation, 350040001, Editor. 2005, RIVM: Bilthoven. p. 108.

6. Arslanoglu, S., G. Moro, J. Schmitt, and G. Boehm, Early dietary intervention with a mixture of prebiotic oligosaccharides reduces the incidence of allergy associated symptoms and infections during the first 2 years of life. J Pediatr Gastroenterol Nutr, 2007. 44(S1): p. e217.

7. Moro, G., S. Arslanoglu, B. Stahl, J. Jelinek, U. Wahn, and G. Boehm, A mixture of prebiotic oligosaccharides reduces the incidence of atopic dermatitis during the first six months of age. Arch Dis Child, 2006. 91(10): p. 814-9.

8. Fleith, M. and M.T. Clandinin, Dietary PUFA for preterm and term infants: review of clinical studies. Crit Rev Food Sci Nutr, 2005. 45(3): p. 205-29.

9. Prescott, S.L. and P.C. Calder, N-3 polyunsaturated fatty acids and allergic disease. Curr Opin Clin Nutr Metab Care, 2004. 7(2): p. 123-9. 
10. $Y u, G ., K$. Duchen, and B. Bjorksten, Fatty acid composition in colostrum and mature milk from nonatopic and atopic mothers during the first 6 months of lactation. Acta Paediatr, 1998. 87(7): p. 729-36.

11. Wright, S. and C. Bolton, Breast milk fatty acids in mothers of children with atopic eczema. Br J Nutr, 1989. 62(3): p. 693-7.

12. Reichardt, P., D. Muller, U. Posselt, B. Vorberg, U. Diez, U. Schlink, W. Reuter, and M. Borte, Fatty acids in colostrum from mothers of children at high risk of atopy in relation to clinical and laboratory signs of allergy in the first year of life. Allergy, 2004. 59(4): p. 394-400.

13. Duchen, K., R. Casas, M. Fageras-Bottcher, G. Yu, and B. Bjorksten, Human milk polyunsaturated long-chain fatty acids and secretory immunoglobulin A antibodies and early childhood allergy. Pediatr Allergy Immunol, 2000. 11(1): p. 29-39.

14. Duchen, K., G. Yu, and B. Bjorksten, Atopic sensitization during the first year of life in relation to long chain polyunsaturated fatty acid levels in human milk. Pediatr Res, 1998. 44(4): p. 478-84.

15. Oddy, W.H., S. Pal, M.M. Kusel, D. Vine, N.H. de Klerk, P. Hartmann, P.G. Holt, P.D. Sly, P.R. Burton, F.J. Stanley, and L.I. Landau, Atopy, eczema and breast milk fatty acids in a high-risk cohort of children followed from birth to 5 yr. Pediatr Allergy Immunol, 2006. 17(1): p. 4-10.

16. Calder, P.C., Polyunsaturated fatty acids, inflammation, and immunity. Lipids, 2001. 36(9): p. 100724 .

17. Calder, P.C., Dietary modification of inflammation with lipids. Proc Nutr Soc, 2002. 61(3): p. 345-58.

18. Navarro, J., J. Maldonado, E. Narbona, A. Ruiz-Bravo, J.L. Garcia Salmeron, J.A. Molina, and A. Gil, Influence of dietary nucleotides on plasma immunoglobulin levels and lymphocyte subsets of preterm infants. Biofactors, 1999. 10(1): p. 67-76.

19. Martinez-Augustin, O., J.J. Boza, J.I. Del Pino, J. Lucena, A. Martinez-Valverde, and A. Gil, Dietary nucleotides might influence the humoral immune response against cow's milk proteins in preterm neonates. Biol Neonate, 1997. 71(4): p. 215-23.

20. Roy, S.K., Effect of zinc supplementation in patients with acute and persistent diarrhoea. Glimpse, 1991. 13(3): p. 2.

21. Raqib, R., S.K. Roy, M.J. Rahman, T. Azim, S.S. Ameer, J. Chisti, and J. Andersson, Effect of zinc supplementation on immune and inflammatory responses in pediatric patients with shigellosis. Am J Clin Nutr, 2004. 79(3): p. 444-50.

22. Fabris, N. and E. Mocchegiani, Arginine-containing compounds and thymic endocrine activity. Thymus, 1992. 19 Suppl 1: p. S21-30.

23. Bansal, V. and J.B. Ochoa, Arginine availability, arginase, and the immune response. Curr Opin Clin Nutr Metab Care, 2003. 6(2): p. 223-8.

24. Hasselbalch, H., D.L. Jeppesen, M.D. Engelmann, K.F. Michaelsen, and M.B. Nielsen, Decreased thymus size in formula-fed infants compared with breastfed infants. Acta Paediatr, 1996. 85(9): p. 1029-32.

25. Hasselbalch, H., M.D. Engelmann, A.K. Ersboll, D.L. Jeppesen, and K. Fleischer-Michaelsen, Breastfeeding influences thymic size in late infancy. Eur J Pediatr, 1999. 158(12): p. 964-7.

26. Reeves, P.G., F.H. Nielsen, and G.C. Fahey, Jr., AIN-93 purified diets for laboratory rodents: final report of the American Institute of Nutrition ad hoc writing committee on the reformulation of the AIN-76A rodent diet. J Nutr, 1993. 123(11): p. 1939-51.

27. Vos, A.P., M. Haarman, A. Buco, M. Govers, J. Knol, J. Garssen, B. Stahl, G. Boehm, and L. M'Rabet, A specific prebiotic oligosaccharide mixture stimulates delayed-type hypersensitivity in a murine influenza vaccination model. Int Immunopharmacol, 2006. 6(8): p. 1277-86.

28. Bligh, E.D. and W.J. Dyer, A rapid method for total lipid extraction and purification. Can. Journal Biochem Physiol, 1959. 37: p. 911-917. 
29. Dooper, M.M.B.W., A.P. Vos, J.d. Groot, A.J. Masterson, R.J. Scheper, and L. M'Rabet, The effects of dietary gamma-linolenic, dihomo-gamma-linolenic, arachidonic and eicosapentaenoic acids on B16melanoma growth and metastasis in mice, in Immune-modulating effects of polyunsaturated fatty acids: focus on dihomo-gamma-linolenic acid. Thesis. 2004: Nijmegen, the Netherlands. p. 65-76.

30. Calder, P.C., n-3 polyunsaturated fatty acids, inflammation, and inflammatory diseases. Am J Clin Nutr, 2006. 83(6 Suppl): p. 1505S-1519S.

31. Fan, Y.Y., D.N. McMurray, L.H. Ly, and R.S. Chapkin, Dietary (n-3) polyunsaturated fatty acids remodel mouse T-cell lipid rafts. J Nutr, 2003. 133(6): p. 1913-20.

32. Yokoyama, A., T. Hamazaki, A. Ohshita, N. Kohno, K. Sakai, G.D. Zhao, H. Katayama, and K. Hiwada, Effect of aerosolized docosahexaenoic acid in a mouse model of atopic asthma. Int Arch Allergy Immunol, 2000. 123(4): p. 327-32.

33. Watanabe, S., N. Sakai, Y. Yasui, Y. Kimura, T. Kobayashi, T. Mizutani, and H. Okuyama, A high alpha-linolenate diet suppresses antigen-induced immunoglobulin $E$ response and anaphylactic shock in mice. J Nutr, 1994. 124(9): p. 1566-73.

34. Korotkova, M., E. Telemo, L.A. Hanson, and B. Strandvik, Modulation of neonatal immunological tolerance to ovalbumin by maternal essential fatty acid intake. Pediatr Allergy Immunol, 2004. 15(2): p. 112-22.

35. Zhang, P., W. Kim, L. Zhou, N. Wang, L.H. Ly, D.N. McMurray, and R.S. Chapkin, Dietary fish oil inhibits antigen-specific murine Th1 cell development by suppression of clonal expansion. J Nutr, 2006. 136(9): p. 2391-8.

36. Zhang, P., R. Smith, R.S. Chapkin, and D.N. McMurray, Dietary (n-3) polyunsaturated fatty acids modulate murine Th1/Th2 balance toward the Th2 pole by suppression of Th1 development. J Nutr, 2005. 135(7): p. 1745-51.

37. Switzer, K.C., Y.Y. Fan, N. Wang, D.N. McMurray, and R.S. Chapkin, Dietary n-3 polyunsaturated fatty acids promote activation-induced cell death in Th1-polarized murine CD4+ T-cells. J Lipid Res, 2004. 45(8): p. 1482-92.

38. Crevel, R.W., J.V. Friend, B.F. Goodwin, and W.E. Parish, High-fat diets and the immune response of $C_{57}$ Bl mice. Br J Nutr, 1992.67(1): p. 17-26.

39. Brenna, J.T., B. Varamini, R.G. Jensen, D.A. Diersen-Schade, J.A. Boettcher, and L.M. Arterburn, Docosahexaenoic and arachidonic acid concentrations in human breast milk worldwide. Am J Clin Nutr, 2007. 85(6): p. 1457-64.

40. Matsuzaki, T., Y. Nagata, S. Kado, K. Uchida, I. Kato, S. Hashimoto, and T. Yokokura, Prevention of onset in an insulin-dependent diabetes mellitus model, NOD mice, by oral feeding of Lactobacillus casei. Apmis, 1997. 105(8): p. 643-9.

41. Guarner, F., R. Bourdet-Sicard, P. Brandtzaeg, H.S. Gill, P. McGuirk, W. van Eden, J. Versalovic, J.V. Weinstock, and G.A. Rook, Mechanisms of disease: the hygiene hypothesis revisited. Nat Clin Pract Gastroenterol Hepatol, 2006. 3(5): p. 275-84. 


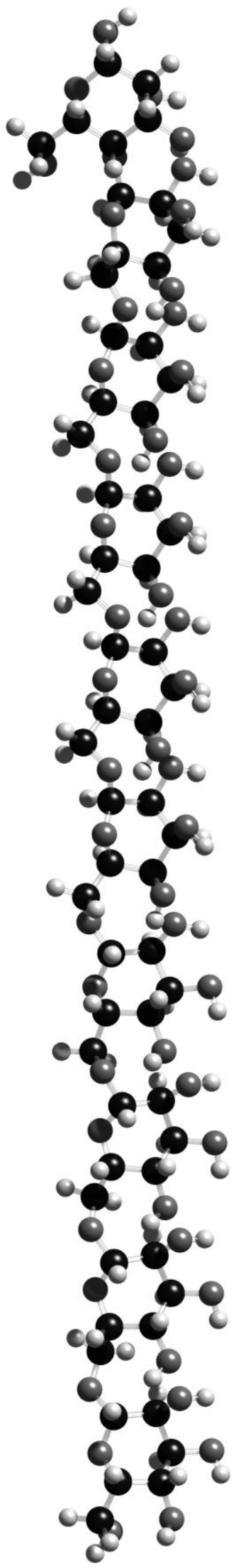

General discussion 


\section{Modulation of systemic immunity by prebiotic oligosaccharides}

The main function of the immune system is the protection of the host organism against infection by pathogens. It is a highly interactive system, thought to be compartmentalized into the mucosal and the systemic parts. Although there is a high degree of crosstalk, many differences have been described between both parts, including differences in cell populations, homing behavior and the classes of predominant antibody production. In addition, there is a degree of separation in mucosal and systemic immune responses (1). In accordance with this concept, modulatory effects of several orally applied probiotic bacteria or prebiotic agents have been reported on mucosal immune parameters (2-4). Previously, a specific mixture of scGOS/lcFOS was shown to induce prebiotic effects and modulate mucosal IgA responses in human infants (5-7). Dietary application of this mixture, alone or in combination with pAOS, was shown in this thesis to modulate systemic immune parameters in various mouse models (chapters 3-5 and 7-8).

Increased DTH responses (chapters 3-5) and suppressed allergic inflammation responses to OVA (chapter 8) indicate changes in immune function that are consistent with a shift in the Th1/Th2 balance, stimulating Th1-related immunity and/or suppressing Th2-related responses. Indeed, a reduction in the ex-vivo production of Th2-related cytokines was observed in pAOS-supplemented animals in parallel to increased DTH responses (chapter 4), although this effect on cytokine production could not be demonstrated in other vaccination experiments. Yet, the concept of a shift in the Th1/Th2 balance alone is not sufficient to describe all observed immune modulatory effects of the oligosaccharide mixtures. The reduction of early MCMV replication, described in chapter 7 , suggests a modulation of innate immune function that may be due to increased NK activity. In accordance, a dose-dependent increase in splenocyte NK activity was observed in senescence-accelerated SAMP8 mice, supplemented with various doses of scGOS/lcFOS/pAOS (unpublished observations). Th1 and NK cells have several things in common, including the fact that the cytokines IL-12, IL-18 and IFN- $\gamma$ play important roles in their polarization and/or activity. Therefore, modulation of these cytokines could play a role in the observed effects, but it is not clear where, when and by what cell type(s) this effect could be actuated.

The oligosaccharide mixtures that were studied in this thesis are primarily aimed at applications in baby food. Therefore, immune-modulatory effects of a novel baby food 
concept were investigated as well. For this purpose, the net effect of all potential immune-modulating components from this concept were tested in the influenza vaccination model, with an additional focus on the effects of $n-3$ and n-6 PUFAs. The combination of these components, including low doses of PUFAs, induced similar stimulatory effects on the DTH response as the oligosaccharides alone (chapter 9). Overall, the immune-modulatory effects of scGOS/lcFOS and pAOS that were observed in several mice models are promising for the development of new infant formulas, aimed at minimizing the development of allergies and maximizing the resistance to infections.

With regard to the underlying mechanism, the observations throughout this thesis raise two questions: what immunological cell types are affected by the modulatory effects of the oligosaccharides, and since the oligosaccharides are applied via a mucosal organ system, how does dietary application lead to modulation of systemic immunity? Obviously, these are big questions that cannot be answered conclusively on the basis of the experimental results in thesis. Still, it is interesting to make an attempt at general 'pattern recognition' by mirroring the results to the literature.

\section{The role of the intestinal microbiota in the observed effects}

As argued in chapter 2, it is possible that dietary oligosaccharides mediate immune modulatory effects by both microbiota-dependent and -independent mechanisms. In chapters 3-5, various associations have been observed between microbiota-related parameters and increases in DTH responses. scGOS/lcFOS, with or without pAOS, repeatedly stimulated the percentages of intestinal bifidobacteria and lactobacilli. However, other oligosaccharides and oligosaccharide mixtures led to a variety of results in chapters 3 and 4; prebiotic effects in fecal or colon samples were observed with and without simultaneous effects on the DTH response. Moreover, a stimulation of DTH responses was observed with increasing doses of pAOS (as a single component), without a measurable effect on colonic bifidobacteria or lactobacilli. However, it was found in chapter 5 that vaccination responses appeared to be modulated shortly after the primary vaccination, and that the proportion of lactobacilli in the cecum correlated best with DTH responses. These results suggest that the analyses in chapters 3 and 4, performed on colon and fecal samples that were isolated at the end of a 4-week vaccination schedule, may not have been relevant for studying the role of the microbiota in the observed effects on the DTH response.

A weak but significant correlation was found between lactobacilli in the cecum and increased DTH responses (chapter 5). The necessity of a prefeeding period for the 
immune modulatory effect to occur indicates a non-instantaneous effect, which is suggestive of a microbiota-dependent effect that takes some time to develop. Additionally, it is known that DTH responses and NK activity are suppressed in germfree mice $(8,9)$, and dietary application of various probiotic bacteria have been reported to enhance NK activity $(10,11)$, increase DTH responses (12) and reduce eosinophilic airway inflammation (13) in murine models. Together, these data indicate that bacterial effects, in a broad sense, are sufficient to induce the observed immunemodulatory effects that are described in this thesis. However, it remains unclear whether the prebiotic effects on the microbiota were also necessary for all observed immune-modulatory effects.

No evidence for oligosaccharide-induced microbiota-independent effects was found in vitro, but only a limited set of assays was performed (chapter 6). A more extensive set of in vitro experiments is necessary to investigate relevant potential effects on DCs, T cells and possibly natural killer T (NKT) cells (discussed below).

In summary, no convincing evidence was found in this thesis for microbiotaindependent immune-modulatory effects, while increases in the percentages of intestinal bifidobacteria and lactobacilli were repeatedly observed in scGOS/lcFOS(/pAOS)-fed animals. However, the strength of the correlation that was found between these bacterial parameters and the DTH response suggests that additional research, aimed at the identification of more specific parameters that show a strong quantitative relationship with immune modulatory effects, may help to unravel details of the causative mechanism. Clarification of this issue might also provide an explanation for the differences that were observed on DTH responses between various types of supplemented oligosaccharides.

\section{The potential roles of specific cell types in the observed effects}

\section{Dendritic cells}

DCs have been implicated in chapters 2 and 6 to play a role in the oligosaccharideinduced immune modulation, through microbiota-dependent or -independent mechanisms. DCs are known to control T-helper cell polarization (14) and to sample gut content (15). Since they express many PRRs, DCs are potential candidates to play a role in immune-modulatory effects that are caused by the oligosaccharide mixtures. DCs could interact with intestinal bacteria, with the carbohydrate structures of the oligosaccharides themselves or with epithelial cells that are in contact with these factors, resulting in a modulation of DC function. It has been reported that after having sampled intestinal bacteria, intestinal DCs are able to migrate to mucosal sites 
including the MLNs, but not to systemic sites (16). Since the MLNs are sites at which systemic and mucosal immune cells interact, it is possible that the DCs relay immunemodulatory effects in the MLNs over to systemic cells. However, since T cell activation in the vaccination model is expected to have happened at a site distant from the MLNs, it is not clear how the relevant DCs and T-helper cells could have interacted. Possibly, other cells might serve as intermediates between DCs that have interacted with the intestinal microbiota and $\mathrm{T}$ cells that are activated by the vaccination procedure in the periphery.

\section{$\mathrm{CD}_{4}{ }^{+} \mathrm{CD} 25^{+}$regulatory $\mathrm{T}$ cells}

The original concept of Th1 and Th2 cells describes a functional dichotomy in effector T-helper cells (17). Although the concept is still valid, it is recognized nowadays that not all $\mathrm{T}$ cell responses are divided into Th1 or Th2 responses in a black-and-white manner. Moreover, regulatory $\mathrm{T}$ cells exert control over both types of responses and may skew polarization. These cells play a critical role in self-tolerance and the prevention of autoimmune disorders. Many subsets of regulatory $\mathrm{T}$ cell subsets have been described, including the 'natural' thymus-derived $\mathrm{CD} 4{ }^{+} \mathrm{CD} 25^{+} \mathrm{T}$ cells and inducible Th3 and Tr1 CD4+ T cells $(18,19)$.

Various pre- and probiotics have been shown to ameliorate inflammatory conditions such as experimentally induced colitis, in combination with increases in IL-10 and/or TGF- $\beta$; cytokines that are associated with regulatory $\mathrm{T}$ cells (20-22). In addition, inhibition of allergic responses has been observed with multiple probiotic strains (13, $23,24)$. As a result, it has been hypothesized that pre- and probiotics may exert their beneficial effects through modulation of immune-regulatory processes, or, to state it in the context of the hygiene hypothesis, that pre- and probiotics might ameliorate the state of immunological dysregulation that is held responsible for the increased incidences of allergies and autoimmune disorders in industrialized countries (25). This attractive hypothesis automatically leads to the question: could the observed effects of the dietary oligosaccharide mixtures in this thesis be explained by an effect on regulatory cells, specifically, on regulatory $\mathrm{CD} 4{ }^{+} \mathrm{CD} 25^{+} \mathrm{T}$ cells?

$\mathrm{CD} 4{ }^{+} \mathrm{CD} 25^{+} \mathrm{T}$ cells have been shown to play an important role in the OVA allergic asthma model. Transfer of $\mathrm{CD} 4{ }^{+} \mathrm{CD} 25^{+} \mathrm{T}$ cells into OVA sensitized mice reduced symptoms of allergic asthma (26), while inhibition of $\mathrm{CD} 4{ }^{+} \mathrm{CD} 25^{+} \mathrm{T}$ cells by low-dose cyclophosphamide or anti-CD25 antibodies has been shown to exacerbate the allergic condition (27; Schouten and van Esch, personal communication). Therefore, the observed reduction in parameters of allergic asthma in oligosaccharide-supplemented animals (chapter 8 ) could indicate a stimulation of the function of $\mathrm{CD} 4{ }^{+} \mathrm{CD} 25^{+}$cells. 
However, such a mechanism is difficult to reconcile with the observed stimulation of DTH responses in other chapters. Low-dose cyclophosphamide administration prior to vaccination stimulates DTH responses (28), and similar to what was reported in the OVA model, this effect has been recently associated with a decrease in number and function of $\mathrm{CD}^{+}{ }^{+} \mathrm{CD} 25^{+} \mathrm{T}$ cells (29). Moreover, inhibition of DTH responses has been described by stimulating Foxp3-expressing $\mathrm{CD} 4{ }^{+} \mathrm{CD} 25^{+} \mathrm{T}$ cells (30). Thus, oligosaccharide-induced modulation of $\mathrm{CD} 4{ }^{+} \mathrm{CD} 25^{+}$regulatory $\mathrm{T}$ cell function alone cannot consistently explain all observed effects.

\section{Natural killer T cells}

Other cell types than $\mathrm{CD} 4{ }^{+} \mathrm{CD} 25^{+} \mathrm{T}$ cells exert regulatory activities as well. For instance, regulatory $\mathrm{CD}^{+} \mathrm{T}$ cells and NKT cells have also been described play a role in DTH responses (31-33). Regulatory activity of NKT cells has been implicated in many important immunological mechanisms, including self-tolerance, autoimmunity, antitumor responses and microbial interactions (34). These cells express NK receptors and $\alpha \beta$ T cell receptors, either of an invariant (iNKT) or non-invariant type. iNKT cells are known to be CD1d-restricted and can be activated with glycolipids such as $\alpha$ galactosylceramide ( $\alpha$-GalCer) to rapidly produce large amounts of both Th1 and Th2related cytokines.

There are interesting parallels between the results in this thesis and the reports on NKT cell functions. NKT cells do not reach full maturation in C57BL/6 mice before the $10^{\text {th }}$ week of age, judged by their ability to produce IFN- $\gamma$ (35). Blackstock and Murphy demonstrated that adoptive transfer of mature NKT cells at the time of vaccination resulted in enhanced DTH responses in young animals to bacterial antigens at day 7 . In contrast, transfer of premature NKT cells from young mice did not lead to enhanced DTH responses (36). Correspondingly, iNKT cells have been shown to stimulate Th1related immune responses and IFN- $\gamma$ production by others $(37,38)$. In the OVA allergic asthma model, stimulation of iNKT cells with a single dose of $\alpha$-GalCer has been shown to reduce allergic airway inflammation drastically (39-41), although it should be noted that other application schemes were reported to yield the opposite result (42). With regard to antiviral responses, application of $\alpha$-GalCer has been shown to activate NK cell activity in an iNKT-dependent fashion $(43,44)$. Moreover, in vivo administration of $\alpha$-GalCer was shown to reduce early MCMV replication in visceral organs of C57BL/6 mice, as a result of enhanced NK effector function (45). Overall, the whole spectrum of immune-modulatory effects that have been described in this thesis has also been described in association with iNKT activation and function, thereby qualifying these cells as potential intermediates in the immune-modulatory effects of 
prebiotic oligosaccharides, and possibly of probiotic bacteria as well. In correspondence with this idea, an increase in the number of hepatic or splenic NKT cells after a dietary intervention with a prebiotic or probiotic agent is mentioned in two publications (46, 47). In the case of the prebiotic intervention, a stimulation of NK cytotoxicity was observed in spleen cells as well. Unfortunately, the detailed phenotype of the NKT cells was not investigated, making it unclear whether these cells were iNKT or non-invariant NKT cells.

It is unclear how pre- or probiotic agents might affect NKT cells. Although a large number of intra-epithelial NKT cells in mice express non-invariant $\mathrm{T}$ cell receptors, small percentages of iNKT cells have been found in the small and large intestine (reviewed in 48). Not much is known yet about the natural ligands for the invariant $\mathrm{T}$ cell receptors of iNKT cells, but these are thought to comprise glycolipid antigens from Gram-negative bacteria rather than from 'beneficial' Gram-positive bacteria such as bifidobacteria or lactobacilli. In addition, a glycolipid self-antigen may be recognized: isoglobotrihexosylceramide (reviewed in 49). At present, there is no evidence for direct effects of pre- or probiotic agents on (i)NKT cells. However, it is possible that indirect effects might occur through interactions with lamina propria DCs that sample the intestinal content, or with other cells that interact with the intestinal microbiota (50).

It appears that NKT cells have the means and the opportunity to play a key role in the immune-modulatory effects of prebiotic oligosaccharides, but are they guilty? One important point must be taken into account: the literature on iNKT cells is complex and contains many paradoxical reports, while only a small selection was discussed here. Because of this situation, there is a risk that 'just any' effect may be proven by selective use of the literature. Therefore, the proposed hypothesis needs to be corroborated with evidence, before NKT cells can be linked directly to the 'crime'.

\section{Clinical relevance of the observed immune-modulatory effects}

The oligosaccharide concepts that have been tested in this thesis were developed in the first place for application in infant milk formula. It has been reported in literature that breast-feeding reduces the risks of allergy development and the incidence of infections compared to standard infant formula (51-53). In addition, the incidence of allergies is rising in western(ized) countries (54). For these reasons, it is important to develop an improved infant formula for situations in which breast-feeding is not possible, with the goal to reduce the incidence of allergic sensitization and to increase resistance to 
infection. The enhanced DTH responses and reduced early virus replication in supplemented young adult mice is consistent with an increased resistance to infections, whereas the results in the allergic asthma model suggest a reduction in allergic sensitization or symptoms. Overall, these results correspond well with the described goal, but are these results, established in mouse models, relevant for the situation in human infants?

It has been established in chapters 3-5 that the effects of these oligosaccharide mixtures on the murine intestinal microbiota are comparable to the effects in human infants, showing that there are clear similarities between the effects in mice and man. Although the experiments described in this thesis were performed with young adult mice, similar effects of oligosaccharide supplementation have been observed in vaccinated mouse pups, indicating that the effects are relevant in early life (S. Hougee, personal communication). However, the clearest indications of the clinical relevance were shown in a recent clinical study, in which infants received scGOS/lcFOS or placebo-containing formula. It was found that the incidence of atopic dermatitis and other allergy-related parameters were reduced, in combination with a decrease in infectious disease-related parameters, at least for up to one year after the intervention in the test group $(55,56)$. These results show a clear parallel between immunological results in humans and in the mouse models. Since the effects of pAOS in combination with scGOS/lcFOS in the animal experiments were more pronounced than with scGOS/lcFOS alone, it is expected that this will also be the case in humans.

It was argued in chapter 9 that a new, optimal infant formula will likely contain other potentially immune-modulatory ingredients than oligosaccharides, which are to be included for immunological and other reasons. Therefore, the immunologically active ingredients of a novel infant formula concept were tested in a vaccination experiment, to establish whether interaction effects occurred between the ingredients. It was found that low doses of $n-3$ and n-6 PUFA enhanced DTH responses, and surprisingly this appeared to be caused by $n-3$ but not $n-6$ PUFAs in a second experiment. The effect of the combined concept was very similar to the effects of the oligosaccharide mixture alone. Therefore, there is no contra-indication for the application of the combined concept in a novel infant formula, but no additive or synergistic effect was observed either. The rationale for the inclusion of the non-oligosaccharide ingredients is based on other considerations than the results from the vaccination experiments. Therefore, this combined concept will be developed and tested further, with the goal to optimally support the infant's immune function and development.

Aside from applications in infant formula, scGOS, lcFOS and/or pAOS appear promising as well for application in situations of decreased resistance to infection, such 
as in cancer patients or HIV-infected individuals.

\section{Potential effects on autoimmune processes}

Since the discovery of Th1 effector cells in experimental autoimmune models such as experimental autoimmune encephalomyelitis (EAE) and type-I non-obese diabetic (NOD) mice, (uncontrolled) Th1 immune responses have been associated with autoimmunity. At first, it was believed that Th1 responses were detrimental in autoimmune reactions, whereas Th2 responses could ameliorate the condition (57). Not much later, it became clear that this view was too simplistic, as uncontrolled Th2 responses could also lead to autoimmune problems (58). More recently, paradoxical observations with regard to the role of Th1 cytokines in autoimmune disease models (59) have led to a new theory, involving IL-17 producing T-helper cells. These so-called Th17 cells are thought to constitute a new T-helper cell subset apart from Th1, Th2 and regulatory $\mathrm{T}$ cells, which have been suggested to induce pathology in EAE and arthritis models instead of Th1 cells $(60,61)$. As a result, it is not clear that a stimulation of Th1related immune responses will lead to an increased risk of autoimmune disease.

On the other hand, any agent or therapy that modulates immune function may potentially modulate autoimmune processes. Therefore, it is necessary to evaluate the likelihood that application of prebiotic oligosaccharides might lead to increases in the incidence or severity of autoimmune disorders. In the case of probiotic bacteria, concern has been expressed about the safety and the potential effects on autoimmune disorders (62). Opposing results with probiotic intervention have been reported in autoimmune models (63-65), possibly being related to strain-specificity of the effects and/or to differences in the models and experimental procedures. Prebiotic agents differ fundamentally from probiotic bacteria in the sense that no new strains of bacteria are introduced. The modulation of the microbiota is solely induced by providing an altered bacterial substrate, resulting in a selective pressure that favors 'beneficial bacteria', increasing both the amount and the metabolic activity of these bacteria. Still, the question remains whether prebiotics might possibly affect parameters of autoimmunity.

The immunological effects of combinations of scGOS, lcFOS and pAOS were rather subtle compared to pharmacological immune modulators such as $\alpha$-GalCer (39-41). No increases in Th1-related cytokines were observed, and stimulatory effects on DTH responses were only found in Th1-prone C57BL/6 mice, but not in Th2-prone Balb/c mice. Therefore, the chance of inducing autoimmunity is argued to be low. In 
correspondence with this idea, supplementation with $\alpha$-GOS has been reported to ameliorate autoimmune arthritis in rats (66). The same authors also demonstrated that this prebiotic agent could reduce allergic airway eosinophilia (67), resembling the effects in chapter 8 . In contrast, no literature was found in which prebiotic agents were shown to exacerbate autoimmune diseases.

In the case of scGOS/lcFOS, a mixture that is applied already on a wide scale in infant formulas, it was shown that the intestinal microbiota of human infants were modulated in infants that received the oligosaccharides, resulting in a closer resemblance of the microbiota in breast-fed infants (6). The results of a recent trial suggest that application of scGOS/lcFOS in infant formula results in similar anti-allergic and anti-infections effects as have been associated with breast-feeding (51-53), while no adverse effects were observed $(55,56)$. Thus, both in a microbiological and immunological sense, the addition of scGOS/lcFOS to infant formula is suggested to make it more similar to the golden standard, breast milk, and is therefore regarded as safe.

The addition of pAOS to the prebiotic mixture to further mimic the composition of breast milk (the golden standard) enhanced immune-modulatory effects, particularly with regard to DTH responses (chapter 4). Although it is maintained that the risk of increasing the incidence of autoimmune disorders with these oligosaccharides is low as was discussed above, the (immunological) safety of pAOS is currently being assessed with the most appropriate means that are currently available. pAOS has been identified as a 'novel food' by the designated Dutch authority (Dutch Novel Food Committee), thereby requiring careful evaluation and documentation of safety aspects by toxicologists.

Since it is difficult to study effects of (food) components on autoimmune mechanisms in humans, one option is to test pAOS in animal models of autoimmune disease, either as a single component or in combination with scGOS/lcFOS. A potential advantage of such research might be that if the hypothesized involvement of NKT cells is true (68), or if prebiotics could ameliorate a state of immune dysregulation as has been proposed $(25,65)$, parameters of autoimmunity may be inhibited rather than stimulated, thereby providing even more support for application in humans. Because the oligosaccharide mixtures are primarily aimed at applications in infant formula, such an experiment should be focused on potential effects of oligosaccharides on the incidence of autoimmunity, rather than on the severity of a pre-existing autoimmune disorder. A model should be chosen that features spontaneous development of autoimmunity, such as the development of diabetes in NOD mice. In addition to diabetes, these mice have been shown to be prone to a variety of autoimmune disorders. Genetic research has shown that this susceptibility to autoimmune disorders is a complex, multi-factorial 
phenomenon. Immunologically, many important processes have been shown to play a role in determining the disease course, including antigen presentation, $\mathrm{T}$ cell selection, regulatory T cell and NKT cell function (69). This level of complexity and the many levels of regulation that are present make it a suitable model for studying potential effects of (food) components on autoimmune processes. However, as is the case for all animal models, the process of diabetes development in NOD mice is not $100 \%$ identical to the situation in humans (70). Therefore, results in this model should not be interpreted in the first place as a specific model for type-I diabetes, but as a general model for studying the development of autoimmune disorders. The use of experimentally-induced autoimmunity models is discouraged for this specific purpose, as the key process in the development of autoimmunity, namely the breach of selftolerance, is highly manipulated. A vaccination with self-antigens is often applied in induced models, combined with potent adjuvants, making it unclear whether differences in the onset of disease are due to a modulated vaccination response, or to a loss of regulatory activity that normally protects the host from autoimmunity.

The low incidence and late onset (compared to age at which infant formula is consumed) of autoimmune disorders makes it practically impossible to study potential effects of an oligosaccharide-supplemented formula in a prospective clinical trial, since it would require multiple decades of follow-up and an unrealistically high number of study participants. A post-marketing follow-up strategy is considered a highly relevant alternative that will be applied to monitor this issue.

\section{Conclusion and recommendations for future research}

In conclusion, dietary supplementation of mice with combinations of scGOS, lcFOS and pAOS was shown to result in systemic immune modulation. The effects were consistent with a shift in the Th1/Th2 balance towards Th1-related immunity and were accompanied by changes in the microbiota. Both the immunological and microbiological effects were consistently observed in mice and man (see Fig. 1). The reduced allergy- and infection-related parameters that were observed in scGOS/lcFOSreceiving infants indicate that the observed effects in the animal studies are clinically relevant. Moreover, the results suggest that application of the oligosaccharide mixtures in infant formula may help to make it more similar to breast milk from an immunological point of view.

Many questions remain with regard to the underlying working mechanism. Additional research is recommended to establish whether the intestinal microbiota are necessary 


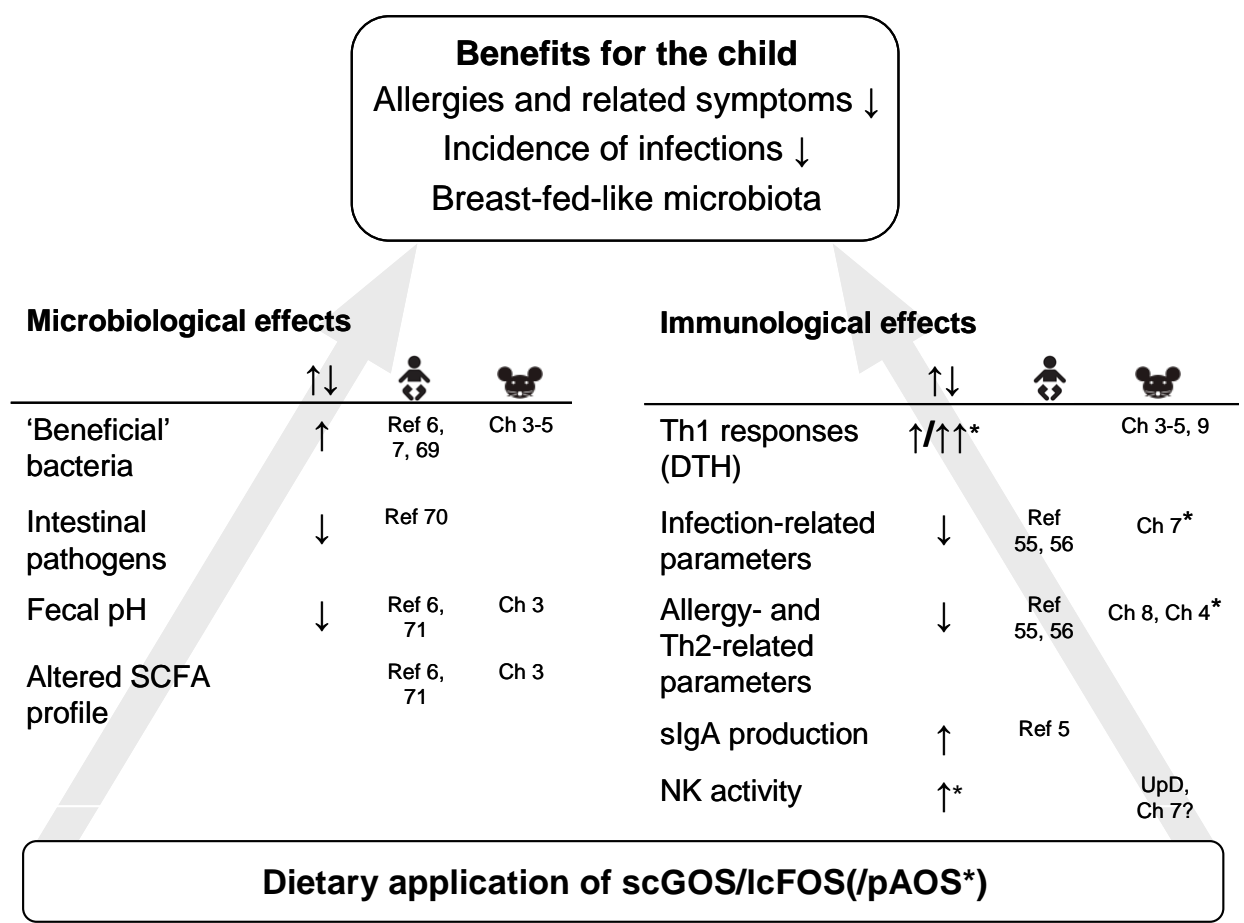

Figure 1. 'Pyramid of evidence' of the most important microbiological and immunological effects in vivo of scGOS/lcFOS(/pAOS). The addition of scGOS/lcFOS to infant formula has been shown to result in various benefits in clinical trials. The most relevant microbiological and immunological effects of dietary scGOS/lcFOS in human infants (literature) and in animal models (this thesis) are listed in the middle section of this schematic overview. These effects are thought to contribute to the benefits that are listed in the top panel, although additional mechanisms might play a role as well. The addition of pAOS to the scGOS/lcFOS mixture has been studied in animal experiments in this thesis; the results that refer only to this extended prebiotic concept are marked with an asterix. $\uparrow$ indicates an upregulation or increase in the parameter, $\downarrow$ a downregulation or decrease. Abbreviations: Ref = literature reference; $C h=$ chapter in this thesis, UpD = unpublished data. The question mark in the reference to chapter 7 denotes the fact that it was hypothesized that NK activity was enhanced, not proven conclusively.

for all immunological effects. For this purpose, germfree mice or systemically administered oligosaccharides could be used to study effects on DTH responses. In addition, more sophisticated in vitro assays could be performed. The involvement of NKT cells may be tested by studying NK1.1+ cell-depleted mice, or by using genetically engineered mice such as CD1d knockout mice. Finally, application of the oligosaccharide mixtures in several human patient groups appears promising. Clinical trials are currently being planned and performed to investigate the effects. 


\section{References}

1.

2.

3.

4.

5.

6.

7.

8.

9.

10.

11.

12.

13.

14.

15.

Tlaskalova-Hogenova, H., L. Tuckova, R. Lodinova-Zadnikova, R. Stepankova, B. Cukrowska, D.P. Funda, I. Striz, H. Kozakova, I. Trebichavsky, D. Sokol, Z. Rehakova, J. Sinkora, P. Fundova, D. Horakova, L. Jelinkova, and D. Sanchez, Mucosal immunity: its role in defense and allergy. Int Arch Allergy Immunol, 2002. 128(2): p. 77-89.

Cross, M.L., Immune-signalling by orally-delivered probiotic bacteria: effects on common mucosal immunoresponses and protection at distal mucosal sites. Int J Immunopathol Pharmacol, 2004. 17(2): p. 127-34.

Hosono, A., A. Ozawa, R. Kato, Y. Ohnishi, Y. Nakanishi, T. Kimura, and R. Nakamura, Dietary fructooligosaccharides induce immunoregulation of intestinal IgA secretion by murine Peyer's patch cells. Biosci Biotechnol Biochem, 2003. 67(4): p. 758-64.

Manhart, N., A. Spittler, H. Bergmeister, M. Mittlbock, and E. Roth, Influence of fructooligosaccharides on Peyer's patch lymphocyte numbers in healthy and endotoxemic mice. Nutrition, 2003. 19(7-8): p. 657-60.

Bakker-Zierikzee, A.M., E.A. Tol, H. Kroes, M.S. Alles, F.J. Kok, and J.G. Bindels, Faecal SIgA secretion in infants fed on pre- or probiotic infant formula. Pediatr Allergy Immunol, 2006. 17(2): p. 134-40.

Knol, J., P. Scholtens, C. Kafka, J. Steenbakkers, S. Gro, K. Helm, M. Klarczyk, H. Schopfer, H.M. Bockler, and J. Wells, Colon microflora in infants fed formula with galacto- and fructo-oligosaccharides: more like breast-fed infants. J Pediatr Gastroenterol Nutr, 2005. 40(1): p. 36-42.

Moro, G.E., F. Mosca, V. Miniello, S. Fanaro, J. Jelinek, B. Stahl, and G. Boehm, Effects of a new mixture of prebiotics on faecal flora and stools in term infants. Acta Paediatr Suppl, 2003. 91(441): p. 77-9.

MacDonald, T.T. and P.B. Carter, Requirement for a bacterial flora before mice generate cells capable of mediating the delayed hypersensitivity reaction to sheep red blood cells. J Immunol, 1979. 122(6): p. 2624-9.

Bartizal, K.F., C. Salkowski, and E. Balish, The influence of a gastrointestinal microflora on natural killer cell activity. J Reticuloendothel Soc, 1983. 33(5): p. 381-9o.

Hori, T., J. Kiyoshima, and H. Yasui, Effect of an oral administration of Lactobacillus casei strain Shirota on the natural killer activity of blood mononuclear cells in aged mice. Biosci Biotechnol Biochem, 2003. 67(2): p. 420-2.

Takagi, A., T. Matsuzaki, M. Sato, K. Nomoto, M. Morotomi, and T. Yokokura, Enhancement of natural killer cytotoxicity delayed murine carcinogenesis by a probiotic microorganism. Carcinogenesis, 2001. 22(4): p. 599-605.

de Waard, R., E. Claassen, G.C. Bokken, B. Buiting, J. Garssen, and J.G. Vos, Enhanced immunological memory responses to Listeria monocytogenes in rodents, as measured by delayed-type hypersensitivity (DTH), adoptive transfer of DTH, and protective immunity, following Lactobacillus casei Shirota ingestion. Clin Diagn Lab Immunol, 2003. 10(1): p. 59-65.

3. Feleszko, W., J. Jaworska, R.D. Rha, S. Steinhausen, A. Avagyan, A. Jaudszus, B. Ahrens, D.A. Groneberg, U. Wahn, and E. Hamelmann, Probiotic-induced suppression of allergic sensitization and airway inflammation is associated with an increase of $T$ regulatory-dependent mechanisms in a murine model of asthma. Clin Exp Allergy, 2007. 37(4): p. 498-505.

de Jong, E.C., H.H. Smits, and M.L. Kapsenberg, Dendritic cell-mediated T cell polarization. Springer Semin Immunopathol, 2005. 26(3): p. 289-307.

Rescigno, M., G. Rotta, B. Valzasina, and P. Ricciardi-Castagnoli, Dendritic cells shuttle microbes across gut epithelial monolayers. Immunobiology, 2001. 204(5): p. 572-81. 
16. Macpherson, A.J. and T. Uhr, Compartmentalization of the mucosal immune responses to commensal intestinal bacteria. Ann N Y Acad Sci, 2004. 1029: p. 36-43.

17. Mosmann, T.R., H. Cherwinski, M.W. Bond, M.A. Giedlin, and R.L. Coffman, Two types of murine helper $T$ cell clone. I. Definition according to profiles of lymphokine activities and secreted proteins. J Immunol, 1986. 136(7): p. 2348-57.

18. Lan, R.Y., A.A. Ansari, Z.X. Lian, and M.E. Gershwin, Regulatory T cells: development, function and role in autoimmunity. Autoimmun Rev, 2005. 4(6): p. 351-63.

19. Cosmi, L., F. Liotta, R. Angeli, B. Mazzinghi, V. Santarlasci, R. Manetti, L. Lasagni, V. Vanini, P. Romagnani, E. Maggi, F. Annunziato, and S. Romagnani, Th2 cells are less susceptible than Th1 cells to the suppressive activity of $C D 25+$ regulatory thymocytes because of their responsiveness to different cytokines. Blood, 2004. 103(8): p. 3117-21.

20. Torii, A., S. Torii, S. Fujiwara, H. Tanaka, N. Inagaki, and H. Nagai, Lactobacillus Acidophilus Strain L92 Regulates the Production of Th1 Cytokine as well as Th2 Cytokines. Allergol Int, 2007. 56(3).

21. Di Giacinto, C., M. Marinaro, M. Sanchez, W. Strober, and M. Boirivant, Probiotics ameliorate recurrent Th1-mediated murine colitis by inducing IL-10 and IL-10-dependent TGF-beta-bearing regulatory cells. J Immunol, 2005. 174(6): p. 3237-46.

22. Hoentjen, F., G.W. Welling, H.J. Harmsen, X. Zhang, J. Snart, G.W. Tannock, K. Lien, T.A. Churchill, M. Lupicki, and L.A. Dieleman, Reduction of colitis by prebiotics in HLA-B27 transgenic rats is associated with microflora changes and immunomodulation. Inflamm Bowel Dis, 2005. 11(11): p. 97785 .

23. Kim, H., S.Y. Lee, and G.E. Ji, Timing of bifidobacterium administration influences the development of allergy to ovalbumin in mice. Biotechnol Lett, 2005. 27(18): p. 1361-7.

24. Forsythe, P., M.D. Inman, and J. Bienenstock, Oral treatment with live Lactobacillus reuteri inhibits the allergic airway response in mice. Am J Respir Crit Care Med, 2007. 175(6): p. 561-9.

25. Guarner, F., R. Bourdet-Sicard, P. Brandtzaeg, H.S. Gill, P. McGuirk, W. van Eden, J. Versalovic, J.V. Weinstock, and G.A. Rook, Mechanisms of disease: the hygiene hypothesis revisited. Nat Clin Pract Gastroenterol Hepatol, 2006. 3(5): p. 275-84.

26. Kearley, J., J.E. Barker, D.S. Robinson, and C.M. Lloyd, Resolution of airway inflammation and hyperreactivity after in vivo transfer of $C D_{4}+C D_{25}+$ regulatory $T$ cells is interleukin 10 dependent. J Exp Med, 2005. 202(11): p. 1539-47.

27. Su, Y.C., M.S. Rolph, M.A. Cooley, and W.A. Sewell, Cyclophosphamide augments inflammation by reducing immunosuppression in a mouse model of allergic airway disease. J Allergy Clin Immunol, 2006. 117(3): p. 635-41.

28. Lagrange, P.H., G.B. Mackaness, and T.E. Miller, Potentiation of T-cell-mediated immunity by selective suppression of antibody formation with cyclophosphamide. J Exp Med, 1974. 139(6): p. 1529-39.

29. Lutsiak, M.E., R.T. Semnani, R. De Pascalis, S.V. Kashmiri, J. Schlom, and H. Sabzevari, Inhibition of $C D_{4}(+) 25^{+} T$ regulatory cell function implicated in enhanced immune response by low-dose cyclophosphamide. Blood, 2005. 105(7): p. 2862-8.

30. Sun, J.B., N. Cuburu, M. Blomquist, B.L. Li, C. Czerkinsky, and J. Holmgren, Sublingual tolerance induction with antigen conjugated to cholera toxin $B$ subunit induces Foxp $3+C D_{2} 5+C D_{4}+$ regulatory $T$ cells and suppresses delayed-type hypersensitivity reactions. Scand J Immunol, 2006. 64(3): p. 251-9.

31. Jiang, H. and L. Chess, An integrated view of suppressor T cell subsets in immunoregulation. J Clin Invest, 2004. 114(9): p. 1198-208.

32. Campos, R.A., M. Szczepanik, A. Itakura, M. Akahira-Azuma, S. Sidobre, M. Kronenberg, and P.W. Askenase, Cutaneous immunization rapidly activates liver invariant Valpha14 NKT cells stimulating $B-1$ $B$ cells to initiate $T$ cell recruitment for elicitation of contact sensitivity. J Exp Med, 2003. 198(12): $p$. $1785-96$. 
33. Szczepanik, M., M. Akahira-Azuma, K. Bryniarski, R.F. Tsuji, I. Kawikova, W. Ptak, C. Kiener, R.A. Campos, and P.W. Askenase, $B-1 B$ cells mediate required early $T$ cell recruitment to elicit proteininduced delayed-type hypersensitivity. J Immunol, 2003. 171(11): p. 6225-35.

34. Delovitch, T.L., The "End of the beginning" of the immunobiology of iNKT cells in health and disease. Int Rev Immunol, 2007. 26(1-2): p. 1-3.

35. Gadue, P. and P.L. Stein, NK T cell precursors exhibit differential cytokine regulation and require Itk for efficient maturation. J Immunol, 2002. 169(5): p. 2397-406.

36. Blackstock, R. and J.W. Murphy, Age-related resistance of $C_{57 B L / 6}$ mice to Cryptococcus neoformans is dependent on maturation of NKT cells. Infect Immun, 2004. 72(9): p. 5175-80.

37. Kawakami, K., Y. Kinjo, S. Yara, K. Uezu, Y. Koguchi, M. Tohyama, M. Azuma, K. Takeda, S. Akira, and A. Saito, Enhanced gamma interferon production through activation of Valpha14(+) natural killer $T$ cells by alpha-galactosylceramide in interleukin-18-deficient mice with systemic cryptococcosis. Infect Immun, 2001. 69(11): p. 6643-50.

38. Kawakami, K., Y. Kinjo, S. Yara, Y. Koguchi, K. Uezu, T. Nakayama, M. Taniguchi, and A. Saito, Activation of Valpha14(+) natural killer $T$ cells by alpha-galactosylceramide results in development of Th1 response and local host resistance in mice infected with Cryptococcus neoformans. Infect Immun, 2001. 69(1): p. 213-20.

39. Morishima, Y., Y. Ishii, T. Kimura, A. Shibuya, K. Shibuya, A.E. Hegab, T. lizuka, T. Kiwamoto, Y. Matsuno, T. Sakamoto, A. Nomura, M. Taniguchi, and K. Sekizawa, Suppression of eosinophilic airway inflammation by treatment with alpha-galactosylceramide. Eur J Immunol, 2005. 35(10): p. 2803-14.

40. Matsuda, H., T. Suda, J. Sato, T. Nagata, Y. Koide, K. Chida, and H. Nakamura, alphaGalactosylceramide, a ligand of natural killer $T$ cells, inhibits allergic airway inflammation. Am J Respir Cell Mol Biol, 2005. 33(1): p. 22-31.

41. Hachem, P., M. Lisbonne, M.L. Michel, S. Diem, S. Roongapinun, J. Lefort, G. Marchal, A. Herbelin, P.W. Askenase, M. Dy, and M.C. Leite-de-Moraes, Alpha-galactosylceramide-induced iNKT cells suppress experimental allergic asthma in sensitized mice: role of IFN-gamma. Eur J Immunol, 2005. 35(10): p. 2793-802.

42. Meyer, E.H., S. Goya, O. Akbari, G.J. Berry, P.B. Savage, M. Kronenberg, T. Nakayama, R.H. DeKruyff, and D.T. Umetsu, Glycolipid activation of invariant T cell receptor+ NK T cells is sufficient to induce airway hyperreactivity independent of conventional $C D_{4}+T$ cells. Proc Natl Acad Sci U S A, 2006. 103(8): p. 2782-7.

43. Wesley, J. and L. Brossay, NK T cell-NK cell cross-talk: reciprocal interaction and activation? Arch Immunol Ther Exp (Warsz), 2003. 51(2): p. 121-6.

44. Ortaldo, J.R., H.A. Young, R.T. Winkler-Pickett, E.W. Bere, Jr., W.J. Murphy, and R.H. Wiltrout, Dissociation of NKT stimulation, cytokine induction, and NK activation in vivo by the use of distinct TCRbinding ceramides. J Immunol, 2004. 172(2): p. 943-53.

45. van Dommelen, S.L., H.A. Tabarias, M.J. Smyth, and M.A. Degli-Esposti, Activation of natural killer (NK) $T$ cells during murine cytomegalovirus infection enhances the antiviral response mediated by $N K$ cells. J Virol, 2003. 77(3): p. 1877-84.

46. Mizubuchi, H., T. Yajima, N. Aoi, T. Tomita, and Y. Yoshikai, Isomalto-oligosaccharides polarize Th1like responses in intestinal and systemic immunity in mice. J Nutr, 2005. 135(12): p. 2857-61.

47. Ohkawara, S., H. Furuya, K. Nagashima, N. Asanuma, and T. Hino, Oral administration of butyrivibrio fibrisolvens, a butyrate-producing bacterium, decreases the formation of aberrant crypt foci in the colon and rectum of mice. J Nutr, 2005. 135(12): p. 2878-83.

48. van Dieren, J.M., C.J. van der Woude, E.J. Kuipers, J.C. Escher, J.N. Samsom, R.S. Blumberg, and E.E. Nieuwenhuis, Roles of CD1d-restricted NKT cells in the intestine. Inflamm Bowel Dis, 2007.

49. Bendelac, A., P.B. Savage, and L. Teyton, The biology of NKT cells. Annu Rev Immunol, 2007. 25: p. 297-336. 
50. Brigl, M., L. Bry, S.C. Kent, J.E. Gumperz, and M.B. Brenner, Mechanism of CD1d-restricted natural killer T cell activation during microbial infection. Nat Immunol, 2003. 4(12): p. 1230-7.

51. van Odijk, J., I. Kull, M.P. Borres, P. Brandtzaeg, U. Edberg, L.A. Hanson, A. Host, M. Kuitunen, S.F. Olsen, S. Skerfving, J. Sundell, and S. Wille, Breastfeeding and allergic disease: a multidisciplinary review of the literature (1966-2001) on the mode of early feeding in infancy and its impact on later atopic manifestations. Allergy, 2003. 58(9): p. 833-43.

52. Gdalevich, M., D. Mimouni, M. David, and M. Mimouni, Breast-feeding and the onset of atopic dermatitis in childhood: a systematic review and meta-analysis of prospective studies. J Am Acad Dermatol, 2001. 45(4): p. 520-7.

53. Cushing, A.H., J.M. Samet, W.E. Lambert, B.J. Skipper, W.C. Hunt, S.A. Young, and L.C. McLaren, Breastfeeding reduces risk of respiratory illness in infants. Am J Epidemiol, 1998. 147(9): p. 863-70.

54. Bjorksten, B., D. Dumitrascu, T. Foucard, N. Khetsuriani, R. Khaitov, M. Leja, G. Lis, J. Pekkanen, A. Priftanji, and M.A. Riikjarv, Prevalence of childhood asthma, rhinitis and eczema in Scandinavia and Eastern Europe. Eur Respir J, 1998. 12(2): p. 432-7.

55. Arslanoglu, S., G. Moro, J. Schmitt, and G. Boehm, Early dietary intervention with a mixture of prebiotic oligosaccharides reduces the incidence of allergy associated symptoms and infections during the first 2 years of life. J Pediatr Gastroenterol Nutr, 2007. 44(S1): p. e217.

56. Moro, G., S. Arslanoglu, B. Stahl, J. Jelinek, U. Wahn, and G. Boehm, A mixture of prebiotic oligosaccharides reduces the incidence of atopic dermatitis during the first six months of age. Arch Dis Child, 2006. 91(10): p. 814-9.

57. Liblau, R.S., S.M. Singer, and H.O. McDevitt, Th1 and Th2 CD4+ T cells in the pathogenesis of organspecific autoimmune diseases. Immunol Today, 1995. 16(1): p. 34-8.

58. Lafaille, J.J., The role of helper $T$ cell subsets in autoimmune diseases. Cytokine Growth Factor Rev, 1998. 9(2): p. 139-51.

59. Gran, B., N. Chu, G.X. Zhang, S. Yu, Y. Li, X.H. Chen, M. Kamoun, and A. Rostami, Early administration of IL-12 suppresses EAE through induction of interferon-gamma. J Neuroimmunol, 2004. 156(1-2): p. 123-31.

6o. Harrington, L.E., R.D. Hatton, P.R. Mangan, H. Turner, T.L. Murphy, K.M. Murphy, and C.T. Weaver, Interleukin 17-producing $C D_{4}+$ effector $T$ cells develop via a lineage distinct from the $T$ helper type 1 and 2 lineages. Nat Immunol, 2005. 6(11): p. 1123-32.

61. Stockinger, B. and M. Veldhoen, Differentiation and function of Th17 T cells. Curr Opin Immunol, 2007. 19(3): p. 281-6.

62. Ezendam, J. and H. van Loveren, Probiotics: immunomodulation and evaluation of safety and efficacy. Nutr Rev, 2006. 64(1): p. 1-14.

63. Calcinaro, F., S. Dionisi, M. Marinaro, P. Candeloro, V. Bonato, S. Marzotti, R.B. Corneli, E. Ferretti, A. Gulino, F. Grasso, C. De Simone, U. Di Mario, A. Falorni, M. Boirivant, and F. Dotta, Oral probiotic administration induces interleukin-1o production and prevents spontaneous autoimmune diabetes in the non-obese diabetic mouse. Diabetologia, 2005. 48(8): p. 1565-75.

64. Baken, K.A., J. Ezendam, E.R. Gremmer, A. de Klerk, J.L. Pennings, B. Matthee, A.A. Peijnenburg, and $\mathrm{H}$. van Loveren, Evaluation of immunomodulation by Lactobacillus casei Shirota: immune function, autoimmunity and gene expression. Int J Food Microbiol, 2006. 112(1): p. 8-18.

65. Matsuzaki, T., Y. Nagata, S. Kado, K. Uchida, I. Kato, S. Hashimoto, and T. Yokokura, Prevention of onset in an insulin-dependent diabetes mellitus model, NOD mice, by oral feeding of Lactobacillus casei. Apmis, 1997. 105(8): p. 643-9.

66. Abe, C., K. Fujita, E. Kikuchi, S. Hirano, H. Kuboki, A. Yamashita, H. Hashimoto, S. Mori, and M. Okada, Effects of alpha-linked galactooligosaccharide on adjuvant-induced arthritis in Wistar rats and type I/ collagen-induced arthritis in DBA/1 J mice. Int J Tissue React, 2004. 26(3-4): p. 65-73. 
67. Sonoyama, K., H. Watanabe, J. Watanabe, N. Yamaguchi, A. Yamashita, H. Hashimoto, E. Kishino, K. Fujita, M. Okada, S. Mori, S. Kitahata, and J. Kawabata, Allergic airway eosinophilia is suppressed in ovalbumin-sensitized Brown Norway rats fed raffinose and alpha-linked galactooligosaccharide. J Nutr, 2005. 135(3): p. 538-43.

68. Novak, J., T. Griseri, L. Beaudoin, and A. Lehuen, Regulation of type 1 diabetes by NKT cells. Int Rev Immunol, 2007. 26(1-2): p. 49-72.

69. Aoki, C.A., A.T. Borchers, W.M. Ridgway, C.L. Keen, A.A. Ansari, and M.E. Gershwin, NOD mice and autoimmunity. Autoimmun Rev, 2005. 4(6): p. 373-9.

70. Roep, B.O., Are insights gained from NOD mice sufficient to guide clinical translation? Another inconvenient truth. Ann N Y Acad Sci, 2007. 1103: p. 1-10.

71. Boehm, G., M. Lidestri, P. Casetta, J. Jelinek, F. Negretti, B. Stahl, and A. Marini, Supplementation of a bovine milk formula with an oligosaccharide mixture increases counts of faecal bifidobacteria in preterm infants. Arch Dis Child Fetal Neonatal Ed, 2002. 86(3): p. F178-81.

72. Knol, J., G. Boehm, M. Lidestri, F. Negretti, J. Jelinek, M. Agosti, B. Stahl, A. Marini, and F. Mosca, Increase of faecal bifidobacteria due to dietary oligosaccharides induces a reduction of clinically relevant pathogen germs in the faeces of formula-fed preterm infants. Acta Paediatr Suppl, 2005. 94(449): p. 313.

73. Bakker-Zierikzee, A.M., M.S. Alles, J. Knol, F.J. Kok, J.J. Tolboom, and J.G. Bindels, Effects of infant formula containing a mixture of galacto- and fructo-oligosaccharides or viable Bifidobacterium animalis on the intestinal microflora during the first 4 months of life. Br J Nutr, 2005. 94(5): p. 783-90. 


\section{Summary}

Pre- and probiotics receive much attention nowadays with regard to their healthpromoting and immune-modulatory properties. In literature, the oligosaccharides in breast milk have been described as the first prebiotic agents that newborns encounter in life, due to their stimulative effect on the number of bifidobacteria in the infant's large intestine. Because there are situations in which it is not possible or preferable to breastfeed infants, it is important that there are infant milk formulas that offer the best possible functional alternative. With this goal, an oligosaccharide concept was previously developed (Immunofortis) for application in infant milk formulas. This mixture, consisting of short-chain galactooligosaccharides and long-chain fructooligosaccharides in a 9:1 ratio (scGOS/lcFOS), was shown to have a similar bifidogenic effect on the composition of the microbiota in infants as breast milk.

Not only do the intestinal microbiota play an important role in digestion, they also interact with cells of the immune system. Moreover, correlations have been described in literature between breast feeding and/or the intestinal microbiota on the one hand, and the incidence of infections and atopic reactions on the other hand. For these reasons, the hypothesis was proposed that specific mixtures of oligosaccharides, based on the scGOS/lcFOS mixture, might induce immune-modulatory effects, leading to a reduction in the incidence of infections and atopic reactions.

The specific goal of the work described in this thesis was to investigate the immunemodulatory effects of specific mixtures of oligosaccharides that are suitable for application in infant nutrition. To reach this goal, animal studies and a limited number of in vitro experiments have been carried out, with a focus on functional changes in the immune system and the consistency of these effects in multiple mouse models. The existing literature on immune-modulatory effects of oligosaccharides and other nondigestible carbohydrates (NDC) was reviewed broadly in chapter 2. One of the important findings was that there are indications for direct, microbiota-independent immune-modulatory effects of NDC, in addition to the more widely accepted microbiota-dependent immunological effects. However, the amount of publications that focus on the underlying mechanism of observed immune-modulatory effects is very limited. Therefore, an attempt was made in this thesis to gain more insight into this mechanism by studying correlations between effects on the microbiota and the immune system, as well as by performing a limited number of in vitro experiments. 
In order to study the immunological effects of specific mixtures of oligosaccharides, an influenza vaccination model was set up in C57BL/6 mice (chapter 3). The vaccination response in this model was regarded to be representative of the functional status of the immune system in general. The delayed-type hypersensitivity (DTH) response, an in vivo T-helper1 (Th1) dependent immune respone, was the most important parameter in this model, but additional immunological and microbiological parameters were quantified as well.

Addition of scGOS/lcFOS to the diets, in amounts that are relevant for application in infant nutrition, led to significantly increased DTH responses after vaccination. Other oligosaccharides did not induce this effect, showing a degree of specificity in the immunological effects of dietary oligosaccharides. Increases in the percentages of bifidobacteria and lactobacilli were observed in mice with increased DTH responses, but the inverse relationship was not always found. This suggests that a prebiotic effect alone is not sufficient for the immunological effect (chapter 3).

The results of chapter 4 correspond well with these findings. In addition to the scGOS/lcFOS concept, pectin-derived acidic oligosaccharides (pAOS) were tested in the vaccination model. When pAOS was added as a single component to the diet, a significant dose-dependent increase in DTH response was observed, as well as a decrease in ex-vivo Th2 cytokine production. However, no significant changes were observed on the microbiological parameters in these mice. A combination of pAOS and scGOS/lcFOS led to a large increase in DTH response that suggested a form of synergy, which was accompanied by an increase in bifidobacteria and lactobacilli in the feces. Overall, the results chapters 3 and 4 are consistent with an oligosaccharide-induced shift in the Th1/Th2 balance towards Th1-mediated immunity in C57BL/6 mice. Since Th1 responses are weak in early life, these results are promising for applications in infant nutrition, with the goal to reduce the incidence of allergies and increase resistance to infections.

Additional vaccination experiments are described in chapter 5, in which the relationship between the effects on the microbiota composition and the immune system was studied. In an initial experiment, the timing of scGOS/lcFOS/pAOS supplementation relative to the vaccination schedule was varied. The results indicated that it is necessary to start supplementation before the first vaccination, in order to obtain a significant increase in DTH response to vaccination. However, supplementation during the second half of the vaccination schedule is not necessary for this effect to occur. These results strongly suggest that the oligosaccharide-induced immune-modulatory effect occurs during the priming phase of the immune response. 
Subsequently, a shortened vaccination experiment was performed to study the correlation between microbiological and immunological parameters shortly after priming. Multiple regression analysis revealed a significant correlation of the DTH response with the percentage of lactobacilli in the cecum, even independently from the oligosaccharide supplementation. Yet, this correlation was not strong and the results suggest that other, unanalyzed parameters played a role as well.

A limited set of in vitro experiments is described in chapter 6 . The goal of these experiments was to find evidence for microbiota-independent immune-modulatory effects of the oligosaccharides that had been studied in vivo. No evidence was found for such effects on parameters of human $\mathrm{T}$ cell function, NK-activity or dendritic cell maturation. However, the lack of evidence in this limited set of experiments does not provide definite proof that microbiota-independent effects of these oligosaccharides do not occur.

In addition to the murine vaccination experiments, disease-specific animal models have been used to study the immune-modulatory effects of the specific mixtures of oligosaccharides. The effects of scGOS/lcFOS/pAOS in a murine cytomegalovirus (MCMV) model is described in chapter 7. It was found that the viral load was significantly reduced in multiple organs early after infection in supplemented animals (C57BL/6) compared to controls. Together with the lower incidence of detectable infectious virus in the liver and increasing trends in interferon- $\alpha$ and $-\gamma$, these data suggest that the oligosaccharides stimulate innate immunity and NK-activity, leading to a reduction in early virus replication. No major effects were observed on MCMVspecific DTH responses in C57BL/6 mice. In BALB/c mice, no significant effects were observed on viral parameters or DTH responses.

It is well-known that BALB/c mice are more Th2-prone than C57BL/6 mice. Therefore, the specific mixtures of scGOS/lcFOS and pAOS were tested in an ovalbumin (OVA)induced model of respiratory allergy, to test the effects on a Th2-related immune response. Supplementation with both scGOS/lcFOS and scGOS/lcFOS/pAOS resulted in significant decreases of respiratory allergy-related parameters, which appeared to be more pronounced in the latter supplementation group. These results show that the immunological effects of specific oligosaccharide mixtures in BALB/c mice are consistent with a shift in the Th1/Th2 balance towards Th1-related immunity, leading to a decrease in the magnitude of Th2 responses.

In chapter 9 a novel concept for infant nutrition, consisting of multiple potentially immune-modulatory ingredients, was tested in the influenza vaccination model. Poly- 
unsaturated fatty acids (PUFA) were part of this concept, because of the beneficial effects on the development of allergies and other parameters that have been described in literature. However, it has also been reported n-3 PUFAs can suppress Th1-related immune parameters. Therefore, the net effect of the total combination of ingredients was studied, to ascertain that the effects of separate ingredients would not cancel each other out. Unexpectedly, a combination of low doses of n-3 and n-6 PUFAS, as well as $n-3$ PUFAs alone led to a significant increase in the DTH response after vaccination. The effect of supplementation with a specific mixture of oligosaccharides induced a larger increase in DTH response, comparable in magnitude to the effect of the total combination of ingredients. These results show that, similar to the effects of the specific oligosaccharide mixtures, the total concept holds promise for reducing the incidence of allergies and increased resistance to infections in infants.

In conclusion, the results in this thesis show that dietary supplementation with combinations of scGOS, lcFOS and pAOS induces systemic immune-modulatory effects in multiple mouse models. These immunological effects are consistent with a shift in the Th1/Th2 balance towards Th1-related immunity, although the results in the MCMV model suggest a stimulation of NK-activity as well. Many questions regarding the precise mechanism behind the immune-modulatory effects have not been answered in this thesis. Additional research is recommended to investigate the potential role of NKT or other regulatory cells in the observed effects, as well as the biological relevance of microbiota-independent effects on the immune system.

The results from clinical studies with scGOS/lcFOS-supplemented infants, in combination with the results in this thesis, show the consistency of the oligosaccharideinduced immunological and microbiological effects in mice and man. The observed reduction in infection- and atopy-related parameters in scGOS/lcFOS-supplemented infants suggests that the application of these oligosaccharides can contribute in a positive way to making infant nutrition more similar to breast milk from an immunological point of view. Additionally, the immunological effects of specific mixtures of oligosaccharides are promising for application in specific nutritional products for adult target groups, such as patient groups that suffer from the consequences of a reduced resistance to infection. Clinical trials with regard to this topic are currently being planned and executed. 


\section{Samenvatting}

Pre- en probiotica staan tegenwoordig in de belangstelling vanwege de gezondheidsbevorderende en immuunmodulerende eigenschappen die eraan worden toegeschreven. Een belangrijke overeenkomst tussen deze voedingscomponenten is dat ze de samenstelling en/of het metabolisme van de micro-organismen (microbiota) in de darm beïnvloeden. De oligosacchariden in moedermelk zijn in de literatuur aangeduid als de eerste natuurlijke prebiotica die pasgeborenen in hun voeding kunnen tegenkomen, vanwege het stimulerende effect ervan op het aantal bifidobacteriën in de dikke darm. Omdat er situaties voorkomen waarin het niet mogelijk of wenselijk is om borstvoeding te geven aan zuigelingen, is het belangrijk dat er alternatieve zuigelingenvoedingen beschikbaar zijn die functioneel zoveel mogelijk gelijk zijn aan borstvoeding. Hiertoe is voorafgaand aan het werk hier beschreven een oligosaccharidenconcept ontwikkeld (Immunofortis, voorheen aangeduid met de afkorting GOS/FOS) voor toepassing in babyvoeding, waarvan is aangetoond dat het een soortgelijk stimulerend effect heeft op bifidobacteriën in zuigelingen als borstvoeding. Dit concept omvat een mix van korte-keten galacto-oligosacchariden en lange-keten fructo-oligosacchariden in een verhouding van 9:1 (scGOS/lcFOS).

De microbiota in de darm spelen niet alleen een belangrijke rol in de menselijke spijsvertering, maar interacteren ook met cellen van het immuunsysteem, waarvan een groot deel in en rond de darmen gelocaliseerd is. In de literatuur zijn er verbanden beschreven tussen borstvoeding en/of de samenstelling van de microbiota enerzijds en het optreden van infecties en allergische reacties anderzijds. Om deze redenen is de hypothese gevormd dat specifieke mengsels van oligosacchariden, gebaseerd op het scGOS/lcFOS concept, mogelijk immuunmodulerende effecten kunnen hebben die resulteren in een vermindering van het ontstaan van allergische reacties en een verhoging van de weerstand tegen infecties.

Het specifieke doel van het werk in dit proefschrift was het onderzoeken van de immuunmodulerende eigenschappen van specifieke oligosaccharidenmengels die kunnen worden toegepast in zuigelingenvoeding. In dit kader zijn dierstudies en een beperkt aantal in vitro experimenten uitgevoerd. De nadruk lag hierbij op het onderzoeken van functionele veranderingen in het immuunsysteem en op de consistentie van deze effecten in verschillende muismodellen. In hoofdstuk 2 is een brede inventarisatie van de literatuur over immuunmodulerende eigenschappen van verschillende oligosacchariden en andere vezels beschreven. Hieruit is onder meer gebleken dat er niet alleen aanwijzingen zijn voor indirekte immunologische effecten 
die worden geïnduceerd via veranderingen in de microbiota, maar ook voor directe, microbiota-onafhankelijke effecten. Er zijn echter maar weinig studies gepubliceerd waarin het onderliggende werkingsmechanisme specifiek is onderzocht. Daarom is er in dit proefschrift ook aandacht besteed aan het werkingsmechanisme dat ten grondslag ligt aan de immunologische effecten, door de correlatie te bestuderen tussen de effecten op het immuunsysteem en de veranderingen in de microbiota, en door verkennende in vitro experimenten uit te voeren.

Om de immunologische effecten van scGOS/lcFOS en andere oligosaccharidenmengsels te testen is een influenza vaccinatiemodel opgezet in C57BL/6 muizen (hoofdstuk 3). Hierbij is de vaccinatie beschouwd als een model-immuunrespons, die is gebruikt als uitleessysteem voor de algemene status van het immuunsysteem in de muizen. Een belangrijke parameter in dit model is de vertraagd-type overgevoeligheidsreactie (DTH respons) die specifiek is voor het vaccin en in vivo gemeten wordt. Deze DTH respons is een T-helper1 (Th1) gemedieerde immuunreactie, een type afweerreactie die van belang is voor de cellulaire immuniteit, zoals de afweer tegen virussen. Th1 reacties staan in balans met T-helper2 (Th2) responsen, die onder andere een rol spelen bij (het ontstaan van) allergieën. Omdat Th1 responsen vlak na de geboorte nog slecht ontwikkeld zijn, is de Th1/Th2 balans in het vroege leven verschoven richting Th2 reacties. In het vaccinatiemodel zijn er naast DTH responsen en additionele immunologische parameters ook microbiologische parameters gemeten in de muizen, om de relatie tussen deze parameters te onderzoeken.

Toevoeging van het scGOS/lcFOS mengsel aan het muizendieet, in hoeveelheden die relevant zijn voor toepassing in babyvoeding, veroorzaakte een significante verhoging van de DTH respons na vaccinatie. Vergelijkbare doses van andere oligosacchariden vertoonden dit effect niet, waaruit kan worden opgemaakt dat de immunologische effecten van verschillende oligosacchariden een mate van specificiteit vertonen. De toename in DTH respons ging gepaard met een toename van het percentage bifidobacteriën en lactobacillen in de feces. Echter, de omgekeerde relatie bleek niet in alle gevallen meetbaar te zijn. Dit suggereert dat een prebiotisch effect alleen niet voldoende (sufficient) is voor het veroorzaken van een immunologisch effect (hoofdstuk 3).

Ook bevindingen in hoofdstuk 4 zijn hiermee in overeenstemming. In uitbreiding op het scGOS/lcFOS oligosaccharidenconcept zijn er van pectine afgeleide zure oligosacchariden (pAOS) getest in het vaccinatiemodel. Als enkele toevoeging aan het dieet induceerde pAOS een significante dosis-afhankelijke toename in de DTH respons, zonder dat er een significant effect op de microbiologische parameters kon worden 
gemeten. Consistent met een verschuiving in de Th1/Th2 balans werd er in dit experiment naast een verhoogde (Th1-afhankelijke) DTH respons ook een verlaagde $e x$ vivo productie van Th2 cytokines gemeten door vaccin-gestimuleerde miltcellen. Een combinatie van pAOS met scGOS/lcFOS gaf een onverwacht grote verhoging van de DTH respons, hetgeen een vorm van synergie suggereert en samen ging met een verhoging van het percentage bifidobacteriën en lactobacillen in de feces na 6 weken oligosaccharidensuppletie. Samengenomen zijn de resultaten van hoofdstukken 3 en 4 consistent met een verschuiving in de Th1/Th2 balans richting Th1-gemedieerde responsen, veroorzaakt door combinaties van scGOS/lcFOS met of zonder pAOS. Vanwege de slecht ontwikkelde Th1-responsen in het vroege leven zijn deze resultaten veelbelovend voor toepassing in babyvoeding, met het doel om het ontstaan van allergieën en het optreden van infecties te verminderen.

Om de relatie tussen de effecten op de microbiota en het immuunsysteem verder te bestuderen zijn er vaccinatie-experimenten uitgevoerd met het oligosaccharidenmengsel dat het meest effectief bleek in het verhogen van de DTH-respons na vaccinatie: scGOS/lcFOS in combinatie met pAOS (hoofdstuk 5). Allereerst is de timing van de oligosaccharidensuppletie gevarieerd ten opzichte van het vaccinatieschema. De resultaten gaven aan dat suppletie voorafgaand aan de primaire vaccinatie noodzakelijk is voor een significant verhoogde DTH respons aan het einde van het zesweekse vaccinatieschema. Anderzijds is suppletie gedurende de tweede helft van het vaccinatieschema hiervoor niet noodzakelijk gebleken; een sterke aanwijzing voor het optreden van de oligosacchariden-geïnduceerde modulatie tijdens de primingsfase van de immuunrespons. Om zo kort mogelijk na de primingsfase de correlatie tussen de microbiologische en immunologische effecten te kunnen meten, is een verkort vaccinatie-experiment uitgevoerd met hetzelfde oligosaccharidenmengsel. Uit multiple-regressie analyse van de DTH resultaten tegen verschillende microbiologische parameters bleek dat het percentage lactobacillen in het cecum een significante correlatie vertoonde met de DTH-respons, zelfs onafhankelijk van de oligosaccharidensuppletie. Evenwel was de correlatie niet heel sterk en duidden de resultaten tevens op een rol van andere, niet analyseerde parameters.

In hoofdstuk 6 staan verschillende in vitro studies beschreven waarin gezocht is naar microbiota-onafhankelijke effecten van oligosacchariden op cellen van het immuunsysteem. Hiertoe zijn verschillende typen humane immuuncellen direct geïncubeerd met oligosacchariden. In perifeer bloed mononucleaire cellen (een mengsel van voornamelijk monocyten, T, B en natural killer (NK) cellen) zijn geen functionele verschillen gevonden in parameters van $\mathrm{T}$ cel functie en NK-activiteit. Ook zijn er geen 
effecten gevonden op parameters van dendritische cel maturatie. Echter, het feit dat er geen aanwijzingen zijn gevonden vormt vanzelfsprekend geen bewijs voor de totale afwezigheid van microbiota-onafhankelijke immunologische effecten.

Naast de influenza vaccinatie-experimenten zijn de oligosaccharidenmengels ook in ziekte-specifieke diermodellen getest, om de functionele effecten van suppletie op parameters van virusinfecties en allergieën te testen. In hoofdstuk 7 zijn de resultaten beschreven van suppletie met een combinatie van scGOS/lcFOS en pAOS in een murine cytomegalovirus (MCMV) infectiemodel, zowel in C57BL/6 als in BALB/c muizen. In C57BL/6 muizen bleek de virusload kort na infectie in meerdere organen significant lager te zijn in gesuppleerde dieren dan in controledieren. Tevens was het aantal dieren met een meetbare hoeveelheid infectieus virus in de lever op verschillende dagen na infectie verlaagd in de gesuppleerde groep. In deze groep werd ook een trend waargenomen van verhoogde interferon- $\alpha$ en $-\gamma$ concentraties in het serum. Dit zou kunnen duiden op een verhoogde activiteit van het 'innate' immuunsysteem, resulterend in een verhoging van de NK-activiteit en een remming van vroege virusreplicatie. Er zijn geen grote verschillen gevonden in virus-specifieke DTH responsen. In tegenstelling tot de resultaten in C57BL/6 muizen zijn er in BALB/C muizen geen significante effecten van de oligosaccharidensuppletie waargenomen in dit model.

Het is bekend dat er immunologische verschillen bestaan tussen C57BL/6 en BALB/C muizen; de eerstgenoemden zijn goede Th1-responders en de laatstgenoemden zijn betere Th2-responders. Om te onderzoeken of effecten in BALB/c muizen wel consistent zijn met de eerder gevonden verschuiving in de Th1/Th2 balans, zijn de oligosacchariden in het ovalbumine-geïnduceerde allergische astma model in BALB/C muizen getest (hoofdstuk 8), waarin Th2-responsen een grote rol spelen. In dit model induceerden scGOS/lcFOS en scGOS/lcFOS/pAOS een significante remming op verschillende allergie-gerelateerde luchtwegparameters. Ook werd er een trend waargenomen van verlaagde ovalbumine-specifieke immunoglobuline E concentraties in het serum. De resultaten suggereerden dat het allergie-remmende effect van scGOS/lcFOS/pAOS sterker was dan van scGOS/lcFOS, echter, door de opzet van de studies kon deze vergelijking niet direct worden gemaakt. Tezamen tonen deze resultaten aan dat de immunologische effecten van de specifieke oligosaccharidenmengsels in BALB/c muizen consistent zijn met een verschuiving in de Th1/Th2 balans richting Th1, waarbij Th2 responsen worden geremd. 
Als laatste is een nieuw te ontwikkelen babyvoeding-concept getest in het influenza vaccinatiemodel (hoofdstuk 9), bestaande uit meerdere potentieel immuunmodulerende stoffen. Meervoudig onverzadigde vetzuren (PUFA) maakten deel uit van dit concept, waarvan in de literatuur gunstige effecten op de ontwikkeling van allergieën en andere parameters zijn beschreven. Echter, van n-3 ('omega-3') PUFA's is ook gerapporteerd dat ze een onderdrukkende werking kunnen hebben op Th1responsen. Daarom is het netto effect van de totale combinatie van ingrediënten bestudeerd, om te controleren of de afzonderlijke componenten elkaars werking niet (deels) zouden opheffen. Enigszins onverwacht leidde dieetsuppletie met lage doses van een combinatie van $n$ - 3 en $n$ - 6 PUFA's, of met $n-3$ PUFA's alleen tot een significant verhoogde DTH respons na vaccinatie. Het effect van oligosaccharidensuppletie leidde tot een groter effect op de DTH respons, vergelijkbaar met de combinatie van alle ingrediënten tezamen. Deze resultaten tonen aan dat het nieuwe concept veelbelovend is voor de ontwikkeling van een nieuwe babyvoeding, met het specifieke doel om de weerstand tegen infecties te verhogen en het ontstaan van allergieën tegen te gaan.

In totaliteit leiden de resultaten in dit proefschrift tot de conclusie dat dieetsuppletie met combinaties van scGOS, lcFOS en pAOS systemische immuunmodulerende effecten veroorzaakt in meerdere experimentele diermodellen. Deze immunologische effecten zijn consistent met een verschuiving in de Th1/Th2 balans richting Th1responsen, al wijzen de resultaten in het MCMV-model tevens in de richting van een stimulatie in NK-activiteit. Aan het einde van dit proefschrift blijven veel vragen over het precieze werkingsmechanisme achter de immunologische effecten onbeantwoord. Additioneel onderzoek wordt aanbevolen om te onderzoeken of NKT-cellen of andere regulerende cellen hierbij betrokken zijn en of microbiota-onafhankelijke effecten op het immuunsysteem een rol spelen.

De resultaten uit klinische studies met scGOS/lcFOS gesuppleerde zuigelingen, in combinatie met de resultaten uit dit proefschrift, tonen de consistentie aan van oligosacchariden-geïnduceerde effecten in mens en dier, zowel in immunologisch als microbiologisch opzicht. De vermindering in het aantal infectie- en allergiegerelateerde parameters die is waargenomen in scGOS/lcFOS-gesuppleerde zuigelingen suggereert dat toepassing hiervan kan bijdragen aan het maken van babyvoeding die in immunologisch opzicht meer op borstvoeding lijkt. Tevens zijn de immunologische effecten van oligosacchariden veelbelovend voor toepassing in specifieke voedingen voor volwassenen, bijvoorbeeld voor specifieke patiëntgroepen waarin de immuunfunctie ondersteund dient te worden. Klinische studies op dit gebied worden op dit moment gepland en uitgevoerd. 


\section{Dankwoord}

Zo, lieve kijkbuiskinderen, het zit erop! Onder het motto: "soms duurt het even, maar dan heb je ook wat" verzamel ik hier de laatste woorden ter afsluiting van mijn boekje en - met gevoel voor drama - van een persoonlijk tijdperk. Gek. Lang heb ik er naar uitgezien, meer dan eens betwijfeld of het ervan zou komen, en nu is het dan zo ver. Een gevoel van voldoening, een vleugje nostalgie, maar vooral is het nog onwerkelijk dat ik straks het achterhoofdgevoel kwijt zal zijn van 'ja maar, ik moet eigenlijk nog schrijven'.

Gezien het verloop van mijn werkzaamheden bij Numico Research - binnenkort Danone Research - heeft het wat langer dan gemiddeld geduurd voordat mijn proefschrift klaar was. In die tijd is er veel gebeurd, met verschillende hoogte- en dieptepunten, zowel op het werk als in mijn privéleven. Er zijn veel mensen die daarbij een belangrijke rol hebben gespeeld en die ik hiervoor expliciet wil bedanken. Overigens heeft de oplettende lezer wellicht opgemerkt dat ik in de afzonderlijke hoofdstukken geen acknowledgements heb opgenomen; dit is geen blijk van gebrek aan erkenning, maar een keuze om deze bijdragen hier wat persoonlijker te benoemen.

Laat ik beginnen bij mijn collega's, de mensen die in het verleden meer dan eens zijn aangeduid met de illustere naam 'Numicoters'. Gedurende de ruim negen jaar dat ik hier aan het werk ben heb ik de algemene sfeer onder collega's altijd erg gewaardeerd. Op momenten dat het werk tegenzat was de gezelligheid en frisheid van deze relatief jonge groep mensen een belangrijke motivatie om door te gaan.

Aan het begin van mijn aanstelling speelde Yvo Graus een grote rol. Yvo, bedankt voor de goede begeleiding, gezelligheid op de kamer (ik hoop dat er op je huidige werkplek minder irritante vliegen zijn) en het zorgen voor een goede uitgangssituatie, waardoor ik later het werk kon uitvoeren dat in dit boekje beschreven staat.

En toen kwam Laura. Nou ja, je was er al een tijd, maar na het vertrek van Yvo kwam ik onder jouw vleug'len. Ik denk dat we behoorlijk verschillende persoonlijkheden zijn, hetgeen naar mijn mening heel positief heeft uitgewerkt vanwege een mate van complementariteit. Dank voor je enthousiasme, je motivatie in tijden van experimentele tegenspoed, je kwaliteiten om dingen geregeld te krijgen, je geduld wanneer ik weer eens was gaan twijfelen aan de handigheid van een eerder bedachte aanpak, je inbreng in dit proefschrift en bovenal de prettige samenwerking door de jaren heen. Super.

Johan, ook jouw bijdrage aan dit werkje was cruciaal. Je enthousiasme is legendarisch, 
net als je DTH kunsten. De vele dagen dat we samen op het CKP waren voor de oormetingen, je supervisie van mijn werk en de sectie in het algemeen, etc. etc.; het was bijzonder nuttig en aangenaam tegelijk. Geweldig dat je mijn promotor bent!

Verbonden aan Numico noch Danone, maar toch jarenlang op afstand betrokken bij het onderzoek was jij, Henk. Je kritische, onafhankelijke blik op het onderzoek en mijn schrijverijen heb ik als zeer waardevol ervaren. Dank voor je inbreng in mijn publicaties, waarvoor je genoegen nam met een eervolle vermelding in de acknowledgements. Co-auteurschappen zijn voor heel wat minder opgeëist. Hopelijk biedt de erkenning van je rol in dit boekje, als eerste promotor, een mooi tegenwicht.

Onder de collega's aan de Bosrandweg is het natuurlijk al jaren een publiek geheim dat de immunologie sectie de leukste is ;-). Ik heb het er dan ook al tijden naar mijn zin en wil alle past \& present sectiegenoten bedanken voor de goede sfeer, collegialiteit, het af en toe even lekker mopperen en dan weer de schouders eronder zetten, de coole uitjes, te veel om op te noemen. Velen van jullie hebben mij door de jaren heen geweldig geholpen met allerlei werkzaamheden, niet in de laatste plaats op de vele 'prakdagen' waarvoor ik verantwoordelijk ben geweest. Karen, ik heb het niet precies bijgehouden, maar misschien heb jij wel de meeste daarvan meegemaakt. Annemarie, Joyce, Suzan en vele anderen hebben daar ook aan bijgedragen, of die experimenten nu wel of niet in mijn boekje terecht zijn gekomen. Ook al was de planning van die dagen niet het populairste werk, de dagen zelf waren altijd erg gezellig en jolig. Enorm bedankt! En Joyce, jouw enorm precieze manier van manuscripten checken mag ook niet onvermeld blijven. Mooi dat je binnen het oncologiewerk nu ook zelf aan de schrijverij bent.

Selva, al wat langer geleden was jij ook regelmatig bij die sectiedagen en een reuze gezellige kamergenoot. Nog recenter, Johanna, en tegenwoordig Jeroen, jullie hebben beide mijn incidentele uitbarstingen van 'publication-' of 'technology-related anger' aan moeten zien. Hopelijk vonden jullie het desondanks net zo aangenaam als ik; rustig, relaxed, op z'n tijd een brainstormpje of een gezellig babbeltje.

Ik was natuurlijk niet de enige in de sectie die af en toe last had van de boekjes-blues. Sander, ehh.... ik bedoel dr. Sander, niet alleen zijn we een tijdlang flatgenoot geweest, het was ook goed om een lotgenoot te hebben in de promotie-business. Datzelfde geldt voor Anita; hopelijk duurt het niet zo heel lang voor we weer een feestje hebben $(;)$. Bea, bij jou zijn de promotieperikelen al heel wat langer achter de rug, maar de samenwerking met Frank et al. in het Maastrichtse heb je mooi geregeld. Voor het hoofdstuk dat dit heeft opgeleverd ben ik jullie beiden zeer erkentelijk. De samenwerking met de Utrechtse Garssianen was ook dermate prettig dat het hier vermeld dient te worden, waarbij ik met name Betty en Gerard wil bedanken voor alle 
inspanningen en het hoofdstuk dat dat onder andere heeft opgeleverd.

Ik heb een aantal studenten mogen begeleiden in de afgelopen jaren, die zeer welkome bijdragen hebben geleverd aan het lopende onderzoek. In het bijzonder wil ik JanWillem en Bob bedanken voor hun werk dat in dit proefschrift terecht is gekomen!

Hilde, ook al heeft onze samenwerking niet direct met mijn proefschrift te maken, hij is erg plezierig. En zo ben ik nog vele (oud-)sectiegenoten dankbaar voor de vele vormen van samenwerking en ondersteuning, variërend van de mentale vorm tot het kopen van demo-CD's van mijn oude bandje.

Ook buiten de sectie zijn er velen wie ik dank verschuldigd ben. Monique, je analyses van de microbiota waren onmisbaar in dit proefschrift. Amra, jij was er ook vaak bij om samples te verzamelen. Hulde. Bernd and Günther, without your brilliant oligosaccharide concept this thesis would not exist. Thank you very much for the cooperation, and to paraphrase a famous German song: vielen Dank für die Zucker! Rob Verdooren, je statistische adviezen waren waardevol en hebben hun weerslag gevonden in menig hoofdstuk. Ook de mensen op het CKP hebben een essentiële bijdrage geleverd aan dit boekje. Bert, jij hebt daar de grootste rol gespeeld in veel van mijn experimenten. Bedankt voor de goede zorgen voor de muizen en de prettige samenwerking. Ook Rene, Frits, Wilma en de vele andere (ex-)medewerkers die hebben bijgedragen: mijn dank is groot!

Ook in mijn persoonlijke kring zijn er veel mensen die ik wil bedanken. Bastiaan, jij bent daar een mooi bruggetje voor. Mede-Garssiaan, vriend, kringgenoot, Klapper, vader van die toffe koter op wie ik af en toe mag passen en man van oppas-mama Adriëlle, bij wie Noah het erg naar d'r zin heeft. Ik vind het erg leuk dat je ook nog eens paranymf wilt zijn. Sehr cool. Samen met Jan, die al eens eerder een ceremoniële rol heeft gespeeld op een belangrijk hoogtepunt in mijn leven. Jan, ik waardeer je vriendschap en het feit dat je paranymf wilt zijn ten zeerste, maar ditmaal laat je je maar flitsen in je eigen auto;-).

Kringgenoten, met jullie is het niet alleen goed musiceren (de bas heeft altijd gelijk natuurlijk), het is ook heel waardevol om samen bezig te zijn met zaken die verder reiken dan het bestek van de wetenschap. Het is bijzonder om lief en leed, vragen en overtuigingen met elkaar te delen, en ik geloof dan ook dat onze betrokkenheid bij elkaar een gave van God is. Ik kan deze manier van in het leven staan iedereen van harte aanbevelen.

Volleyballen bij de Klappers is ook van harte aan te bevelen. Onze wekelijkse wedstrijd op de maandagavond is al jarenlang een plezierige manier om frustraties op sportief 
verantwoorde wijze op de tegenstander te botvieren. Maar net als bij mij persoonlijk wordt het zo langzamerhand wel eens tijd om te gaan promoveren (ok, inkoppertje).

Naast volleyballen is muziek maken ook een belangrijke uitlaatklep voor mij, en ik heb dan ook zeer goede herinneringen aan de vele podia die we hebben bestormd met Catchfly en de experimentele vaagheden die we produceerden met Sst! Wim, Daniël en Floris, wellicht was dit boekje er niet gekomen zonder jullie. En Wim, hopelijk heb ik binnenkort eindelijk weer eens fatsoenlijk tijd voor nieuwe dingen.

Tijd om te zeilen of andere leuke dingen te doen is er hopelijk binnenkort ook weer eens met een ouwe-trouwe vriend als jij, Rob. We moesten maar weer eens iets doen dat mis kan gaan. Rense en Annemien, ook jullie vriendschap door de jaren en gebeurtenissen heen waardeer ik enorm. Er zijn nog veel meer goede vrienden, dichtbij of veraf, die ik hier graag zou willen noemen, maar ik vrees dat er dan helemaal geen einde meer komt aan dit dankwoord. Bij dezen: ik dank $u$ allen.

Pap en mam, jullie verdienen een bijzonder eervolle vermelding in dit dankwoord. Bedankt voor alles wat jullie voor mij hebben gedaan, voor de vrijheid die ik altijd heb gekregen zodat ik me kon ontwikkelen zoals ik wilde, voor jullie trouw door heel wat pieken en dalen heen en voor het enthousiasme waarmee jullie je rol als opa en oma tegenwoordig vervullen. Hans en Ellen, zonder jullie was ik misschien nooit in het Wageningse terechtgekomen. Het was superleuk om een tijdlang met $z^{\prime} n$ allen in of rond Wageningen te wonen, maar ook nu de afstanden wat groter zijn en met Wilma, Judith, Jonathan en Bert erbij zijn jullie geweldige Geschwister!

Maar de grande finale van dit dankwoord is toch echt bestemd voor mijn eigen twee geweldige vrouwen. Allereerst Geja, jouw liefde was onontbeerlijk gedurende al mijn geploeter. Jouw kook- en bakkunsten speelden daarin natuurlijk ook een significante rol ;-). In vele opzichten is het afmaken van dit boekje ook een prestatie van jou. Je niet aflatende steun was en is geweldig; of ik nou wilde promoveren of dacht mijn pipet aan de wilgen te hangen en gitaren te gaan verkopen, je stond vierkant achter me. En dat niet één keer, maar telkens weer. Dat moet wel echte liefde zijn. Daaruit kan alleen maar iets heel moois komen, en dat is dan ook gebeurd. Dit boekje was dan misschien een hele bevalling, de voldoening achteraf valt in het niet bij het krijgen van zo'n lief en schattig meisje als Noah. Noah, je bent een zonnestraaltje in mijn leven en het mooiste meisje dat ik ken. Veel mensen zeggen dat je zo op je moeder lijkt en ik... ik ben het daar van harte mee eens!

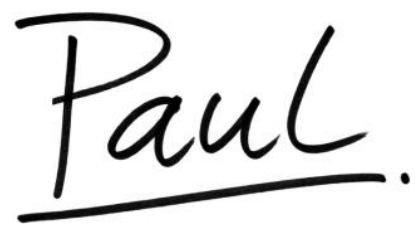




\section{Curriculum Vitae}

Paul Vos was born in IJsselmuiden on 2 February 1975. In 1993, after graduating from the Johannes Calvijn Lyceum in Kampen, he started a study in Biology at the Wageningen University. During his study, he performed two research projects and a traineeship: a project on fish immunology under supervision of Dr. Jan Rombout at the department of Animal Morphology and Cell Biology of the Wageningen University, a project on recombinant human MHC tetramers at the Dutch Cancer Institute in the group of Dr. Ton Schumacher, and a molecular biology traineeship under supervision of Dr. Lorenz Mayr at the Bayer Central Research facility in Leverkusen, Germany.

After completing his study, the author started working at Numico Research* in August 1998, on a project led by Dr. Yvo Graus. Since then, he has worked on various research projects that shared a common direct or indirect aim: to develop nutritional products that provide optimal support for the development and/or function of the immune system in infants, elderly and specific patient groups. The work described in this thesis was performed at Numico Research* under supervision of Dr. ir. Laura M'Rabet and, in part, in collaboration with the Utrecht Institute for Pharmaceutical Sciences and the Maastricht University department of Medical Microbiology.

Currently, the author is employed as a scientist in the Immunology section headed by Prof. dr. Johan Garssen. He is married to Geja Vos-van de Haar and together they have a daughter, Noah (2006).

*Will be called Danone Research - Centre for Specialised Nutrition as of 15 April, 2008. 


\section{List of publications}

M'Rabet, L., A.P. Vos, G. Boehm, and J. Garssen, Breastfeeding and its role on early development of the immune system in infants, consequences for health later in life. J Nutr. Accepted for publication.

Vos, A.P., B.C. van Esch, B. Stahl, L. M'Rabet, G. Folkerts, F.P. Nijkamp, and J. Garssen, Dietary supplementation with specific oligosaccharide mixtures decreases parameters of allergic asthma in mice. Int Immunopharmacol, 2007. 7(12): p. 1582-7.

Vos, A.P., L. M'Rabet, B. Stahl, G. Boehm, and J. Garssen, Immune-modulatory effects and potential working mechanisms of orally applied nondigestible carbohydrates. Crit Rev Immunol, 2007. 27(2): p. 97-140.

Vos, A.P., M. Haarman, J.W. van Ginkel, J. Knol, J. Garssen, B. Stahl, G. Boehm, and L. M'Rabet, Dietary supplementation of neutral and acidic oligosaccharides enhances Th1-dependent vaccination responses in mice. Pediatr Allergy Immunol, 2007. 18(4): p. 304-12.

Vos, A.P., M. Haarman, A. Buco, M. Govers, J. Knol, J. Garssen, B. Stahl, G. Boehm, and L. M'Rabet, A specific prebiotic oligosaccharide mixture stimulates delayed-type hypersensitivity in a murine influenza vaccination model. Int Immunopharmacol, 2006. 6(8): p. 1277-86.

Wouters-Wesseling, W., A.P. Vos, M. Van Hal, L.C. De Groot, W.A. Van Staveren, and J.G. Bindels, The effect of supplementation with an enriched drink on indices of immune function in frail elderly. J Nutr Health Aging, 2005. 9(4): p. 281-6.

Boehm, G., J. Jelinek, J. Knol, L. M'Rabet, B. Stahl, A.P. Vos, and J. Garssen, Prebiotics and immune responses. J Pediatr Gastroenterol Nutr, 2004. 39 Suppl 3: p. S772-3.

Boon, A.C., A.P. Vos, Y.M. Graus, G.F. Rimmelzwaan, and A.D. Osterhaus, In vitro effect of bioactive compounds on influenza virus specific B- and T-cell responses. Scand J Immunol, 2002. 55(1): p. 24-32.

Rombout, J.H., P.H. Joosten, M.Y. Engelsma, A.P. Vos, N. Taverne, and J.J. Taverne-Thiele, Indications for a distinct putative T cell population in mucosal tissue of carp (Cyprinus carpio L.). Dev Comp Immunol, 1998. 22(1): p. 63-77.

\section{Patent applications}

Stahl, B., L. M'Rabet, A.P. Vos, J. Garssen and G. Boehm. Immunemodulating oligosaccharides. WO2005039597.

M'Rabet, L., A. van Baalen, B. Stahl, A.P. Vos and T.H.M. Snoeren, Milk oligosaccharides for stimulating the immune system. WO2007114683. 


\section{Author affiliations}

M. Balvers

G. Boehm

C. Bruggeman

A. Buco

A. Crienen

B.C. van Esch

G. Folkerts

J. Garssen

J.W.H. van Ginkel

M. Govers

G. Grauls

M. Haarman

K. Knipping
Numico Research, Wageningen, The Netherlands

Numico Research, Friedrichsdorf, Germany

Sophia Children's Hospital, Erasmus University, Rotterdam, the Netherlands

Department of Medical Microbiology, Maastricht University, Maastricht, The Netherlands

Numico Research, Wageningen, The Netherlands

Numico Research, Wageningen, The Netherlands

Utrecht Institute of Pharmaceutical Sciences, Utrecht University, Utrecht, The Netherlands

Utrecht Institute of Pharmaceutical Sciences, Utrecht University, Utrecht, The Netherlands

Numico Research, Wageningen, The Netherlands

Utrecht Institute of Pharmaceutical Sciences, Utrecht University, Utrecht, The Netherlands

Numico Research, Wageningen, The Netherlands

Numico Research, Wageningen, The Netherlands

Department of Medical Microbiology, Maastricht University, Maastricht, The Netherlands

Numico Research, Wageningen, The Netherlands

Numico Research, Wageningen, The Netherlands 
J. Knol Numico Research, Wageningen, The Netherlands

B. van 't Land Numico Research, Wageningen, The Netherlands

L. M'Rabet Numico Research, Wageningen, The Netherlands

Utrecht Institute of Pharmaceutical Sciences, Utrecht University, Utrecht, The Netherlands

F.P. Nijkamp Utrecht Institute of Pharmaceutical Sciences, Utrecht University, Utrecht, The Netherlands

B. Sprangers Numico Research, Wageningen, The Netherlands

B. Stahl Numico Research, Friedrichsdorf, Germany

F. Stassen Department of Medical Microbiology, Maastricht University, Maastricht, The Netherlands

Note: As of 15 April, 2008, Numico Research will be called Danone Research - Centre for Specialised Nutrition. 


\section{List of abbreviations}

$\alpha$-GalCer $\quad \alpha$-galactosyl ceramide

AA arachidonic acid

APC antigen presenting cell

AU arbitrary units

BAL broncho-alveolar lavage

CD cluster of differentiation

ConA Concanavalin A

cpm counts per minute

DC dendritic cell (prefix: mo, monocyte-derived)

DHA docosahexaenoic acid

dp degree of polymerization

DSS dextran sodium sulfate

DTH delayed-type hypersensitivity

EPA eicosapentaenoic acid

E:T effector:target

FCS fetal calf serum

FOS fructooligosaccharides (prefixes: sc, short-chain; lc, long-chain; ee, enzymatically elongated)

GLA $\quad \gamma$-linolenic acid

GOS galactooligosaccharides (prefixes: sc, short-chain; $\alpha-/ \beta-$, these indicate the type of sugar linkages between monomer units)

HA haemagglutinin

HMOS human milk oligosaccharides

IBD inflammatory bowel disease

IFN interferon

Ig immunoglobulin

IL interleukin

IMF infant milk formula

iNKT invariant NKT

ip intraperitoneal

LAB lactic acid bacteria

LPS lipopolysaccharide

$\mathrm{mAb}$ monoclonal antibody 
Mch methacholine

MCMV murine cytomegalovirus

MLN mesenteric lymph node

NDC non-digestible carbohydrate

NK natural killer

NKT natural killer T

NOD non-obese diabetic (mice)

OVA ovalbumin

pAOS pectin-derived acidic oligosaccharides

PBMC peripheral blood mononuclear cells

PBS phosphate buffered saline

PENH enhanced pause

PFU plaque-forming unit

PGE2 prostaglandin E2

PMN polymorphonuclear leukocyte

PP Peyer's patch

PRR pattern recognition receptor

PUFA polyunsaturated fatty acid

qPCR quantitave real-time polymerase chain reaction

RS resistant starch

SC subcutaneous

SD standard deviation

SCFA short-chain fatty acids

SEM standard error of the mean

sIgA secretory $\operatorname{Ig} \mathrm{A}$

TGF transforming growth factor

Th1/2 T-helper1/2

TLR Toll-like receptor

TNBS trinitrobenzene sulfonate

TNF tumor necrosis factor 